\title{
TESTING THE ANCIENT MARINE REDOX RECORD FROM OXYGENIC PHOTOSYNTHESIS TO PHOTIC ZONE EUXINA
}

\author{
By \\ Katherine L. French \\ B.S., Yale University, 2009 \\ Submitted in partial fulfillment of the requirements for the degree of \\ Doctor of Philosophy \\ at the
MASSACHUSETTS INSTITUTE OF TECHNOLOGY
and the \\ WOODS HOLE OCEANOGRAPHIC INSTITUTION
}

February 2015

(C) 2015 Katherine L. French

All rights reserved.

The author hereby grants to MIT and WHOI permission to reproduce and distribute publicly paper and electronic copies of this thesis document in whole or in part in any medium now known or hereafter created.

Author:

Joint Program in Chemical Oceanography Massachusetts Institute of Technology and Woods Hole Oceanographic Institution

November 21, 2014

Certified by:

Roger E. Summons

Professor of Geobiology, MIT

Thesis Supervisor

Accepted by:

Elizabeth B. Kujawinski Associate Scientist of Marine Chemistry and Geochemistry, WHOI Chair, Joint Committee for Chemical Oceanography 


\title{
TESTING THE ANCIENT MARINE REDOX RECORD FROM OXYGENIC PHOTOSYNTHESIS TO PHOTIC ZONE EUXINA
}

By

\begin{abstract}
Katherine L. French
Submitted to the Massachusetts Institute of Technology-Woods Hole Oceanographic Institution (MIT/WHOI) Joint Program in Chemical Oceanography on November 21, 2014 in partial fulfillment of the requirements for the degree of Doctor of Philosophy
\end{abstract}

\section{Thesis Abstract}

Tracing the evolution of Earth's redox history is one of the great challenges of geobiology and geochemistry. The accumulation of photosynthetically derived oxygen transformed the redox state of Earth's surface environments, setting the stage for the subsequent evolution of complex life. However, the timing of the advent of oxygenic photosynthesis relative to the Great Oxidation Event (GOE; 2.4 Ga) is poorly constrained. After the deep ocean became oxygenated in the early Phanerozoic, hydrogen sulfide, which is toxic to most aerobes, may have transiently accumulated in the marine photic zone (i.e. photic zone euxinia; PZE) during mass extinctions and oceanic anoxic events. Here, the molecular fossil evidence for oxygenic photosynthesis and eukaryotes is reevaluated, where the results imply that currently existing lipid biomarkers are contaminants. Next, the stratigraphic distribution of green and purple sulfur bacteria biomarkers through geologic time is evaluated to test whether these compounds reflect a water column sulfide signal, which is implicit in their utility as PZE paleoredox proxies. Results from a modern case study underscore the need to consider allochthonous and microbial mat sources and the role of basin restriction as alternative explanations for these biomarkers in the geologic record, in addition to an autochthonous planktonic source.

Thesis supervisor: Roger E. Summons, Professor of Geobiology, MIT 


\section{Acknowledgements}

I would first like to thank my advisor, Roger Summons. Roger has both shaped which research areas I decided to explore and provided the resources that enabled me to answer the questions I found most interesting. Very few students are lucky enough to have an advisor that gives them the freedom to map out their own thesis while also providing the tools to successfully execute the work of their own choosing. For this, his feedback, his assistance, and even teaching me to drive stick shift, I would like to thank Roger. I would also like to thank Carolyn Colonero for her assistance, which has made my work in the Summons lab run as smoothly as possible. More generally, I would like to thank all of the members of the Summons lab that I have overlapped with and even some of the alumni who have become collaborators for the research included in this thesis.

I would also like to thank my committee members, Ann Pearson, Bernhard PeuckerEhrenbrink, and Sam Bowring, for their support and feedback. Their unique perspectives and expertise have been an integral part of this thesis. Thanks to the MIT and WHOI faculty who have taught the courses that have provided the foundation for my graduate research. I am also grateful to the MIT faculty who I have had the opportunity to work with directly, particularly Ed Boyle and Dan Rothman. However, I would also like to thank David McGee, Shuhei Ono, and Tanja Bosak because at some time or another over the past four years I have come to you for assistance and guidance.

I also have to thank my friends who have made my experience in the joint program enjoyable and memorable. In particular, I would like to thank my roommates of 223 (Melissa, Sophie, Becca, Katie, Leticia). I would also like to thank my EAPS and joint program friends. I cannot possibly list all of you, but I hope you know who you are. I would particularly like to thank my office mates, Emily Matys and Rene Boiteau, and all of the folks on the sixth floor of E25. I hope we will have more coffees and teas together in the future. To Deepak, a special thank you. I have learned a lot from all of you while also having a fantastic amount of fun in the process.

Lastly, I cannot say enough to adequately thank my family: my mother, father, brother, and twin sister. I hope I have made the most of the opportunities that my parents have given me through their own hard work and sacrifice. I am so lucky to have a family that is incredibly supportive, fun to spend time with, and who I genuinely count among my best friends.

This research was supported by an NSF graduate research fellowship (DGE-1122374), the NASA Astrobiology Institute (NNA13AA90A), a grant from NASA Exobiology (NNX09AM88G), the Agouron Institute, the Joint Program Academics Program Office, and the PAOC Houghton fund. 


\section{Table of Contents}

$\begin{array}{ll}\text { Chapter 1. Introduction } & 15\end{array}$

Chapter 2. Archean hydrocarbon biomarkers: Syngenetic or not? 27

$\begin{array}{ll}\text { Abstract } & 29\end{array}$

Significance Statement $\quad 30$

2.1 Main Text 30

2.2 Acknowledgements $\quad 40$

2.3 References $\quad 41$

2.4 Supplemental Information $\quad 47$

2.4.1 Drilling 47

2.4.2 Sampling 48

2.4.3 Sawing and Sample Distribution at ANU 49

2.4.4 Laboratory Methodology 52

$\begin{array}{ll}2.4 .5 \text { Results } & 72\end{array}$

2.4.6 Ratio Definitions $\quad 75$

2.4.7 SI References $\quad 76$

Chapter 3. Organic geochemistry of the early Toarcian oceanic anoxic event in Hawsker Bottoms, Yorkshire, England 91

$\begin{array}{ll}\text { Abstract } & 91\end{array}$

3.1 Introduction 94

3.2 Geologic Setting and Site Description of Hawsker Bottoms,

Yorkshire, England $\quad 95$

$\begin{array}{ll}\text { 3.3 Methods } & 97\end{array}$

3.4. Results and Discussion $\quad 98$

3.4.1 Rock-Eval analyses $\quad 98$

3.4.2 Molecular indicators of thermal maturity 99

3.4.3 Biomarker and petrographic evidence of terrigenous

3.4.4 Biomarker indicators of source and community structure $\quad 102$

3.4.5 Indicators of redox change and depositional environment 104

3.4.6 Compound Specific stable carbon isotopic data 110

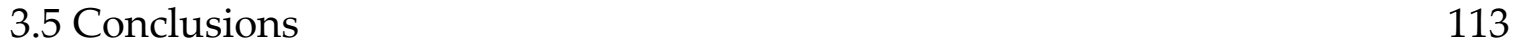

3.6 Acknowledgments $\quad 114$

$\begin{array}{ll}3.7 \text { References } & 115\end{array}$

$\begin{array}{ll}3.8 \text { Supplementary Online Material } & 130\end{array}$ 
Chapter 4. Assessing the distribution of sedimentary $\mathrm{C}_{40}$ carotenoids through time

Abstract

4.1 Introduction

4.2 GSB and PSB carotenoid sources 144

4.3 Materials and Methods $\quad 146$

4.4 Results and Discussion $\quad 147$

4.5 Conclusions 156

4.6 Acknowledgements 157

4.7 References 157

4.8 Supplementary online material $\quad 172$

4.8.1 Analytical conditions for GC-MRM-MS and GC-QQQ-MS $\quad 172$

4.8.2 Construction of stratigraphic distribution plot 173

4.8.3 Supplemental References $\quad 175$

Chapter 5. Evaluating the Benguela upwelling system for anoxygenic photosynthesis as a modern analog of Oceanic Anoxic Events

5.1 Introduction $\quad 184$

5.2 Methods 186

5.3 Results and Discussion $\quad 189$

5.4 Conclusions 193

5.5 Acknowledgements 193

5.6 References 194

Chapter 6. Diagenetic and detrital origin of moretane anomalies through $\begin{array}{ll}\text { the Permian-Triassic boundary } & 209\end{array}$

$\begin{array}{ll}\text { Abstract } & 210\end{array}$

6.1 Introduction $\quad 212$

6.1.1 Moretane Background 213

6.2 Samples and Experimental Methods $\quad 215$

6.2.1 Geographic Setting $\quad 215$

6.2.2 Biomarker Analyses 215

6.2.3 Mineralogical Analyses 216

6.3 Results 218

6.3.1 Stratigraphic Variation of Lithology and Hopane
Distributions

6.3.2 Crossplots of Molecular Indices and Lithological Data 220 
6.3.3 Crossplots of $\mathrm{C}_{35}$ Homohopane Index and Hopane/Sterane ratios with Moretane/Hopane, Ts/(Ts+Tm) and Lithological Data

6.4.1 Possible Causes of Moretane Enrichment

6.4.2 Discussion of Possible Mechanisms for Mineral Preservation of $\beta \alpha$-hopanoid Stereochemistry

6.4.3 Discussion of Depositional Environment, Source Input, and $\mathrm{Ts} /(\mathrm{Ts}+\mathrm{Tm})$

6.4.4 Discussion of Lithology, $\mathrm{C}_{35}$ Homohopane Index and Additional Molecular Parameters

6.4.5 Potential Sources of Berthierine

6.5 Conclusions

232

6.5 Acknowledgements

234

6.7 References 


\section{List of Figures}

\section{Chapter 2}

Fig. 1. Hopanes and Steranes in rock extracts from AIDP-2 compared to RHDH2A.

Fig. 2. Compound Specific and Bulk Organic $\delta^{13} \mathrm{C}$.

Fig. S1. PAH and diamondoid quantification for ANU/MIT Laboratory Analysis.

Fig. S2. Multi-Laboratory Reproducibility of PAH and Diamondoid Ratios.

Fig. S3. HyPy total ion current (TIC) chromatogram of AIDP-2/1/001-116.09m aliphatic hydrocarbons

\section{Chapter 3}

Fig. 1. Rock-Eval Analysis. 123

Fig. 2. Molecular Indicators of Thermal Maturity. 124

Fig. 3. Terrigenous Organic Matter Indicators. 125

Fig. 4. Source and Community Indicators. 126

Fig. 5. Redox and Depositional Environmental Indicators. 127

Fig. 6. Gas chromatography - metastable reaction monitoring mass spectrometry (GC-MRM-MS) chromatogram for the identification of aromatic carotenoid derivatives. 128

$\begin{array}{ll}\text { Fig. 7. Carbon isotopic records. } & 129\end{array}$

\section{Chapter 4}

Fig. 1. Structures of $\mathrm{C}_{40}$ carotenoids 165

Fig. 2. MRM and QQQ chromatograms of aromatic $C_{40}$ carotenoids 166

Fig. 3. Simultaneous detection of aromatic and saturated sedimentary $\mathrm{C}_{40}$ carotenoids by tandem mass spectrometry $\quad 167$

Fig. 4. Stratigraphic distribution of aromatic $\mathrm{C}_{40}$ carotenoids 168

\section{Chapter 5}

Fig. 1. Map of sample stations on Namibian shelf 199

Fig. 2. Response of HPLC-QTOF-MS 200

Fig. 3. FGL sediment extract as an okenone reference 201

Fig. 4. Identification of isorenieratene in FGL sediments 202

Fig. 5. Identification of chlorobactene in FGL sediments 203

Fig. 6. Pigment extracts of Namibian margin sediments 204 
Fig. 7. Bacteriochlorophylls and bacteriopheophytins in reference material

\section{Chapter 6}

Fig. 1. Structures 244

Fig. 2. Multiple reaction monitoring (MRM) chromatograms from sample MS-core1-35-1 at $94.53 \mathrm{~m}$ depth

Fig. 3. Vertical profiles of molecular parameters 246

Fig. 4. Vertical profiles of the bulk lithological components 247

Fig. 5. Vertical profiles of clay types 248

Fig. 6. Cross-correlations of molecular indicies. 249

Fig. 7. Cross-correlations of molecular and bulk lithological data. 250

Fig. 8. Correlations between moretane/hopane ratios and the specific clay types 251

Fig. 9. Correlations of geochemical source and redox indicators against moretane/hopane ratios and $\mathrm{Ts} /(\mathrm{Ts}+\mathrm{Tm})$.

Fig. 10. Correlations of $\mathrm{C}_{35} \mathrm{HHI}(\%)$, a redox indicator, against percent total clay and clay types.

Fig. 11. Correlations of Hopane/Sterane (\%), a geochemical source indicator, against percent total clay and clay types.

\section{Appendix A}

Fig. 1.

Fig. 2.

Fig. 3.

Fig. 4.

Fig. S1.

305

Fig. S2.

305

Fig. S3. 


\section{List of Tables}

\section{Chapter 2}

Table 1. ANU/MIT hopane and sterane concentrations 46

Table S1. ANU/MIT sample description and distribution $\quad 80$

Table S2. ANU/MIT sample preparation $\quad 81$

Table S3. U. Bremen Sample Preparation $\quad 82$

Table S4. Macquarie Sample Preparation 82

Table S5. TOC, bulk organic $\delta^{13} \mathrm{C}$, and $\mathrm{H} / \mathrm{C}$

Table S6. Compound Specific $\delta^{13} \mathrm{C}$ Analysis of extractable PAHs 84

Table S7. Compound Specific $\delta^{13} \mathrm{C}$ Analysis of HyPy products generated from kerogens/extracted rocks 85

Table S8. Extractable PAH \& Diamondoid Ratios 86

Table S9. Calculated Vitrinite Reflectance (\% Rc) 87

Table S10. Selected HyPy yields and ratios of PAH and alkane compounds $\quad 88$

\section{Chapter 3}

Table S1. Figure 1 Data 134

Table S2. Figure 2 Data $\quad 135$

Table S3. Figure 3 Data 136

Table S4. Figure 4 Data 137

Table S5. Figure 5 Data 138

Table S6. Figure 7 Data 139

\section{Chapter 4}

Table 1. MRM and QQQ sample information and $C_{40}$ carotenoid results 169

Table S1. Literature References for Construction of

Figure 3A

\section{Chapter 5}

Table 1. Sample Description 206

Table 2. Diagnostic fragments and identified compounds 207

\section{Chapter 6}

Table 1. Summary of geochemical data 255

Table 2. Bulk lithology 256

Table 3. Absolute and relative clay type percentages 257 


\section{Appendix A}

Table S1.

306

Table S2.

306

Table S3.

306

Table S4.

307

Table S5.

307 


\section{Chapter 1:}

\section{Introduction}

The advent of oxygenic photosynthesis set the stage for the evolution of complex life on an oxygenated planet. It is unknown when this biochemistry emerged, but without question, the oxygenation of the atmosphere and oceans is one of the most profound biologically-mediated transformations of the planet. Moreover, transient marine deoxygenation and sulfide accumulation could have also played a significant role in Phanerozoic mass extinctions and oceanic anoxic events (e.g. 1-3). In the future, it is predicted that oxygen minimum zones (OMZs) and dead zones will expand and intensify, having serious consequences for the biogeochemistry and ecological structure of these marine systems (4-6). Looking into the geologic past and reconstructing Earth's redox history could inform the environmental context during which some metabolisms evolved and expanded in contrast to other intervals where many taxa became rapidly extinct. However, to date, there are many unanswered questions that we must address in order to fully understand Earth's marine redox history and its closely coupled evolutionary drivers and consequences:

1. What is the timing of the advent of oxygenic photosynthesis and the evolution of eukaryotes relative to the Great Oxidation Event (GOE)? 
2. How did the marine redox structure evolve during the Proterozoic? There are few definitive constraints on the nearly two billion year environmental and evolutionary transition between the anoxic Archean oceans and the oxic oceans of the Phanerozoic that we know today.

3. How and why did the marine redox state change during the Phanerozoic oceanic anoxic events (OAEs), and how does oxygen depletion interface with ecological change?

While the following chapters certainly do not solve these three broad questions, they contribute elements of insight to each of these redox-related questions.

The results presented in Chapter 2 have implications for our understanding of when oxygenic photosynthesis and eukaryotes evolved relative to the first major increase in atmospheric oxygen-the GOE. The secular record of mass independent fractionation of sulfur isotopes has become the iconic reflection of the GOE where the mass independent fractionation of ${ }^{33} \mathrm{~S}$ and ${ }^{36} \mathrm{~S}$ relative to ${ }^{32} \mathrm{~S}$ disappears from sedimentary rocks after 2.3-2.4 Ga $(7,8)$, convincingly marking the first step in atmospheric oxygen accumulation $(9,10)$. However, consensus has yet to be reached regarding the antiquity of oxygenic photosynthesis and eukaryotic evolution (10-15). Extracting metabolic and phylogenetic information from the simple morphologies of early microfossils is problematic, so other avenues, particularly geochemical $(14,16-22)$, have been pursued to determine when biological oxygen cycling first evolved. For instance, molecular fossils, particularly hopanes and steranes, detected in Archean rocks have become one of the primary lines of evidence for oxygenesis and aerobiosis as much as 300 million years preceding the $\operatorname{GOE}(14,23-29)$. However, the origin and interpretation of the Archean biomarkers have been questioned in recent years (11, 30- 
32). A multi-laboratory study of new drill cores that tested the syngeneity of hydrocarbon biomarkers reported in Archean host rocks is detailed in Chapter 2.

Molecular and bulk organic geochemical methods are used in Chapter 3 to evaluate the environmental and ecological changes that occurred through the Toarcian OAE. One of the exciting findings reported in this chapter is the first discovery of okenane, which is the diagenetic product of the purple sulfur bacterial carotenoid okenone, in Phanerozoic rocks of marine origin. The diagenetic products of green and purple sulfur bacterial pigments have been used to constrain the depths at which sulfide occured in ancient water columns based on modern observations of the depths where anoxygenic photosynthetic production occurs using specific pigments (33-36). It has been suggested, for instance, that okenane implies sulfide at depths less than $24 \mathrm{~m}$, while isorenieratane implies sulfide at depths as deep as $100 \mathrm{~m}$ (37-39).

Discovering okenane in marine rocks from the Early Jurassic was unexpected because prior to this finding the only marine occurrence of okenane was in the Paleoproterozoic Barney Creek Formation (BCF; 1.64 Ga) (37, 40), which now incidentally contains the oldest hydrocarbon biomarkers according to Chapter 2 . However, unlike the Toarcian event, a stable sulfidic chemocline at depths shallower than $24 \mathrm{~m}$ seems plausible in the Paleoproterozoic because of the prevailing scenario of low atmospheric oxygen concentrations and enhanced anoxygenic photosynthetic production during the Proterozoic (10, 41). In fact, the Barney Creek carotenoids (37, 40 ), in conjunction with abundant inorganic evidence (42-48), have built on and developed the "Canfield Ocean" hypothesis into a more complex narrative of euxinic and ferruginous conditions during protracted deep ocean anoxia of the Proterozoic (49, 50). Yet the Toarcian OAE study in Chapter 3 hinted that the green and purple sulfur bacterial carotenoid record might be incomplete and that there could be additional complexities involved in the intepretation of the carotenoid photic zone euxinia paleoredox proxies. 
Consequently, a more comprehensive study (detailed in Chapter 4) was performed to systematically enhance and assess the patterns of green and purple sulfur bacterial carotenoids in geologic time. As part of this study, two different tandem mass spectrometry analytical methods are described and compared for the analysis of sedimentary $\mathrm{C}_{40}$ carotenoids: gas chromatography metastable reaction monitorgin mass spectrometry (GC-MRM-MS) and gas chromatography triple quadruploe mass spectrometry (GC-QQQ-MS). Together, the results presented in chapters 3 and 4 demonstrate that the green and purple sulfur bacterial carotenoids are more ubiquitous in the geologic record than was previously known. The key question that emerges from this new pattern is how the discovery of okenane in Phanerozoic marine rocks and oils, which were deposited when atmospheric oxygen concentrations were close to modern levels (51), affects the paleoenvironmental interpretation of green and purple sulfur bacterial pigments in samples of all age. More specifically, can a sulfidic chemocline persist at depths of $24 \mathrm{~m}$ or less in an unrestricted marine environment long enough to support green and purple sulfur bacterial production when atmospheric oxygen concentrations are at or near present day levels?

The simpliest approach to answering this question is to look for modern environmental systems where this phenomena occurs. However, one of the challenges that emerges is that there is a "no analog" problem that we face in interpreting the geologic carotenoid record. In other words, at present, there is no evidence in the modern ocean that green and purple sulfur bacteria and their co-occuring pigments are present in unrestricted, transiently sulfidic marine water columns. This is problematic because the green sulfur bacterial carotenoids and inorganic evidence have been used to build a model of expanded transiently euxinic OMZs during Phanerozoic OAEs, rather than the more traditional "ocean stagnation" model $(1,52-56)$. While according to models, this is a more likely model than the "ocean stagnation" model, there is a disconnect between the modern environments that we derive the basis for 
interpretation of green and purple sulfur bacterial carotenoids, such as the Black Sea, fjords, and lakes (e.g. 37-39), and the marine systems that we want to characterize in the ancient: transiently sulfidic OMZs. Yet the modern ocean does supply examples of transient sulfide plumes occuring in OMZs (57-61). What can we learn from them?

The Benguela upwelling system off the coast of Namibia experiences annual sulfide eruptions (61-64). It is not known for how many years the Namibian shelf waters have been prone towards sulfide eruptions, but they have been a feature of the system for more than 50 years (61). Nitrogen cycling has long been the focus of OMZ biogeochemical studies, but recently it was proposed that a "cryptic sulfur cycle" could be operating in today's OMZs $(65,66)$. Documenting sulfide oxidizing photosynthetic bacteria and their pigments in the Benguala upwelling system would add an independent geochemical constraint in support of an active sulfur cycle and would provide a more realistic modern analog of sulfidic OMZs that are envisioned for Phanerozoic OAEs. This prospect is explored further in Chaper 5, where marine sediments of the Namibian margin are surveyed for pigments belonging to green and purple sulfur bacteria.

Another observation that is explored in Chapter 3 is the change in the relative contribution of terrestrial and marine organic matter to the study site in Yorkshire England during the Toarcian OAE. The variability in organic matter sourcing appears to affect the bulk organic matter carbon isotopic excursion (CIE), magnifying it compared to the terrestrial and marine compound specific CIEs. Furthermore, variations in fractional abundance of terrestrial versus marine organic matter have been observed during other OAEs and mass extinctions, where explanations focus on changes in marine productivity, marine organic matter preservation, sea level changes, and terrestrial erosion (e.g. 67-73). In addition to affecting how a CIE is expressed in bulk organic matter, changes in the fractional contribution of terrestrial and marine organic matter to a study site can influence how we characterize marine and terrestrial organic 
carbon cycling during some of these major events. As a result, it is important to identify and accurately interpret the origin for an organic matter source input change. In Chapter 6, several mechanisms, including a change in terrestrial organic matter contribution, are evaluated to explain the sterochemical anomaly that had been previously identified in the $\mathrm{C}_{30}$ hopanes deposited through the Permian-Triassic at the Meishan section of southeastern China. Finally, a new hypothesis for the end Permian carbon cycle perturbation is presented in Appendix 1.

\section{References}

1. Meyer K, Kump L, Ridgwell A (2008) Biogeochemical controls on photic-zone euxinia during the end-Permian mass extinction. Geology 36:747-750.

2. Bambach RK (2006) Phanerozoic biodiversity mass extinctions. Annu Rev Earth Planet Sci 34:127-155.

3. Grice K et al. (2005) Photic zone euxinia during the Permian-Triassic superanoxic event. Science 307:706-709.

4. Diaz RJ, Rosenberg R (2008) Spreading Dead Zones and Consequences for Marine Ecosystems. Science 321:926-929.

5. Stramma L, Johnson GC, Sprintall J, Mohrholz V (2008) Expanding OxygenMinimum Zones in the Tropical Oceans. Science 320:655-658.

6. Stramma L et al. (2011) Expansion of oxygen minimum zones may reduce available habitat for tropical pelagic fishes. Nature Clim Change 2:33-37.

7. Farquhar J, Bao H, Thiemens M (2000) Atmospheric Influence of Earth's Earliest Sulfur Cycle. Science 289:756-758.

8. Bekker A et al. (2004) Dating the rise of atmospheric oxygen. Nature 427:117-120.

9. Kump LR (2008) The rise of atmospheric oxygen. Nature 451:277-278.

10. Lyons TW, Reinhard CT, Planavsky NJ (2014) The rise of oxygen in Earth's early ocean and atmosphere. Nature 506:307-315. 
11. Kirschvink JL, Kopp RE (2008) Palaeoproterozoic ice houses and the evolution of oxygen-mediating enzymes: the case for a late origin of photosystem II. Philosophical Transactions of the Royal Society B: Biological Sciences 363:2755-2765.

12. Kopp R, Kirschvink J, Hilburn I, Nash C (2005) The Paleoproterozoic snowball Earth: a climate disaster triggered by the evolution of oxygenic photosynthesis. Proc Natl Acad Sci USA 102:11131.

13. Rosing MT, Frei R (2004) U-rich Archaean sea-floor sediments from Greenland indications of $>3700$ Ma oxygenic photosynthesis. Earth Planet Sci Lett 217:237-244.

14. Brocks JJ, Logan G, Buick R, Summons RE (1999) Archean molecular fossils and the early rise of eukaryotes. Science 285:1033-1036.

15. Knoll AH, Javaux EJ, Hewitt D, Cohen P (2006) Eukaryotic organisms in Proterozoic oceans. Philosophical Transactions of the Royal Society B: Biological Sciences 361:1023-1038.

16. Garvin J, Buick R, Anbar AD, Arnold GL, Kaufman A (2009) Isotopic evidence for an aerobic nitrogen cycle in the latest Archean. Science 323:1045.

17. Anbar AD et al. (2007) A Whiff of Oxygen Before the Great Oxidation Event? Science 317:1903-1906.

18. Planavsky NJ et al. (2014) Evidence for oxygenic photosynthesis half a billion years before the Great Oxidation Event. Nat Geosci 7:283-286.

19. Kaufman A et al. (2007) Late Archean biospheric oxygenation and atmospheric evolution. Science 317:1900-1903.

20. Kendall B et al. (2010) Pervasive oxygenation along late Archaean ocean margins. Nat Geosci 3:647-652.

21. Scott CT et al. (2011) Late Archean euxinic conditions before the rise of atmospheric oxygen. Geology 39:119-122.

22. Eigenbrode JL, Freeman KH (2006) Late Archean rise of aerobic microbial ecosystems. Proc Natl Acad Sci USA 103:15759-15764.

23. Brocks JJ, Buick R, Summons RE, Logan G (2003) A reconstruction of Archean biological diversity based on molecular fossils from the 2.78 to 2.45 billion-yearold Mount Bruce Supergroup, Hamersley Basin, Western Australia. Geochim Cosmochim Acta 67:4321-4335. 
24. Brocks JJ, Buick R, Logan G, Summons RE (2003) Composition and syngeneity of molecular fossils from the 2.78 to 2.45 billion-year-old Mount Bruce Supergroup, Pilbara Craton, Western Australia. Geochim Cosmochim Acta 67:4289-4319.

25. Waldbauer J, Sherman L, Sumner D, Summons RE (2009) Late Archean molecular fossils from the Transvaal Supergroup record the antiquity of microbial diversity and aerobiosis. Precambrian Res 169:28-47.

26. Eigenbrode JL (2004) Late Archean Microbial Ecology: An Integration of Molecular, Isotopic, and Lithological Studies. PhD Thesis. The Pennsylvania State University, State College, PA. https://etda.libraries.psu.edu/paper/6259/.

27. Eigenbrode JL, Freeman K, Summons RE (2008) Methylhopane biomarker hydrocarbons in Hamersley Province sediments provide evidence for Neoarchean aerobiosis. Earth Planet Sci Lett 273:323-331.

28. George SC, Volk H, Dutkiewicz A, Ridley J, Buick R (2008) Preservation of hydrocarbons and biomarkers in oil trapped inside fluid inclusions for $>2$ billion years. Geochim Cosmochim Acta 72:844-870.

29. Dutkiewicz A, Volk H, George SC, Ridley J, Buick R (2006) Biomarkers from Huronian oil-bearing fluid inclusions: An uncontaminated record of life before the Great Oxidation Event. Geology 34:437-440.

30. Rashby S, Sessions AL, Summons RE, Newman D (2007) Biosynthesis of 2methylbacteriohopanepolyols by an anoxygenic phototroph. Proc Natl Acad Sci USA 104:15099.

31. Rasmussen B, Fletcher I, Brocks JJ, Kilburn M (2008) Reassessing the first appearance of eukaryotes and cyanobacteria. Nature 455:1101-1104.

32. Brocks JJ (2011) Millimeter-scale concentration gradients of hydrocarbons in Archean shales: Live-oil escape or fingerprint of contamination? Geochim Cosmochim Acta 75:3196-3213.

33. Brocks JJ, Summons RE (2003) "Sedimentary Hydrocarbons, biomarkers for early life." In Treatise of Geochemistry (eds. Holland HD, Turekian KK), pp. 63-115.

34. Summons RE, Powell T (1986) Chlorobiaceae in Palaeozoic seas revealed by biological markers, isotopes and geology. Nature 319:763-765.

35. Peters KE, Walters CC, Moldowan JM (2005) The Biomarker Guide: Biomarkers and 
isotopes in petroleum systems and Earth history (Cambridge University Press).

36. Brocks JJ, Grice K (2011) "Biomarkers (molecular fossils)." In Encyclopedia of Geobiology, pp. 147-167. Springer Netherlands.

37. Brocks JJ, Schaeffer P (2008) Okenane, a biomarker for purple sulfur bacteria (Chromatiaceae), and other new carotenoid derivatives from the 1640 Ma Barney Creek Formation. Geochim Cosmochim Acta 72:1396-1414.

38. Repeta DJ, Simpson DJ, Jorgenson BB, Jannasch HW (1989) Evidence for anoxygenic photosynthesis from the distribution of bacterio-chlorophylls in the Black Sea. Nature 342:69-72.

39. Overmann J, Cypionka H, Pfenning N (1992) An extremely low-light-adapted phototrophic sulfur bacterium from the Black Sea. Limnol Oceanogr 37:150-155.

40. Brocks JJ et al. (2005) Biomarker evidence for green and purple sulphur bacteria in a stratified Palaeoproterozoic sea. Nature 437:866-870.

41. Johnston DT, Wolfe-Simon F, Pearson A, Knoll AH (2009) Anoxygenic photosynthesis modulated Proterozoic oxygen and sustained Earth's middle age. Proc Natl Acad Sci USA 106:16925-16929.

42. Shen Y, Canfield DE, Knoll AH (2002) Middle Proterozoic ocean chemistry: Evidence from the McArthur Basin, northern Australia. Am J Sci 302:81-109.

43. Shen Y, Knoll AH, Walter MR (2003) Evidence for low sulphate and anoxia in a mid-Proterozoic marine basin. Nature 423:632-635.

44. Poulton SW, Fralick PW, Canfield DE (2004) The transition to a sulphidic ocean $\sim 1.84$ billion years ago. Nature 431:173-177.

45. Planavsky NJ et al. (2011) Widespread iron-rich conditions in the mid-Proterozoic ocean. Nature 477:448-451.

46. Arnold GL, Anbar AD, Barling J, Lyons T (2004) Molybdenum isotope evidence for widespread anoxia in mid-Proterozoic oceans. Science 304:87-90.

47. Canfield DE et al. (2008) Ferruginous conditions dominated later Neoproterozoic deep-water chemistry. Science 321:949-952.

48. Scott C et al. (2008) Tracing the stepwise oxygenation of the Proterozoic ocean. 
Nature 452:456-459.

49. Lyons TW, Reinhard CT, Scott C (2009) Redox Redux. Geobiol 7:489-494.

50. Canfield DE (1998) A new model for Proterozoic ocean chemistry. Nature 396:450453.

51. Berner RA (2006) GEOCARBSULF: A combined model for Phanerozoic atmospheric $\mathrm{O}_{2}$ and $\mathrm{CO}_{2}$. Geochim Cosmochim Acta 70:5653-5664.

52. Brennecka GA, Herrmann A, Algeo TJ, Anbar AD (2011) Rapid expansion of oceanic anoxia immediately before the end-Permian mass extinction. Proc Natl Acad Sci USA 108:17631-17634.

53. Owens JD et al. (2013) Sulfur isotopes track the global extent and dynamics of euxinia during Cretaceous Oceanic Anoxic Event 2. Proc Natl Acad Sci USA 110:18407-18412.

54. Winguth AME, Maier-Reimer E (2005) Causes of the marine productivity and oxygen changes associated with the Permian-Triassic boundary: A reevaluation with ocean general circulation models. Mar Geol 217:283-304.

55. Erbacher J, Huber BT, Norris RD, Markey M (2001) Increased thermohaline stratification as a possible cause for an ocean anoxic event in the Cretaceous period. Nature 409:325-327.

56. Wilson PA, Norris RD (2001) Warm tropical ocean surface and global anoxia during the mid-Cretaceous period. Nature 412:425-429.

57. Brüchert V, Currie B, Peard KR (2009) Hydrogen sulphide and methane emissions on the central Namibian shelf. Prog Oceanogr 83:169-179.

58. Dugdale RC, Goering J, Barber RT, Smith R, Packard T (1977) Denitrification and hydrogen sulfide in the Peru upwelling region during 1976. Deep-Sea Res 24:601608.

59. Naqvi S et al. (2006) Coastal versus open-ocean denitrification in the Arabian Sea. Biogeosciences 3:621-633.

60. Schunck H et al. (2013) Giant Hydrogen Sulfide Plume in the Oxygen Minimum Zone off Peru Supports Chemolithoautotrophy. PLoS One 8:e68661.

61. Lavik G et al. (2009) Detoxification of sulphidic African shelf waters by blooming 
chemolithotrophs. Nature 457:581-585.

62. Brüchert V et al. (2005) "Biogeochemical and physical control on shelf anoxia and water column hydrogen sulfide in the Benguela coastal upwelling system off Namibia" In Past and Present Water Column Anoxia, ed Neretin LN, pp 161-193.

63. Brüchert V, Currie B, Peard KR (2009) Hydrogen sulphide and methane emissions on the central Namibian shelf. Prog Oceanogr 83:169-179.

64. Emeis KC et al. (2004) Shallow gas in shelf sediments of the Namibian coastal upwelling ecosystem. Cont Shelf Res 24:627-642.

65. Canfield DE et al. (2010) A Cryptic Sulfur Cycle in Oxygen-Minimum-Zone Waters off the Chilean Coast. Science 330:1375-1378.

66. Johnston DT et al. (2014) Placing an upper limit on cryptic marine sulphur cycling. Nature 513:530-533.

67. Kuypers MMM et al. (2004) Orbital forcing of organic carbon burial in the protoNorth Atlantic during oceanic anoxic event 2. Earth Planet Sci Lett 228:465-482.

68. Xie $S$ et al. (2007) Changes in the global carbon cycle occurred as two episodes during the Permian-Triassic crisis. Geology 35:1083-1086.

69. Wang C, Visscher H (2007) Abundance anomalies of aromatic biomarkers in the Permian-Triassic boundary section at Meishan, China--Evidence of end-Permian terrestrial ecosystem collapse. Palaeogeogr Palaeoclimatol Palaeoecol 252:291-303.

70. Wang C (2007) Anomalous hopane distributions at the Permian-Triassic boundary, Meishan, China-Evidence for the end-Permian marine ecosystem collapse. Org Geochem 38:52-66.

71. Nabbefeld B, Grice K, Summons RE, Hays LE, Cao C (2010) Significance of polycyclic aromatic hydrocarbons (PAHs) in Permian/Triassic boundary sections. Appl Geochem 25:1374-1382.

72. Suan G et al. (2011) Polar record of Early Jurassic massive carbon injection. Earth Planet Sci Lett 312:102-113.

73. Pálfy J et al. (2001) Carbon isotope anomaly and other geochemical changes at the Triassic-Jurassic boundary from a marine section in Hungary. Geology 29:10471050. 


\section{Chapter 2:}

\section{Archean hydrocarbon biomarkers: Syngenetic or not?}

This chapter is in review at the Proceedings of the National Academy of Sciences of the United States of America (PNAS) 
Classification: Major: Physical Sciences; Minor: Earth, Atmospheric, and Planetary Sciences; Alternative Major: Biological Sciences; Alternative Minor: Evolution

\section{Archean hydrocarbon biomarkers: Syngenetic or not?}

Authors: Katherine L. French ${ }^{a}$, Christian Hallmann ${ }^{\text {b, c }}$, Janet M. Hoped, Petra L. Schoone, John A. Zumbergee, Yosuke Hoshino ${ }^{\mathrm{f}}$, Carl A. Peters, ${ }^{\mathrm{f}}$, Simon C. George ${ }^{\mathrm{f}}$, Gordon D. Love $^{\mathrm{e}}$, Jochen J. Brocks ${ }^{\mathrm{d}}$, Roger Buickg, Roger E. Summons ${ }^{\mathrm{h}}$

\section{Affiliations:}

aJoint Program in Chemical Oceanography; Massachusetts Institute of Technology and Woods Hole Oceanographic Institution, Cambridge, MA 02139, USA.

bMax Planck Institute for Biogeochemistry, Hans-Knöll-Strasse 10, 07745 Jena, Germany.

cMARUM, University of Bremen, Building IW-3, Am Biologischen Garten 2, 28359 Bremen, Germany.

dResearch School of Earth Sciences, The Australian National University, Canberra ACT 0200, Australia.

eDepartment of Earth Sciences, University of California-Riverside, Riverside, CA 92521, USA.

fDepartment of Earth and Planetary Sciences, Macquarie University, Sydney, NSW 2109, Australia.

sDepartment of Earth \& Space Sciences and Astrobiology Program, University of Washington, Seattle, WA 98195-1310, USA.

hDepartment of Earth, Atmospheric, and Planetary Sciences; Massachusetts Institute of Technology, Cambridge, MA 02139, USA.

${ }^{*}$ Correspondence to: K. L. F

(45 Carleton Street, MIT E25-653; Cambridge, MA 02139; klfrench@mit.edu; +1 $6173243953)$ 
Abstract: Hopanes and steranes found in Archean rocks have been presented as key evidence supporting the early rise of oxygenic photosynthesis and eukaryotes, but the syngeneity of these hydrocarbon biomarkers is controversial. To resolve this debate, we performed a multi-laboratory study of new cores from the Pilbara Craton, Australia that were drilled and sampled using unprecedented hydrocarbon-clean protocols. Hopanes and steranes in rock extracts and hydropyrolysates from these new cores were typically at or below femtogram detection limit, but when they were detectable, they had total hopane ( $<37.9 \mathrm{pg} / \mathrm{g}$ rock) and total sterane ( $<32.9 \mathrm{pg} / \mathrm{g}$ rock) concentrations comparable to those measured in blanks and negative control samples. In contrast, hopanes and steranes measured in the exteriors of conventionally drilled and curated rocks of stratigraphic equivalence reach concentrations as high as $389.5 \mathrm{pg} / \mathrm{g}$ rock and $1039 \mathrm{pg} / \mathrm{g}$ rock, respectively. While polycyclic aromatic hydrocarbons and diamondoids were not detected in laboratory blanks, they exhibit individual concentrations up to $80 \mathrm{ng} / \mathrm{g}$ rock in rock extracts and up to $1000 \mathrm{ng} / \mathrm{g}$ rock in hydropyrolysates from the ultra-clean cores. These new results demonstrate that previously studied Archean samples host mixtures of biomarker contaminants and indigenous thermally overmature hydrocarbons. Therefore, existing lipid biomarker evidence cannot be invoked to support the emergence of oxygenic photosynthesis and eukaryotes by $\sim 2.7 \mathrm{Ga}$. Although suitable Proterozoic rocks exist, no currently known Archean strata lie within the appropriate thermal maturity window for syngenetic lipid biomarker preservation. Future explorations for Archean biomarkers should screen for rocks with milder metamorphic overprints. 
Significance Statement: The advent of oxygenic photosynthesis set the stage for the evolution of complex life on an oxygenated planet, but it is unknown when this transformative biochemistry emerged. The existing lipid biomarker record requires that oxygenic photosynthesis and eukaryotes emerged more than 300 million years before the Great Oxidation Event (GOE). We report that hopane and sterane concentrations measured in new ultra-clean Archean drill cores from Australia are comparable to blank concentrations, yet their concentrations in the exteriors of conventionally collected drill cores of stratigraphic equivalence exceed blank concentrations by more than an order of magnitude due to surficial contamination. Consequently, existing lipid biomarker reports no longer provide valid evidence for the advent of oxygenic photosynthesis and eukaryotes by $\sim 2.7 \mathrm{Ga}$.

Keywords: "Archean biomarkers", "oxygenic photosynthesis", "eukaryotes", "Great Oxidation Event", "Pilbara Craton"

2.1 Main Text: Elucidating the timing of the advent of oxygenic photosynthesis relative to the rise of atmospheric oxygen represents a key challenge in geobiology. The detection of hopane and sterane hydrocarbons in Archean sedimentary rocks has provided an avenue for examining the evolution of biological oxygen cycling (1-7). These results, in addition to inorganic evidence $(8,9)$, suggest that atmospheric oxygen accumulation was suppressed for hundreds of millions of years following the emergence of oxygenic photosynthesis. But the interpretation of the Archean biomarker record has become controversial with recent arguments for a non-syngenetic origin of these hydrocarbons $(10,11)$. Furthermore, the discovery of hopanes and steranes in metamorphosed Archean rocks requires that, if these carbon skeletons are syngenetic, they have persisted despite experiencing temperatures $50-150^{\circ} \mathrm{C}$ above the end of the 
oil window, which is the temperature range over which liquid oil is generated from a source rock $\left(\sim 50-150^{\circ} \mathrm{C}\right)(12)$. In addition to clarifying the upper temperature threshold for lipid biomarker preservation, resolving the origin of the organic biomarkers hosted in Archean rocks would either recast or solidify the late Archean framework in which we understand one of the most profound biologically-mediated transformations of the planet-the Great Oxidation Event (GOE).

We present results from three Archean drill cores from the Pilbara Craton, Western Australia that were drilled during the 2012 Agouron Institute Drilling Program (AIDP). Core AIDP-1 (21 $\left.6^{\prime} 38^{\prime \prime} \mathrm{S}, 119^{\circ} 6^{\prime} 4^{\prime \prime} \mathrm{E}\right)$ intersected the volcanogenic Coucal Formation (3.52 Ga) of the Coonterunah Subgroup (13), while AIDP-2 and AIDP-3 recovered organicrich sedimentary rocks of the lowest metamorphic grade currently known in the Pilbara. Core AIDP-2 was drilled in the Ripon Hills region ( $\left.21^{\circ} 16^{\prime} 51^{\prime \prime S}, 120^{\circ} 50^{\prime} 2 " \mathrm{E}\right)$ as a direct replicate of the RHDH2A core, which was recovered in 1985 using conventional drilling methods (14) and reportedly contains syngenetic Archean biomarkers (3,4). Spatially separated by less than $1 \mathrm{~km}$, AIDP-2 and RHDH2A both intersect the Carawine Dolomite $(<2.63 \mathrm{Ga}$ ) of the Hamersley Group and the Jeerinah Formation (2.63-2.67 Ga) of the Fortescue Group (15), so they are direct equivalents in terms of stratigraphy and regional metamorphism (Fig. 1). Previous studies of mineral assemblages and organic matter reflectivity indicate that the Ripon Hills region lies within the prehnite-pumpellyite metamorphic facies and anthracite coal rank, implying maximum burial temperatures of $200-300^{\circ} \mathrm{C}(16-18)$. AIDP-3 (21 $\left.{ }^{\circ} 46^{\prime} 32^{\prime \prime} \mathrm{S}, 117^{\circ} 34^{\prime} 11^{\prime \prime E}\right)$ was drilled as a deeper water facies analog of AIDP-2 and a stratigraphic equivalent of WRL-1, which is the core that was first reported to contain Archean hydrocarbon biomarkers for cyanobacteria and eukaryotes (1). Like WRL-1, AIDP-3 intersected the Marra Mamba Iron Formation and the Jeerinah Formation and also lies within the prehnite-pumpellyite metamorphic facies (16). 
The AIDP cores were drilled and sampled using protocols chosen to minimize hydrocarbon contamination. At the beginning of the AIDP campaign, all core barrels were cleaned with soap and water, and subsequently only new drill bits were used. Water without added lubricants was used as the sole drilling fluid for the entirety of the program. AIDP-1, which mainly intersected metamorphosed igneous rocks, provided negative control samples (i.e. non-sedimentary rocks devoid of syngenetic organic matter) and abrasively removed residual organics that may have accumulated on the drill-rods during previous projects. Minutes after recovery from depth, untouched portions of selected core material were rinsed with Type-1 ultrapure water in the aluminum core tray before being placed into organic-clean Teflon bags containing several milliliters of ultrapure water to prevent the core material from drying out. After purging with high purity Ar, the sample bags were sealed and frozen at $-20^{\circ} \mathrm{C}$ in the dark until the whole cores were sawed. In total, the whole-core samples were packaged, purged, and frozen in less than 20 minutes after recovery from depth.

Systematic blank analyses using solvent rinses and sawed pieces of a combusted brick demonstrated that the diamond-rimmed blade used with the newly purchased, watercooled saw constructed entirely of stainless steel and aluminum was an important vector for hydrocarbon biomarker contamination. After exhausting other methods (see SI), the saw blade was heated to $300^{\circ} \mathrm{C}$ for 1 hour and was sonicated in solvent, after which solvent rinses and combusted brick blanks verified that sawing no longer contributed detectable hydrocarbon contaminants. Subsequently, the frozen whole core samples were sawed into mirror halves at ANU, which were distributed to multiple laboratories for independent analyses. The extractable biomarker methods and data described below are specific to the ANU/MIT joint dataset, which analyzed the majority 
of samples for extractable hydrocarbons (Table S1), but all results from all laboratories are consistent with the results described here.

Approximately $\sim 5-10 \mathrm{~mm}$ thick slabs of the rock surface were sawed off from the half core, such that all of exposed surfaces were removed (Fig 1). These rock pieces were collected as the "exterior" sample, while the remaining rock served as the "interior" sample. The interior and exterior samples were prepared in parallel with a combusted sand blank. Between $\sim 10-40 \mathrm{~g}$ of the rock powder were extracted with solvents. Each extract was concentrated and redissolved in $100 \mu \mathrm{L}$ of hexane for analyses by gas chromatography-mass spectrometry (GC-MS) in full scan and metastable reaction monitoring (MRM) modes (see SI). Each laboratory analyzed either a chert or igneous negative control sample from AIDP-2 or AIDP-1 for extractable hydrocarbons prior to the analyses of the biomarker target samples. In total, the interiors and exteriors of ten biomarker target samples across the Carawine Dolomite and Jeerinah Formation of AIDP-2 and two biomarker target samples from the Jeerinah Formation of AIDP-3 have been analyzed for extractable hydrocarbons (sample lithologies provided in Table S1).

Hopanes and steranes were typically at or below femtogram detection limits in the rock extracts from AIDP-2 and AIDP-3, but if they were detectable, the total sterane and total hopane concentrations were comparable to blank and negative control concentrations (Table 1; Fig. 1). For comparison, the hopane and sterane concentrations that were detectable and measured in the AIDP samples are $1-3$ orders of magnitude less than the hopane and sterane concentrations reported in earlier Archean biomarker studies (7, 19). Moreover, homologous series of extractable $n$-alkanes $\left(\mathrm{C}_{10-23+}\right)$ that were prominent in previous Archean biomarker studies $(1-3,7)$ were also below detection limits or cumulative laboratory blank concentrations in the analyzed AIDP samples. Hydropyrolysates, which are hydrocarbon pyrolysates released from the kerogen by 
catalytic cracking under high pressure hydrogen, were analyzed from pre-extracted rock powders or kerogens (insoluble organic matter) from 8 samples from AIDP-2 and AIDP-3. As in the rock extracts, individual hopanes and steranes in the AIDP-2 and AIDP-3 hydropyrolysates were usually at or below detection limits, but if they were detectable then neither the total hopane nor the total sterane concentrations exceeded cumulative blank concentrations ( $<20 \mathrm{pg} / \mathrm{g}$ rock). These results are consistent with the absence of hopanes and steranes in previous Archean hydropyrolysis (HyPy) studies $(20,21)$. However, a series of $\mathrm{C}_{10-20} n$-alkanes (with a maximum abundance at $\mathrm{C}_{13}$ ), methylalkanes, and alkylcyclohexanes were detected in hydropyrolysates from two carbonate-rich rocks at $116.09 \mathrm{~m}$ and $170.4 \mathrm{~m}$ in the Carawine Dolomite of AIDP-2. These two samples contain the least thermally altered kerogens according to their association with the highest atomic $\mathrm{H} / \mathrm{C}$ ratios of nearly 0.5 compared to typical values of 0.2 (Table S5) and the highest abundance of alkylated relative to non-alkylated PAH in the pyrolysates (Table S10). The total $n$-alkane concentrations in these hydropyrolysates were two orders of magnitude higher than in laboratory blanks $(<10$ ng/g rock), which did not contain detectable methylalkane and alkylcyclohexane series. The $n$-alkanes detected in the Carawine Formation hydropyrolysate from $116.09 \mathrm{~m}$ in AIDP-2 had $\delta^{13} \mathrm{C}$ values ranging from -38.1 to $-43.6 \%$ (Table S7) compared to the corresponding bulk $\delta^{13} \mathrm{C}$ of $-43.13 \%$ (Table S5). This isotopic similarity indicates that these compounds were generated from the kerogen. In contrast, $n$-alkanes detected in the Jeerinah Formation black shales and more thermally altered Carawine Dolomite hydropyrolysates were comparable to laboratory blank concentrations (Table S10).

To further constrain the source of extractable hydrocarbons previously reported in Archean rocks, a Carawine Dolomite quarter core sample from the RHDH2A core (130.2-130.4 m) was analyzed using the same laboratory protocol described for the AIDP samples. Like the AIDP samples, $25-31 \mathrm{~g}$ of sample interior and exterior were 
extracted (Table S2). The $n$-alkanes, hopanes, and steranes were enriched on the exterior of the RHDH2A core compared to the interior, where the total hopane and total sterane concentrations measured in the exterior exceeded the interior and blank concentrations by 1-2 orders of magnitude (Table 1 and Fig. 1). These patterns substantiate earlier observations of biomarker and bitumen enrichments on the surfaces of conventionally drilled and curated samples relative to the interiors $(3,6,11)$. Furthermore, the hopane and sterane concentrations measured in our analysis of the RHDH2A sample are more comparable to the concentrations reported in earlier Archean biomarker studies of the Pilbara and Kaapvaal cratons $(7,19)$ than the concentrations measured in the AIDP samples. Comparison of RHDH2A and cumulative blank concentrations indicate that laboratory procedures cannot fully account for the addition of biomarkers to RHDH2A. Clearly, the hydrocarbons must have been present in, or added to, the RHDH2A quarter core before laboratory preparation.

Two hypotheses have been proposed to explain surficial biomarker enrichments in Archean drill core: contamination and the live oil effect (i.e. the outward migration of hydrocarbons due to drill core depressurization during recovery) (11). If the live oil effect generated the surficial biomarker enrichments, then samples from both AIDP-2 and RHDH2A should display surficial hopane and sterane enrichments in excess of cumulative blanks. Instead, contamination is the only explanation for the reproducibly detectable surficial hopanes and steranes in conventionally drilled and curated Archean samples $(3,6,7,11)$ and the undetectable or blank level-equivalent hopane and sterane concentrations in samples from the ultra-clean AIDP drill cores.

The spatial distribution of AIDP-2 and AIDP-3 geographically bracket and nearly span the entire stratigraphic section from which all of the other Pilbara biomarkers have been previously reported $(1,3,4,19)$. According to currently available $\mathrm{H} / \mathrm{C}$ ratios and other 
indicators of thermal history, the metamorphic grades of AIDP-2 and AIDP-3 are comparable to $(1-4,6,7,16,19,22)$ or lower than $(5,23,24)$ the metamorphic grades of the rocks from which biomarkers have been previously reported. Moreover, known vectors and signatures of contamination (i.e. surface enrichments) are independent of sampling locality $(6,11$, this study). For example, the analyses of the saw blade (before it was heat-treated) and the sawed surfaces of the RHDH2A quarter core highlight that sawing can contribute significant but variable hydrocarbon contaminants to the substrate, yet the cumulative blanks reported in previous studies did not record the hydrocarbon contribution from sawing $(1,4,6,7)$. Furthermore, recent studies have illustrated that petroleum-derived chemical fossils are prevalent in aerosols, providing another potential vector for hydrocarbon contamination (25). Evidently, samples that were the subject of earlier Archean biomarker work accumulated contaminants on surfaces during processes that were not recorded by laboratory blanks (e.g. drilling, sampling, sawing, and/or storage), and trace amounts of these unaccounted for hydrocarbon contaminants migrated inwards as the samples dried out, oxidized, and fractured (11). Therefore, the results and implications from the new AIDP cores logically extend from the Pilbara Craton to other localities $(1,4-7,19,23,24)$. Since we have not reanalyzed samples from every region using our new rigorous methods, we do not have the equivalent direct evidence that disproves all prior work (e.g. 7). Nevertheless, our new data requires that the null hypothesis should prevail, such that the earlier findings are attributable to contamination based on all currently available evidence. Future studies claiming the discovery of triterpenoid hydrocarbons, even in mildly metamorphosed rocks, must provide overwhelming and robust evidence for syngeneity given that the oil window is the only temperature range over which diagnostic lipid biomarkers are known to survive with certainty at this point (12). 
Many AIDP samples, however, contained extractable polycyclic aromatic hydrocarbons (PAHs) and diamondoids with concentrations up to $80 \mathrm{ng} / \mathrm{g}$ rock and $4 \mathrm{ng} / \mathrm{g}$ rock, respectively (Fig S1). PAHs and diamondoids were also detected in the hydropyrolysates, where individual PAH concentrations were up to $1039 \mathrm{ng} / \mathrm{g}$ rock, and their concentrations were significantly higher than their corresponding blank concentrations of less than $1 \mathrm{ng} / \mathrm{g}$ rock for phenanthrene and pyrene (Table S10). For both rock extracts and hydropyrolysates, the Carawine Dolomite hosted significantly higher concentrations of PAHs and diamondoids than the underlying Jeerinah Formation black shale samples. Like the PAHs and diamondoids that previous studies reported in Archean rock extracts and hydropyrolysates (3, 19, 20, 26), the low molecular weight aromatics $\left(\leq \mathrm{C}_{16}\right)$ predominate over high molecular weight PAHs $(>$ $\left.\mathrm{C}_{16}\right)$, and non-alkylated parent $\mathrm{PAH}$ are in greater abundance compared to alkylated PAH (Fig. S2 and Table S8). Based on extractable PAHs, the MPI-1* parameter yields calculated vitrinite reflectances $\left(R_{c}\right)$ between $2.63-3.00 \%$ (Table S9) $(19,27)$. Although heating rate, duration of heating, and type of organic matter can complicate a direct conversion between $R_{c}$ and temperature, these $R_{c}$ values confirm that these rocks are overmature having experienced temperatures associated with the dry gas window and the lower end of the prehnite-pumpellyite metamorphic temperature range of 200$300^{\circ} \mathrm{C}(16,17,28-30)$. The PAHs and diamondoids are indigenous (i.e. native to the rock prior to drilling) based on several lines of evidence. First, their concentrations in the sample material significantly exceed laboratory blank PAH and diamondoid concentrations. They also have exterior/interior distribution ratios close to one for compounds less sensitive to evaporation. PAHs and diamondoids were detected in both rock extracts and hydropyrolysates. Finally, the PAH and diamondoid distribution and alkylation patterns impart high thermal signatures that match the metamorphic grade. 
Stable carbon isotopic data can be assessed to test whether the indigenous PAHs and diamondoids were generated from the co-existing kerogen (i.e syngenetic) and have the same age as the host rock. Consistent with earlier work $(1,31,32)$, the bulk organic carbon was found to be anomalously ${ }^{13} \mathrm{C}$-depleted with $\delta^{13} \mathrm{C}$ values ranging from -32 to 50\%o (Fig. 2 and Table S5). The extractable PAHs from the Carawine Dolomite were typically ${ }^{13} \mathrm{C}$-enriched by 10 to $20 \%$ o compared to the host rock organic matter (Fig. 2 and Table S6), with two exceptions. First, the sample at $133.55 \mathrm{~m}$ exhibited an isotopic match $(\Delta<0.8 \%$ ) between the extractable PAHs $(-32.3$ to $-33.2 \%$ ) and the associated kerogen $\left(-32.4 \%\right.$ ), which was enriched in ${ }^{13} \mathrm{C}$ by $>10 \%$ o compared to the bulk $\delta^{13} \mathrm{C}$ of the other AIDP samples. This sample, however, is a solid pyrobitumen disk concentrated in a thick stylolitic structure, implying that it could be a migrated petroleum. Notably, the $\delta^{13} \mathrm{C}$ of the PAHs from this unique sample and the PAHs from the other AIDP Carawine Dolomite samples are equivalent, suggesting that they could share the same allochthonous source. Second, three high molecular weight PAHs (228-252 Da) from the AIDP-2 sample at $116.09 \mathrm{~m}$ had isotopic values ranging from -38.9 to $-41.3 \%$, yielding an offset from the host rock kerogen of 1.8 to $4.3 \%$. These three compounds are the most ${ }^{13} \mathrm{C}$-depleted extractable PAHs that have been reported from Archean rocks (1921). Only a single compound specific isotopic measurement was possible for this sample, so the analytical uncertainty and reproducibility are not constrained. In contrast to the extractable PAHs, the $\delta^{13} \mathrm{C}$ of the hydropyrolysate PAHs match the bulk organic matter within a range of 0.1 to $2.9 \%$ o (Fig. 2), and they are the most ${ }^{13} \mathrm{C}$-depleted organic molecules reported from Archean rocks, ranging from -42.6 to $-45.9 \%$ o (Table S7).

Previous work suggests that the Jeerinah Formation and other organic-rich Archean shales generated and expelled petroleum (17) and that the Pilbara craton, including the Ripons Hills region, has experienced multiple episodes of hydrothermal fluid flow, particularly around the time of peak regional metamorphism at $\sim 2.1 \mathrm{Ga}(18,33)$. The 
isotopic match between the AIDP bulk organic matter and the hydropyrolysate PAHs support a syngenetic origin for the hydropyrolysate PAHs where they were generated by thermochemical cleavage of covalent bonds within the host kerogens. In contrast, the $\delta^{13} \mathrm{C}$ of extractable PAHs do not co-vary with the AIDP bulk organic $\delta^{13} \mathrm{C}$ through the carbon isotopic anomaly at 2.6-2.7 Ga (Fig. 2), suggesting that the kerogens of the Jeerinah Formation and Carawine Dolomite may not necessarily be the primary source of the co-occurring extractable PAHs, despite strong evidence of indigeneity. Instead, the primary bitumens may have been diluted or replaced by a second generation of migrated overmature hydrothermal PAHs during or before peak metamorphism. Alternatively, lateral oil migration before peak metamorphism could have introduced the extractable organic matter with isotopic compositions distinct from the associated kerogen. The three extractable PAHs of high molecular weight from the sample at $116.09 \mathrm{~m}$ that have isotopic compositions approaching the bulk organic carbon isotopic composition hint at mixing between syngenetic high molecular weight extractable PAHs and migrated PAHs of low molecular weight containing 1-3 fused aromatic rings, but future studies are required to test this observation. Lastly, the extractable PAHs could be syngenetic but heavily altered by reactions between hydrothermal fluids and the bitumen (26), but this mechanism may not be able to account for the full $>10 \%$ o ${ }^{13} \mathrm{C}$-enrichment. Regardless of the source of the extractable PAHs, the biomolecular precursors of the PAHs and diamondoids are unknown.

We have demonstrated the feasibility of collecting organic-lean and/or overmature drill core samples without the addition of contaminant biomarker hydrocarbons. Our results verify that hydrocarbon concentration gradients through a sample can be used to distinguish contaminants from indigenous hydrocarbons (11), which is a critical proof of concept for Precambrian organic geochemistry that has never been directly demonstrated. Furthermore, HyPy analyses of kerogen-bound organic matter is another 
critical self-consistency check for syngeneity of lipid biomarkers (34). In agreement with previous HyPy studies of Archean rocks (20), hopanes and steranes were not detected in hydropyrolysates from AIDP-2 or AIDP-3. Together, these results from the Pilbara Craton illustrate that previously studied Archean samples host a mixture of indigenous PAHs and diamondoids in addition to hydrocarbon contaminants, including alkanes, steranes, and hopanes that accumulated during sample collection and curation. The Archean sedimentary rocks that are presently known and characterized are too thermally mature to allow for the preservation of syngenetic hopane and sterane molecules, if they had been generated during this time. Future studies aiming to employ molecular organic geochemistry to answer evolutionary questions about the early Earth should screen and filter for samples that have a milder thermal history using a combination of approaches including Rock-Eval pyrolysis, elemental analysis, and Raman spectroscopy. In light of the findings described here, previously reported Archean biomarkers should no longer be invoked as evidence supporting the rise of cyanobacteria and eukaryotes before the GOE.

${ }^{*} \mathrm{MPI}-1=1.5^{*}(2-\mathrm{MeP}+3-\mathrm{MeP}) /(\mathrm{P}+1-\mathrm{MeP}+9-\mathrm{MeP})$ where $P=$ Phenanthrene and $\mathrm{MeP}=$ Methyl Phenanthrene

2.2 Acknowledgments: We thank the Agouron Institute for funding the AIDP drilling and sample analysis and Paul van Loenhout, Lou Lequerica, Jamie Hamilton, and the crew from Mt. Magnet Drilling for their exceptional commitment to drilling cleanliness. We would also like to thank the Yindjibarndi people for granting access to their land, Martin van Kranendonk for organizational and logistical support, Les Bonser for his field support, Kliti Grice for providing assistance and laboratory space that made drilling preparation possible, and John Hayes for constructive comments and 
discussion. We thank the Fallon laboratory at ANU and Ines Hilke and Heike Geilmann at MPI-BCG for assistance with TOC and bulk $\mathrm{d}^{13} \mathrm{C}$ measurements, Mark Williams for assistance with HyPy experiments at UCR, Geoscience Australia, particularly Junhong Chen, for providing CSIA data for the ANU/MIT team, and Enno Schefuß for providing CSIA for C.H. Finally, we acknowledge the Max Planck Society for support provided to C.H., Macquarie University for PhD scholarships awarded to Y.H. and C.A.P, the NSF GRFP for funding support to K.L.F., and the NSF FESD program for additional funding support to R.E.S and G.D.L.

\subsection{References}

1. Brocks JJ, Logan G, Buick R, Summons RE (1999) Archean molecular fossils and the early rise of eukaryotes. Science 285:1033-1036.

2. Brocks JJ, Buick R, Summons RE, Logan G (2003) A reconstruction of Archean biological diversity based on molecular fossils from the 2.78 to 2.45 billion-year-old Mount Bruce Supergroup, Hamersley Basin, Western Australia. Geochim Cosmochim Acta 67:4321-4335.

3. Eigenbrode JL (2004) Late Archean Microbial Ecology: An Integration of Molecular, Isotopic, and Lithological Studies. PhD Thesis. The Pennsylvania State University, State College, PA. https://etda.libraries.psu.edu/paper/6259/.

4. Eigenbrode JL, Freeman KH, Summons RE (2008) Methylhopane biomarker hydrocarbons in Hamersley Province sediments provide evidence for Neoarchean aerobiosis. Earth Planet Sci Lett 273:323-331.

5. Dutkiewicz A, Volk H, George SC, Ridley J, Buick R (2006) Biomarkers from Huronian oilbearing fluid inclusions: An uncontaminated record of life before the Great Oxidation Event. Geology 34:437-440.

6. Sherman LS, Waldbauer JR, Summons RE (2007) Improved methods for isolating and validating indigenous biomarkers in Precambrian rocks. Org Geochem 38:1987-2000.

7. Waldbauer JR, Sherman LS, Sumner DY, Summons RE (2009) Late Archean molecular fossils from the Transvaal Supergroup record the antiquity of microbial diversity and aerobiosis. Precambrian Res 169:28-47. 
8. Anbar AD et al. (2007) A Whiff of Oxygen Before the Great Oxidation Event? Science 317:1903-1906.

9. Lyons TW, Reinhard CT, Planavsky NJ (2014) The rise of oxygen in Earth's early ocean and atmosphere. Nature 506:307-315.

10. Rasmussen B, Fletcher I, Brocks JJ, Kilburn M (2008) Reassessing the first appearance of eukaryotes and cyanobacteria. Nature 455:1101-1104.

11. Brocks JJ (2011) Millimeter-scale concentration gradients of hydrocarbons in Archean shales: Live-oil escape or fingerprint of contamination? Geochim Cosmochim Acta 75:3196-3213.

12. Peters KE, Walters CC, Moldowan JM (2005) The Biomarker Guide: Biomarkers and isotopes in petroleum systems and Earth history (Cambridge University Press).

13. Buick R et al. (1995) Record of emergent continental crust 3.5 billion years ago in the Pilbara craton of Australia. Nature 375:574-577.

14. Richards MN (1985) CRA Exploration Pty. Ltd. Final report on exploration licenses Ripon Hills North 45/63. Ripon Hills South 45/64 and Gingarrigan Creek 45/65, Nullagine, S F 51-5, Western Australia. Item 2655. Western Australia Geological Survey, Report number 13140, WAMEX Item Number 2655, A15932.

15. Rasmussen B, Fletcher IR (2010) Dating sedimentary rocks using in situ U-Pb geochronology of syneruptive zircon in ash-fall tuffs $<1 \mathrm{~mm}$ thick. Geology 38:299-302.

16. Smith RE, Perdrix JL, Parks TC (1982) Burial Metamorphism in the Hamersley Basin, Western Australia. J Petrol 23:75-102.

17. Rasmussen B (2005) Evidence for pervasive petroleum generation and migration in 3.2 and 2.63 Ga shales. Geology 33:497-500.

18. Rasmussen B, Fletcher IR, Sheppard S (2005) Isotopic dating of the migration of a low-grade metamorphic front during orogenesis. Geology 33:773-776.

19. Brocks JJ, Buick R, Logan GA, Summons RE (2003) Composition and syngeneity of molecular fossils from the 2.78 to 2.45 billion-year-old Mount Bruce Supergroup, Pilbara Craton, Western Australia. Geochim Cosmochim Acta 67:4289-4319.

20. Brocks JJ et al. (2003) Release of bound aromatic hydrocarbons from late Archean and Mesoproterozoic kerogens via hydropyrolysis. Geochim Cosmochim Acta 67:1521-1530.

21. Marshall CP et al. (2007) Structural characterization of kerogen in 3.4 Ga Archaean cherts from the Pilbara Craton, Western Australia. Precambrian Res 155:1-23.

22. de Kock MO et al. (2009) Paleomagnetism of a Neoarchean-Paleoproterozoic carbonate ramp 
and carbonate platform succession (Transvaal Supergroup) from surface outcrop and drill core, Griqualand West region, South Africa. Precambrian Res 169:80-99.

23. Ventura GT et al. (2007) Molecular evidence of Late Archean archaea and the presence of a subsurface hydrothermal biosphere. Proc Natl Acad Sci USA 104:14260-14265.

24. George SC, Volk H, Dutkiewicz A, Ridley J, Buick R (2008) Preservation of hydrocarbons and biomarkers in oil trapped inside fluid inclusions for $>2$ billion years. Geochim Cosmochim Acta 72:844-870.

25. Illing CJ, Hallmann C, Miller KE, Summons RE, Strauss H (2014) Airborne hydrocarbon contamination from laboratory atmospheres. Org Geochem 76:26-38.

26. Brocks JJ, Summons RE, Buick R, Logan GA (2003) Origin and significance of aromatic hydrocarbons in giant iron ore deposits of the late Archean Hamersley Basin, Western Australia. Org Geochem 34:1161-1175.

27. Boreham CJ, Crick IH, Powell TG (1988) Alternative calibration of the Methylphenanthrene Index against vitrinite reflectance: Application to maturity measurements on oils and sediments. Org Geochem 12:289-294.

28. Waples DW (1980) Time and Temperature in Petroleum Formation: Application of Lopatin's Method to Petroleum Exploration. AAPG Bulletin 64:916-926.

29. Burnham AK, Sweeney JJ (1989) A chemical kinetic model of vitrinite maturation and reflectance. Geochim Cosmochim Acta 53:2649-2657.

30. Sweeney JJ, Burnham AK (1990) Evaluation of a Simple Model of Vitrinite Reflectance Based on Chemical Kinetics. AAPG Bulletin 74:1559-1570.

31. Eigenbrode JL, Freeman KH (2006) Late Archean rise of aerobic microbial ecosystems. Proc Natl Acad Sci USA 103:15759-15764.

32. Des Marais DJ (1997) Isotopic evolution of the biogeochemical carbon cycle dring the Proterozoic Eon. Org Geochem 27:185-193.

33. Blake TS et al. (2011) Two episodes of regional-scale Precambrian hydrothermal alteration in the eastern Pilbara, Western Australia. Precambrian Res 188:73-103.

34. Love GD, Snape CE, Carr AD, Houghton RC (1995) Release of covalently-bound alkane biomarkers in high yields from kerogen via catalytic hydropyrolysis. Org Geochem 23:981-986. 


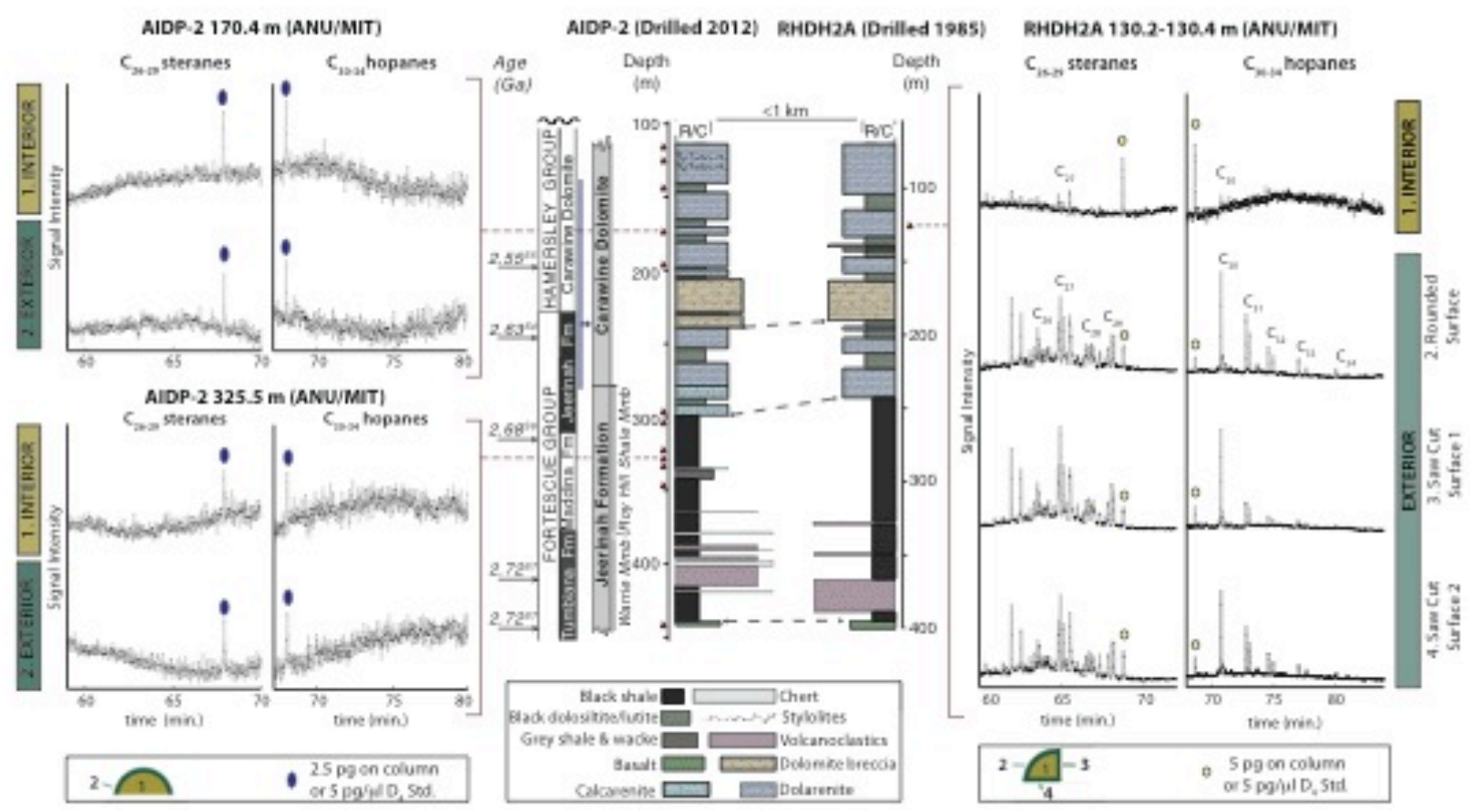

Fig. 1. Hopanes and Steranes in rock extracts from AIDP-2 compared to RHDH2A. Comparison and lithological correlation of the intersected stratigraphy of AIDP-2 (center left) and RHDH2A (center right) based on previous descriptions of RHDH2A (3, 14). The age references are listed in the SI, and red markers denote the stratigraphic depths of analyzed samples. The MRM analysis of an RHDH2A sample from the Carawine Dolomite reveals that $\mathrm{C}_{30-34}$ hopane and $\mathrm{C}_{26-29}$ sterane concentrations are higher in the core exterior compared to the interior (right). In contrast, hopanes and steranes were not detected in MRM analyses of either interiors or exteriors of a correlative sample from the Carawine Dolomite recovered at $170.4 \mathrm{~m}$ in AIDP-2 (top left) or from the underlying Roy Hill Member of the Jeerinah Formation at $325.5 \mathrm{~m}$ (bottom left). 

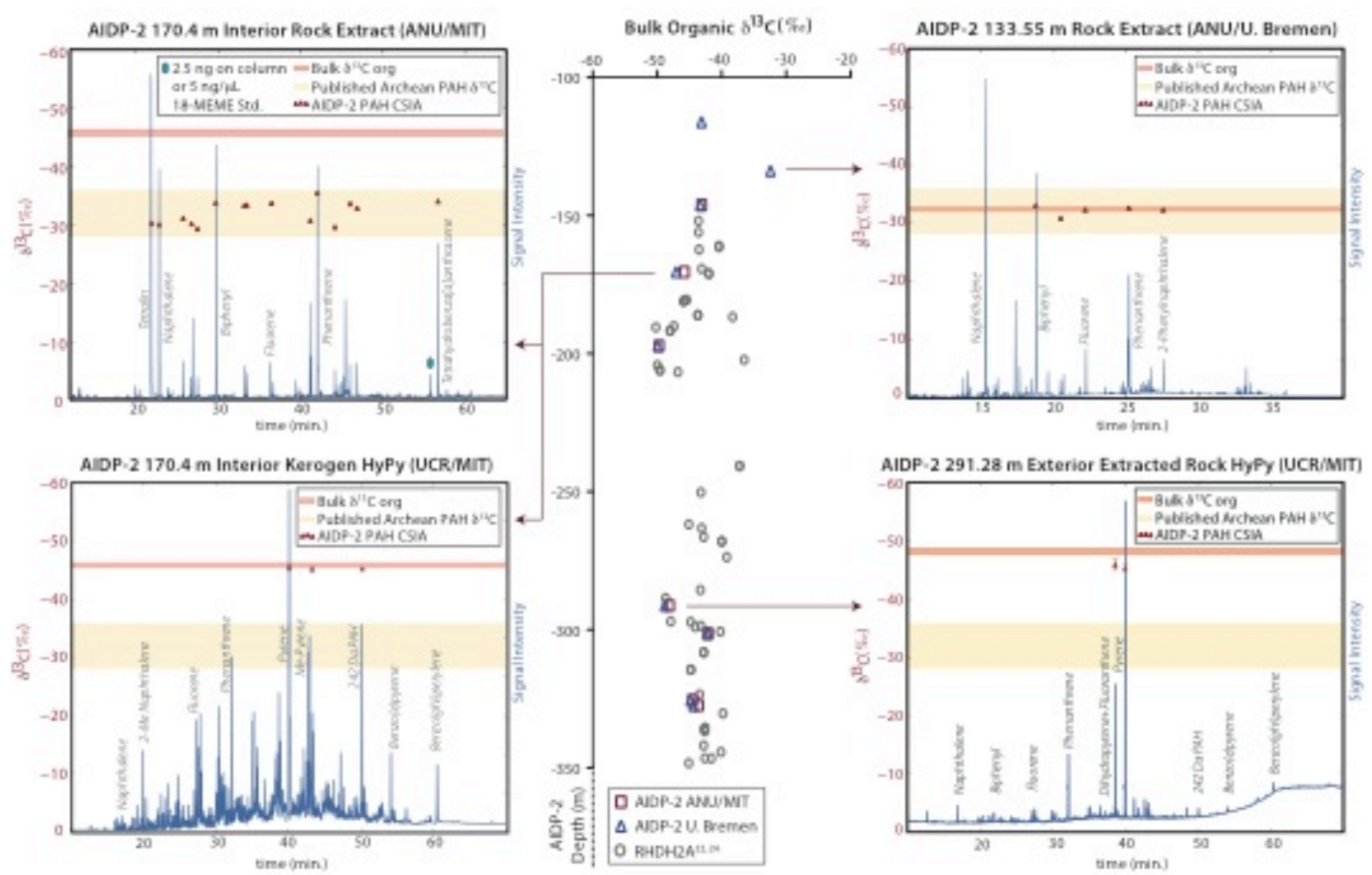

Fig. 2. Compound Specific and Bulk Organic $\delta^{13} \mathrm{C}$. Stable carbon isotopic measurements of bulk organic matter from AIDP-2 and RHDH2A $(3,31)$ are compared (center). The RHDH2A depth was converted to the AIDP-2 depth scale by a correction factor of $45 \mathrm{~m}$ due to the stratigraphic offset (see Fig. 1). Rock extract and HyPy full scan chromatograms are plotted with PAH $\delta^{13} \mathrm{C}$. AIDP-2 $133.55 \mathrm{~m}$ is the only sample that displayed an isotopic match between the extractable PAHs and the bulk organic matter (top right). The extractable $\mathrm{PAH} \delta^{13} \mathrm{C}$ from the other Carawine Dolomite samples diverged from the corresponding bulk organic matter $\delta^{13} \mathrm{C}$ (top left). The hydropyrolysate PAHs from the Carawine Dolomite and the Jeerinah Formation match the bulk organic carbon isotopic composition (bottom left and right). The range of previously reported Archean PAH $\delta^{13} \mathrm{C}$ is plotted for reference (19-21). The analytical uncertainty is plotted for the $\mathrm{PAH} \delta^{13} \mathrm{C}$ measurements, but it is smaller than the symbol in most cases. The different GC methods yield different retention times. 
Table 1. ANU/MIT hopane and sterane concentrations

\begin{tabular}{|c|c|c|c|c|}
\hline Sample & $\begin{array}{c}\text { Interior } \Sigma C_{30-34} \\
\text { Hopane } \\
\text { concentration } \\
\text { (pg/g rock) }\end{array}$ & $\begin{array}{c}\text { Interior } \Sigma C_{26-} \\
{ }_{29} \text { Sterane } \\
\text { concentration } \\
\text { (pg/g rock) }\end{array}$ & $\begin{array}{c}\text { Exterior } \Sigma \mathbf{C}_{30-34} \\
\text { Hopane } \\
\text { concentration } \\
\text { (pg/g rock) }\end{array}$ & $\begin{array}{c}\text { Exterior } \Sigma C_{26-29} \\
\text { Sterane } \\
\text { concentration } \\
\text { (pg/g rock) }\end{array}$ \\
\hline Range for blanks ${ }^{\mathrm{a}}$ & $\mathrm{nd}^{\mathrm{b}}$ to 37.9 & nd to 32.9 & nd to 37.9 & nd to 32.9 \\
\hline $\begin{array}{l}\text { AIDP-2 } 441.00 \mathrm{~m} \\
\text { (negative control) }\end{array}$ & nd & nd & 31.4 & nd \\
\hline AIDP-2 $146.2 \mathrm{~m}$ & nd & nd & 10.7 & 11.8 \\
\hline AIDP-2 $170.4 \mathrm{~m}$ & nd & nd & nd & $n d$ \\
\hline AIDP-2 $197.2 \mathrm{~m}$ & nd & nd & 50.4 & 49.4 \\
\hline AIDP-2 $291.28 \mathrm{~m}$ & nd & nd & 7.3 & 16.5 \\
\hline AIDP-2 $301.40 \mathrm{~m}$ & 5.2 & nd & 8.0 & $n d$ \\
\hline AIDP-2 $325.48 \mathrm{~m}$ & nd & $n d$ & nd & nd \\
\hline AIDP-2 $327.42 \mathrm{~m}$ & nd & nd & 38.5 & 24.3 \\
\hline AIDP-3 $98.36 \mathrm{~m}$ & nd & $n d$ & nd & nd \\
\hline AIDP-3 $130.30 \mathrm{~m}$ & nd & nd & nd & nd \\
\hline $\mathrm{RHDH}_{2 \mathrm{~A}}$ interior $^{\mathrm{c}}$ & 3.5 & 21.1 & & \\
\hline $\begin{array}{l}\text { RHDH2A sawed } \\
\text { surface } 1^{c}\end{array}$ & & & 250 & 1092 \\
\hline $\begin{array}{l}\text { RHDH2A sawed } \\
\text { surface } 2^{\mathrm{c}}\end{array}$ & & & 259 & 509 \\
\hline $\begin{array}{l}\text { RHDH2A rounded } \\
\text { surface }^{c}\end{array}$ & & & 390 & 468 \\
\hline
\end{tabular}




\subsection{Supplemental Information}

\subsubsection{Drilling}

At the beginning of the AIDP campaign, all drill rods were cleaned with soap and water, and a new drill bit was used at the beginning of the project. Furthermore, the abrasive action during the drilling of AIDP-1, which penetrated a greenschist metamorphic grade volcanic sequence that was not expected to contain hydrocarbon biomarkers, was intended to further remove residual organics that may have accumulated during previous drilling projects from the drill rods. AIDP-1 was drilled at $5^{\circ}$ with an azimuth of $55^{\circ}$ at $718123 \mathrm{E}, 7664005 \mathrm{~N}$ using water from a bore in order to recover the Coucal Formation (3.52 Ga) of the Coonterunah Subgroup (1). AIDP-2 was drilled at $77^{\circ}$ with an azimuth of $191^{\circ}$ at 51K: 275384 E, $7645330 \mathrm{~N}$ in the Ripon Hills region of the eastern Pilbara Craton, less than $\sim 1 \mathrm{~km}$ from where hole RHDH2A was collared. Water used as a drilling fluid was pumped from a permanent pool in Yilgalong Creek into a large tank, which allowed suspended sediment to settle out. Water was spiked with perdeuterated biphenyl $(1 \mu \mathrm{g} / \mathrm{L})$ and with fluorescein $(1 \mathrm{mg} / \mathrm{L})$ as synthetic tracers, which were produced on-site as stock solutions in methanol and added to the tank in regular time intervals to maintain roughly constant concentrations. The rationale was to use easy identifiable hydrophilic and hydrophobic components to assess the core penetration depth of potential contaminants that were brought into contact with the cores during the drilling process. Drilling started in reverse circulation $(\mathrm{R} / \mathrm{C})$ mode, during which no cuttings were recovered, which was performed down to a depth of $113.90 \mathrm{~m}$ to get below the weathering horizon. The hole was cleaned by flushing with copious amounts of water before diamond drilling started at HQ diameter down to a depth of $298.95 \mathrm{~m}$ and NQ diameter until $441.90 \mathrm{~m}$, at which point 
the hole was terminated. AIDP-3 was drilled at $86^{\circ}$ with an azimuth of $35^{\circ}$ at 558753 E, $7591713 \mathrm{~N}$ using water from a borehole in order to recover the Marra Mamba Iron Formation and the Jeerinah Formation. The stratigraphic columns in Figure 1 compare the AIDP-2 and RHDH2A stratigraphies where the RHDH2A stratigraphic column was constructed based on previous descriptions of $\operatorname{RHDH} 2 \mathrm{~A}(2,3)$ and previously reported age constraints (4-7).

\subsubsection{Sampling}

The drillers were instructed to avoid touching the drill core. Instead, the drilling crew shook the core material from the drilling barrels directly into the aluminum core tray. C.H. and K.L.F. washed their hands with soap prior to collecting each individual sample for organic geochemical analysis. If the sample was too long for collection, the sample was broken into a shorter piece of whole core with a cleaned geological hammer, rinsed with Type-1 ultrapure water and carefully transferred into FEP Teflon bags (Welch Fluorocarbon). The Teflon bags had been previously cleaned by boiling in Aqua Regia $\left(\mathrm{HCl} / \mathrm{HNO}_{3}:\right.$ 70/30) and repeated rinsing with organic solvents. The bags were tested for hydrocarbons prior to use in the field. All bags contained synthetic spikes $d_{34}$-hexadecane $(1 \mu \mathrm{g} / \mathrm{bag})$ and $d_{62}$-triacontane $(500 \mathrm{ng} / \mathrm{bag})$, which were added as aliquots of $1 \mathrm{~mL}$ DCM that were allowed to distribute evenly at the bottom of the bags before evaporation of the solvent. After the whole core sample was placed in the Teflon bag, a small volume of ultrapure water was added to bags to avoid sample desiccation. Samples were purged with argon before being sealed and immediately frozen in the dark at $-20^{\circ} \mathrm{C}$. This sampling procedure ensured that the samples were collected and frozen within 10-20 minutes after the drill core material was recovered from depth. Core samples remained at this temperature throughout the entire drilling campaign and during transport to Canberra, where they were stored frozen at $-20^{\circ} \mathrm{C}$. Samples were 
labeled according to the following scheme: AIDP hole number/1/order of sample collected-sample depth $(\mathrm{m})$, where the ' 1 ' indicates an ultra-clean biomarker sample (Tier-1), so the first sample taken at AIDP-2, for example, was labeled 2/1/1-116.09 m.

\subsubsection{Sawing and Sample Distribution at ANU}

The ANU/MIT team worked together at ANU in order to ensure that the AIDP samples were sawed into duplicate core halves and distributed to the individual laboratories with minimal addition of hydrocarbon contamination. A new saw (NWC 14 model SS Cut-off machine by Nortel machinery, Buffalo, NY) was purchased for the purpose of sawing the AIDP whole core samples at ANU. This saw was selected because it is constructed entirely of stainless steel and aluminum and does not use a pump to recycle water for cooling and lubrication. Instead, the saw blade was cooled and lubricated by Milli-Q water (Millipore Elix 3UV) that was gravity fed to the saw blade and subsequently collected into a tray for disposal after a single use.

Prior to sawing the AIDP samples, each step of the laboratory preparation pathway was checked for contribution of hydrocarbon contamination. At least two blanks were prepared for each laboratory step. Solvent rinses of the saw blade were collected for saw blade blanks. Combusted sand was powdered in a shatterbox (Standard Ring Mill by Rocklabs, New Zealand) using a cleaned stainless steel puck mill for puck mill blanks. Combusted ceramic tiles were loaded into clean extraction cells for Accelerated Solvent Extraction (ASE) system blanks using a Dionex ASE 200. Aliquots ( 60 mL) of dichloromethane (DCM) and hexane were evaporated for Turbovap blanks using a TurboVap LV Evaporator (Caliper Life Sciences, Inc.). Small aliquots ( 4 mL) of DCM and hexane were evaporated on a warm metal block with a gentle stream of nitrogen for evaporation block blanks. Lastly, a solvent blank was prepared. Each blank was then 
subjected to all of the downstream steps in the laboratory pathway. For a more comprehensive procedural blank, a combusted brick, which was heated at $550^{\circ} \mathrm{C}$ for 12 hours, was fed into the saw blade on a metal tray that was lined with combusted aluminum foil and sliced into small pieces. All material that was being sawed was held using combusted aluminum foil in order to minimize the introduction of surficial contamination. The combusted brick pieces were then dried in an oven at $55^{\circ} \mathrm{C}$, powdered in a puck mill, extracted by ASE, concentrated, and transferred to an insert. The set of procedural and process blanks were analyzed by gas chromatography-mass spectrometry (GC-MS) in full scan and metastable reaction monitoring (MRM) modes. The ANU/MIT laboratory and analytical methods are described in greater detail in section 2.4.4.

The blank analyses revealed that the laboratory processes downstream of sawing did not contribute detectable hydrocarbon biomarkers, specifically hopanes and steranes. The ASE extraction added diphenyl sulfone to the solvent extract. However, this compound is not a biomarker of interest, so it was disregarded as a significant hydrocarbon contaminant. In contrast, solvent rinses of multiple saw blades (Nortel Diamond wheel 12", 0.062 rim and 14", 0.07 rim and UKAM Industrial Superhard tools) yielded a mixture of hydrocarbons, including alkanes, hopanes, and steranes. These hydrocarbons continued to be detected in saw blade solvent rinses even after a number of physical and organic geochemical cleaning treatments, including scrubbing with steel wool, removing a surface layer with silicon carbide grit, solvent washing, dishwashing, and sand blasting. Moreover, the hydrocarbons associated with the saw blade were transferred to the combusted brick blank during sawing, and the amount of hydrocarbon contamination transferred from the saw blade to the sample was proportional to the newly sawed surface area of the brick. Clearly, sawing is a serious potential source of contaminant hydrocarbon detected in previously analyzed Archean 
host rocks. As most cores analyzed in previous studies were saw-cut by the drilling operators on-site, reported cumulative blanks failed to capture this important process.

After exhausting other methods of hydrocarbon contaminant removal, one of the tested saw blades was heated in a furnace to $\sim 300^{\circ} \mathrm{C}$ for 1 hour, despite potential safety concerns (i.e. delamination of the blade during sawing). The saw blade was subsequently ultrasonicated in a combusted aluminum foil envelope containing solvent. After this cleaning procedure, the saw blade solvent rinses and combusted brick extracts demonstrated that the sawing procedure was no longer a significant source of detectable hydrocarbon contaminants. In particular, the hopanes and steranes were at or below detection limit, but if a hopane or sterane was detectable the peak size had been reduced to trace levels approaching the detection limit (Table 1).

Whole core samples were treated the same way as the combusted brick blanks. Samples were sawed lengthwise into mirrored core halves, which were subsequently distributed to the participating organic geochemistry laboratories, ensuring that samples would be worked up independently in two separate laboratories. One set of core halves was worked up and analyzed at ANU in Canberra immediately after halving and thawing, implying that the cores were not subjected to potential alteration processes during subsequent storage, which could involve the formation of micro-scale desiccation cracks, pyrite oxidation, evaporation of lighter hydrocarbons from core exteriors, and additional contamination during repackaging and transport. Core halves to be distributed from the ANU laboratory to other laboratories were wrapped in combusted aluminum foil and packed into combusted glass jars, where they were padded and sealed with additional combusted aluminum foil before being screwed close. The samples were sent to Macquarie University frozen, while University of Bremen and University of California Riverside received their samples thawed. Samples were 
analyzed between three to nineteen months after drilling in the different laboratories. Unlike the samples that were analyzed directly at ANU by the ANU/MIT team, minor alteration of the distributed core halves was evident, such as oxidation of the aluminum foil due to aerial oxidation of reduced phases in the samples, such as pyrite. Sample lithologies and laboratory distributions are described in Table S1.

\subsubsection{Laboratory Methodology}

ANU \& MIT joint analysis

\section{Laboratory preparation}

The ANU and MIT teams worked together at ANU to prepare and analyze the largest set of AIDP samples, so these are the methods described in the main article. However, they are described in greater detail here. Because the frozen whole core samples were sawed in half at ANU, these samples did not undergo the additional steps of sample distribution. Instead, these samples remained frozen and purged with argon for the entire duration of storage, and they were worked up immediately or soon after the whole core was split into half. The methods for testing laboratory procedural and process blanks prior to sample analysis are described in 2.4.3. The samples that were prepared and analyzed at ANU were processed in three different rounds. At least one combusted brick blank was prepared and analyzed at the beginning of each round of sample preparation to ensure that the entire sample preparation pathway was still robust after the lapse of time (weeks or months) between sample preparation rounds. The 11 samples and corresponding 13 blanks (not including process and procedural blanks for method optimization) are listed in Table S2 in the order in which they were prepared. 
The core halves for ANU analysis were sawed into interior and exterior pieces using the same saw described in section 2.4.3. Approximately 5-10 mm slabs were removed from the core exterior, including the rounded drilling surface and the exposed core end pieces. As a result, any rock surface that could have come into contact with the Teflon bag or drilling equipment was removed and collected as the exterior sample. RHDH2A was sawed using the same saw, but because it was a quarter core that had been cut previously it was cut according to a different scheme (see the diagrams in Fig. 1). The rounded drilling surface was collected as a separate sample denoted "rounded surface". The two sawed surfaces were sawed off and collected as separate samples: "saw cut surface 1" and "saw cut surface 2." Compared to the AIDP samples, the RHDH2A sample was markedly drier and more fractured. The interior and exterior pieces were sawed into pieces that were small enough to be placed into the stainless steel puck mill at the same time that the sample was being split into interior and exterior samples.

After sawing, the interior and exterior pieces were collected as separate samples into combusted glass jars. In order to remove fine particles generated during sawing, the pieces were rinsed with Milli-Q water until the rinse water was clear. The interior and exterior pieces were dried overnight at $55^{\circ} \mathrm{C}$ in an oven with combusted aluminum foil placed loosely over the top of the glass jar. The stainless steel puck was cleaned using soap and water before being solvent rinsed with methanol, DCM, and hexane. Subsequently, at least four aliquots of combusted sand were powdered in the puck mill. The powdered sand aliquots were discarded with the exception of the last aliquot of powdered sand, which was collected as a sand blank. Next, the interior of the sample was powdered, followed by the exterior. At least four more aliquots of combusted sand were powdered between samples, where again the last sand aliquot was collected as a sand blank for the next sample. Therefore, every sample had a sand blank associated 
with it. The blank, sample interior, and sample exterior were prepared in parallel during all subsequent downstream procedures in that order.

Sample extraction was performed with a 9:1 (v:v) DCM: methanol (MeOH) solvent mixture in a Dionex ASE 200. ASE extraction cells were cleaned using soap and water and by solvent cleaning. ASE cells were then pre-extracted using combusted ceramic tile filling. The combusted sand blank was extracted in the pre-extracted ASE cell. The powdered sand was removed from the cell after extraction. The sample interior was then extracted in the same ASE cell without additional cleaning following the sand blank extraction. Similarly, the interior sample powder was removed, and the powdered sample exterior was extracted in the same ASE cell without additional cleaning. The sequential powdering and extraction of sand blank, interior, and exterior ensured that the samples were subjected to the same or cleaner conditions than the sand blank. The total lipid extract (TLE) was concentrated under a stream of purified $\mathrm{N}_{2}$ in a water bath $\left(<40^{\circ} \mathrm{C}\right)$ using a Turbovap. However, precaution was taken to ensure that the samples were never blown down to dryness during concentration and transfer steps in order to avoid losing low molecular weight compounds. Solvent cleaned activated copper granules were used to remove elemental sulfur, which was abundant in some of the extracts from the Jeerinah Formation. The concentrated, desulfurized TLE was transferred to an insert containing $0.5 \mathrm{ng}$ of $\mathrm{D}_{4}\left(d_{4}-\mathrm{C}_{29-} \alpha \alpha \alpha\right.$-ethylcholestane) standard. Each sample was then brought up to a volume of $100 \mu \mathrm{L}$ in $n$-hexane, so the concentration of the injected $\mathrm{D}_{4}$ standard was $5 \mathrm{pg} / \mu \mathrm{L}$. After samples were screened in GC/MS full scan mode, 500 ng of 18-methyl eicosanoic acid methyl ester (18-MEME) standard was added to Carawine Dolomite samples in order to quantify PAHs and diamondoids. The samples were then brought up again in $100 \mu \mathrm{L}$, so the concentration of the 18-MEME injected was $5 \mathrm{ng} / \mu \mathrm{L}$. The measured total hopane and sterane concentrations for the blanks and samples are provided in Table 1 of the main article. 


\section{GC-MS analytical conditions}

All samples were analyzed by GC/MS in full scan and MRM modes. Carawine Dolomite samples were also analyzed for aromatic steroids by selected ion monitoring (SIM). GC-MS analyses of the saturate and aromatic fractions were carried out on a Micromass AutoSpec Premier equipped with a 6890 gas chromatograph (Agilent) and a DB-5 capillary column $(60 \mathrm{~m} \times 0.25 \mathrm{~mm}$ i.d., $0.25 \mu \mathrm{m}$ film thickness) using helium as carrier gas. The MS source was operated at $260^{\circ} \mathrm{C}$ in EI-mode at $70 \mathrm{eV}$ ionization energy and with $8000 \mathrm{~V}$ acceleration voltage. Samples were injected in splitless mode into a PTV injector was ramped from $60^{\circ} \mathrm{C}$ (hold for $0.1 \mathrm{~min}$ ) to $300^{\circ} \mathrm{C}$ at $260^{\circ} \mathrm{C} / \mathrm{min}$ and held at the maximum temperature for the remaining duration of the analysis. For full-scan analyses, the $\mathrm{GC}$ oven was programmed at $60^{\circ} \mathrm{C}(4 \mathrm{~min})$, heated to $315^{\circ} \mathrm{C}$ at $4^{\circ} \mathrm{C} / \mathrm{min}$, with a final hold time of $22.3 \mathrm{~min}$. The AutoSpec full-scan duration was $0.7 \mathrm{~s}$ plus $0.2 \mathrm{~s}$ interscan delay over a mass range of 55-600 Da. For MRM and SIM, the GC oven used the same temperature program except that it held the final temperature for 32.3 minutes. The MRM channel that included hopane and sterane transitions had a cycle time of 918.4 milliseconds and an inter scan delay of 0.1 seconds. All samples were injected in $n$-hexane ( 0.5 or $1 \mu \mathrm{L}$ out of a total volume of $100 \mu \mathrm{L})$ to avoid deterioration of chromatographic signals by $\mathrm{FeCl}_{2}$ build-up in the MS ion source caused by the use of halogenated solvents (8).

\section{Compound-specific isotope analyses at Geoscience Australia of extractable PAH}

The carbon isotopic composition of individual hydrocarbons was determined by gas chromatography-isotopic ratio mass spectroscopy (GC-IRMS). The analytical system included a Thermo Finnigan Trace GC interfaced via a Thermo Finnigan GC

Combustion III to a Thermo Scientific MAT 253 isotope-ratio mass spectrometer. Depending on solvent volume, samples were injected on-column either manually or 
automatically (GC PAL autosampler). Helium was used as the carrier gas at a constant flow of $2 \mathrm{~mL} / \mathrm{min}$. The GC capillary column was a DB-5 (60 m x $0.32 \mathrm{~mm}$ i.d., $0.25 \mu \mathrm{m}$ film thickness) and the GC oven was programmed at $40^{\circ} \mathrm{C}$ for $10 \mathrm{~min}$, heated to $310^{\circ} \mathrm{C}$ at $4^{\circ} \mathrm{C} / \mathrm{min}$ and held at the final temperature for up to $10 \mathrm{~min}$. Data was acquired and processed using the software package ISODAT 3.0. The system was calibrated and corrected for instrument drift by co-injecting internal standards of perdeuterated $n$ alkanes $\left(\mathrm{C}_{16} \mathrm{D}_{34}, \mathrm{C}_{20} \mathrm{D}_{42}, \mathrm{C}_{24} \mathrm{D}_{50}\right.$; Chevron) with known isotopic compositions. All $\delta^{13} \mathrm{C}$ values are reported in \%o relative to the Pee Dee Belemnite (VPDB) standard. Isotopic analyses were carried out in duplicates with reported data achieving an error within $\pm 0.5-1.0 \%$ for polyaromatic hydrocarbons. The results are included in Table S6.

\section{Total organic carbon and bulk organic $\delta^{13} \mathrm{C}$}

Nearly $0.5 \mathrm{~g}$ of unextracted interior sample powder was weighed into a test tube for decarbonation using $3 \mathrm{~N}$ hydrochloric acid $(\mathrm{HCl})$. The samples were allowed to react at room temperature over night before they were centrifuged and decanted. Each sample was tested to ensure that additional $\mathrm{HCl}$ did not generate further reaction. The residual material was rinsed with Milli-Q water until the supernatant was $\mathrm{pH}$ neutral. The samples were then dried over night in a $60^{\circ} \mathrm{C}$ oven. The remaining sample material was weighed in order to calculate the percent carbonate. The sample material was further homogenized using a glass rod before being transferred to combusted $4 \mathrm{~mL}$ glass vials.

The total organic carbon (TOC) and bulk organic $\delta^{13} \mathrm{C}$ was performed in the Fallon laboratory at ANU. A preliminary test run using $10 \mathrm{mg}$ of sample was performed in order to estimate how much carbon was in each sample. Based on the test results, the amount of sample weighed into the tin capsule was modified in order to introduce approximately $0.25 \mathrm{mg}$ of carbon into the mass spectrometer for every sample. For the final analysis, $\sim 4 \mathrm{mg}$ of vanadium pentoxide (Carlo Erba) was weighed into each 
sample and standard capsule to enhance combustion efficiency. Each sample was prepared in duplicate. An empty tin capsule was analyzed in between samples to assess and prevent sample carry over. Eighteen polyethylene standards (IAEA-CH-7; 85.7\% C; $\delta^{13} \mathrm{CVPDB}=-32.15+/-0.05 \%$ ) were prepared and used to bracket groups of five samples and five tin capsules. USGS-40 (L-glutamic acid; $\delta^{13} \mathrm{CvPDB}=-26.389+/-0.04 \% ; 40.8 \% \mathrm{C}$ ), USGS-41 (L-glutamic acid; $\delta^{13} \mathrm{C}=+37.63+/-0.05 \%$ ), and IAEA-600 (caffeine; $\delta^{13} \mathrm{CvpDB}=-$ $27.771+/-0.043 \% ; 49.48 \%$ C) were prepared in duplicate for secondary standards that were analyzed at the beginning and end of the sample sequence. A set of six glycine standards ( $32 \% \mathrm{C} ; \delta^{13} \mathrm{CVPDB}=-48.26 \%$ ) with masses ranging from $0.15 \mathrm{mg}$ to $0.9 \mathrm{mg}$ of $\mathrm{C}$ were used to test the linearity of the measurement. A SerCon 20-22 continuous flow isotope ratio mass spectrometer (CS-IRMS) coupled to an Automated Nitrogen Carbon Analyser- Gas Solids and Liquids Elemental Analyzer (ANCA-GSL EA) was used to analyze the sample set in continuous flow mode.

The average carbon isotopic value of the IAEA-CH-7 polyethylene standard, which was measured throughout the sample sequence, was within $0.15 \%$ of the accepted $\delta^{13} \mathrm{CVPDB}_{\mathrm{V}}$ value and the average carbon percentage was within $0.2 \%$ of the accepted carbon percentage. The TOC and bulk $\delta^{13} \mathrm{C}$ results are given in Table S5. The bulk $\delta^{13} \mathrm{C}$ is the average of the $\delta^{13} \mathrm{C}$ sample duplicates with the difference between the average $\delta^{13} \mathrm{C}$ and individual measurements reported as the corresponding standard deviation. The uncertainty of the TOC measurements cannot be determined with certainty since they rely on the uncertainty of the carbonate percentage calculations, but the duplicate measurements of the decarbonated material were never greater than $0.5 \%$ from the average value.

Analyses at U. Bremen \& MPI Jena 


\section{Laboratory preparation}

Before proceeding to analyze samples, the cleanliness of the laboratory procedure was tested with a series of process blanks, which tested individual steps of the workup path, and cumulative procedural blanks, which are subjected to the full equivalent sample workup. For procedural blanks we used pieces of brick that were cut to sticks with dimensions of ca. $3 \times 3 \times 10 \mathrm{~cm}$, wrapped in aluminum foil and baked at $500^{\circ} \mathrm{C}$ for 10 hours to remove potential organic contaminants by combustion. Given the focus on diagnostic biomarker hydrocarbons, the cleanliness was determined by target compound analysis for hopanes and steranes. While process blanks with biomarker levels below detection limit could be achieved, this could not be accomplished for procedural blanks. Workup of core samples was initiated once we consistently achieved a level of steroid contamination of $<100 \mathrm{fg}$ per chromatographic peak and levels of hopanoid contamination < ca. $300 \mathrm{fg}$ per peak over three consecutive procedural blanks (see section 2.4.5. for discussion of blank concentrations). The persistent but trace contaminants had a non-fossil signature (e.g. only $\mathrm{C}_{27} \alpha \alpha \mathrm{R}$ amongst the regular steranes).

Six core samples and two brick blanks were sawed into interior and exterior portions, removing ca. $1 \mathrm{~cm}$ of material from all core edges, by using an all-stainless steel trim saw that was fitted with a diamond-rimmed blade. The blade had undergone multiple cleaning steps that involved repeated ultrasonication in organic solvents and baking at $400^{\circ} \mathrm{C}$ before an acceptable level of blade cleanliness was achieved. Core samples were held with pieces of baked aluminum foil and the blade was lubricated with a continuous stream of doubly purified, deionized and organic-clean water $(0.055 \mu \mathrm{S} / \mathrm{cm}$; 2-7 ppb TOC) that was not recirculated. Core pieces were subsequently transferred to beakers and dried on a hot plate at $60^{\circ} \mathrm{C}$ until dry and tightly covered with aluminum foil for temporary storage. Subsequently, core interiors and one brick blank were fully 
processed before workup of core exteriors was initiated. Fragments were wrapped in a piece of thick $(50 \mu \mathrm{m})$ aluminum foil and crushed by the impact of a DCM-cleaned hammer on a DCM-cleaned steel plate. Fragments were transferred to clean aluminumfoil covered beakers, where they were stored until powdering. The latter was performed in a Siebtechnik Shatterbox equipped with a stainless steel puck mill that was cleaned by baking at $500^{\circ} \mathrm{C}$. All core interiors were powdered first, followed by all core exteriors, where the puck and container was baked in between. For both interiors and exteriors, the sequence of powdering was brick blank, negative control chert sample, sample 2/1/014, sample 2/1/10, sample 2/1/008, sample 2/1/007, sample 2/1/004, and sample 2/1/001. The powdered samples were transferred to glass jars for long-term storage (Table S3). In between samples, the puck and milling container were cleaned by grinding combusted quartz sand four times and rinsing with dichloromethane. Sample powder aliquots of ca. $8 \mathrm{~g}$ were transferred into pre-cleaned Teflon tubes, in which they were microwave-extracted (CEM Mars-6; 30 minutes at $110^{\circ} \mathrm{C}$ ) under stirring with 50 $\mathrm{mL}$ of DCM (Merck, UniSolv grade). All of the Teflon tubes had previously undergone the extraction of process blanks, which confirmed their cleanliness. After centrifugation the extracts were decanted and the extraction step was repeated. Pooled extracts were evaporated at $40^{\circ} \mathrm{C}$ and 800 mbar to a residual volume of $1 \mathrm{~mL}$. The extracts were replenished with $10 \mathrm{~mL}$ cyclopentane (Merck, OmniSolv grade; double distilled before use) before being reduced again to a volume of $1 \mathrm{~mL}$ under the same conditions. The concentrated extracts were transferred in cyclopentane to total-recovery GC-vials spiked with $20 \mathrm{ng}$ of $d_{14-p}$-terphenyl and $500 \mathrm{pg}$ of $d_{4-}-\alpha \alpha \mathrm{R}$-cholestane. Subsequently core exteriors were processed following the same protocol, using the same Teflon tubes that were employed for corresponding core interior samples. No preparative chromatographic separation of compound classes was undertaken to minimize the potential addition of contaminants during the laboratory workup. 


\section{GC-MS analytical conditions}

Extracts were analyzed in full scan mode using an Almsco BenchTOF- $d x$ mass spectrometer coupled to a Thermo Trace GC Ultra. Volumes of 2 out of ca. $1000 \mu \mathrm{L}$ (samples dissolved in c-pentane) were injected in splitless mode (PTV injector ramped from $60^{\circ}$ to $315^{\circ} \mathrm{C}$ with $14.5^{\circ} / \mathrm{sec}$ at $72.4 \mathrm{psi}$ ) and separated on a VF-1MS capillary column (40 m x $0.15 \mathrm{~mm}$ I.D. $\times 0.15 \mu \mathrm{m}$ film thickness) using Helium 5.0 carrier gas at a constant flow rate of $1.4 \mathrm{~mL} /$ minute and a temperature program starting at $60^{\circ} \mathrm{C}(2$ minutes), ramped to $325^{\circ} \mathrm{C}$ at $4.5^{\circ} / \mathrm{min}$ and held at final temperature for 15 minutes. A constant split flow (apart from 1 minute of splitless flow during sample injection) of 100:1 ensured a minimum of atmospheric components in the carrier gas. Ionization was achieved by electron impact at $70 \mathrm{eV}$ and $250^{\circ} \mathrm{C}$ with a filament current of ca. 4 A. Data was measured from m/z 30-800 but was only recorded from m/z 50-550 at 1000 mass resolution using 2469 scans per scanset and a scanset period of $250 \mathrm{~ms}$. Compounds

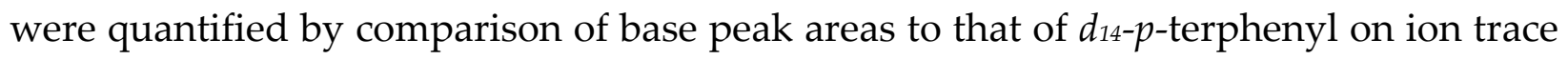
$\mathrm{m} / \mathrm{z} 244$ without correcting for differential response factors. PAH and diamondoid ratios and calculated vitrinite reflectances are given in Tables S8 and S9, respectively.

Target compound analysis for hopanoids and steroids was performed on a Thermo Quantum XLS Ultra triple quadrupole MS coupled to a Thermo Trace GC Ultra. Volumes of 6 out of ca. $1000 \mu \mathrm{L}$ (samples dissolved in c-pentane) were injected on column at $70^{\circ} \mathrm{C}$. We used a $60 \mathrm{~m}$ DB-5MS capillary column $(0.25 \mathrm{~mm}$ I.D., $0.25 \mu \mathrm{m}$ film thickness) linked to a $10 \mathrm{~m}$ deactivated pre-column (0.53 $\mu \mathrm{m}$ I.D.). Helium 5.0 was used as a carrier gas with a constant flow of $1.3 \mathrm{~mL} / \mathrm{min}$. The oven was held isothermal at $70^{\circ} \mathrm{C}$ for 5 minutes before ramping with $4^{\circ} /$ minute to $335^{\circ} \mathrm{C}$, where it was held isothermal for 9 minutes. Ionization was achieved by electron impact at $70 \mathrm{eV}$ and $250^{\circ} \mathrm{C}$, with an emission current of $50 \mu \mathrm{A}$. Q1 and Q3 were each operated in $0.7 \mathrm{Da}$ resolution with a cycle time of 0.5 seconds. Q2 was operated with Argon 5.0 collision 
gas at a pressure of $1.1 \mathrm{mTorr}$ and varying collision voltages depending on the target analyte. Compounds were quantified relative to $d_{4}$-cholestane without correcting for differential response factors.

\section{Compound-specific stable carbon isotope analyses of extractable PAH at $U$. Bremen}

Compound-specific isotope analyses (CSIA) were performed at the University of Bremen on a Thermo Finnigan MAT-252 coupled to a Thermo Trace GC via a modified combustion interface using $\mathrm{Ni}$ wire and oxygen trickle flow at $1000^{\circ} \mathrm{C}$. Volumes of 5 out of $25-50 \mu \mathrm{L}$ (samples dissolved in $n$-hexane) were injected on-column at $70^{\circ} \mathrm{C}$. A $60 \mathrm{~m}$ DB-5MS capillary column $0.32 \mathrm{~mm}$ I.D., $0.25 \mu \mathrm{m}$ film thickness) linked to a $2 \mathrm{~m}$

deactivated pre-column (0.53 $\mu \mathrm{m}$ I.D.) was used with a constant flow $(1.2 \mathrm{~mL} / \mathrm{min})$ of Helium 5.0 as a carrier gas. The oven was held isothermally for 5 minutes, then ramped to $230^{\circ} \mathrm{C}$ at $2.5^{\circ} \mathrm{C} /$ minute, to $300^{\circ} \mathrm{C}$ at $5^{\circ} \mathrm{C} /$ minute and to $330^{\circ} \mathrm{C}$ at $15^{\circ} \mathrm{C} /$ minute, where it was held isothermally for 15 minutes. Stable carbon isotope ratios were determined relative to a co-injected $\mathrm{CO}_{2}$ standard that was cross-calibrated relative to a reference mixture of $n$-alkanes. Data are presented in the conventional $\delta^{13} \mathrm{C}$ notation as permil deviations from the VPDB standard. The reliability of small chromatographic peaks was verified down to an intensity of ca. $30 \mathrm{mV}$ by analyzing increasingly diluted samples of the saturated hydrocarbon fraction of the NSO-1 geochemical standard (Oseberg oil; Norwegian Petroleum Directorate). Results are given in Table S6.

Preparation and analyses of 2/1/002

Sample 2/1/002, representing pyrobituminous material occurring in stylolitic structures, was worked up during a visit to ANU immediately after the drilling campaign. The sample was fragmented into smaller portions by impact of a hammer after being wrapped in combusted aluminum foil. Individual pieces were transferred to a precombusted mortar and powdered with a pestle. The sample was extracted twice by 
ultrasonic agitation in DCM and concentrated to near dryness on a heating block, after which it was resuspended in hexane and further concentrated to fully remove remnant DCM. No removal of elemental sulfur or column chromatography was performed to minimize potential for contamination. The concentrated sample exhibited a faint yellow hue and was analyzed in full scan and MRM mode on a Micromass AutoSpec Premier coupled to an Agilent 6890 GC at ANU using the same parameters described in section 4.1.2. The sample was further concentrated and subjected to GC-irm-MS analysis at Geoscience Australia using the same parameters described in section 4.1.3.

\section{$H$ and $C$ elemental analysis}

Elemental proportions of hydrogen and carbon in kerogen were determined on dried kerogen powders after digestion of the mineral matrix. Aliquots of ca. $5 \mathrm{~g}$ of sample powder, taken from core exterior portions, were wetted with methanol and treated twice with aqueous $\mathrm{HCl}$ (first $6 \mathrm{~N}$, then $12 \mathrm{~N}$ ) under constant stirring. The acid was decanted and samples were rinsed 8-10x with Type-1 ultrapure water, at which point the $\mathrm{pH}$-value of the water (ca. 4.5) remained stable. After drying and gravimetric determination of carbonate loss, samples were powdered with a Teflon rod, again wetted with methanol and treated twice with aqueous HF (48\%) under constant stirring for $>24$ hours each. Supernatant acid was decanted and the digestion residues were rinsed with Type-1 water (10x). After drying and powdering, kerogens were analyzed at the MPI-BGC in a Vario EL II cube CNHS elemental analyzer (Elementar Analysensysteme) by TCD detection after oxidation of the kerogen to $\mathrm{CO}_{2}$ and $\mathrm{H}_{2} \mathrm{O}$ at $1150^{\circ} \mathrm{C}$. Sample powders were additionally dried at $110^{\circ} \mathrm{C}$ over night and subsequently weighed with a $\mathrm{WO}_{3}$ catalyst, first into silver cups (to react with any remnant fluoride) and then into secondary tin cups (given the exothermal reaction of Sn). Helium was used as a carrier gas, spiked with an $\mathrm{O}_{2}$ pulse for flash combustion. Oxygen was eliminated from the gas stream by passing through active copper at $850^{\circ} \mathrm{C}$. Remnant 
halogens were trapped on silver wool and any persistently remnant fluoride was trapped on magnesium oxide. Results are reported in Table S5.

\section{Bulk Organic $\delta^{13} \mathrm{C}$ analyses}

The stable carbon isotope ratios of macromolecular organic matter were determined on unextracted rock powder after removal of carbonates using $6 \mathrm{~N}$ and $12 \mathrm{~N}$ hydrochloric acid. The neutralized, dried and homogenized residues were weighed into tin cups and analyzed after online combustion to $\mathrm{CO}_{2}$ in an in-house refurbished and modified Finnigan MAT Delta C prototype isotope ratio monitoring (irm) MS, coupled to a Carlo Erba EA-1100 via a ConFlo III interface. The stable carbon isotopic values are reported in the permil notation relative to VPDB, after calibration by the NBS-22 reference standard. Results are reported in Table S5.

\section{Macquarie University}

Prior to AIDP sample preparation, laboratory hydrocarbon contribution was assessed using an outcrop sample that was collected from a basalt lava flow of the Maddina Formation. The outcrop samples were combusted in the laboratory at $400^{\circ} \mathrm{C}$ for three hours before being used for laboratory system blanks. The blank was processed by the same procedures described below for the AIDP samples. After the blanks were assessed, five biomarker target samples from AIDP-2 and AIDP-3 were examined for preserved hydrocarbons, and one dolerite sample from AIDP-1 was processed as a negative control sample (Table S4).

Drill cores were received frozen from the ANU/MIT team and remained frozen until they were sawed. The samples were manipulated through aluminum foil and never contacted other materials except for a clean saw blade (BUEHLER, BUE11-4267; $178 \mathrm{~mm}$ 
diameter; $0.6 \mathrm{~mm}$ thickness) and Milli-Q water that was used for cooling and lubrication. Aluminum foil and glassware were combusted at $400^{\circ} \mathrm{C}$ for at least three hours before use. The saw blade was combusted twice at $300^{\circ} \mathrm{C}$ for three hours and sonicated with $\mathrm{MeOH}$ and DCM several times to eliminate surface contamination before any processing. Between each sample, the saw blade was sonicated with Milli-Q water (1x), $\mathrm{MeOH}(600 \mathrm{~mL} ; 1 \mathrm{x})$ and $\mathrm{DCM}(600 \mathrm{~mL} ; 2 \mathrm{x})$, and $200 \mathrm{~mL}$ of the last $\mathrm{DCM}$ solvent rinse was analyzed by GC-MS to measure the organic residues. During sawing, the Milli-Q water was not recycled but went directly to the waste after a single use. Although clear signs of weathering were not observed, vibration during cutting cracked some samples, indicating the presence of micro-fractures. A few millimeters of drill core exterior was sawed off to distinguish indigenous hydrocarbons from surficial drilling and experimental contaminants. The interior and exterior pieces were rinsed separately with Milli-Q water (1x), MeOH ( 100 mL; 1x), and DCM ( 100 mL; 2x). The interior and exteriors were powdered to $<200$ mesh grain size in a ring-mill (Rocklab). After each sample, the ring mill was scrubbed with a metal brush and rinsed with tap water (1x), $\mathrm{MeOH}(30-40 \mathrm{~mL} ; 2 \mathrm{x})$, and DCM $(30-40 \mathrm{~mL} ; 3 \mathrm{x})$. The ring mill was further rinsed with DCM (30-40 mL; at least 3x) mechanically using a milling head. The last solvent rinse was analyzed by GC-MS to measure the organic residues. The solvent rinses that were analyzed by GC-MS to measure the organic residues provided a qualitative sense of contamination, rather than a quantitative measure of contamination, because solvent volumes were not measured and constant. Hydrocarbons were extracted by sonication with 9:1 (v:v) DCM:MeOH. Column fractionation was not performed because of low abundances and potential introduction of contamination. The extracted organic matter was spiked with three compounds as internal standards by adding $1 \mathrm{~mL}$ of a DCM solution containing about $50 \mathrm{ng}$ of each: anthracene- $d_{10}$ (98 atom \%D, Isotec), $p$ terphenyl- $d_{14}$ (98 atom \%D, Isotec), and tetraeicosane- $d_{50}$ (98 atom $\% \mathrm{D}$, Isotec). The volume of solvent containing the extracted organic matter was further reduced on a hot 
plate at $60-70^{\circ} \mathrm{C}$ under a gentle nitrogen flow, until approximately $50 \mu \mathrm{L}$ remained. Desulfurisation was not carried out except for sample 2/1/007 (291.28 m), which had a large amount of sulfur. In that case, $5 \mathrm{~g}$ of activated copper particles (metal turnings, TG, Chem-Supply) were added to the extracted organic matter from sample 2/1/007, and stirred for a few minutes. This simple procedure was enough to remove most sulfur so that reflux of the solution was not performed. The extracted organic matter was analyzed by GC-MS.

\section{Macquarie GC/MS analytical conditions}

GC-MS analysis was carried out on an Agilent GC (6890N) coupled to an Agilent Mass Selective Detector (5975B). An aliquot of $1 \mu \mathrm{L}$ of the $50 \mu \mathrm{L}$ solution was injected into a PTV inlet operating in splitless mode with a J\&W DB5 MS column (length $60 \mathrm{~m}$, inner diameter $0.25 \mathrm{~mm}$, film thickness $0.25 \mu \mathrm{m})$. The inlet was ramped from $35^{\circ} \mathrm{C}(3 \mathrm{~min}$. isothermal) to $310^{\circ} \mathrm{C}(0.5 \mathrm{~min}$. isothermal $)$ at a rate of $700^{\circ} \mathrm{C} / \mathrm{min}$. Helium was used as the carrier gas $(1.5 \mathrm{~mL} / \mathrm{min}$.$) , and the temperature of the \mathrm{GC}$ oven was ramped from $30^{\circ} \mathrm{C}$ (2 min. isothermal) to $310^{\circ} \mathrm{C}\left(30 \mathrm{~min}\right.$. isothermal) at a rate of $4^{\circ} \mathrm{C} / \mathrm{min}$. The MS data were acquired in SIM mode. Semi-quantitative analyses were performed using the tetraeicosane- $d_{50}$ internal standard without taking into account response factors. The other two standards were used to check sensitivity and reproducibility of the measurement, but were not used for the quantification.

In order to analyze for small concentrations of hopanes and steranes, samples were further analyzed by either a Thermo Trace Ultra GC interfaced with a high resolution Thermo DFS GC-MS system or a GC-linked Micromass Autospec Premier. The samples AIDP-1/1/004-186 $\mathrm{m}$ and AIDP-3/1/005-130.3 $\mathrm{m}$ were analyzed by the Thermo DFS instrument. However, the DFS instrument experienced a technical problem, so the samples AIDP-2/1/3-146.2 m, AIDP-2/1/007-291.28 m, and AIDP-2/1/011-327.42 m were 
analyzed by the Autospec in the Brocks lab at ANU.

In the DFS measurements, GC separation was carried out on a J\&W DB-5 MS capillary column (length $60 \mathrm{~m}$, inner diameter $0.25 \mathrm{~mm}$, film thickness $0.25 \mathrm{um}$ ). Helium was used as the carrier gas $(1.5 \mathrm{~mL} / \mathrm{min}) .1 \mu \mathrm{L}$ of the extracted organic matter solution was injected into an inlet operating at $260^{\circ} \mathrm{C}$ in splitless mode. The MS was tuned to 1,000 resolution (electron energy $70 \mathrm{eV}$; source temperature $280^{\circ} \mathrm{C}$ ). The oven was ramped from $40^{\circ} \mathrm{C}\left(2 \mathrm{~min}\right.$ isothermal) to $200^{\circ} \mathrm{C}$ at a rate of $4^{\circ} \mathrm{C} / \mathrm{min}$, then to $310^{\circ} \mathrm{C}(30 \mathrm{~min}$ isothermal) at a rate of $2^{\circ} \mathrm{C} / \mathrm{min}$. The MS data were acquired in SIM mode.

The Autospec measurements were carried out on a Micromass AutoSpec Premier GCMS equipped with an Agilent 6890 GC and a J\&W DB5MS capillary column (length 60 $\mathrm{m}$, inner diameter $0.25 \mathrm{~mm}$, film thickness $0.25 \mu \mathrm{m})$. Helium was the carrier gas (1.5 $\mathrm{mL} / \mathrm{min}) .1 \mu \mathrm{L}$ of the extracted organic matter solution was injected into a PTV injector in splitless mode. The injector was ramped from $60^{\circ} \mathrm{C}(2 \mathrm{~min}$, isothermal $)$ to $300^{\circ} \mathrm{C}$. The MS source was operated at $260^{\circ} \mathrm{C}$ in EI-mode at $70 \mathrm{eV}$ ionization energy, and with an acceleration voltage of $8000 \mathrm{~V}$. The GC oven was ramped from $60^{\circ} \mathrm{C}$ (4 min, isothermal) to $315^{\circ} \mathrm{C}\left(32.3 \mathrm{~min}\right.$ isothermal) at a rate of $4^{\circ} \mathrm{C} / \mathrm{min}$. The MS data were acquired in SIM mode.

The concentration of biomarkers obtained by the DFS measurements were scaled to fit the Autospec data, by comparing the peak areas of the internal standard in the procedural blank obtained by the DFS and the Autospec. The procedural blank was measured by both instruments to measure the scaling factor from the DFS system to the Autospec system. Semi-quantitative analyses were performed using the $p$-terphenyl- $d_{14}$ internal standard, not taking into account any response factors. The tetraeicosane- $d_{50}$ was not used due to its low $\mathrm{m} / \mathrm{z}$ value relative to that of biomarkers. 


\section{University of California-Riverside}

\section{Laboratory procedures}

Immediately after receiving the AIDP core samples, the glass jars holding the core halves were inspected before placing them in the freezer for storage. Drill cores were kept frozen until just before saw cutting. AIDP sample preparation was carried out with extreme care where direct handling of core pieces was done only with pre-combusted aluminum foil. The aluminum foil and all glassware were combusted at $550^{\circ} \mathrm{C}$ for at least six hours. Before use, the glassware was further cleaned by rinsing twice with methanol, dichloromethane and hexane solvents. Sawing of core portions was performed using a new metal bond diamond saw blade, which was rinsed thoroughly with Milli-Q water (3x), methanol (3x), dichloromethane $(3 x)$ and hexane $(3 x)$ before each sample cutting to eliminate surface contamination. The Milli-Q water that was used for rinsing, cooling, and lubrication during cutting was pre-extracted with DCM (3x) to minimize potential organic contamination. The last DCM-wash was collected as a laboratory blank. During cutting, the Milli-Q water was changed regularly. Approximately 3-5 mm layer of the exterior of each drill core sample was sawed off, leaving an interior core portion. The exterior and interior portions were cut into smaller pieces. The surfaces of these smaller core pieces were cleaned by ultrasonication (three times) for two minutes using sequential solvents rinses with methanol, dichloromethane then hexane, before being placed in separate pre-combusted glass jars for powdering.

The inner and outer core portions were powdered separately using a SPEX 8515 
shatterbox equipped with a zirconia puck and dish. The zirconia puck and dish were first thoroughly cleaned by sequentially rinsing with methanol, dichloromethane then hexane ( $3 x$ for each solvent). The mill was further cleaned by powdering at least four aliquots of pre-combusted $\left(450^{\circ} \mathrm{C}\right)$ quartz sand. The last aliquot of powdered sand was collected as a sand blank, which was processed through all of the same subsequent laboratory steps as for the AIDP rock powders. The inner sample portion was powdered first followed by the exterior part of that same sample.

Between $30-70 \mathrm{~g}$ of AIDP rock powders, worked up alongside combusted sand powders as procedural blank, were extracted for bitumens by Soxhlet extraction using pre-extracted cellulose extraction thimbles with an azeotropic solvent mixture of 93:7 (v/v) dichloromethane/methanol for 48 hours. The extracts were carefully dried down to near dryness at ambient temperature in a fumehood to minimize evaporation of volatile organics. Pre-cleaned $\mathrm{Cu}$ pellets were added to the solvent extracts to remove any elemental sulfur. Pre-extracted rock powders were retained and stored in precombusted glass jars for subsequent hydropyrolysis treatment to fragment kerogenbound organic constituents.

Whole rock powders from UCR were analyzed for TOC using a LECO C230 instrument by GeoMark Research. Leco TOC analysis requires decarbonation of the rock sample by treatment with hydrochloric acid $(\mathrm{HCl})$. This is achieved by treating the samples with concentrated $\mathrm{HCl}_{(\mathrm{aq})}$ for at least two hours. The samples were then rinsed with water and flushed through a filtration apparatus to remove the acid. The filter was then removed, placed into a LECO crucible and dried in a low temperature oven $\left(110^{\circ} \mathrm{C}\right)$ for a minimum of 4 hours. Samples were also weighed after this process in order to obtain a percent carbonate value based on weight loss. The LECO C230 instrument was calibrated with standards having known carbon contents. This was completed by 
combustion of these standards by heating to $1200^{\circ} \mathrm{C}$ in the presence of oxygen. Standards were analyzed every 10 samples to check the variation and calibration of the analysis. The acceptable standard deviation for TOC is 3\% variation from established value.

\section{UC Riverside Hydropyrolysis (HyPy) analytical conditions}

Continuous-flow hydropyrolysis experiments were typically performed on 1000-2000 mg of pre-extracted sedimentary rock powders or kerogen concentrate powders, as described previously (9). The isolation of kerogen concentrates for three carbonate-rich samples (2/1/001 inner, 2/1/003 inner and 2/1/004 inner) was conducted on solventextracted rock residues by standard hydrochloric acid $(\mathrm{HCl})$ extraction procedures. Further treatment of the isolated kerogens involved extraction with dichloromethane to remove any residual bitumen, such as bitumen 2, using a Microwave Accelerated Reaction System (CEM corp.) with dichloromethane and methanol $(9: 1 \mathrm{v} / \mathrm{v})$ at $100^{\circ} \mathrm{C}$ for 15 minutes. For the other five samples (2/1/007 outer, 2/1/010 inner, 2/1/011 outer, 3/1/004 outer, 3/1/005 outer), pre-extracted rock powders were used.

The pre-extracted rock/kerogen powders were heated in a stainless steel (316 grade) reactor tube from ambient temperature to $250^{\circ} \mathrm{C}$ at $300^{\circ} \mathrm{C} / \mathrm{min}$ then to $520^{\circ} \mathrm{C}$ at $8^{\circ} \mathrm{C} / \mathrm{min}$. A hydrogen sweep gas flow of $6 \mathrm{dm}^{3} / \mathrm{min}$, measured at ambient temperature and pressure, through the reactor bed ensured that the residence times of generated volatiles was on the order of only a few seconds. Products were collected in a silica gel trap cooled with dry ice and adsorbed products were separated into polarity fractions using silica gel adsorption chromatography. The extracted rock powders or kerogens from eight different AIDP samples were subjected to HyPy treatment, with three of these performed in duplicate (Table S10). 
To ensure a clean system for HyPy, at least one cleaning run was performed before each sample run whereby the apparatus was heated to $520^{\circ} \mathrm{C}$ using a rapid heating rate $\left(300^{\circ} \mathrm{C} / \mathrm{min}\right)$ under high hydrogen pressure conditions. This included the steel wool plug used to support the sample powder in the optimum position in the heated zone of the reactor tube. Experimental blanks, using annealed silica gel in the reactor tube instead of a sample, were regularly performed using the same temperature program as for core samples and the products monitored and quantified to ensure that trace organic contamination levels were acceptably low.

\section{UC Riverside GC/MS analytical conditions}

For rock bitumens and hydropyrolysates, full scan GC-MS was performed over a mass range of 50 to $600 \mathrm{Da}$ on two different instruments. Initial screening was performed on an Agilent 5975C inert MSD mass spectrometer interfaced to an Agilent 7890A GC, equipped with a J\&W DB-1MS capillary column $(60 \mathrm{~m} \times 0.32 \mathrm{~mm}, 0.25 \mu \mathrm{m}$ film). The temperature program for GC-MS full scan was $60^{\circ} \mathrm{C}(2 \mathrm{~min})$, ramped to $150^{\circ} \mathrm{C}$ at $20^{\circ} \mathrm{C} / \mathrm{min}$, then to $325^{\circ} \mathrm{C}$ at $2^{\circ} \mathrm{C} / \mathrm{min}$, and held at $325^{\circ} \mathrm{C}$ for $20 \mathrm{~min}$. Quantification of individual PAH and $n$-alkanes was performed with a d14-p-terphenyl internal standard on the more sensitive Waters AutoSpec Premier mass spectrometer equipped with an Agilent 7890A gas chromatograph and using a J\&W DB-1MS coated capillary column $\left(60 \mathrm{~m} \times 0.25 \mathrm{~mm}, 0.25 \mu \mathrm{m}\right.$ film). Samples were injected in splitless mode at $320^{\circ} \mathrm{C}$ with $\mathrm{He}$ as carrier gas. The oven was programmed from $60^{\circ} \mathrm{C}$ (held for $2 \mathrm{~min}$ ) to $150^{\circ} \mathrm{C}$ at $6^{\circ} \mathrm{C} / \mathrm{min}$ then to $320^{\circ} \mathrm{C} @ 4^{\circ} \mathrm{C} / \mathrm{min}$ and held isothermal at the final temperature for 30 minutes. The source was operated in electron ionization (EI) mode at $70 \mathrm{eV}$ ionization energy at $300^{\circ} \mathrm{C}$. The AutoSpec full-scan rate was $0.80 \mathrm{~s} /$ decade over a mass range of 50 to $600 \mathrm{Da}$ and a delay of $0.20 \mathrm{~s} /$ decade. 
Metastable Reaction Monitoring-Gas Chromatography-Mass Spectrometry (MRM-GCMS) was conducted with a Waters AutoSpec Premier mass spectrometer equipped with an Agilent 7890A gas chromatograph and DB-1MS coated capillary column (60m x 0.25 $\mathrm{mm}, 0.25 \mu \mathrm{m}$ film) using splitless injection and He for carrier gas to detect polycyclic hydrocarbon biomarkers, particularly tricyclic terpanes, steranes, methylsteranes, hopanes and methylhopanes. The MRM GC temperature program used for compound separation consisted of an initial hold at $60^{\circ} \mathrm{C}$ for $2 \mathrm{~min}$, heating to $150^{\circ} \mathrm{C}$ at $10^{\circ} \mathrm{C} / \mathrm{min}$ followed by heating to $320^{\circ} \mathrm{C}$ at $3^{\circ} \mathrm{C} / \mathrm{min}$, and a final hold at $320^{\circ} \mathrm{C}$ for $22 \mathrm{~min}$. Biomarker compounds were identified based on retention time and published mass spectra and quantified in MRM GC-MS by comparison with a deuterated $\mathrm{C}_{29}$ sterane internal standard ( $d_{4}-\alpha \alpha \alpha$-24-ethylcholestane (20R), Chiron Laboratories, $\left.A S\right)$, assuming equal response factors between sample compounds and the internal standard. Individual yields of the main hopane and sterane diastereoisomers found in HyPy laboratory procedural blanks were typically $<20 \mathrm{pg}$ of total sterane and hopanes per gram of combusted silica pyrolysed, with immature $\mathrm{C}_{27} \alpha \alpha \alpha \mathrm{R}$ and/or $\mathrm{C}_{29} \alpha \alpha \alpha \mathrm{R}$ steranes being the dominant procedural blank polycyclic compounds and the hopane series usually found below detection limits. For most of the series of hopane and sterane compounds, the 'blank' reflects the detection limit of the mass spectrometer (baseline noise above which a peak must rise) rather than the identified presence of a compound in the procedural sand blank.

\section{Compound-specific stable carbon isotope analyses at MIT of HyPy products}

Compound specific carbon isotopic measurements of HyPy products were made by gas chromatography/combustion/ isotope ratio mass spectrometry (GC-C-IRMS) using a Thermo Finnigan Delta plus XP coupled to a Thermo Finnigan Trace GC at MIT. The initial oven temperature was programmed to $60^{\circ} \mathrm{C}$ (held for 2 minutes), ramped to 
$150^{\circ} \mathrm{C}$ at $6^{\circ} \mathrm{C} / \mathrm{min}$, and then to $320^{\circ} \mathrm{C}$ at $4^{\circ} \mathrm{C} / \mathrm{min}$ (held for 20.50 minutes). All samples were bracketed by pulses of in house calibrated reference $\mathrm{CO}_{2}$ gas and Oztech calibrated reference $\mathrm{CO}_{2}$ gas. A standard mix of n-alkanes (Mix A; Arndt Schimmelmann, Indiana University) was analyzed at the beginning and end of the sample sequence and after every sixth sample analysis in order to monitor the instrument condition. The root mean square (RMS) was used to compare the isotopic composition of the $15 n$-alkanes in the standard mix to their known carbon isotopic values. The RMS ranged from 0.3 to $0.1 \%$ of for the six analyses of the $n$-alkane standard mix that were performed through the sample sequence. The mean value and standard deviation of the triplicate analyses are reported here in permil (\%o) relative to Vienna Pee Dee belemnite (VPDB).

\subsubsection{Results}

Most of the results are presented and discussed in the main article, but some supporting details are discussed in the following section.

\section{Extractable hydrocarbon results}

The spikes that were used in the drilling fluid and were applied to the Teflon bags were not detected in the total lipid extracts that were analyzed in any of the laboratories. It is possible that higher spike concentrations needed to have been added to the sump water in order to be detected on sample surfaces. However, another possible explanation for the inability to detect the spikes in the rock extracts is that the water that was added to the sample bag outcompeted the spike compounds for active sites on the sample

surfaces, thereby preventing their detection and significant surficial hydrocarbon contamination in general. 
Uni Bremen found that the total sterane concentration and total hopane concentrations were up to $54.1 \mathrm{pg} / \mathrm{g}$ rock and $14.9 \mathrm{pg} / \mathrm{g}$ rock, respectively, in the combusted brick blank. These concentrations are comparable to the total hopane $(<32.9 \mathrm{pg} / \mathrm{g}$ rock) and sterane concentrations $(<37.9)$ that ANU/MIT found in their blanks (Table 1). Macquarie measured a concentration of $3.5 \mathrm{pg} / \mathrm{g}$ rock for total hopane in the procedural blank that they analyzed, which could be an underestimation of laboratory contamination due to linearity and response problems related to the standard compound class and comparatively high standard concentration. ANU/MIT and Uni Bremen quantifications were based on deuterated internal standards, $d_{4}-\mathrm{C}_{29-} \alpha \alpha \alpha-$ ethylcholestane and $d_{4-} \mathrm{C}_{27-} \alpha \alpha$ R-cholestane, which are structurally similar and had comparable concentrations to the hopane and sterane analytes of interest in the AIDP samples and blanks. In contrast, Macquarie quantified the hopanes and steranes in the AIDP samples and blanks using a deuterated PAH, $p$-terphenyl- $d_{14}$, with a total mass that was 2 orders of magnitude greater than the total used by ANU/MIT and U. Bremen (50 ng compared to $0.5 \mathrm{ng}$ ). Regardless of analysis and quantification method, every laboratory found that hopanes and steranes were often at or below detection in the AIDP samples, but if they were detectable they were comparable to the blank concentrations measured by that laboratory (see Table 1). The Macquarie team detected aliphatic hydrocarbons $\left(n-\mathrm{C}_{14-35}\right.$ alkanes, monomethylalkanes, $i-\mathrm{C}_{13-20}$ regular isoprenoids, and alkylcyclohexanes) in their samples, but the concentrations were comparable to experimental blanks for all five shale samples (0.01-1.00 $\mathrm{ng} / \mathrm{g}$ sample). Furthermore, these compounds were not detected by the ANU/MIT or Uni Bremen teams, so they are likely laboratory-specific contaminants.

All laboratories detected a suite of extractable PAHs and diamondoids. Extractable PAH and diamondoid ratios and calculated vitrinite reflectances $\left(R_{c}\right)$ are given in Tables 
S8 and S9, respectively. All laboratories found that PAHs exceeded laboratory blanks (where they were typically below detection) and had ng/g rock concentrations, where low molecular weight non-alkylated PAHs were the most abundant detected compounds in the rock extracts (Fig. S1 and S2). In general, the calculated vitrinite reflectances $\left(R_{c}\right)$ estimated from the MAI and MDI diamondoid ratios were lower than those estimated according to the MPI-1 PAH ratio (Table S9). The PAH and diamondoid ratios and $R_{c}$ values for the Jeerinah Formation sample are more variable than those calculated for the Carawine Dolomite (Table S8 and S9) because the low PAH concentrations and small peak size in the Jeerinah Formation samples affected the accuracy of peak integrations.

\section{HyPy results}

Individual PAHs, such as phenenthrene and pyrene, were detected in HyPy blanks at concentrations below $1 \mathrm{ng} / \mathrm{g}$ combusted sand, and $n$-alkanes (in the $\mathrm{C}_{14-20}$ range) had concentrations typically below $<10 \mathrm{ng} / \mathrm{g}$ sand in the sand blank. These blank concentrations are 1-2 orders of magnitude lower than the major analytes reported from Archean rock hydropyrolysates (Table S10). The 2/1/001 and 2/1/004 Carawine Dolomites generated higher yields of pyrolysate and yielded less mature and more diverse product profiles, including a series of aliphatic hydrocarbons (Figure S3) comprising a mature distribution of $\mathrm{C}_{12-20} n$-alkanes, methylalkanes, alkylcyclohexanes, diamondoids and other compound classes. The product distributions from 2/1/001 and 2/1/004 were fairly similar to those reported from HyPy of 3.4 Ga Strelley Pool chert kerogens (10) (Table S10). The PAH profiles from the pre-extracted 2.7 Ga Jeerinah Formation black shales, dominated by pyrene, phenanthrene and other unsubstitited

(parent) PAH, were similar to those generated previously for Archean black shale kerogens from the 2.5 Ga Hamersley Group of the Pilbara Craton (11). One exception was the 2/1/003 Carawine Dolomite kerogen that produced high yields of pyrolysate 
but a more mature distribution of $\mathrm{PAH}$ than the other two carbonates found stratigraphically above and below this, but this is consistent with the 2/1/003 sample containing organic matter with the lowest atomic $\mathrm{H} / \mathrm{C}$ ratio of the Carawine Dolomite samples that were pyrolysed (Table S5). This was likely due to the effects of different mineral compositions and differential heating on the extent of aromatization, thermal cracking of alkyl chains and other maturation reactions because mineral matter composition, particularly the abundance and speciation of clays, exerts a strong influence on organic matter maturation for overmature samples. Evidently, rocks containing low clay abundances preserve less metamorphosed and less recalcitrant sedimentary organic matter where lithofacies with significant carbonate or chert content attenuate the catalytic effect of acidic clay minerals in shales (10).

\subsubsection{Ratio Definitions}

Diamondoid Ratios:

$$
\begin{aligned}
& \text { MAI= 1-Methyl Adamantane/(1-Methyl Adamantane }+2-\text { Methyl Adamantane }) \\
& \text { MDI= 4-Methyl Diamantane/(1-Methyl Diamantane+3-Methyl Diamantane+4-Methyl } \\
& \quad \text { Diamantane })
\end{aligned}
$$

PAH Ratios:

$$
\begin{aligned}
& M N R=2-M e t h y l \text { Naphthalene/1-Methyl Naphthalene } \\
& M P I-1=1.5^{*}(2-M e P+3-M e P) /(P+1-M e P+9-M e P) \\
& M D F=(3-M e P+2-M e P) /(3-M e P+2-M e P+1-M e P+9-M e P)
\end{aligned}
$$

where $\mathrm{P}=$ Phenanthrene and $\mathrm{MeP}=$ Methyl Phenanthrene 


\subsubsection{SI References}

1. Buick R et al. (1995) Record of emergent continental crust $\sim 3.5$ billion years ago in the Pilbara craton of Australia. Nature 375:574-577.

2. Eigenbrode JL (2004) Late Archean Microbial Ecology: An Integration of Molecular, Isotopic, and Lithological Studies. PhD Thesis. The Pennsylvania State University, State College, PA. https://etda.libraries.psu.edu/paper/6259/.

3. Richards MN (1985) CRA Exploration Pty. Ltd. Final report on exploration licenses Ripon Hills North 45/63. Ripon Hills South 45/64 and Gingarrigan Creek 45/65, Nullagine, S F 51-5, Western Australia. Item 2655. Western Australia Geological Survey, Report number 13140, WAMEX Item Number 2655, A15932.

4. Nelson DR, Trendall AF, Altermann W (1999) Chronological correlations between the Pilbara and Kaapvaal cratons. Precambrian Res 97:165-189.

5. Woodhead JD, Hergt JM, Simonson BM (1998) Isotopic dating of an Archean bolide impact horizon, Hamersley basin, Western Australia. Geology 26:47-50.

6. Arndt NT et al. (1991) The age of the Fortescue Group, Hamersley Basin, Western Australia, from ion microprobe zircon U-Pb results. Australian Journal of Earth Sciences 38:261-281.

7. Blake TS, Buick R, Brown SJA, Barley ME (2004) Geochronology of a Late Archaean flood basalt province in the Pilbara Craton, Australia: constraints on basin evolution, volcanic and sedimentary accumulation, and continental drift rates. Precambrian Res 133:143-173.

8. Brocks JJ, Hope JM (2014) Tailing of Chromatographic Peaks in GC-MS Caused by Interaction of Halogenated Solvents with the Ion Source. J Chromatogr Sci 52:471-475.

9. Love GD, Snape CE, Carr AD, Houghton RC (1995) Release of covalently-bound alkane biomarkers in high yields from kerogen via catalytic hydropyrolysis. Org Geochem 23:981-986.

10. Marshall CP et al. (2007) Structural characterization of kerogen in 3.4 Ga Archaean cherts from the Pilbara Craton, Western Australia. Precambrian Res 155:1-23.

11. Brocks JJ et al. (2003) Release of bound aromatic hydrocarbons from late Archean and Mesoproterozoic kerogens via hydropyrolysis. Geochim Cosmochim Acta 67:1521-1530.

12. Boreham CJ, Crick IH, Powell TG (1988) Alternative calibration of the Methylphenanthrene Index against vitrinite reflectance: Application to maturity measurements on oils and sediments. Org Geochem 12:289-294.

13. Chen J, Fu J, Sheng G, Liu D, Zhang J (1996) Diamondoid hydrocarbon ratios: novel maturity indices for highly mature crude oils. Org Geochem 25:179-190. 


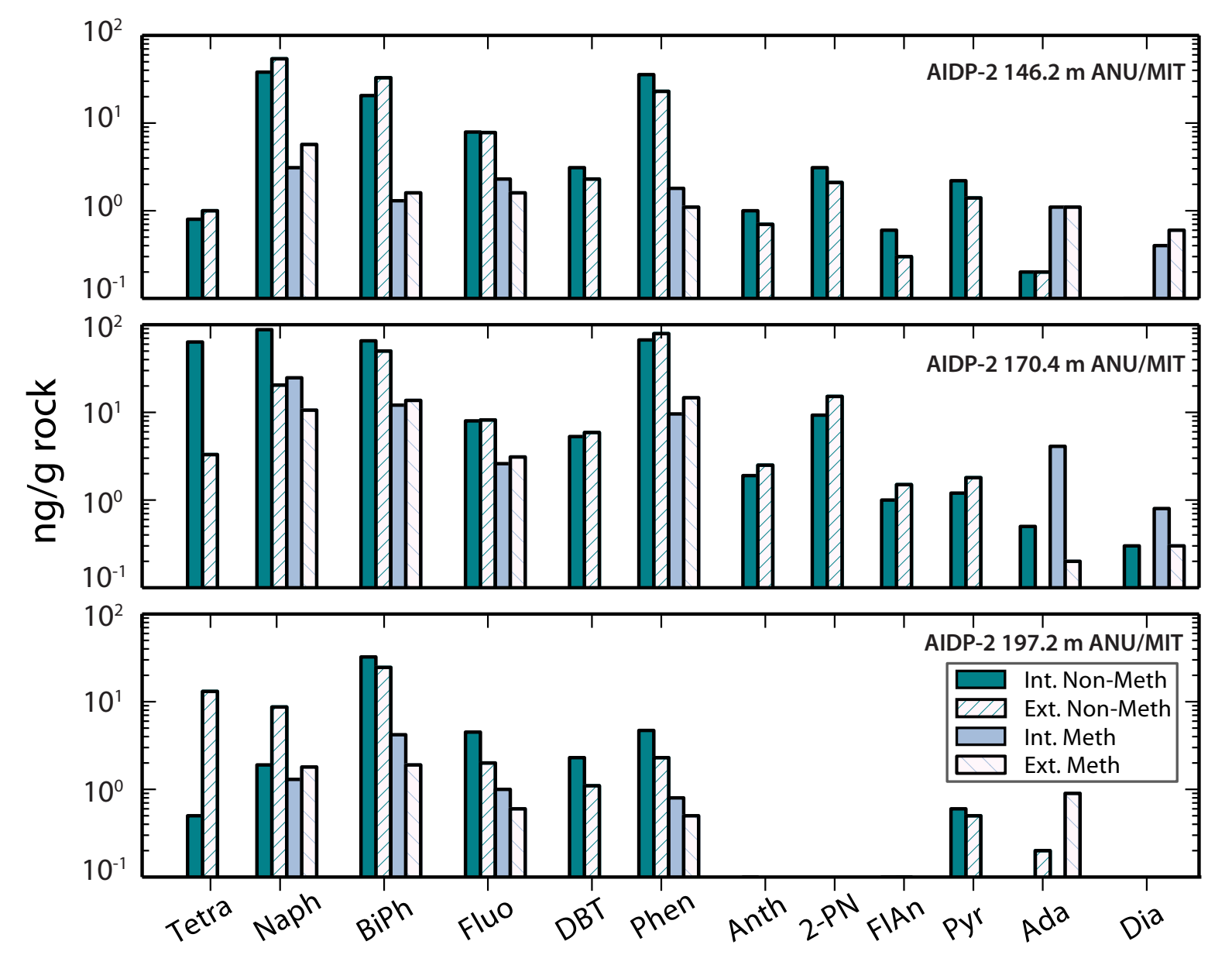

Figure S1. PAH and diamondoid quantification for ANU/MIT Laboratory Analysis. The interior and exteriors show similar concentrations. Non-methylated PAHs and diamondoids are more abundant than their corresponding methylated compounds. Low molecular weight PAHs are more abundant compared to higher molecular weight PAHs. Compounds are abbreviated accordingly: Tetra=tetralin; Naph=naphthalene; BiPh=biphenyl; Fluo=fluorene; $\quad$ DBT=dibenzothiophene; Phen=phenanthrene; Anth=anthracene; 2-PN=2-phenylnaphthalene; FlAn=fluoranthene; Pyr=pyrene, $\mathrm{Ada}=$ adamantane; and Dia=diamantane. 

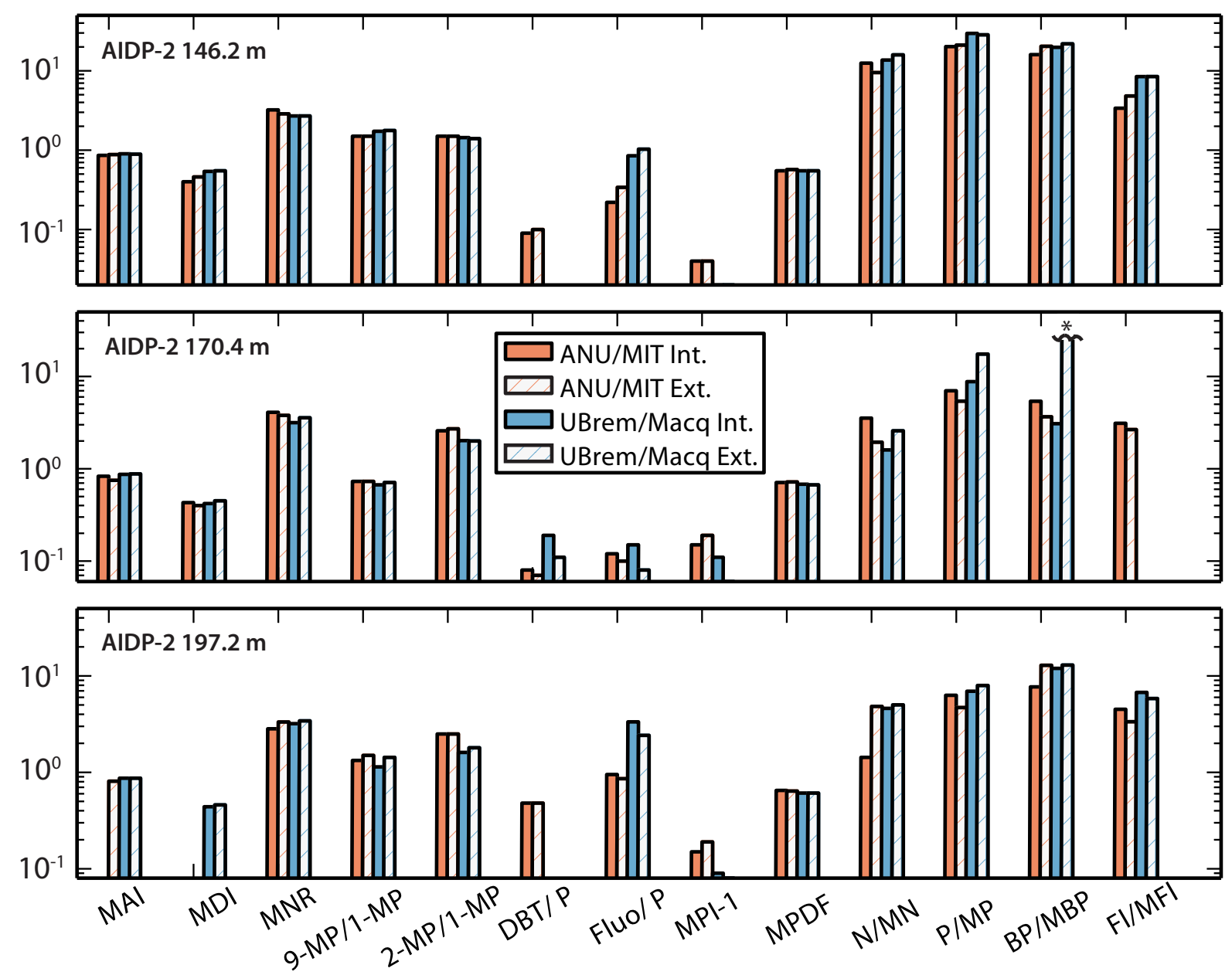

Fig. S2. Multi-Laboratory Reproducibility of PAH and Diamondoid Ratios. Molecular ratios compare well between laboratories, both for core interior and exterior sample pieces. The column noted with an asterisk was truncated due to an anomalously high value of 203 that put it off the scale. Ratios are defined in the SI and the abbreviations are as follows: MAI=Methyl Adamantane Index, MDI=Methyl Diamantane Index; MNR=Methyl Naphthalene Ratio; 9-MP/1-MP=9-Methyl Phenanthrene/1-Methyl Phenanthrene; 2-MP/1-MP=2-Methyl Phenanthrene/1-Methyl Phenanthrene; DBT/P= Dibenzothiophene/Phenanthrene; $\quad$ Fluo/P=Fluorene/Phenenthrene; $\quad$ MPI1=Methylphenanthrene Index; $\mathrm{MPDF}=$ Methylphenanthrene distribution factor; N/MN= Naphthalene/Methyl Naphthalenes; $\mathrm{P} / \mathrm{MP}=$ Phenanthrene/Methyl Phenanthrenes; $\mathrm{BP} / \mathrm{MBP}=$ Biphenyl/Methyl Biphenyls; Fluorene/Methyl Fluorenes. 


\section{AIDP-2 116.09 m HyPy Saturate Fraction Full Scan (UCR)}

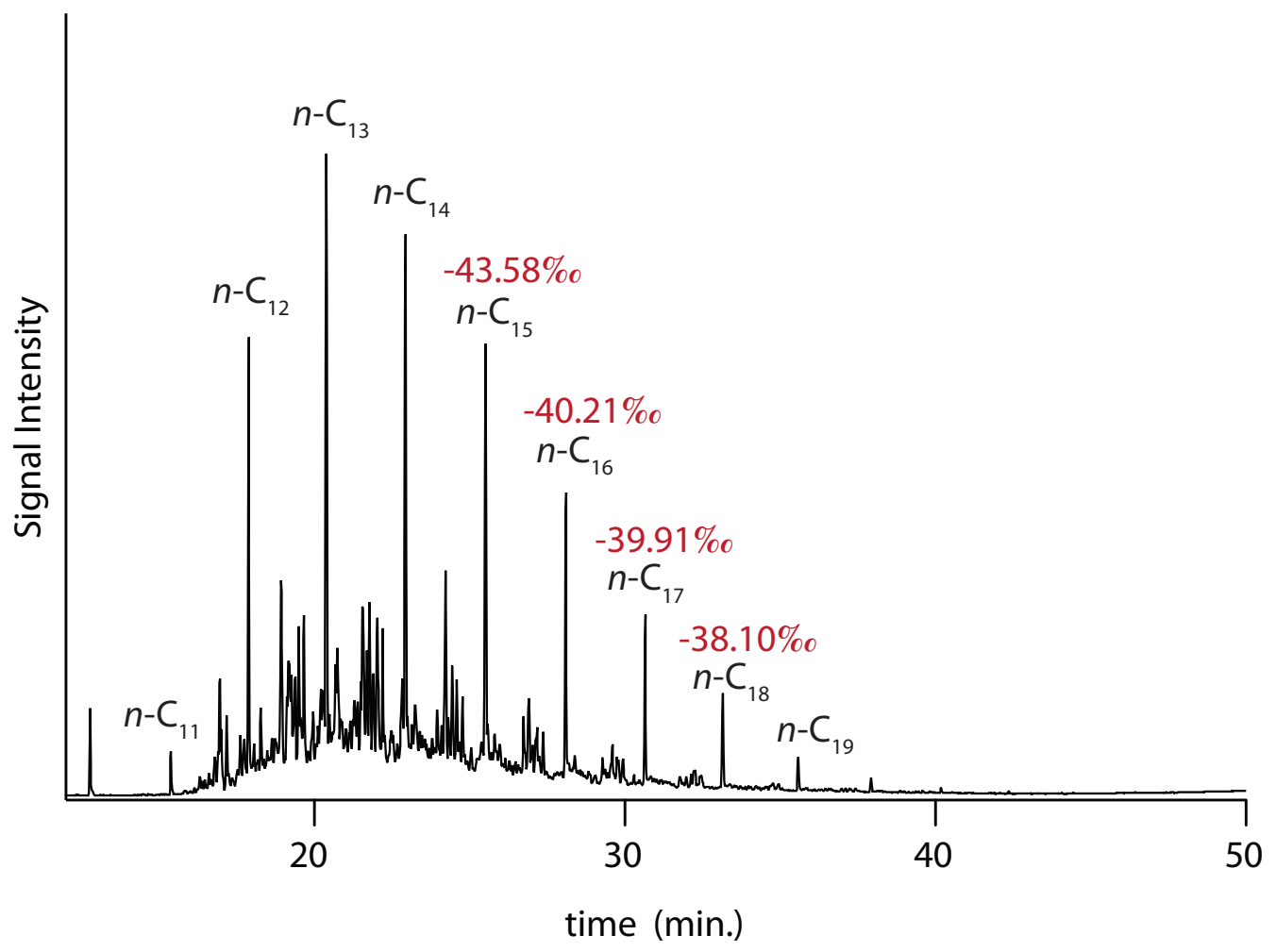

Fig S3. HyPy total ion current (TIC) chromatogram of AIDP-2/1/001-116.09m aliphatic hydrocarbons generated from the kerogen concentrate. The $n$-alkanes are labeled for reference, and the $\delta^{13} \mathrm{C}$ values for $\mathrm{C}_{15}-\mathrm{C}_{18} n$-alkanes for this sample, which are reported in Table S7, are denoted in red. Other abundant alkane compound series detected include methylalkanes, alkylcyclohexanes and diamondoids. 
Table S1. ANU/MIT sample description and distribution

\begin{tabular}{|c|c|c|c|c|c|c|c|}
\hline Sample ID & Depth & Hole & Formation & Lithology & $\begin{array}{c}\text { Extractable } \\
\text { Biomarker } \\
\text { Laboratory }\end{array}$ & $\begin{array}{c}\text { Extractable } \\
\text { Biomarker } \\
\text { Laboratory }\end{array}$ & $\begin{array}{l}\text { UCR } \\
\text { HyPy }\end{array}$ \\
\hline $1 / 1 / 004$ & 186.00 & AIDP-1 & Coucal Fm. & $\begin{array}{l}\text { Massive chlorite-plagioclase-actinolite } \\
\text { metadolerite (negative control) }\end{array}$ & Macq. Uni & & \\
\hline $2 / 1 / 001$ & 116.09 & AIDP-2 & Carawine Fm. & $\begin{array}{l}\text { Wavey-laminated grey-black fine-grained } \\
\text { dolarenite with pyrobituminous stylolites }\end{array}$ & U. Bremen & & Yes \\
\hline $2 / 1 / 002$ & 133.55 & AIDP-2 & Carawine Fm. & pyrobitumen styolite & $\begin{array}{l}\text { ANU/U. } \\
\text { Bremen }\end{array}$ & & \\
\hline $2 / 1 / 003$ & 146.20 & AIDP-2 & Carawine Fm. & $\begin{array}{l}\text { Interlayered fine-grained dolarenite and black } \\
\text { kerogenous dolosiltite }\end{array}$ & ANU/MIT & Macq. Uni & Yes \\
\hline $2 / 1 / 004$ & 170.40 & AIDP-2 & Carawine Fm. & Interlayered coarse- and fine-grained dolarenite & ANU/MIT & U. Bremen & Yes \\
\hline $2 / 1 / 005$ & 197.20 & AIDP-2 & Carawine Fm. & $\begin{array}{l}\text { Interlayered fine-grained dolarenite and black } \\
\text { kerogenous dolosiltite }\end{array}$ & ANU/MIT & Macq. Uni & \\
\hline $2 / 1 / 007$ & 291.28 & AIDP-2 & Jeerinah Fm. & $\begin{array}{l}\text { Plane-laminated black kerogenous marly } \\
\text { mudstone with } 1 \% \text { disseminated pyrite }\end{array}$ & ANU/MIT & $\begin{array}{l}\text { Macq. Uni, } \\
\text { U. Bremen }\end{array}$ & Yes \\
\hline $2 / 1 / 008$ & 301.40 & AIDP-2 & Jeerinah Fm. & $\begin{array}{l}\text { Massive black kerogenous shale with } 1 \% \\
\text { disseminated pyrite }\end{array}$ & ANU/MIT & U. Bremen & \\
\hline $2 / 1 / 010$ & 322.82 & AIDP-2 & Jeerinah Fm. & $\begin{array}{l}\text { Massive black kerogenous shale with } 5 \% \text { pyrite } \\
\text { nodules }\end{array}$ & U. Bremen & & Yes \\
\hline $2 / 1 / 011$ & 327.42 & AIDP-2 & Jeerinah Fm. & $\begin{array}{l}\text { Massive black kerogenous shale with } 5 \% \text { pyrite } \\
\text { nodules }\end{array}$ & ANU/MIT & Macq. Uni & Yes \\
\hline $2 / 1 / 012$ & 325.48 & AIDP-2 & Jeerinah Fm. & $\begin{array}{l}\text { Massive black kerogenous shale with } 5 \% \text { pyrite } \\
\text { nodules }\end{array}$ & ANU/MIT & & \\
\hline $2 / 1 / 014$ & 349.00 & AIDP-2 & Jeerinah Fm. & chert (negative control) & U. Bremen & & \\
\hline $2 / 1 / 016$ & 441.00 & AIDP-2 & Jeerinah Fm. & $\begin{array}{l}\text { Massive basalt with } 1 \% \text { amygdales (negative } \\
\text { control) }\end{array}$ & ANU/MIT & & \\
\hline $3 / 1 / 004$ & 98.36 & AIDP-3 & Jeerinah Fm. & $\begin{array}{l}\text { Massive black kerogenous mudstone and grey } \\
\text { chert }\end{array}$ & ANU/MIT & & Yes \\
\hline $3 / 1 / 005$ & 130.30 & AIDP-3 & Jeerinah Fm. & $\begin{array}{l}\text { Massive black kerogenous mudstone with } 1 \% \\
\text { pyrite nodules }\end{array}$ & ANU/MIT & Macq. Uni & Yes \\
\hline
\end{tabular}


Table S2. ANU/MIT sample preparation

\begin{tabular}{|c|c|c|c|c|c|}
\hline & Sample ID & Depth (m) & $\begin{array}{l}\text { Blank } \\
\text { Extracted (g) }\end{array}$ & $\begin{array}{l}\text { Extracted } \\
\text { Interior (g) }\end{array}$ & $\begin{array}{l}\text { Mass } \\
\text { Exterior (g) }\end{array}$ \\
\hline \multicolumn{6}{|l|}{ Round 1} \\
\hline & Brick Blank Nov. & $n / a$ & 24.3 & $n / a$ & $n / a$ \\
\hline & $2 / 1 / 016$ & 441.00 & 24.7 & 28.1 & 14.4 \\
\hline & $2 / 1 / 007$ & 291.28 & 14.9 & 36.9 & 25.9 \\
\hline & $2 / 1 / 003$ & 146.2 & 17.0 & 19.1 & 19.8 \\
\hline & $3 / 1 / 005$ & 130.30 & $n / a$ & 19.8 & 12.3 \\
\hline \multicolumn{6}{|l|}{ Round 2} \\
\hline & Brick blank Jan. & $n / a$ & 27.7 & $n / a$ & $n / a$ \\
\hline & $3 / 1 / 004$ & 98.36 & 11.2 & 25.3 & 17.8 \\
\hline & $2 / 1 / 008$ & 301.40 & 10.0 & 25.5 & 13.0 \\
\hline & $2 / 1 / 011$ & 327.42 & 11.1 & 22.3 & 15.5 \\
\hline & $2 / 1 / 005$ & 197.20 & 6.3 & 29.7 & 23.1 \\
\hline & RHDH2A Sand blank & $130.2-130.4^{\mathrm{a}}$ & 17.11 & 31.12 & \\
\hline & RHDH2A cut surface 1 & $130.2-130.4^{\mathrm{a}}$ & & & 25.38 \\
\hline & RHDH2A cut surface 2 & $130.2-130.4^{\mathrm{a}}$ & & & 29.37 \\
\hline & $\mathrm{RHDH} 2 \mathrm{~A}$ rounded & $130.2-130.4^{\mathrm{a}}$ & & & 31.24 \\
\hline \multicolumn{6}{|l|}{ Round 3} \\
\hline & Brick blank July & $n / a$ & 21.64 & $n / a$ & $n / a$ \\
\hline & $2 / 1 / 012$ & 325.48 & 6.69 & 23.0 & 24.5 \\
\hline & $2 / 1 / 004$ & 170.40 & 6.32 & 30.7 & 28.9 \\
\hline
\end{tabular}


Table S3. U. Bremen Sample Preparation

\begin{tabular}{llll}
\hline Sample ID & Depth (m) & $\begin{array}{l}\text { Extracted } \\
\text { exterior }(\mathbf{g})\end{array}$ & $\begin{array}{l}\text { Extracted } \\
\text { interior }(\mathbf{g})\end{array}$ \\
\hline Brick blank & $n / a$ & 8.17 & 8.17 \\
$2 / 1 / 014$ & 349.00 & 8.11 & 8.06 \\
$2 / 1 / 010$ & 322.82 & 8.23 & 8.04 \\
$2 / 1 / 008$ & 301.40 & 8.10 & 8.35 \\
$2 / 1 / 007$ & 291.28 & 8.04 & 8.11 \\
$2 / 1 / 004$ & 170.40 & 8.32 & 8.31 \\
$2 / 1 / 001$ & 116.09 & 8.34 & 8.33 \\
\hline
\end{tabular}

Table S4. Macquarie Sample Preparation

\begin{tabular}{llll}
\hline Sample ID & Depth $(\mathbf{m})$ & $\begin{array}{l}\text { Extracted } \\
\text { exterior }(\mathbf{g})\end{array}$ & $\begin{array}{l}\text { Extracted } \\
\text { interior }(\mathbf{g})\end{array}$ \\
\hline $1 / 1 / 004$ & 186.00 & 61.8 & 28.3 \\
$3 / 1 / 005$ & 130.30 & 34.5 & 51.7 \\
$2 / 1 / 003$ & 146.20 & 51.4 & 40.4 \\
$2 / 1 / 005$ & 197.20 & 131.5 & 62.7 \\
$2 / 1 / 011$ & 327.42 & 49.2 & 23.2 \\
$2 / 1 / 007$ & 291.28 & 72.4 & 123.5 \\
\hline
\end{tabular}


Table S5. TOC, bulk organic $\delta^{13} \mathrm{C}$, and $\mathrm{H} / \mathrm{C}$

\begin{tabular}{|c|c|c|c|c|c|c|c|}
\hline $\begin{array}{l}\text { Sample } \\
\text { ID }\end{array}$ & $\begin{array}{l}\text { TOC (\%) } \\
\text { ANU/MIT }\end{array}$ & $\begin{array}{l}\text { Average } \\
\delta^{13} \mathbf{C}_{\text {org }}(\%) \\
\text { ANU/MIT }^{\text {a }}\end{array}$ & $\begin{array}{l}\text { Average } \\
\delta^{13} C_{\text {org }}(\%) \\
U^{\text {Bremen }}{ }^{\text {a }}\end{array}$ & $\begin{array}{l}\text { Carbonate } \\
\text { Content } \\
(\%) \\
\text { ANU/MIT }\end{array}$ & $\begin{array}{l}\text { Carbonate } \\
\text { Content } \\
(\%) \text { U. } \\
\text { Bremen }\end{array}$ & $\begin{array}{l}\text { Silicate } \\
\text { Content } \\
(\%) \text { U. } \\
\text { Bremen }\end{array}$ & $\begin{array}{l}\text { H/C U. } \\
\text { Bremen }^{\mathrm{d}}\end{array}$ \\
\hline $2 / 1 / 001$ & $0.4^{\mathrm{e}}$ & & -43.1 & & 90.7 & 7.6 & 0.54 \\
\hline $2 / 1 / 002$ & $3.2^{\mathrm{f}}$ & & $-32.4^{\mathrm{f}}$ & & & & \\
\hline $2 / 1 / 003$ & 1.6 & $-43.1 \pm 0.2$ & -43.3 & 69.4 & 67.8 & 11.0 & 0.24 \\
\hline $2 / 1 / 004$ & 1.3 & $-45.8 \pm 0.6$ & -47.1 & 78.7 & 81.9 & 14.7 & 0.46 \\
\hline $2 / 1 / 005$ & 1.5 & $-49.7 \pm 0.3$ & -49.9 & 40.9 & 29.1 & 6.8 & 0.35 \\
\hline $2 / 1 / 007$ & 6.7 & $-48.1 \pm 0.7$ & -48.8 & 11.0 & 12.8 & 28.6 & 0.23 \\
\hline $2 / 1 / 008^{\mathrm{g}}$ & 5.4 & -42.1 & -42.2 & 11.6 & 11.3 & 15.6 & 0.4 \\
\hline 2/1/010 & $5.8^{\mathrm{e}}$ & & -43.6 & & 4.9 & 38.1 & 0.19 \\
\hline 2/1/011 & 4.2 & $-43.6 \pm 0.7$ & -44.4 & 2.0 & 8.8 & 42.8 & 0.24 \\
\hline $2 / 1 / 012$ & 3.7 & $-44.5 \pm 0.2$ & -44.8 & 3.1 & 6.3 & 23.8 & 0.25 \\
\hline 3/1/004 & 2.8 & -41.7 & -42.0 & 2.1 & 5.3 & $n / a$ & 0.44 \\
\hline 3/1/005 & 3.4 & $-41.4 \pm 0.1$ & -41.9 & 3.5 & 12.8 & 28.9 & 0.24 \\
\hline
\end{tabular}

$\overline{{ }^{a} \text { Reported against VPD } B}$

${ }^{\mathrm{b}}$ Loss upon digestion with $\mathrm{HCl}$

${ }^{\mathrm{c}}$ Loss upon digestion with $\mathrm{HF}$

${ }^{\mathrm{d}}$ Atomic $\mathrm{H} / \mathrm{C}$ ratios are calculated from the elemental composition as $(11.92 * \% \mathrm{H}) / \% \mathrm{C}$

${ }^{\mathrm{e}}$ TOC measurements made on 2/1/001 and 2/1/010 interior rock powders from UCR were analyzed by standard methods using a LECO C230 instrument at GeoMark Research

${ }^{\mathrm{f}}$ Measured at ANU by Uni Bremen

${ }^{g}$ 2/1/8 was not measured in duplicate due to an autosampler error 
Table S6. Compound Specific $\delta^{13} \mathrm{C}$ Analysis of extractable PAHs ${ }^{\mathrm{a}}$

\begin{tabular}{|c|c|c|c|c|c|c|c|c|c|c|}
\hline \multirow[t]{2}{*}{ PAH } & \multirow{2}{*}{$\begin{array}{c}\text { 2/1/001 } \\
\text { U. Bremen } \\
\delta^{13} C\end{array}$} & \multirow{2}{*}{$\begin{array}{c}2 / 1 / 002 \\
\text { ANU/U. Bremen } \\
\delta^{13} C\end{array}$} & \multicolumn{2}{|c|}{$\begin{array}{c}2 / 1 / 003 \\
\text { ANU/MIT }\end{array}$} & \multicolumn{2}{|c|}{$\begin{array}{c}2 / 1 / 004 \\
\text { ANU/MIT }\end{array}$} & \multicolumn{2}{|c|}{$\begin{array}{c}\text { 2/1/004 } \\
\text { U. Bremen }\end{array}$} & \multicolumn{2}{|c|}{$\begin{array}{c}\text { 2/1/005 } \\
\text { ANU/MIT }\end{array}$} \\
\hline & & & $\delta^{13} \mathrm{C}$ & $s d$ & $\delta^{13} C$ & $s d$ & $\delta^{13} \mathrm{C}$ & $s d$ & $\delta^{13} C$ & $s d$ \\
\hline Tetralin & & & & & -30.3 & 0.1 & & & & \\
\hline Naphthalene & & & & & -30.0 & 0.1 & & & & \\
\hline Methyltetrahydronaphthalene & & & & & -31.2 & 0.1 & & & & \\
\hline $\begin{array}{l}\text { Methyltetrahydronaphthalene }+ \\
\text { 2-methylnaphthalene }\end{array}$ & & & & & -30.2 & 0.1 & & & & \\
\hline 1-methylnaphthalene & & & & & -29.3 & 0.1 & & & & \\
\hline Biphenyl & & -33.1 & & & -33.8 & 0.1 & -33.3 & 0.1 & -34.3 & 0.4 \\
\hline 3-Methylbiphenyl & & -31.2 & & & -33.3 & 0.2 & -32.2 & 0.5 & & \\
\hline 4-Methylbiphenyl & & & & & -33.4 & 0.2 & & & & \\
\hline Fluorene & & -32.3 & & & & & -29.7 & 1.4 & -26.0 & 0.2 \\
\hline $\begin{array}{l}\text { Octahydrophenanthrene/ } \\
\text { Octahydroanthracene }\end{array}$ & & & & & & & -30.3 & 1.4 & & \\
\hline $\begin{array}{l}\text { Fluorene+ } \\
\text { Octahydroanthracene/ } \\
\text { Octahydrophenanthrene }\end{array}$ & & & & & -33.7 & 0.3 & & & & \\
\hline $\begin{array}{l}\text { Tetrahydrophenanthrene/ } \\
\text { Tetrahydroanthracene + DBT }\end{array}$ & & & & & -30.8 & 0.3 & -27.5 & 0.1 & & \\
\hline Phenanthrene & -36.9 & -32.6 & -34.2 & 0.1 & -35.5 & 0.1 & -35.6 & 0.1 & -36.2 & 0.1 \\
\hline $\begin{array}{l}\text { Methyltetrahydrophenanthrene/ } \\
\text { methyltetrahydroanthracene }\end{array}$ & & & & & & & -26.5 & & & \\
\hline $\begin{array}{l}\text { Methyltetrahydrophenanthrene/ } \\
\text { methyltetrahydroanthracene }\end{array}$ & & & & & -29.6 & 0.2 & & & & \\
\hline Phenyltetralin & & & & & -33.6 & 0.1 & -33.6 & 0.2 & & \\
\hline 2-Phenylnaphthalene & -33.9 & -33.2 & & & -32.8 & 0.1 & -31.7 & 0.9 & & \\
\hline
\end{tabular}




\begin{tabular}{|c|c|c|c|c|}
\hline Tetrahydrobenzanthracene & & -34.0 & 0.1 & -32.2 \\
\hline Fluoranthene & -33.4 & & & \\
\hline Pyrene & -36.4 & & & \\
\hline p-Terphenyl & -35.2 & & & \\
\hline Benzo[a]pyrene & -30.1 & & & \\
\hline 242 Da unidentified PAH & -31.7 & & & \\
\hline Benzo-Naphtho-Thiophene & -41.3 & & & \\
\hline Benz[a]anthracene & -40.5 & & & \\
\hline Benz[k]fluoranthene & -38.9 & & & \\
\hline
\end{tabular}

${ }^{\mathrm{a}}$ All values reported in \%o in reference to $V P D B$

Table S7. Compound Specific $\delta^{13} \mathrm{C}$ Analysis of HyPy products generated from kerogens/extracted rocks ${ }^{a}$

\begin{tabular}{|c|c|c|c|c|c|c|c|c|c|c|}
\hline & \multicolumn{2}{|c|}{$2 / 1 / 001$} & \multicolumn{2}{|c|}{$2 / 1 / 003$} & \multicolumn{2}{|c|}{$2 / 1 / 004^{b}$} & \multicolumn{2}{|c|}{$2 / 1 / 007$} & \multicolumn{2}{|c|}{$2 / 1 / 011$} \\
\hline & $\delta^{13} \mathrm{C}$ & $s d$ & $\delta^{13} C$ & sd & $\delta^{13} C$ & $s d$ & $\delta^{13} \mathrm{C}$ & $s d$ & $\delta^{13} C$ & $s d$ \\
\hline Dihydropyrene (204 Da)+ Fluoranthene (202 Da) & & & -43.7 & 0.7 & & & -45.9 & 1.0 & -43.2 & 0.9 \\
\hline Pyrene & -43.6 & 0.5 & -43.7 & 0.2 & -45.4 & 0.6 & -45.3 & 0.8 & -42.6 & 0.5 \\
\hline Me-Pyrene & -43.1 & 1.1 & & & -45.1 & 0.5 & & & & \\
\hline Unidentified $242 \mathrm{Da}$ PAH & -44.9 & 1.0 & & & -45.2 & 0.1 & & & & \\
\hline Benzo(ghi)perylene & -44.4 & 0.2 & & & & & & & & \\
\hline $\mathrm{C}_{15} n$-alkane & -43.6 & 0.2 & & & & & & & & \\
\hline $\mathrm{C}_{16} n$-alkane & -40.2 & 0.3 & & & & & & & & \\
\hline $\mathrm{C}_{17} n$-alkane & -39.9 & 0.8 & & & & & & & & \\
\hline $\mathrm{C}_{18} n$-alkane & -38.1 & 0.2 & & & & & & & & \\
\hline
\end{tabular}

${ }^{\mathrm{a}}$ All values reported in $\%$ in reference to $V P D B$

${ }^{b}$ Based on duplicate measurements due to an analytical error in the triplicate measurement for this sample 
Table S8. Extractable PAH \& Diamondoid Ratios

\begin{tabular}{|c|c|c|c|c|c|c|c|c|c|c|c|c|c|c|c|}
\hline Sample & $\begin{array}{l}\text { Int./ } \\
\text { Ext. }\end{array}$ & Lab. & $\begin{array}{c}\text { MPI- } \\
1 \\
\end{array}$ & MPDF & MAI & MDI & DBT/P & Fluo/P & $\mathbf{P} / \mathbf{M P}$ & $\begin{array}{l}\text { 9-MP/ } \\
\text { 1-MP }\end{array}$ & $\begin{array}{l}\text { 2-MP/ } \\
1-\mathrm{MP}\end{array}$ & $\mathbf{N} / \mathbf{M N}$ & MNR & BP/MBP & Fl/MFl \\
\hline \multirow[t]{2}{*}{$2 / 1 / 001$} & Int. & U. Bremen & 0.01 & 0.49 & 0.94 & 0.59 & 0.51 & 0.00 & 81.9 & 1.52 & 1.29 & 25.0 & 2.34 & 64.1 & \\
\hline & Ext. & U. Bremen & 0.01 & 0.49 & 0.92 & 0.62 & 0.55 & 0.00 & 64.2 & 1.53 & 1.30 & 15.5 & 1.98 & 77.6 & \\
\hline $2 / 1 / 002$ & $\mathrm{n} / \mathrm{a}$ & ANU/U. Br & 0.09 & 0.66 & 0.89 & 0.60 & 0.12 & 0.31 & 10.3 & 1.91 & 3.00 & 3.38 & 3.18 & 6.46 & 7.80 \\
\hline \multirow[t]{4}{*}{$2 / 1 / 003$} & Int. & ANU/MIT & 0.04 & 0.55 & 0.86 & 0.40 & 0.09 & 0.22 & 20.2 & 1.50 & 1.50 & 12.5 & 3.22 & 16.0 & 3.38 \\
\hline & Ext. & ANU/MIT & 0.04 & 0.57 & 0.88 & 0.46 & 0.10 & 0.34 & 21.1 & 1.50 & 1.50 & 9.51 & 2.87 & 20.4 & 4.82 \\
\hline & Int. & Macq. U & 0.02 & 0.55 & 0.90 & 0.54 & & 0.85 & 29.6 & 1.73 & 1.44 & 13.7 & 2.70 & 19.8 & 8.46 \\
\hline & Ext. & Macq. U & 0.02 & 0.55 & 0.89 & 0.55 & & 1.03 & 28.4 & 1.77 & 1.40 & 15.9 & 2.70 & 21.9 & 8.47 \\
\hline \multirow[t]{4}{*}{$2 / 1 / 004$} & Int. & U. Bremen & 0.11 & 0.68 & 0.87 & 0.42 & 0.19 & 0.15 & 8.79 & 0.67 & 2.02 & 1.60 & 3.16 & 3.08 & \\
\hline & Ext. & U. Bremen & 0.06 & 0.67 & 0.88 & 0.45 & 0.11 & 0.08 & 17.5 & 0.71 & 2.00 & 2.58 & 3.57 & 203 & \\
\hline & Int. & ANU/MIT & 0.15 & 0.71 & 0.83 & 0.43 & 0.08 & 0.12 & 7.00 & 0.73 & 2.58 & 3.54 & 4.09 & 5.39 & 3.11 \\
\hline & Ext. & ANU/MIT & 0.19 & 0.72 & 0.75 & 0.40 & 0.07 & 0.10 & 5.40 & 0.73 & 2.71 & 1.94 & 3.79 & 3.66 & 2.66 \\
\hline \multirow[t]{4}{*}{$2 / 1 / 005$} & Int. & ANU/MIT & 0.15 & 0.65 & na & na & 0.48 & 0.95 & 6.30 & 1.33 & 2.50 & 1.43 & 2.83 & 7.70 & 4.51 \\
\hline & Ext. & ANU/MIT & 0.19 & 0.64 & 0.81 & na & 0.48 & 0.86 & 4.70 & 1.50 & 2.50 & 4.83 & 3.33 & 12.9 & 3.35 \\
\hline & Int. & Macq. U & 0.09 & 0.61 & 0.87 & 0.44 & & 3.34 & 6.94 & 1.14 & 1.61 & 4.61 & 3.20 & 12.0 & 6.75 \\
\hline & Ext. & Macq. U & 0.08 & 0.61 & 0.87 & 0.46 & & 2.42 & 7.96 & 1.43 & 1.80 & 5.01 & 3.42 & 13.0 & 5.83 \\
\hline \multirow[t]{4}{*}{$2 / 1 / 007$} & Int. & U. Bremen & 0.21 & 0.74 & $n a$ & $n a$ & 0.45 & 2.86 & 4.99 & 1.15 & 2.59 & 1.23 & 6.55 & 2.76 & \\
\hline & Ext. & U. Bremen & 0.07 & 1.00 & $n a$ & 0.09 & 0.45 & 1.86 & 21.1 & zero & zero & 1.28 & 6.22 & 3.53 & \\
\hline & Int. & Macq. U & 0.68 & 0.63 & 0.77 & 0.49 & & 4.45 & 0.72 & 1.04 & 1.77 & 1.02 & 5.96 & 1.27 & 1.43 \\
\hline & Ext. & Macq. U & & & 1.00 & 0.39 & & 3.99 & & & & 0.98 & 7.22 & 1.40 & 1.41 \\
\hline \multirow[t]{4}{*}{$2 / 1 / 008$} & Int. & U. Bremen & 0.22 & 0.69 & $n a$ & 0.04 & 0.49 & 2.46 & 4.34 & 1.11 & 2.05 & 1.53 & 5.23 & 4.01 & \\
\hline & Ext. & U. Bremen & 0.26 & 0.71 & na & 0.11 & 4.11 & 1.66 & 3.88 & 1.14 & 2.32 & 1.14 & 5.83 & 2.71 & \\
\hline & Int. & Macq. U & & & & & 0.40 & 1.10 & & & & 0.71 & 10.4 & & \\
\hline & Ext. & Macq. U & & & & & 0.33 & 0.83 & & & & 0.75 & 8.00 & & \\
\hline \multirow[t]{2}{*}{$2 / 1 / 010$} & Int. & U. Bremen & 0.22 & 0.73 & 0.81 & 0.05 & 0.58 & 1.46 & 4.66 & 1.13 & 2.57 & 1.89 & 5.18 & 3.80 & \\
\hline & Ext. & U. Bremen & 0.01 & 0.64 & & & 0.38 & 0.05 & 134 & 1.27 & 1.84 & 1.22 & 5.52 & 18.6 & \\
\hline \multirow[t]{4}{*}{$2 / 1 / 011$} & Int. & ANU/MIT & & & & & 0.43 & 1.14 & & & & 9.75 & 4.00 & & \\
\hline & Ext. & ANU/MIT & & & & & 0.43 & 1.00 & & & & 9.40 & 1.50 & & \\
\hline & Int. & U. Bremen & 0.28 & 0.59 & 0.76 & 0.62 & & 9.78 & 1.88 & 1.10 & 1.37 & 0.82 & 6.52 & 1.51 & 2.09 \\
\hline & Ext. & U. Bremen & & & & & & & & & & & & & \\
\hline
\end{tabular}


Table S9. Calculated Vitrinite Reflectance (\% $\left.R_{c}\right)$

\begin{tabular}{|c|c|c|c|c|c|}
\hline Sample & $\begin{array}{l}\text { Interior/ } \\
\text { Exterior }\end{array}$ & Lab. & $\mathrm{R}_{\mathrm{c}}(\mathrm{MPI}-1)^{\mathrm{a}}$ & $\mathbf{R}_{\mathrm{c}}(\mathrm{MAI})^{\mathrm{b}}$ & $R_{c}(M D I){ }^{c}$ \\
\hline \multirow[t]{2}{*}{$2 / 1 / 001$} & Int. & U.Bremen & 3.00 & 1.98 & 1.84 \\
\hline & Ext. & U.Bremen & 2.99 & 1.94 & 1.91 \\
\hline $2 / 1 / 002$ & $\mathrm{n} / \mathrm{a}$ & $\begin{array}{l}\text { ANU/ } \\
\text { U.Bremen }\end{array}$ & 2.95 & 1.88 & 1.86 \\
\hline \multirow[t]{4}{*}{ 2/1/003 } & Int. & ANU/MIT & 2.98 & 1.82 & 1.40 \\
\hline & Ext. & ANU/MIT & 2.98 & 1.86 & 1.54 \\
\hline & Int. & Macq. U & 2.99 & 1.90 & 1.72 \\
\hline & Ext. & Macq. U & 2.99 & 1.88 & 1.75 \\
\hline \multirow[t]{4}{*}{ 2/1/004 } & Int. & U.Bremen & 2.94 & 1.85 & 1.45 \\
\hline & Ext. & U.Bremen & 2.97 & 1.86 & 1.51 \\
\hline & Int. & ANU/MIT & 2.92 & 1.76 & 1.47 \\
\hline & Ext. & ANU/MIT & 2.90 & 1.60 & 1.40 \\
\hline \multirow[t]{4}{*}{ 2/1/005 } & Int. & ANU/MIT & 2.92 & & \\
\hline & Ext. & ANU/MIT & 2.90 & 1.72 & \\
\hline & Int. & Macq. U & 2.95 & 1.84 & 1.49 \\
\hline & Ext. & Macq. U & 2.96 & 1.84 & 1.54 \\
\hline \multirow[t]{4}{*}{ 2/1/007 } & Int. & U.Bremen & 2.88 & & \\
\hline & Ext. & U.Bremen & 2.96 & & 0.69 \\
\hline & Int. & Macq. U & 2.63 & 1.64 & 1.61 \\
\hline & Ext. & Macq. U & & 2.10 & 1.38 \\
\hline \multirow[t]{4}{*}{ 2/1/008 } & Int. & U.Bremen & 2.88 & & 0.59 \\
\hline & Ext. & U.Bremen & 2.86 & & 0.75 \\
\hline & Int. & Macq. U & & & \\
\hline & Ext. & Macq. U & & & \\
\hline \multirow[t]{2}{*}{ 2/1/010 } & Int. & U.Bremen & 2.88 & 1.72 & 0.61 \\
\hline & Ext. & U.Bremen & 3.00 & & \\
\hline \multirow[t]{4}{*}{ 2/1/011 } & Int. & ANU/MIT & & & \\
\hline & Ext. & ANU/MIT & & & \\
\hline & Int. & Macq. U & 2.85 & 1.62 & 1.91 \\
\hline & Ext. & Macq. U & & & \\
\hline
\end{tabular}

$\mathrm{R}_{\mathrm{c}}$ conversion references: $\mathrm{R}_{\mathrm{c}}(\mathrm{MPI}-1)^{\mathrm{a}}(12), \mathrm{R}_{\mathrm{c}}(\mathrm{MAI})^{\mathrm{b}}(13), \mathrm{R}_{\mathrm{c}}(\mathrm{MDI})^{\mathrm{c}}(13)$. 


\section{Table S10. Selected HyPy yields and ratios of PAH and alkane compounds}

\begin{tabular}{|c|c|c|c|c|c|c|c|}
\hline Sample & $\begin{array}{l}\text { Interior/ } \\
\text { Exterior } \\
\end{array}$ & $\begin{array}{c}\text { Mass } \\
\text { pyrolysed (g) } \\
\end{array}$ & $P(n g / g)^{a}$ & Py $(n g / g)^{a}$ & $\sum \mathbf{M P} / \mathbf{P}^{\mathbf{b}}$ & $\sum \mathrm{MePy} / \mathbf{P y}^{\mathrm{c}}$ & $\begin{array}{c}n \text {-alkanes } \\
(\mathbf{n g} / \mathrm{g})^{\mathrm{d}}\end{array}$ \\
\hline \multirow[t]{2}{*}{$2 / 1 / 001$} & Int. $\# 1$ & 2.002 & 117 & 484 & 1.20 & 0.55 & 332 \\
\hline & Int. \#2 & 2.001 & 105 & 360 & 1.26 & 0.76 & 206 \\
\hline \multirow[t]{2}{*}{ 2/1/003 } & Int. \#1. & 1.288 & 36 & 310 & 0.46 & 0.10 & $<10$ \\
\hline & Int. \#2 & 1.372 & 18 & 275 & 0.42 & 0.08 & 20 \\
\hline \multirow[t]{2}{*}{ 2/1/004 } & Int. \#1 & 2.003 & 428 & 1039 & 1.06 & 0.41 & 166 \\
\hline & Int. \#2 & 2.005 & 357 & 825 & 0.97 & 0.47 & 142 \\
\hline 2/1/007 & Ext. & 1.611 & 68 & 350 & 0.32 & 0.09 & n.d. \\
\hline 2/1/010 & Int. & 2.001 & 3 & 18 & n.d. & 0.06 & $<10$ \\
\hline 2/1/011 & Ext. & 1.519 & $180^{\mathrm{a}}$ & $813^{e}$ & 0.26 & 0.09 & n.d. \\
\hline 3/1/004 & Ext. & 1.519 & 34 & 111 & 0.45 & 0.14 & 33 \\
\hline 3/1/005 & Ext. & 1.995 & 3 & 28 & n.d. & 0.10 & $<10$ \\
\hline Sand bl. & $\# 1$ & 1.874 & $<1$ & $<1$ & n.d. & n.d. & $<10$ \\
\hline Sand bl. & $\# 2$ & 1.677 & $<1$ & $<1$ & n.d. & n.d. & $<10$ \\
\hline
\end{tabular}

${ }^{\mathrm{a}}$ Yields of phenanthrene (P) and pyrene (Py) calculated using molecular ion peak areas (178 Da for P; $202 \mathrm{Da}$ for Py) relative to d14- $p$-terphenyl standard (244 Da) and normalized to unit mass of initial rock pyrolysed. Errors in analyte yields are estimated as $\pm 50 \%$ due to low pyrolysate yield associated with overmature rocks.

${ }^{b}$ Ratio of [(sum of the methylphentherenes)/phenthrene] using appropriate integrated peak areas in 192 $\mathrm{Da}$ ion chromatograms for MP and $178 \mathrm{Da}$ ion chromatograms for P, respectively.

${ }^{\mathrm{c}}$ Ratio of [(sum of the methylpyrenes)/pyrene] using appropriate integrated peak areas in $216 \mathrm{Da}$ for MPy and $202 \mathrm{Da}$ ion chromatograms for Py, respectively.

${ }^{\mathrm{d}}$ Absolute yields of $\mathrm{C}_{12}-\mathrm{C}_{20} n$-alkanes. Yields are likely significantly underestimated for these volatile analytes.

${ }^{\mathrm{e}}$ HyPy run was terminated at $550^{\circ} \mathrm{C}$ rather than $520^{\circ} \mathrm{C}$, which explains the high $\mathrm{P}$ and Py yields

NB: Samples 2/1/001, 2/1/003 and 2/1/004 were pyrolysed as kerogen concentrates following $\mathrm{HCl}$ treatment for carbonate dissolution (see section 2.4.4). The others were ran as extracted rock powders since TOC contents were higher and the carbonate mineral contents were low. 


\section{Chapter 3:}

\section{Organic geochemistry of the early Toarcian oceanic anoxic event in Hawsker Bottoms, Yorkshire, England}

This chapter was published in Earth and Planetary Science Letters (EPSL) by Elsevier in 2014 and is reprinted here with their permission as granted in the original copyright agreement.

French KL, Sepúlveda J, Trabucho-Alexandre J, Gröcke DR, Summons RE (2014) Organic geochemistry of the early Toarcian oceanic anoxic event in Hawsker Bottoms, Yorkshire, England. Earth Planet Sci Lett 390:116-127. 


\title{
Organic geochemistry of the early Toarcian oceanic anoxic event in Hawsker \\ Bottoms, Yorkshire, England
}

\author{
K. L. Frencha ${ }^{*}$, J. Sepúlvedab, J. Trabucho-Alexandrec, D. R. Gröckec, R. E. Summons ${ }^{\mathrm{b}}$
}

a Joint Program in Chemical Oceanography; Massachusetts Institute of Technology and Woods Hole Oceanographic Institution, Cambridge, MA 02139, United States

${ }^{\mathrm{b}}$ Department of Earth, Atmospheric, and Planetary Sciences; Massachusetts Institute of Technology, Cambridge, MA 02139, United States

'Department of Earth Sciences, University of Durham, Durham, DH1 3LE, UK

*Corresponding author:

E-mail address: klfrench@mit.edu (K. L. French)

Tel: +01 617-324-3953

\begin{abstract}
A comprehensive organic geochemical investigation of the Hawsker Bottoms outcrop section in Yorkshire, England has provided new insights about environmental conditions leading into and during the Toarcian oceanic anoxic event (T-OAE; 183 Ma). Rock-Eval and molecular analyses demonstrate that the section is uniformly within the early oil window. Hydrogen index (HI), organic petrography, polycyclic aromatic hydrocarbon $(\mathrm{PAH})$ distributions, and tricyclic terpane ratios mark a shift to a lower relative abundance of terrigenous organic matter supplied to the sampling locality during the onset of the T-OAE and across a lithological transition. Unlike other ancient intervals of anoxia and extinction, biomarker indices of planktonic community structure do not display major changes or anomalous values. Depositional environment and redox indicators support a shift towards more reducing conditions in the sediment porewaters and the development of a seasonally stratified water column during the T-
\end{abstract}


OAE. In addition to carotenoid biomarkers for green sulfur bacteria (GSB), we report the first occurrence of okenane, a marker of purple sulfur bacteria (PSB), in marine samples younger than $\sim 1.64 \mathrm{Ga}$. Based on modern observations, a planktonic source of okenane's precursor, okenone, would require extremely shallow photic zone euxinia (PZE) and a highly restricted depositional environment. However, due to coastal vertical mixing, the lack of planktonic okenone production in modern marine sulfidic environments, and building evidence of okenone production in mat-dwelling Chromatiaceae, we propose a sedimentary source of okenone as an alternative. Lastly, we report the first parallel compound-specific $\delta^{13} \mathrm{C}$ record in marine- and terrestrialderived biomarkers across the T-OAE. The $\delta^{13} \mathrm{C}$ records of short-chain $n$-alkanes, acyclic isoprenoids, and long-chain $n$-alkanes all encode negative carbon isotope excursions (CIEs), and together, they support an injection of isotopically light carbon that impacted both the atmospheric and marine carbon reservoirs. To date, molecular $\delta^{13} \mathrm{C}$ records of the T-OAE display a negative CIE that is smaller in magnitude compared to the bulk organic $\delta^{13} \mathrm{C}$ excursion. Although multiple mechanisms could explain this observation, our molecular, petrographic, and Rock-Eval data suggest that variable mixing of terrigenous and marine organic matter is an important factor affecting the bulk organic $\delta^{13} \mathrm{C}$ records of the T-OAE.

Keywords: Toarcian oceanic anoxic event; lipid biomarkers; okenane; photic zone euxinia, stable carbon isotopes; Hawsker Bottoms 


\subsection{Introduction}

Several transient episodes of enhanced deposition and preservation of organicrich sediments punctuated the Mesozoic Era. A combination of factors may have caused these intervals, known as oceanic anoxic events (OAEs), including greenhouse conditions and enhanced marine productivity (e.g. Schlanger and Jenkyns, 1976; Jenkyns, 1980; 1988; 2010; Trabucho-Alexandre et al., 2010). The first Mesozoic OAE was the Early Jurassic Toarcian OAE (T-OAE; 183 Ma), which was associated with elevated extinction rates, enhanced weathering rates, warm temperatures, ocean acidification, and a negative carbon isotope excursion (CIE) (Hesselbo et al., 2000; Cohen et al., 2004; Bambach, 2006; Hesselbo et al., 2007; Jenkyns, 2010; Kiessling and Simpson, 2011). The duration of the T-OAE is not precisely known but may have lasted on the order of several hundred thousand years (Kemp et al., 2005; Suan et al., 2008; Kemp et al., 2011). The Karoo and Ferrar igneous provinces, which erupted at $183 \pm 1 \mathrm{Ma}$, may have coincided with the Pliensbachian-Toarcian extinction (Pálfy and Smith, 2000; Courtillot and Renne, 2003). However, better radiometric dating of the volcanism and OAE are required to confidently link these two events. Although multiple mechanisms have been proposed to account for the Toarcian negative CIE, including methane hydrate dissociation, upwelling of isotopically light waters, thermogenic release of methane, and biomass burning (Hesselbo et al., 2000; Schouten et al., 2000; McElwain et al., 2005; van de Schootbrugge et al., 2005; Finkelstein et al., 2006), the source of the isotopically light carbon remains unclear.

The analysis of sedimentary organic matter provides the opportunity to evaluate environmental and ecological responses to carbon cycle perturbations, as well as potentially constraining the perturbation itself. Previous organic geochemical work across the T-OAE has indicated changes in planktonic community structure and redox chemistry, particularly the development of photic zone euxinia (PZE) (e.g. Farrimond et 
al., 1989; 1994; Schouten et al., 2000; Pancost et al., 2004; Schwark and Frimmel, 2004;

Bowden et al., 2006; van Breugel et al., 2006). However, since biomarker records can reflect local responses, additional comprehensive organic geochemical studies from multiple locations are required to build a global perspective of ecological and environmental change associated with the T-OAE. Here, we investigate the temporal variation of lipid biomarkers, Rock-Eval data, organic petrography, and compoundspecific carbon isotopes from the Lower Jurassic section at Hawsker Bottoms, Yorkshire, England.

\subsection{Geologic Setting and Site Description of Hawsker Bottoms, Yorkshire, England}

A well-studied section of the Toarcian OAE is located on the Yorkshire coast in northern England. We analyzed sample splits spanning $14 \mathrm{~m}$ of an organic-rich, lower Jurassic outcrop section in the Cleveland Basin at Hawsker Bottoms previously studied by Hesselbo et al. (2000). The lithology is dominated by black shales containing discrete levels of calcite concretions and constitutes the Jet Rock sensu stricto (Hesselbo and Jenkyns, 1995). The sections around Hawsker Bottoms have been used for defining the ammonite biostratigraphy of the Toarcian (Howarth, 1992).

The Early Jurassic paleogeography of the area, although somewhat uncertain, is depicted in published paleogeographic maps (e.g. Bradshaw et al., 1992). The Cleveland Basin of North Yorkshire was part of a system of shallow epicontinental seas and small extensional tectonic basins linked to the Central Graben via the Sole Pit Basin. The region formed part of the broad epicontinental sea that covered much of northwest Europe. Marine sedimentation was initiated during the Late Triassic, and a succession of marine siliciclastic mudstones accumulated during the Early Jurassic.

The Grey Shale Member of the Whitby Mudstone Formation consists of bioturbated, silty mudstones with beds of calcareous siderite concretions. The 
mudstones have thin sharp-based beds, wave ripple, and starved ripple laminations (Wignall et al., 2005; Ghadeer and Macquaker, 2011). Grain size and bioturbation intensity decrease toward the top of the unit, and sediment color darkens. The Jet Rock Member consists of dark, organic matter-rich, fissile mudstones with abundant ammonites and horizons of calcareous nodules. The boundary between these two members of the Whitby Mudstone Formation likely represents an increase in water depth in the basin.

The early Toarcian (D. tenuicostatum Zone) was a period of major basin subsidence throughout England. Organic matter content fluctuates through the Grey Shales, but increasing levels of organic matter are present from the D. semicelatum Subzone to the $C$. exaratum Subzone (H. falciferum Zone). Minor shoaling cycles with striped siltstone laminae suggest that water depths were on the order of tens of meters (Powell, 2010). Similarly, sedimentary structures suggest deposition during storms by the effects of waves (Wignall et al., 2005; Ghadeer and Macquaker, 2011). Thus, bottom water conditions were more energetic than is commonly thought, where the water column was likely shallower than $50 \mathrm{~m}$. Consequently, Hawsker Bottoms likely represents an inner continental shelf environment, which physical oceanographers define as the region where turbulence from the surface and bottom boundary layers effectively homogenizes the whole water column (Lentz and Fewings, 2012). Accordingly, inner shelf environments are typically a few meters to tens of meters deep

The abundance of ammonites in the shales indicates that the water column was at times oxygenated and favorable to nektonic faunas (Powell, 2010). The abundance of thin beds with tops homogenized by bioturbation suggests that long-term, persistent bottom water anoxia did not occur in the basin (Ghadeer and Macquaker, 2011). Besides deposition as bedload by geostrophic flows and density currents, additional sediment was supplied by suspension settling. Textural analyses have shown that much of the sedimentary organic matter was delivered to the seafloor as fecal pellets, flocs, or other 
organo-mineralic aggregates (Ghadeer and Macquaker, 2011). The contribution of a biogenic component to rock composition varies, and the differences have been attributed to a changing balance of primary production relative to dilution and length of transport path during deposition (Macquaker and Taylor, 1996; Wignall et al., 2005; Ghadeer and Macquaker, 2011).

\subsection{Methods}

Powdered rock samples were analyzed by Rock-Eval pyrolysis. The total organic carbon $(\mathrm{TOC} ; \%), \mathrm{T}_{\max }\left({ }^{\circ} \mathrm{C}\right), \mathrm{S}_{1}$, and $\mathrm{S}_{2}$ were determined and used to calculate the hydrogen index (HI) and production index (PI). Kerogen isolates from four samples across the section were mounted onto slides in duplicate and assessed optically under white light and fluorescent light using a Zeiss research microscope and a Zeiss $x 40$ Plank-Neofluar objective. A Zeiss Axioskop, Axio Image D1, and a Zeiss 18 filter set were used to take photomicrographs and fluorescence images.

Powdered samples ( 5 g) were extracted using a Dionex ASE 200 Accelerated Solvent Extractor with a solvent mixture of dichloromethane:methanol 9:1 (v/v). Elemental sulfur was removed from the total lipid extract (TLE). Asphaltenes were separated from the maltene fraction, which was then separated into saturated, aromatic, and polar fractions by silica gel chromatography. The saturated and aromatic fractions were analyzed by gas chromatography-mass spectrometry (GC-MS) and gas chromatography-metastable reaction monitoring-mass spectrometry (GC-MRM-MS).

Carbon isotopic measurements of saturated hydrocarbons were made by gas chromatography/combustion/ isotope ratio mass spectrometry (GC-C-IRMS) using a ThermoFinnigan Delta Plus XP coupled to a ThermoFinnigan Trace GC. The mean value of triplicate analyses are reported here in per mil (\%o) relative to Vienna Pee Dee belemnite (VPDB), and the standard deviation from the mean value was $0.4 \%$ or less. A 
detailed description of methods is included in the supplementary online material (SOM).

\subsection{Results and Discussion}

\subsubsection{Rock-Eval analysis}

Rock-Eval results provided insight into the thermal maturity and type of organic matter preserved in the Hawsker Bottoms sediments. The TOC percentage (Hesselbo et al., 2000) was plotted for comparison with the HI, PI, and $\mathrm{T}_{\max }$ (fig. 1). The narrow range of the PI $(0.11-0.18)$ and $\mathrm{T}_{\max }\left(429-440^{\circ} \mathrm{C}\right)$ parameters indicate that thermal maturity is uniform through the section and at the early stage of oil generation (Peters et al., 2005). Molecular indices of thermal maturity further substantiate this conclusion (see section 3.4.2).

The HI data reveal that the type of organic matter undergoes a transition that appears to coincide with a lithological transition from medium grey shale to dark grey thin-bedded shale within the limitations of our sampling resolution. The low HI values below $-2.5 \mathrm{~m}$ are characteristic of type III kerogen, whereas the higher HI values above $3 \mathrm{~m}$ are characteristic of type II kerogen (Peters et al., 2005). Type III kerogen is dominated by either terrigenous or highly degraded organic matter, and type II kerogen is typically derived from marine organic matter (Peters et al., 2005).

Published HI values from other T-OAE localities also increase across the initiation of the CIE (Prauss et al., 1991; Schouten et al., 2000; Röhl et al., 2001; Sabatino et al., 2009; Suan et al., 2011). Previous workers have attributed the HI variability to different degrees of organic matter degradation under varying redox conditions (Schouten et al., 2000; Röhl et al., 2001; Sabatino et al., 2009). Alternatively, others have argued that the HI variability represents a shift in the composition of the organic matter (Suan et al., 2011). Petrographic and molecular evidence for the presence of plantderived material in these sediments (see section 3.4.3) supports the conclusion that 
lower HI values at the bottom of the section are due to a larger abundance of terrigenous organic matter relative to marine organic matter.

\subsubsection{Molecular indicators of thermal maturity}

The thermal history of the section was further assessed according to molecular thermal maturity parameters (fig. 2). The $\mathrm{C}_{31}$ hopane $22 \mathrm{~S} /(22 \mathrm{~S}+22 \mathrm{R})$ ratio was constant through the section and exhibited a narrow range between 0.58 and 0.59 . A value of $\sim 0.55$ represents the endpoint which is reached around the main phase of oil generation (Peters et al., 2005). The $C_{30}$ hopane $\beta \alpha /(\beta \alpha+\alpha \beta)$ ratio ranged from 0.08 to 0.11 , which is close to values indicative of a mature source rock (Peters et al., 2005). The $\mathrm{C}_{29}$ sterane $\alpha \alpha \alpha 20 S /(20 S+20 \mathrm{R})$ ratio varied from 0.52 to 0.57 , which is comparable to the endpoint value of 0.52-0.55 (Peters et al., 2005). In summary, molecular thermal maturity indicators corroborate the Rock-Eval results, further supporting a uniform thermal maturity within the early window of oil generation.

Some biomarker-based thermal maturity parameters can be influenced by additional factors such as source and diagenetic effects (Moldowan et al., 1986; Dahl et al., 1993; Peters et al., 2005; Bennett and Olsen, 2007; French et al., 2012). Indeed, two of the thermal maturity parameters presented in figure 2 exhibit some variation tracking changes in lithology and source input, despite the multiple lines of evidence supporting constant thermal maturity through the section. The $\mathrm{Ts} /(\mathrm{Ts}+\mathrm{Tm})$ ratio, where $\mathrm{Tm}$ is $\mathrm{C}_{27}$ $17 \alpha$-trisnorhopane and Ts is $C_{27} 18 \alpha$-trisnorhopane, varied from 0.41 to 0.59 , whereas the diasterane/sterane ratio of $\mathrm{C}_{27-29}$ compounds ranged from 1.08 to 1.44 . Both ratios deviate from a relatively constant pattern in the lower $\sim 2$ meters of the section. This pattern is explained by changes in lithology and/or organic matter source input, which

is consistent with lower $\mathrm{HI}$ values and additional evidence supporting variable terrigenous organic input (see section 3.4.3). 


\subsubsection{Biomarker and petrographic evidence of terrigenous organic matter input}

A combination of molecular and petrographic analyses was performed to evaluate the relative contribution of terrigenous organic matter through the sampling interval (fig. 3). Previous work has suggested a terrigenous source for $C_{19}$ and $C_{20}$ tricyclic terpanes, which has led to the use of $C_{19} / C_{23}$ and $C_{20} / C_{23}$ tricyclic terpane ratios to identify input of terrigenous organic matter (Noble et al., 1986; Peters et al., 2005). These two ratios display higher values in the lowest part of the section and decrease after the lithological transition at -2.5 meters, indicating relatively greater terrigenous organic matter input in the lowermost part of the sampling interval.

A wide range of PAHs was detected in the Hawsker Bottoms samples, including phenanthrene, fluoranthene, pyrene, benzo[a]anthracene, triphenylene, chrysene, benzo[b]fluoranthene, benzo[k]fluoranthene, benzo[e]pyrene, indeno[c,d]pyrene, dibenzo[a,h]anthracene, benzo[g,h,i]perylene, coronene, and retene. PAHs are a diverse set of compounds with multiple documented sources including products of pyrolysis, combustion, hydrothermal activity, and igneous intrusion as well as direct inputs from algae, fungi, vascular plants, and extraterrestrial organics (Kawka and Simoneit, 1990; George, 1992; Jiang et al., 2000; Sephton et al., 2005; Grice et al., 2007; Marynowski and Simoneit, 2009). PAHs have been used to reconstruct the history of wildfires, higher plant input, and anthropogenic activity, where peri-condensed, unsubstituted PAHs are markers for combustion of organic matter (Hites et al., 1977; Venkatesan and Dahl, 1989; Killops and Massoud, 1992; Kruge et al., 1994; Jiang et al., 1998; Arinobu et al., 1999; Finkelstein et al., 2005; Peters et al., 2005; Marynowski and Simoneit, 2009). Some sedimentary PAHs, such as phenanthrene, chrysene, and triphenylene, are more affected by diagenesis or additional sources (Jiang et al., 1998; Grice et al., 2007), so they were not included in the total PAH sum plotted in figure $3 \mathrm{~b}$. Although the patterns are not identical, enhanced concentrations of total PAH co-occur with elevated $\mathrm{C}_{19} / \mathrm{C}_{23}$ and $\mathrm{C}_{20} / \mathrm{C}_{23}$ tricyclic terpane ratios in the bottom 2 meters of the sampling interval. Some 
PAHS, such as retene, are thought to derive from higher plants, in particular coniferous resin (Wakeham et al., 1980; Ellis et al., 1996; Jiang et al., 1998; Grice et al., 2005; Peters et al., 2005), although algal and bacterial sources have been reported as well (Wen et al., 2000). Retene was detected in all samples, and its concentration was plotted separately as a marker of higher plant input in figure 3c. Retene was more abundant in the lowest interval of the section, which is consistent with the total PAHs, tricyclic terpane ratios, and HI data.

Microscopic analysis of four kerogen samples adds an additional line of evidence supporting stratigraphic variations in kerogen type and organic matter sources. According to the petrographic results, the kerogen is comprised of as much as $80 \%$ of terrigenous organic matter in the bottom of the section and about $25-40 \%$ in the remainder of the sampling interval (fig. 3d). Since the elemental, molecular, and isotopic composition of organic matter from higher plants is distinct from marine organic matter, $\mathrm{HI}$ can reflect the compositional difference of distinct types of organic matter (e.g. Talbot and Livingstone, 1989). Assuming constant HI values for terrigenous and marine organic matter end-members, the percent terrigenous organic matter was estimated through the section using the linear relationship between the percent terrigenous macerals measured by optical microscopy and the corresponding HI (see SOM for more details). Calculated values of $f_{\text {Terr }}$ OM ranged from 12 to $85 \%$, where the highest values were found in the lowermost part of the section (fig. 3e).

The stratigraphic change in the relative supply of terrigenous organic matter, which is supported by the HI, molecular, and petrographic results, may have been driven by effects related to rising sea level (Hesselbo, 2008; Suan et al., 2011). Considering that increased rates of continental weathering across the T-OAE would have enhanced delivery of terrigenous material (Cohen et al., 2004), the opposite trend recorded in our data might be best explained by progressive remoteness from the coastline on a gently sloping shelf during sea level transgression (e.g. Macquaker et al., 
2010). However, sea level related effects might not be the only factor responsible for the changing signal in relative abundance of terrigenous and marine organic matter. For example, enhanced marine export productivity and/or enhanced marine organic matter preservation could have diluted the input of terrigenous organic matter, thereby changing the relative apparent contribution.

\subsubsection{Biomarker indicators of source and community structure}

Since some compounds or compound classes are associated with a particular biological source, metabolism, or physiology, molecular distributions can be informative about changes in microbial community structure. Unlike other intervals of ocean anoxia associated with mass extinction events (e.g. Xie et al., 2005; Cao et al., 2009), algal- and bacterial-derived biomarkers indicative of community structure did not vary significantly through the CIE (fig. 4). Instead, some biomarker indices that typically reflect community structure were more affected by source input at Hawsker Bottoms.

The regular sterane/17 $\alpha$-hopane ratio is used as an indicator of the relative contributions of eukaryote and bacterial biomass. The regular sterane/hopane ratio was calculated using regular $\mathrm{C}_{27-29}$ steranes and $17 \alpha \mathrm{C}_{29-33}$ hopanes. The regular sterane/17 $\alpha-$ hopane ratio exhibited low values below $-2.5 \mathrm{~m}$, whereas it was more elevated $(>0.5)$ and relatively constant in the top $10 \mathrm{~m}$ of the section. While this offset could be interpreted as a shift from a bacterially dominated environment in the lower part of the section to a eukaryotic environment above $-3 \mathrm{~m}$, it is more likely that a change in the organic matter source input is driving the regular sterane/17 $\alpha$-hopane ratio variability. As well as containing low total steroid abundances, terrigenous organic matter can deliver hopanes derived from soil bacteria, thereby lowering the regular sterane/17 $\alpha$ hopane ratio (Peters et al., 2005; Handley et al., 2010; Sãenz et al., 2011; French et al., 2012). 
Similarly, small deviations are found at the bottom of the section for the $C_{27} / C_{27-30}$, $\mathrm{C}_{28} / \mathrm{C}_{27-30}$, and $\mathrm{C}_{29} / \mathrm{C}_{27-30}$ sterane ratios. These ratios included regular steranes as well as diasteranes and are commonly used as indicators of the relative contribution from red algae biomass, chlorophyll-c algae, and green algae, respectively. However, they can also be affected by the delivery of $\mathrm{C}_{29}$ steranes derived from land plants (Moldowan et al., 1985; Peters et al., 2005). Indeed, $C_{29}$ sterane was the dominant sterane in samples from the bottom of the section, where it represented nearly half of the total $C_{27-30}$ steranes. The $\mathrm{C}_{30} / \mathrm{C}_{27-30}$ sterane ratio, on the other hand, which is an indicator of marine pelagophyte algae, was constant throughout the section and represented only a minor proportion of the total steranes abundance.

The $2 \alpha$-methylhopane index (2-MHI) has been used as an indicator of cyanobacterial input (Summons et al., 1999), although additional sources were later reported (Rashby et al., 2007). The 3 3 -methylhopane index (3-MHI) is considered a marker for aerobic proteobacteria, including methanotrophs and acetic acid bacteria (Zundel and Rohmer, 1985; Talbot et al., 2003; Farrimond et al., 2004; Talbot and Farrimond, 2007). The 2-MHI and 3-MHI were invariant, and the 3-MHI was in the range of average Phanerozoic marine values ( 1-3\%) (Farrimond et al., 2004; Cao et al., 2009). Likewise, the 2-MHI also lacked elevated values. Based on elevated 2-MHI, 3MHI, and nitrogen isotope anomalies, previous workers have reported an increased contribution of diazotrophic cyanobacteria and methanotrophic bacteria during other OAEs (e.g. Kuypers et al., 2004; Cao et al., 2009; Sepúlveda et al., 2009; Luo et al., 2011). The low and invariant contribution of these microbial groups indicates that environmental conditions suitable for their predominance did not prevail at this locality. Thus, enhanced cyanobacterial diazotrophy may not fully explain the previously reported depleted bulk organic $\delta^{15} \mathrm{~N}$ at this location during the T-OAE (Jenkyns et al., 2001). 


\subsubsection{Indicators of redox change and depositional environment}

A suite of biomarkers was used to assess changes in water column stratification and redox potential, including the gammacerane index, $C_{35}$ homohopane index $\left(\mathrm{C}_{35}\right.$ $\mathrm{HHI})$, pristane/phytane $(\mathrm{Pr} / \mathrm{Ph})$ ratio, and the concentration of aromatic carotenoid derivatives (fig. 5). Although some of these parameters can also be influenced by diagenesis, source input, and thermal maturity, they display patterns consistent with a shift towards more intense reducing conditions at least in the sediment porewaters and potentially in the overlying water column.

Although the biological sources of gammacerane are not fully known (Peters et al., 2005), it is a diagenetic product of tetrahymanol, a compound found in bacteriovorous ciliates thriving at the chemocline of stratified water bodies (ten Haven et al., 1989; Sinninghe Damsté et al., 1995). Thus, the occurrence of gammacerane, expressed as the gammacerane index $=$ [gammaceranel (gammacerane $+17 \alpha, 21 \beta C_{30}$ hopane) $]^{*} 100$, has been used to infer changes in water column stratification in ancient environments. Gammacerane was detected in all of the analyzed samples but became more prominent in those deposited during the OAE, starting at the onset of thin-bedded shales.

Elevated abundances of gammacerane during the T-OAE may reflect the development of seasonal water column stratification, possibly due to stronger seasonality and/or deepening of the water column. In contrast, prior to the T-OAE, seasonality may have been weaker, or the water column may have been too shallow to stratify, even during warm months, due to turbulent mixing. The development of seasonal water column stratification at Hawsker Bottoms during the T-OAE would have aided the development of water column oxygen-depletion, particularly during

warm and productive months. However, gammacerane enrichments alone do not necessitate water column anoxia, particularly given the association of its precursor, tetrahymanol, with suboxic waters in the modern (Wakeham et al., 2007; 2012). 
The $\mathrm{C}_{35} \mathrm{HHI}$ and $\mathrm{Pr} / \mathrm{Ph}$ ratio are recorders of depositional redox conditions in sediments. The $\mathrm{C}_{35} \mathrm{HHI}$ records the degree of preservation of the extended side chain of $\mathrm{C}_{35}$ hopanes derived from intact bacteriohopanepolyols (BHPs) (Köster et al., 1997; Peters et al., 2005). Higher $\mathrm{C}_{35} \mathrm{HHI}$ values are characteristic of oxygen-depleted marine depositional environments. Pristane and phytane in ancient marine rock extracts and oils are largely, but not exclusively, derived from the chlorophyll phytyl side chain from photoautotrophs. Redox conditions influence the diagenetic pathway of the phytyl side chain. Reducing conditions promote the conversion of phytol to phytane, and oxic conditions promote the conversion of phytol to pristane (Didyk et al., 1978; Peters et al., 2005). The $\mathrm{C}_{35} \mathrm{HHI}$ nearly doubled in samples deposited during the OAE compared to those deposited prior to the event. Values of the $\mathrm{Pr} / \mathrm{Ph}$ ratio $>3$ recorded at the bottom of the section are suggestive of deposition of terrigenous organic matter under oxic conditions. The $\mathrm{Pr} / \mathrm{Ph}$ ratio values near or below 1 during the T-OAE, together with the elevated $\mathrm{C}_{35} \mathrm{HHI}$, suggest intensification of reducing conditions in the sediment porewaters during deposition.

Biomarkers for anaerobic phototrophic green sulfur bacteria (GSB) have been used to argue for the development of PZE during the T-OAE (Schouten et al., 2000; Pancost et al., 2004; Bowden et al., 2006) and other OAEs (e.g. Cao et al., 2009) based on the physiological requirement of co-occurring reduced sulfur species and light. We detected 2,3,6-aryl isoprenoids, isorenieratane, and chlorobactane in all samples. Unlike previous studies of the T-OAE or any Phanerozoic organic geochemical study of marine samples, trace concentrations of okenane were also identified by GC-MRM-MS in samples above $-3 \mathrm{~m}$, whereas it was below detection limit in samples from the lowest 2 meters of the section. Okenone, a photosynthetic pigment belonging to the PSB family Chromatiaceae, is the only known precursor of okenane (Brocks and Schaeffer, 2008). All compounds were compared with an authentic carotenoid standard and an extract from the Barney Creek Formation (BCF; fig. 6; Brocks et al., 2005). Normalizations of the 
$\mathrm{C}_{40}$ aromatic carotenoid derivatives against the mass of TOC and TLE reveal a similar pattern of elevated concentrations during the anoxic event compared to the pre-event baseline.

In total, all of the organic geochemical redox indicators point towards a shift towards more reducing conditions broadly corresponding with the initiation of the negative CIE. However, sedimentological features, such as starved wave and combined flow ripples, indicate that this area was an energetic, shallow inner shelf environment on the order of tens of meters and probably no deeper than $50 \mathrm{~m}$, where enough oxygen was present in the water column on some timescale to sustain nektonic fauna, including ammonites, and allow for bioturbation at the sediment-water interface through the OAE (Wignall et al., 2005; Powell, 2010; Ghadeer and Macquaker, 2011). We explore different scenarios to reconcile these apparent opposing lines of evidence.

First, the geochemical and sedimentological signals recorded in the rock record are a composite of many processes occurring on different timescales. In the modern ocean, highly productive coastal and continental margin sediments and the overlying water column oscillate between oxic and anoxic conditions over different timescales (e.g. Burdige, 2007). Enhanced productivity and export of organic matter, which are important features of Mesozoic OAEs (e.g. Erba, 2004; Jenkyns, 2010), would have increased the oxygen demand in the water column and sediment porewaters during productive months. During the T-OAE, anoxic conditions may have been restricted to the sediment porewaters during seasons of low productivity, allowing bioturbation to occur when bottom waters were better oxygenated. Conversely, oxygen-deficient waters may have expanded seasonally to the water column during intervals of high productivity and enhanced stratification, as implied by the gammacerane index.

Second, the molecular, paleontological, and sedimentary indicators of redox chemistry apply to different parts of the depositional environment, diagenetic pathways, and have different sensitivities along the redox spectrum. With the exception 
of the GSB and PSB carotenoid markers, the geochemical parameters reported here do not require strict anoxia or euxinia in the water column. The gammacerane index pattern supports the development of seasonal stratification during the OAE, which would have promoted oxygen depletion of the water column. However, the possibility remains that even during intervals of high productivity and stratification, water column oxygen concentrations at this location were depleted but high enough to sustain organisms with physiological oxygen requirements, thereby explaining the fossil and sedimentary evidence. Additionally, the $\mathrm{Pr} / \mathrm{Ph}$ ratio and $\mathrm{C}_{35} \mathrm{HHI}$ pertain primarily to sedimentary redox conditions opposed to water column redox structure. Therefore, the occurrence of intact aromatic carotenoid derivatives merits further discussion to assess water column redox chemistry.

To date, okenane has only been reported in Paleoproterozoic rock extracts and lacustrine Cenozoic extracts (Brocks et al., 2005; Zhang et al., 2011). Given the atmospheric $\mathrm{pO}_{2}$ during the Mesozoic was near present atmospheric levels (Berner, 2006), its detection in marine samples of this age requires careful interpretation. The PSB family Chromatiaceae blooms in a range of anoxic environments with light and reduced sulfur species, including stratified lakes, fjords, coastal lagoons, estuaries, and coastal microbial mats, but not all Chromatiaceae produce okenone (Brocks and Schaeffer, 2008 and references therein). Okenone-producing planktonic Chromatiaceae dwell in water columns where the chemocline is above $25 \mathrm{~m}$ and in $75 \%$ of the reported cases less than $12 \mathrm{~m}$ (Brocks and Schaeffer, 2008). Notably, all of the modern chemocline depth observations for okenone production are based on stratified lake systems. Thus, the lack of okenone in modern marine sulfidic environments presents a "no analog" problem for ancient marine samples containing okenane that were deposited under atmospheric $p \mathrm{O}_{2}$ close to modern levels.

Transient free sulfide has been reported in the water column of intense upwelling zones, including the Arabian Sea, Namibian coast, and the Peruvian coast 
(e.g Dugdale et al., 1977; Brüchert et al., 2003; Naqvi et al., 2006; Schunck et al., 2013). However, these episodes are typically short lived. In contrast, sulfidic waters persist in some restricted marine basins and fjords, including the Black Sea, Cariaco Basin, Saanich Inlet, and the Framvaren and Effingham Fjords. However, these two types of marine environments (non-restricted, transiently sulfidic and restricted, permanently sulfidic) fail to represent suitable modern analogs for Hawsker Bottoms on several counts. Isorenieratene has been measured in the water column and sediments of some restricted marine basins, particularly fjords and the Black Sea (e.g. Sinninghe Damsté and Schouten, 2006), but GSB carotenoids have not been detected in transiently sulfidic upwelling systems. Furthermore, okenone has not been reported in the water column or sediments of any modern marine transiently or permanently sulfidic environment, with the exception of the upper sediments of Kyllaren fjord, a small, highly restricted basin (Smittenberg et al., 2004; Sinninghe Damsté and Schouten, 2006). Like okenone, modern planktonic marine occurrences of chlorobactene are also limited to semi-enclosed water masses that are not representative of fully marine conditions (e.g. Naeher et al. 2012). Interestingly, multiple emerging lines of evidence suggest the occurrence of a "cryptic sulfur cycle" in some OMZs, with a potential role for photosynthetic sulfide oxidation (Canfield et al., 2010; Stewart et al., 2012). However, the presence of GSB and/or PSB and their respective carotenoids have yet to be reported in modern OMZs.

Furthermore, the physical oceanographic processes determining the degree of vertical mixing, hence stratification and redox gradient stability, are markedly different between inner shelf environments and sulfidic, silled basins, which are highly restricted and in many classic modern examples, are an order of magnitude or more deeper than estimated paleodepths of Hawsker Bottoms. Although the water column at Hawsker Bottoms became deeper with the sea level transgression across the T-OAE, the depositional environment remained relatively shallow because of its location on a gently sloping shelf (e.g. Macquaker et al., 2010). Consequently, turbulent mixing at the 
surface and bottom boundary layers would have prevented a stable sulfidic chemocline from developing. On the other hand, considering the limited occurrence of planktonically produced okenone in modern lakes, a planktonic source of okenone at Hawsker Bottoms would imply that, rather than an inner sheld environment, Hawsker Bottoms was a highly restricted coastal basin not reflective of fully marine conditions.

Alternatively, we argue that okenane at Hawsker Bottoms was likely derived from benthic microbial mats based on the lack of modern analogs of okenoneproduction in marine sulfidic environments, the dynamics of inner shelf physical mixing, and building evidence of okenone-producing, mat-dwelling Chromatiaceae (e.g. Caumette et al., 1991; Airs et al., 2001; Caumette et al., 2004; Meyer et al., 2011). Furthermore, planktonic Chlorobiaceae are not the exclusive source of isorenieratene and chlorobactene. Previous work has documented additional non-planktonic GSB sources of isorenieratene and chlorobactene, including microbial mats (e.g.Wahlund et al., 1991; Brocks and Summons, 2003; Beatty et al., 2005; Bühring et al., 2011). Although a mixed planktonic and mat origin of the carotenoids cannot be ruled out, it is more likely that the GSB and PSB carotenoids detected in Hawsker Bottoms samples share a source. Previous studies of sedimentary structures in the Toarcian shales of Yorkshire have attributed wavy laminations to microbial mats (O'Brien, 1990), thereby further supporting a sedimentary origin of GSB and PSB carotenoid derivatives detected in Hawsker Bottoms samples. Similar wavy laminated fabrics have been reported in coeval shales in northern European T-OAE sections (Trabucho-Alexandre et al., 2012). Unfortunately, a sedimentary source of the GSB and PSB carotenoid derivatives offers little information about water column redox chemistry. However, this interpretation does not preclude the development of a suboxic, anoxic, or euxinic water column in this region on some timescale during the T-OAE. Instead, additional inorganic geochemical data is required to better assess the water column redox conditions and degree of basin restriction (Algeo and Tribovillard, 2009). 


\subsubsection{Compound specific stable carbon isotopic data}

A limited number of compound specific $\delta^{13} \mathrm{C}$ records of the T-OAE are currently available in the literature. Here, we report the first long-chain $n$-alkane $\delta^{13} \mathrm{C}$ records of the T-OAE. Compound specific $\delta^{13} \mathrm{C}$ analyses of marine- and terrestrial-derived lipids reveal a shift towards lighter $\delta^{13} \mathrm{C}$ values (fig. 7). Short-chain $n$-alkanes, as well as pristane and phytane are typically used as marine indicators, whereas long-chain $n$ alkanes primarily reflect terrigenous sources. The $n-\mathrm{C}_{17}, n-\mathrm{C}_{18}$, and $n-\mathrm{C}_{19}$ alkanes displayed a negative excursion of $\sim 2-3 \%$, which is consistent with the $\sim 2-4 \%$ o negative excursions documented in the partial $\mathrm{n}-\mathrm{C}_{16-20}$ alkane records from the Toarcian Posidonienschiefer in southwest Germany (Schouten et al., 2000). Pristane and phytane encode a muted excursion ( 1.5-2\%o) compared to short-chain alkane records, and they also have smaller CIE magnitudes compared to the pristane and phytane isotopic records from the Toarcian Paris Basin and the Posidonienschiefer ( 3-4\%o) (Schouten et al., 2000; van Breugel et al., 2006). On the other hand, long-chain $n$-alkanes $\left(n-C_{27}, n-C_{28}\right.$, and $n-C_{29}$ ), which are primarily but not exclusively derived from epicuticular waxes of vascular plants (Eglinton and Hamilton, 1967), display the largest compound specific negative CIE ( 4-5\%). The molecular isotopic records appear to register the initiation of the negative CIE earlier than in the bulk organic record, and within the CIE, the compound-specific $\mathrm{d}^{13} \mathrm{C}$ values remain fairly stable while the bulk curve becomes gradually depleted. However, these features could be due to sampling resolution differences. Higher resolution molecular isotopic records are required to better address the timing and structure of the isotopic excursion recorded in different carbon reservoirs.

The absolute magnitude of the bulk organic CIE $(\sim 5-7 \%)$ is larger than the CIEs recorded in the molecular records from Yorkshire (this study; $1.5-5 \%$ ), the Paris Basin ( 3\%; van Breugel et al., 2006), and the Posidonienschiefer ( $2-4 \%$; Schouten et al., 
2000). Identifying the reason behind the CIE magnitude offsets is critical for placing bounds on the magnitude of isotopically light carbon added into the system. Bulk organic matter is comprised of an array of molecularly and isotopically heterogeneous constituents. In addition to environmental perturbations, organic matter source mixing can contribute to bulk organic $\delta^{13} \mathrm{C}$ excursions (e.g. Pancost et al., 1999). The comparison of short- and long-chain $n$-alkane isotopic compositions demonstrates that, unlike the modern, terrigenous organic matter is isotopically heavier than marine organic matter during the Toarcian (fig. 7), which is consistent with previous Toarcian studies (Vetö et al., 1997; Schouten et al., 2000). Multiple lines of evidence presented in section 3.4.3 highlight a significant transition in the terrigenous organic matter input at Hawsker Bottoms. Indeed, the bottom 2 meters of the study interval are dominated by terrigenous organic matter and are isotopically heavier than the overlying interval that is dominated by marine organic matter. Therefore, an undetermined component of the bulk organic CIE magnitude may be attributed to source mixing effects.

Additional factors could also contribute to the difference in magnitudes between bulk and molecular CIEs. For instance, it is possible that the full CIE was not captured in the molecular isotopic records due to a lower sampling resolution compared to the bulk organic $\delta^{13} \mathrm{C}$ records. Alternatively, water availability can modulate the magnitude of the CIE recorded in vascular leaf waxes, as has been discussed for the Paleocene Eocene Thermal Maximum (PETM; e.g. Schouten et al., 2007; Smith et al., 2007), but unlike the PETM, the ratio of angiosperms and conifers would not account for the $n$ alkane and bulk organic CIE magnitude offset because the rise of angiosperms postdates the Early Jurassic (e.g. Heimhofer et al., 2005). Additionally, thermal maturation could influence the $\delta^{13} \mathrm{C}$ of individual compounds, which become isotopically heavier with increasing thermal maturity (Clayton, 1991; Clayton and Bjorøy, 1994; Tang et al., 2005). 
Nevertheless, while multiple mechanisms may account for the difference between molecular and bulk organic CIE magnitudes, it is significant that a negative CIE is recorded in both marine- and terrestrial-derived lipids, albeit to different degrees. The parallel isotopic change in marine and terrestrial carbon pools recorded at Hawsker Bottoms further supports previous studies suggesting that the T-OAE was a global carbon cycle perturbation where isotopically light carbon entered the atmospheric, terrigenous, and marine carbon reservoirs (Hesselbo et al., 2000; 2007; AlSuwaidi et al., 2010; Caruthers et al., 2011; Gröcke et al., 2011). Multiple sources of isotopically light carbon have been proposed, including methane hydrate dissociation, regional upwelling of isotopically light waters in stratified epicontinental seas, thermogenic release of methane from organic-rich strata in contact with dykes, biomass burning, or a combination of these mechanisms (Hesselbo et al., 2000; Schouten et al., 2000; McElwain et al., 2005; van de Schootbrugge et al., 2005; Finkelstein et al., 2006).

Although our study does not provide evidence in support of a specific forcing mechanism, it allows us to narrow down potential mechanisms. A deep-water source of isotopically light carbon is unlikely because of the CIE observed in land plant biomarkers. The lack of evidence for bacterial methanotrophy in our section suggests that methane hydrate dissociation did not supply appreciable methane to the sampling locality. The organic matter source transition complicates the interpretation of $\mathrm{PAH}$ abundances as tracers of biomass burning, so a different sampling locality without an organic matter source transition should be studied to test the biomass burning mechanism using PAHs. A cascade of mechanisms rather than a single mechanism likely initiated the T-OAE. However, our results indicate that the influence of source mixing on the bulk organic $\delta^{13} \mathrm{C}$ has been previously underestimated and could have potentially affected other bulk isotopic systematics such as nitrogen. Therefore, previous estimates regarding the magnitude of this global perturbation of the carbon cycle should be revisited. 


\subsection{Conclusions}

We use a multiproxy approach based on bulk geochemistry, lipid biomarkers, and compound-specific stable isotopes to elucidate environmental and ecological changes associated with the T-OAE at Hawsker Bottoms in Yorkshire, England. Molecular indicators and Rock-Eval results suggest that thermal maturity is uniformly in the early oil generation window throughout the sampling interval. The HI data, organic petrography, $\mathrm{PAH}$ distribution, and tricyclic terpane ratios indicate a transition in the relative input of terrigenous vs. marine organic matter across a lithological transition. The shift to lower relative abundance of terrigenous organic matter was likely a result of sea level related effects such as coastal proximity, changes in marine organic matter preservation, and/or dilution effects from increased marine productivity.

Organic geochemical redox and depositional environment indicators point towards an overall shift towards more reducing conditions in sediment porewaters and the development of seasonal stratification during the OAE. Previous sedimentological observations require that the water column was not completely anoxic throughout the entire T-OAE, which may seem contradictory at first to the detection of GSB and PSB carotenoids. However, here we present the first occurrence of okenane, a carotenoid marker of PSB, in marine samples younger than the Paleoproterozoic (1.64 Ga). This unexpected finding challenges the interpretation of GSB and PSB carotenoids as markers of PZE in the context of Hawsker Bottoms due to inner shelf vertical mixing, the lack of modern analogs of okenone-production in marine sulfidic environments, and the emerging evidence of okenone-producing mat-dwelling Chromatiaceae. Therefore, in combination with previous reports of microbial wavy lamination in Toarcian shales of Yorkshire and coeval shales in northern Europe, we argue that okenane, and potentially chlorobactane and isorenieratane, was most likely mat-derived at Hawsker Bottoms. 
The compound-specific $\delta^{13} \mathrm{C}$ records of short-chain n-alkanes, acyclic isoprenoids, and long-chain $n$-alkanes support a carbon cycle perturbation that affected both the atmospheric and marine systems, which precludes the recycling of isotopically light $\mathrm{CO}_{2}$ from anoxic waters as the sole mechanism responsible for the T-OAE negative CIE. Notably, compound specific $\delta^{13} \mathrm{C}$ records of the T-OAE, including the new data presented here from Yorkshire and previous molecular data from the Paris Basin and the Posidonienschiefer, encode negative CIEs that are smaller in magnitude compared to bulk organic $\delta^{13} \mathrm{C}$ records. Many mechanisms could contribute to this observation, particularly variable mixing of terrigenous and marine organic matter, which is supported by the multiple lines of evidence for a transition in organic matter source. Identifying the mechanisms behind the CIE magnitude offsets is important for estimating the magnitude of isotopically light carbon injected into the surface carbon reservoirs.

\subsection{Acknowledgements}

We thank Alison Cohen and Emma Grosjean for their contributions to the early stages of this study, Lorraine Eglinton for petrographic analysis, Carolyn Colonero for laboratory assistance, and three anonymous reviewers for valuable comments that improved this work. Funding support for work at MIT was provided by grants from the NASA Astrobiology Institute and the NASA Exobiology Program to RES and an NSF

graduate fellowship to KLF. This project was partly funded by a NSERC Discovery Grant (\# 288321) and NERC Standard Grant (NE/H021868/1) to DRG. JTA is funded by the NERC Standard Grant (NE/H021868/1) to DRG. 


\subsection{References}

Airs, R.L., Atkinson, J.E., Keely, B.J., 2001. Development and application of a high resolution liquid chromatographic method for the analysis of complex pigment distributions. J. Chromatogr. A 917, 167-177.

Algeo, T. J., Tribovillard, N., 2009. Environmental analysis of paleoceanographic systems based on molybdenum-uranium covariation. Chem. Geol. 268, 211-225.

Al-Suwaidi, A.H., Angelozzi, G.N., Baudin, F., Damborenea, S.E., Hesselbo, S.P., Jenkyns, H.C., Mancenido, M.O., Riccardi, A.C., 2010. First record of the Early Toarcian Oceanic Anoxic Event from the Southern Hemisphere, Neuquén Basin, Argentina. J. Geol. Soc. (London) $167,633-636$.

Arinobu, T., Ishiwatari, R., Kaiho, K., Lamolda, M.A., 1999. Spike of pyrosynthetic polycyclic aromatic hydrocarbons associated with an abrupt decrease in $\delta^{13} \mathrm{C}$ of a terrestrial biomarker at the Cretaceous-Tertiary boundary at Caravaca, Spain. Geology 27, 723-726.

Bambach, R.K., 2006. Phanerozoic biodiversity mass extinctions. Annu. Rev. Earth Planet. Sci. $34,127-155$.

Beatty, J., Overmann, J., Lince, M., Manske, A., Lang, A., Blankenship, R., Van Dover, C., Martinson, T., Plumley, F., 2005. An obligately photosynthetic bacterial anaerobe from a deep-sea hydrothermal vent. Proc. Natl. Acad. Sci. U.S.A 102, 9306-9310.

Bennett, B., Olsen, S., 2007. The influence of source depositional conditions on the hydrocarbon and nitrogen compounds in petroleum from central Montana, USA. Org. Geochem. 38, 935956.

Berner, R., 2006. GEOCARBSULF: A combined model for Phanerozoic atmospheric $\mathrm{O}_{2}$ and $\mathrm{CO}_{2}$. Geochim. Cosmochim. Acta 70, 5653-5664.

Bowden, S.A., Farrimond, P., Snape, C.E., Love, G.D., 2006. Compositional differences in biomarker constituents of the hydrocarbon, resin, asphaltene and kerogen fractions: An example from the Jet Rock (Yorkshire, UK). Org. Geochem. 37, 369-383.

Bradshaw, M J, J C W Cope, D W Cripps, D T Donovan, M K Howarth, P F Rawson, I M West, and W A Wimbledon. 1992. "Jurassic." Geol. Soc., London, Memoirs 13, 107-129.

Brocks, J., Love, G., Summons, R., Knoll, A., Logan, G., Bowden, S., 2005. Biomarker evidence for green and purple sulphur bacteria in a stratified Palaeoproterozoic sea. Nature 437, 866870.

Brocks, J., Schaeffer, P., 2008. Okenane, a biomarker for purple sulfur bacteria (Chromatiaceae), and other new carotenoid derivatives from the 1640 Ma Barney Creek Formation. Geochim. Cosmochim. Acta 72, 1396-1414.

Brocks, J.J., Summons, R.E., 2003. Sedimentary hydrocarbons, biomarkers for early life. In: Treatise on Geochemistry, (Elsevier-Pergamon, Oxford). Chpt. 8.03, pp. 63-115.

Brüchert, V., Jørgensen, B.B., Neumann, K., Riechmann, D., Schlösser, M., Schulz, H., 2003. Regulation of bacterial sulfate reduction and hydrogen sulfide fluxes in the central Namibian coastal upwelling zone. Geochim. Cosmochim. Acta 67, 4505-4518.

Burdige, D., 2007. Preservation of organic matter in marine sediments: controls, mechanisms, and an imbalance in sediment organic carbon budgets? Chem. Rev. 107, 467-485.

Bühring, S., Sievert, S., Jonkers, H., Ertefai, T., Elshahed, M., Krumholz, L., Hinrichs, K. -U., 
2011. Insights into chemotaxonomic composition and carbon cycling of phototrophic communities in an artesian sulfur-rich spring (Zodletone, Oklahoma, USA), a possible analog for ancient microbial mat systems. Geobiol. 9, 166-179.

Canfield, D.E., Stewart, F.J., Thamdrup, B., De Brabandere, L., Dalsgaard, T., Delong, E.F., Revsbech, N.P., Ulloa, O., 2010. A Cryptic Sulfur Cycle in Oxygen-Minimum-Zone Waters off the Chilean Coast. Science 330, 1375-1378.

Cao, C., Love, G.D., Hays, L., Wang, W., Shen, S., Summons, R.E., 2009. Biogeochemical evidence for euxinic oceans and ecological disturbance presaging the end-Permian mass extinction event. Earth Planet. Sci. Lett. 281, 188-201.

Caruthers, A.H., Gröcke, D.R., Smith, P.L., 2011. The significance of an Early Jurassic (Toarcian) carbon-isotope excursion in Haida Gwaii (Queen Charlotte Islands), British Columbia, Canada. Earth Planet. Sci. Lett. 307, 19-26.

Caumette, P., Baulaigue, R., Matheron, R., 1991. Thiocapsa halophila sp. nov., a new halophilic phototrophic purple sulfur bacterium. Arch. Microbiol. 155, 170-176.

Caumette, P., Guyoneaud, R., Imhoff, J., Süling, J., Gorlenko, V., 2004. Thiocapsa marina sp. nov., a novel, okenone-containing, purple sulfur bacterium isolated from brackish coastal and marine environments. Int. J. Syst. Evol. Microbiol. 54, 1031-1036.

Clayton, C., 1991. Effect of maturity on carbon isotope ratios of oils and condensates. Org. Geochem. 17, 887-899.

Clayton, C., Bjorøy, M., 1994. Effect of maturity on ${ }^{13} \mathrm{C} /{ }^{12} \mathrm{C}$ ratios of individual compounds in North Sea oils. Org. Geochem. 21, 737-750.

Cohen, A.S., Coe, A.L., Harding, S.M., Schwark, L., 2004. Osmium isotope evidence for the regulation of atmospheric $\mathrm{CO}_{2}$ by continental weathering. Geology 32, 157-160.

Courtillot, V.E., Renne, P.R., 2003. On the ages of flood basalt events. C. R. Geoscience 335, 113140.

Dahl, J., Moldowan, J., M., Sundararaman, P., 1993. Relationship of biomarker distribution to depositional environment: Phosphoria Formation, Montana, USA. Org. Geochem. 20, 10011017.

Didyk, B., Simoneit, B., Brassell, S., Eglinton, G., 1978. Organic geochemical indicators of palaeoenvironmental conditions of sedimentation. Nature 272, 216-222.

Dugdale, R., Goering, J., Barber, R., Smith, R., Packard, T., 1977. Denitrification and hydrogen sulfide in the Peru upwelling region during 1976. Deep-Sea Res. 24, 601-608.

Eglinton, G., Hamilton, R., 1967. Leaf epicutular waxes. Science. 156, 1322-1335.

Ellis, L., Singh, R.K., Alexander, R., Kagi, R.I., 1996. Formation of isohexyl alkylaromatic hydrocarbons from aromatization-rearrangement of terpenoids in the sedimentary environment: A new class of biomarker. Geochim. Cosmochim. Acta 60, 4747-4763.

Erba, E., 2004. Calcareous nannofossils and Mesozoic oceanic anoxic events. Mar. Micropaleontol. 52, 85-106.

Farrimond, P., Eglinton, G., Brassell, S., Jenkyns, H., 1989. Toarcian anoxic event in Europe: an organic geochemical study. Mar. Petrol. Geol. 6, 136-147.

Farrimond, P., Stoddart, D., Jenkyns, H., 1994. An Organic Geochemical Profile of the Toarcian Anoxic Event in Northern Italy. Chem. Geol. 111, 17-33.

Farrimond, P., Talbot, H.M., Watson, D.F., Schulz, L.K., Wilhelms, A., 2004. Methylhopanoids: 
Molecular indicators of ancient bacteria and a petroleum correlation tool. Geochim. Cosmochim. Acta 68, 3873-3882.

Finkelstein, D.B., Pratt, L.M., Brassell, S.C., 2006. Can biomass burning produce a globally significant carbon-isotope excursion in the sedimentary record? Earth Planet. Sci. Lett. 250, 501-510.

Finkelstein, D.B., Pratt, L.M., Curtin, T.M., Brassell, S.C., 2005. Wildfires and seasonal aridity recorded in Late Cretaceous strata from south-eastern Arizona, USA. Sedimentology 52, 587-599.

French, K.L., Tosca, N.J., Cao, C., Summons, R.E., 2012. Diagenetic and detrital origin of moretane anomalies through the Permian-Triassic boundary. Geochim. Cosmochim. Acta 84, 104-125.

George, S.C., 1992. Effect of igneous intrusion on the organic geochemistry of a siltstone and an oil shale horizon in the Midland Valley of Scotland. Org. Geochem. 18, 705-723.

Ghadeer, S.G., Macquaker, J.H.S., 2011. Sediment transport processes in an ancient muddominated succession: a comparison of processes operating in marine offshore settings and anoxic basinal environments. J. Geol. Soc. (London) 168, 1121-1132.

Grice, K., Backhouse, J., Alexander, R., Marshall, N., Logan, G.A., 2005. Correlating terrestrial signatures from biomarker distributions, $\delta^{13} \mathrm{C}$, and palynology in fluvio-deltaic deposits from NW Australia (Triassic-Jurassic). Org. Geochem. 36, 1347-1358.

Grice, K., Nabbefeld, B., Maslen, E., 2007. Source and significance of selected polycyclic aromatic hydrocarbons in sediments (Hovea-3 well, Perth Basin, Western Australia) spanning the Permian-Triassic boundary. Org. Geochem. 38, 1795-1803.

Gröcke, D., Hori, R., Trabucho-Alexandre, J., Kemp, D., Schwark, L., 2011. An open marine record of the Toarcian oceanic event. Solid Earth 2, 245-257.

Handley, L., Talbot, H.M., Cooke, M.P., Anderson, K.E., Wagner, T., 2010. Bacteriohopanepolyols as tracers for continental and marine organic matter supply and phases of enhanced nitrogen cycling on the late Quaternary Congo deep sea fan. Org. Geochem. 41, 910-914.

Heimhofer, U., P. A. Hochuli, S. Burla, J. M. L. Dinis, and H. Weissert., 2005. Timing of Early Cretaceous angiosperm diversification and possible links to major paleoenvironmental change. Geology 33, 141-144.

Hesselbo, S., 2008. Sequence stratigraphy and inferred relative sea-level change from the onshore British Jurassic. P. Geologist Assoc. 119, 19-34.

Hesselbo, S., Jenkyns, H., 1995. A comparison of the Hettangian to Bajocian successions of Dorset and Yorkshire, in: Taylor, P. (Ed.), Field Geology of the British Jurassic. Geological Society of London, pp. 105-150.

Hesselbo, S.P., Gröcke, D.R., Jenkyns, H.C., Bjerrum, C.J., Farrimond, P., Morgans Bell, H.S., Green, O.R., 2000. Massive dissociation of gas hydrate during a Jurassic oceanic anoxic event. Nature 406, 392-395.

Hesselbo, S.P., Jenkyns, H.C., Duarte, L.V., Oliveira, L.C.V., 2007. Carbon-isotope record of the Early Jurassic (Toarcian) Oceanic Anoxic Event from fossil wood and marine carbonate (Lusitanian Basin, Portugal). Earth Planet. Sci. Lett. 253, 455-470.

Hites, R.A., Laflamme, R.E., Farrington, J.W., 1977. Sedimentary Polycyclic Aromatic 
Hydrocarbons: The Historical Record. Science 198, 829-831.

Howarth, M., 1992. The ammonite family Hildoceratidae in the Lower Jurassic of Britain. Palaeontogr. Soc. Monogr. 145, 1-106.

Jenkyns, H., 1988. The early Toarcian (Jurassic) anoxic event: stratigraphic, sedimentary, and geochemical evidence. Am. J. Sci. 288, 101-151.

Jenkyns, H., Gröcke, D., Hesselbo, S., 2001. Nitrogen isotope evidence for water mass denitrification during the early Toarcian (Jurassic) oceanic anoxic event. Paleoceanography 16, 593-603.

Jenkyns, H.C., 1980. Cretaceous anoxic events: from continents to oceans. J. Geol. Soc. (London) $137,171-188$.

Jenkyns, H.C., 2010. Geochemistry of oceanic anoxic events. Geochem. Geophys. Geosyst. 11, Q03004.

Jiang, C., Alexander, R., Kagi, R.I., Murray, A.P., 1998. Polycyclic aromatic hydrocarbons in ancient sediments and their relationships to palaeoclimate. Org. Geochem. 29, 1721-1735.

Jiang, C., Alexander, R., Kagi, R.I., Murray, A.P., 2000. Origin of perylene in ancient sediments and its geological significance. Org. Geochem. 31, 1545-1559.

Kawka, O.E., Simoneit, B.R.T., 1990. Polycyclic aromatic hydrocarbons in hydrothermal petroleums from the Guaymas Basin spreading center. Appl. Geochem. 5, 17-27.

Kemp, D.B., Coe, A.L., Cohen, A.S., Schwark, L., 2005. Astronomical pacing of methane release in the Early Jurassic period. Nature 437, 396-399.

Kemp, D.B., Coe, A.L., Cohen, A.S., Weedon, G.P., 2011. Astronomical forcing and chronology of the early Toarcian (Early Jurassic) oceanic anoxic event in Yorkshire, UK. Paleoceanography 26, PA4210.

Kiessling, W., Simpson, C., 2011. On the potential for ocean acidification to be a general cause of ancient reef crises. Glob. Change Biol. 17, 56-67.

Killops, S.D., Massoud, M.S., 1992. Polycyclic aromatic hydrocarbons of pyrolytic origin in ancient sediments: evidence for Jurassic vegetation fires. Org. Geochem. 18, 1-7.

Köster, J., van Kaam-Peters, H., Koopmans, M., de Leeuw, J., Sinninghe Damsté, J., 1997. Sulphurisation of homohopanoids: Effects on carbon number distribution, speciation, and 22S/22R epimer ratios. Geochim. Cosmochim. Acta 61, 2431-2452.

Kruge, M.A., Stankiewicz, B.A., Crelling, J.C., Montanari, A., Bensley, D.F., 1994. Fossil charcoal in Cretaceous-Tertiary boundary strata: Evidence for catastrophic firestorm and megawave. Geochim. Cosmochim. Acta 58, 1393-1397.

Kuypers, M.M.M., van Breugel, Y., Schouten, S., Erba, E., Sinninghe Damsté, J.S., 2004. N2-fixing cyanobacteria supplied nutrient $\mathrm{N}$ for Cretaceous oceanic anoxic events. Geology 32, 853.

Lentz, S.J., Fewings, M.R., 2012. The Wind- and Wave-Driven Inner-Shelf Circulation. Annu. Rev. Marine. Sci. 4, 317-343.

Luo, G., Wang, Y., Algeo, T.J., Kump, L.R., Bai, X., Yang, H., Yao, L., Xie, S., 2011. Enhanced nitrogen fixation in the immediate aftermath of the latest Permian marine mass extinction. Geology 39, 647-650.

Macquaker, J., Bentley, S.J., Bohacs, K.M., 2010. Wave-enhanced sediment-gravity flows and mud dispersal across continental shelves: Reappraising sediment transport processes operating in ancient mudstone successions. Geology 38, 947-950. 
Macquaker, J., Taylor, K., 1996. A sequence stratigraphic interpretation of a mudstonedominated succession: the Lower Jurassic Cleveland Ironstone Formation. J. Geol. Soc. (London) 153, 759-770.

Marynowski, L., Simoneit, B.R.T., 2009. Widespread Upper Triassic to Lower Jurassic wildfire records from Poland: Evidence from charcoal and pyrolytic polycyclic aromatic hydrocarbons. Palaios 24, 285-298.

McElwain, J.C., Wade-Murphy, J., Hesselbo, S.P., 2005. Changes in carbon dioxide during an oceanic anoxic event linked to intrusion into Gondwana coals. Nature 435, 479-482.

Meyer, K.M., Macalady, J.L., Fulton, J.M., Kump, L.R., Schaperdoth, I., Freeman, K.H., 2011. Carotenoid biomarkers as an imperfect reflection of the anoxygenic phototrophic community in meromictic Fayetteville Green Lake. Geobiol. 9, 321-329.

Moldowan, J.M., Sundararaman, P., Schoell, M., 1986. Sensitivity of biomarker properties to depositional environment and/or source input in the Lower Toarcian of SW-Germany. Org. Geochem. 10, 915-926.

Moldowan, J.M., Seifert, W.K., Gallegos, E.J., 1985. Relationship Between Petroleum Composition and Depositional Environment of Petroleum Source Rocks. Am. Assoc. Petr. Geol. B. 69, 1255-1268.

Naeher, S., Geraga, M., Papatheodorou, G., Ferentinos, G., Kaberi, H., Schubert, C. J., 2012. Environmental variations in a semi-enclosed embayment (Amvrakikos Gulf, Greece)reconstructions based on benthic foraminifera abundance and lipid biomarker pattern. Biogeosciences 9, 5081-5094.

Naqvi, S., Naik, H., Pratihary, A., D'Souza, W., Narvekar, P., Jayakumar, D., Devol, A., Yoshinari, T., Saino, T., 2006. Coastal versus open-ocean denitrification in the Arabian Sea. Biogeosciences 3, 621-633.

Noble, R.A., Alexander, R., Kagi, R.I., Knox, J., 1986. Identification of some diterpenoid hydrocarbons in petroleum. Org. Geochem. 10, 825-829.

O'Brien, N., 1990. Significance of lamination in Toarcian (Lower Jurassic) shales from Yorkshire, Great Britain. Sediment. Geol. 67, 25-34.

Pálfy, J., Smith, P., 2000. Synchrony between Early Jurassic extinction, oceanic anoxic event, and the Karoo-Ferrar flood basalt volcanism. Geology 28, 747-750.

Pancost, R., Crawford, N., Magness, S., Turner, A., Jenkyns, H., Maxwell, J., 2004. Further evidence for the development of photic-zone euxinic conditions during Mesozoic oceanic anoxic events. J. Geol. Soc. (London) 161, 353-364.

Pancost, R., Freeman, K., Patzkowsky, M., 1999. Organic-matter source variation and the expression of a late Middle Ordovician carbon isotope excursion. Geology 27, 1015-1018.

Peters, K.E., Walters, C.C., Moldowan, J.M., 2005. The Biomarker Guide: Biomarkers and Isotopes In Petroleum Systems and Earth History, 2nd ed, The Biomarker Guide: Biomarkers and Isotopes in Petroleum Systems and Earth History. Cambridge University Press.

Powell, J.H., 2010. Jurassic sedimentation in the Cleveland Basin: a review. Proc. Yorks. Geol. Soc. 58, 21-72.

Prauss, M., Ligouis, B., Luterbacher, H., 1991. Organic matter and palynomorphs in the "Posidonienschiefer" (Toarcian, Lower Jurassic) of southern Germany. Geol. Soc., London 
Spec. Publ. 58, 335-351.

Rashby, S., Sessions, A.L., Summons, R.E., Newman, D., 2007. Biosynthesis of 2methylbacteriohopanepolyols by an anoxygenic phototroph. Proc. Natl. Acad. Sci. U.S.A $104,15099$.

Röhl, H., Schmid, A., Oschmann, W., Frimmel, A., Schwark, L., 2001. The Posidonia Shale (Lower Toarcian) of SW-Germany: an oxygen-depleted ecosystem controlled by sea level and palaeoclimate. Palaeogeogr. Palaeoclimatol. Palaeoecol. 165, 27-52.

Sabatino, N., Neri, R., Bellanca, A., Jenkyns, H.C., Baudin, F., Parisi, G., Masetti, D., 2009. Carbon-isotope records of the Early Jurassic (Toarcian) oceanic anoxic event from the Valdorbia (Umbria-Marche Apennines) and Monte Mangart (Julian Alps) sections: palaeoceanographic and stratigraphic implications. Sedimentology 56, 1307-1328.

Sãenz, J.P., Eglinton, T.I., Summons, R.E., 2011. Abundance and structural diversity of bacteriohopanepolyols in suspended particulate matter along a river to ocean transect. Org. Geochem. 42, 774-780.

Schlanger, S.O., Jenkyns, H.C., 1976. Cretaceous oceanic anoxic events: causes and consequences. Geol. Mijnbouw 55, 179-184.

Schouten, S., van Kaam-Peters, H., Rijpstra, W., Schoell, M., Sinninghe Damsté , J., 2000. Effects of an oceanic anoxic event on the stable carbon isotopic composition of Early Toarcian carbon. Am. J. Sci. 300, 1-22.

Schouten, S., Woltering, M., Rijpstra, W.I.C., Sluijs, A., Brinkhuis, H., Sinninghe Damsté, J.S., 2007. The Paleocene-Eocene carbon isotope excursion in higher plant organic matter: Differential fractionation of angiosperms and conifers in the Arctic. Earth Planet. Sci. Lett. $258,581-592$.

Schunck, H., Lavik, G., Desai, D.K., Großkopf, T., Kalvelage, T., Löscher, C.R., Paulmier, A., Contreras, S., Siegel, H., Holtappels, M., Rosenstiel, P., Schilhabel, M.B., Graco, M., Schmitz, R.A., Kuypers, M.M.M., LaRoche, J., 2013. Giant Hydrogen Sulfide Plume in the Oxygen Minimum Zone off Peru Supports Chemolithoautotrophy. PLoS One 8, e68661.

Schwark, L., Frimmel, A., 2004. Chemostratigraphy of the Posidonia Black Shale, SW-Germany. Chem. Geol. 206, 231-248.

Sephton, M.A., Love, G.D., Meredith, W., Snape, C.E., Sun, C.-G., Watson, J.S., 2005. Hydropyrolysis: A new technique for the analysis of macromolecular material in meteorites. Planet. Space Sci. 53, 1280-1286.

Sepúlveda, J., Wendler, J., Leider, A., Kuss, H-J., Summons, R.E., Hinrichs, K-U., 2009. Molecular isotopic evidence of environmental and ecological changes across the Cenomanian-Turonian boundary in the Levant Platform of central Jordan. Org. Geochem. 40, 553-568.

Sinninghe Damsté, J.S., Schouten, S., Biological markers for anoxia in the photic zone of the water column. In: The Handbook of Environmental Chemistry, (Springer-Verlag, Berlin), pp. 127-163.

Sinninghe Damsté, J., Kenig, F., Koopmans, M., Köster, J., Schouten, S., Hayes, J., de Leeuw, J., 1995. Evidence for gammacerane as an indicator of water column stratification. Geochim. Cosmochim. Acta 59, 1895-1900.

Smith, F., Wing, S., Freeman, K., 2007. Magnitude of the carbon isotope excursion at the 
Paleocene-Eocene thermal maximum: The role of plant community change. Earth Planet. Sci. Lett. 262, 50-65.

Smittenberg, R.H., Pancost, R.D., Hopmans, E.C., Paetzel, M., and Sinninghe Damsté, J.S., 2004, A 400-year record of environmental change in an euxinic fjord as revealed by the sedimentary biomarker record: Palaeogeogr. Palaeoclimatol. Palaeoecol. 202, 331-351.

Stewart, F.J., Ulloa, O., DeLong, E.F., 2012. Microbial metatranscriptomics in a permanent marine oxygen minimum zone. Environ. Microbiol. 14, 23-40.

Suan, G., Nikitenko, B.L., Rogov, M.A., Baudin, F., Spangenberg, J.E., Knyazev, V.G., Glinskikh, L.A., Goryacheva, A.A., Adatte, T., Riding, J.B., Föllmi, K.B., Pittet, B., Mattioli, E., Lécuyer, C., 2011. Polar record of Early Jurassic massive carbon injection. Earth Planet. Sci. Lett. 312, 102-113.

Suan, G., Pittet, B., Bour, I., Mattioli, E., Duarte, L., Mailliot, S., 2008. Duration of the Early Toarcian carbon isotope excursion deduced from spectral analysis: Consequence for its possible causes. Earth Planet. Sci. Lett. 267, 666-679.

Summons, R.E., Jahnke, L., Hope, J., Logan, G., 1999. 2-Methylhopanoids as biomarkers for cyanobacterial oxygenic photosynthesis. Nature 23, 85-88.

Talbot, H., Farrimond, P., 2007. Bacterial populations recorded in diverse sedimentary biohopanoid distributions. Org. Geochem. 38, 1212-1225.

Talbot, H.M., Watson, D.F., Pearson, E.J., Farrimond, P., 2003. Diverse biohopanoid compositions of non-marine sediments. Org. Geochem. 34, 1353-1371.

Talbot, M., Livingstone, D., 1989. Hydrogen Index and Carbon Isotopes of Lacustrine OrganicMatter as Lake Level Indicators. Palaeogeogr. Palaeoclimatol. Palaeoecol. 70, 121-137.

Tang, Y., Huang, Y., Ellis, G.S., Wang, Y., Kralert, P.G., Gillaizeau, B., Ma, Q., Hwang, R., 2005. A kinetic model for thermally induced hydrogen and carbon isotope fractionation of individual n-alkanes in crude oil. Geochim. Cosmochim. Acta 69, 4505-4520.

ten Haven, H., Rohmer, M., Rullkötter, J., Bisseret, P., 1989. Tetrahymanol, the most likely precursor of gammacerane, occurs ubiquitously in marine sediments. Geochim. Cosmochim. Acta 53, 3073-3079.

Trabucho-Alexandre, J., Dirkx, R., Veld, H., Klaver, G., de Boer, P., 2012. Toarcian Black Shales in the Dutch Central Graben: Record of Energetic, Variable Depositional Conditions during an Oceanic Anoxic Event. J. Sediment. Res. 82, 104-120.

Trabucho-Alexandre, J., Tuenter, E., Henstra, G., van der Zwan, K., van de Wal, R., Dijkstra, H., de Boer, P., 2010. The mid-Cretaceous North Atlantic nutrient trap: Black shales and OAEs. Paleoceanography 25, PA4201.

van Breugel, Y., Baas, M., Schouten, S., Mattioli, E., Sinninghe Damsté, J.S., 2006. Isorenieratane record in black shales from the Paris Basin, France: Constraints on recycling of respired $\mathrm{CO}_{2}$ as a mechanism for negative carbon isotope shifts during the Toarcian oceanic anoxic event. Paleoceanography 21, PA4220.

van de Schootbrugge, B., McArthur, J.M., Bailey, T.R., Rosenthal, Y., Wright, J.D., Miller, K.G., 2005. Toarcian oceanic anoxic event: An assessment of global causes using belemnite $C$ isotope records. Paleoceanography 20, PA3008.

Venkatesan, M.I., Dahl, J., 1989. Organic geochemical evidence for global fires at the Cretaceous/Tertiary boundary. Nature 338, 57-60. 
Vetö, I., Demeny, A., Hertelendi, E., Hetenyi, M., 1997. Estimation of primary productivity in the Toarcia Tethys- a novel approach based on TOC, reduced sulphur and manganese contents. Palaeogeogr. Palaeoclimatol. Palaeoecol. 132, 355-371.

Wahlund, T., Woese, C., Castenholz, R., Madigan, M., 1991. A thermophilic green sulfur bacterium from New Zealand hot springs, Chlorobium tepidum sp. nov. Arch. Microbiol. 156, 81-90.

Wakeham, S., Schaffner, C., Giger, W., 1980. Polycyclic aromatic hydrocarbons in Recent lake sediments--I. Compounds having anthropogenic origins. Geochim. Cosmochim. Acta 44, 403-413.

Wakeham, S.G., Amann, R., Freeman, K.H., Hopmans, E.C., Jørgensen, B.B., Putnam, I.F., Schouten, S., Sinninghe Damsté, J.S., Talbot, H.M., Woebken, D., 2007. Microbial ecology of the stratified water column of the Black Sea as revealed by a comprehensive biomarker study. Org. Geochem. 38, 2070-2097.

Wakeham, S.G., Turich, C., Schubotz, F., Podlaska, A., Li, X., Varela, R., Astor, Y., Sãenz, J.P, Rush, D., Sinninghe Damsté , J.S., Summons, R.E., Scranton, M.I., Taylor, G. T., Hinrichs, K.U., 2012. Biomarkers, chemistry, and microbiology show chemoautotrophy in a multilayer chemocline in the Cariaco Basin. Deep-Sea Res. I 163, 133-156.

Wen, Z., Ruiyong, W., Radke, M., Qingyu, W., Guoying, S., Zhili, L., 2000. Retene in pyrolysates of algal and bacterial organic matter. Org. Geochem. 31, 757-762.

Wignall, P., Newton, R.J., Little, C.T.S., 2005. The timing of paleoenvironmental change and cause-and-effect relationships during the Early Jurassic mass extinction in Europe. Am. J. Sci. 305, 1014-1032.

Xie, S., Pancost, R., Yin, H., Wang, H., Evershed, R., 2005. Two episodes of microbial change coupled with Permo/Triassic faunal mass extinction. Nature 434, 494-497.

Zhang, C., Zhang, Y., Cai, C., 2011. Aromatic isoprenoids from the 25-65 Ma saline lacustrine formations in the western Qaidam Basin, NW China. Org. Geochem. 42, 851-855.

Zundel, M., Rohmer, M., 1985. Prokaryotic triterpenoids. 1. 3 $\beta$-Methylhopanoids from Acetobacter species and Methylococcus capsulatus. Eur. J. Biochem. 150, 23-27. 


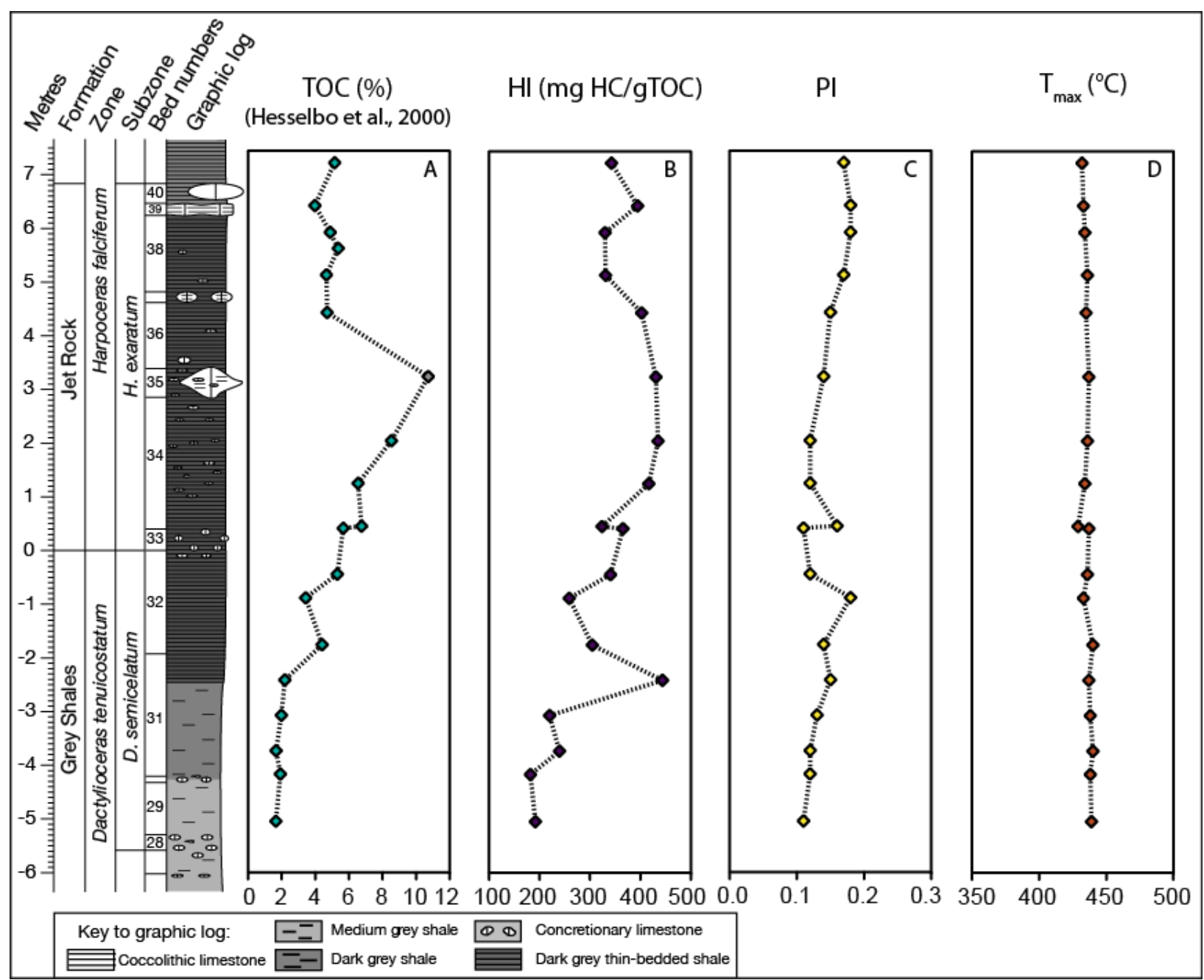

Fig. 1. Rock-Eval Analysis. A) Total organic carbon (TOC; \%) (Hesselbo et al., 2000); B) Hydrogen index (HI; mg HC/g TOC); C) Production index (PI); D) $\mathrm{T}_{\max }\left({ }^{\circ} \mathrm{C}\right)$. 


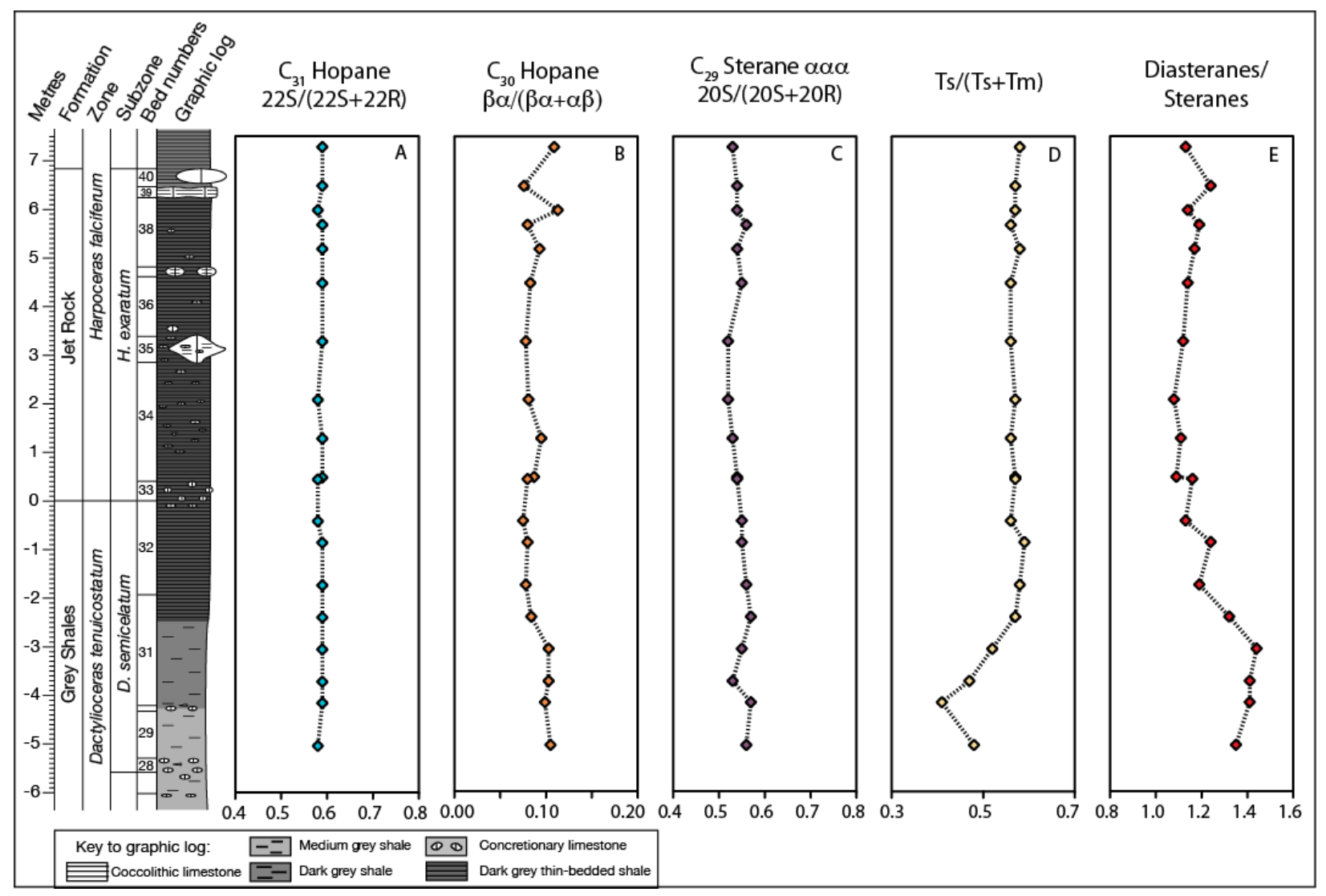

Fig. 2. Molecular Indicators of Thermal Maturity. A) The $\mathrm{C}_{31}$ hopane $22 \mathrm{~S} /(22 \mathrm{~S}+22 \mathrm{R})$ ratio; $\mathrm{B}) \mathrm{C}_{30}$ hopane $\beta \alpha /(\beta \alpha+\alpha \beta)$ ratio; C) $\mathrm{C}_{29}$ sterane $\alpha \alpha \alpha 20 \mathrm{~S} /(20 \mathrm{~S}+20 \mathrm{R})$ ratio; D) $\mathrm{Ts} /(\mathrm{Ts}+\mathrm{Tm})$ ratio; $\mathrm{E})$ diasteranes/steranes ratio. Note the lack of variation in $\mathrm{A}, \mathrm{B}$ and $\mathrm{C}$ across the section compared to the minor variations exhibited by $\mathrm{D}$ and $\mathrm{E}$ due to changes in source input and/or lithology. 


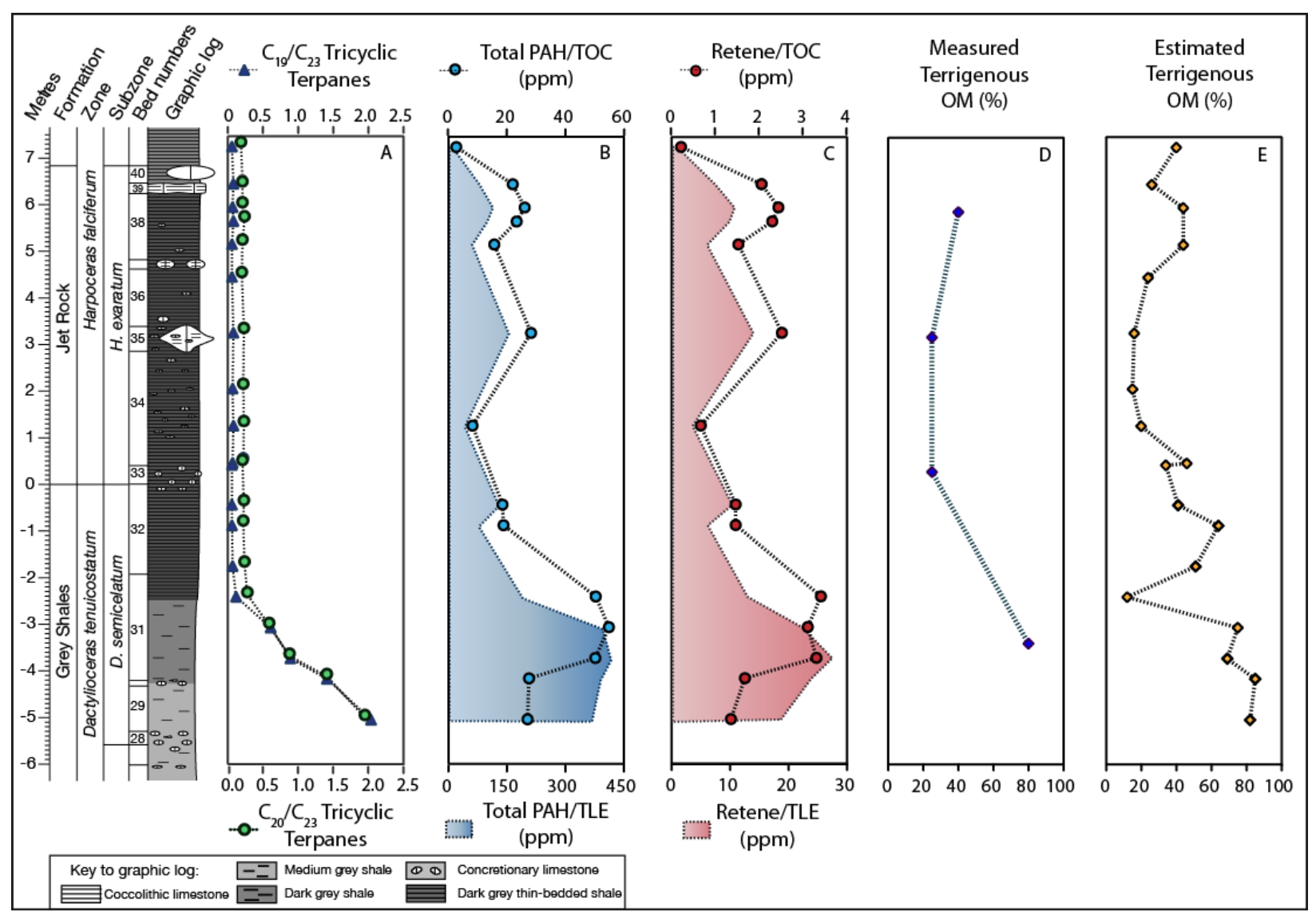

Fig 3. Terrigenous Organic Matter Indicators. A) Ratios of $C_{19} / C_{23}$ and $C_{20} / C_{23}$ tricyclic terpanes; B) total concentration of PAHs normalized by TOC and TLE; C) concentration of retene normalized by TOC and TLE; D) measured contribution of terrigenous organic matter (OM) by petrographic analysis; E) calculated percentage of terrigenous organic matter $\left(f_{\text {Terr OM }}\right)$ based on linear regression ( $f_{\text {Terr OM }}(\%)=-0.279 * \mathrm{HI}+136 ; \mathrm{R}^{2}=0.90$ ) of petrographic measurements of terrigenous organic matter (Fig. 3D) and HI (Fig. 1B). 


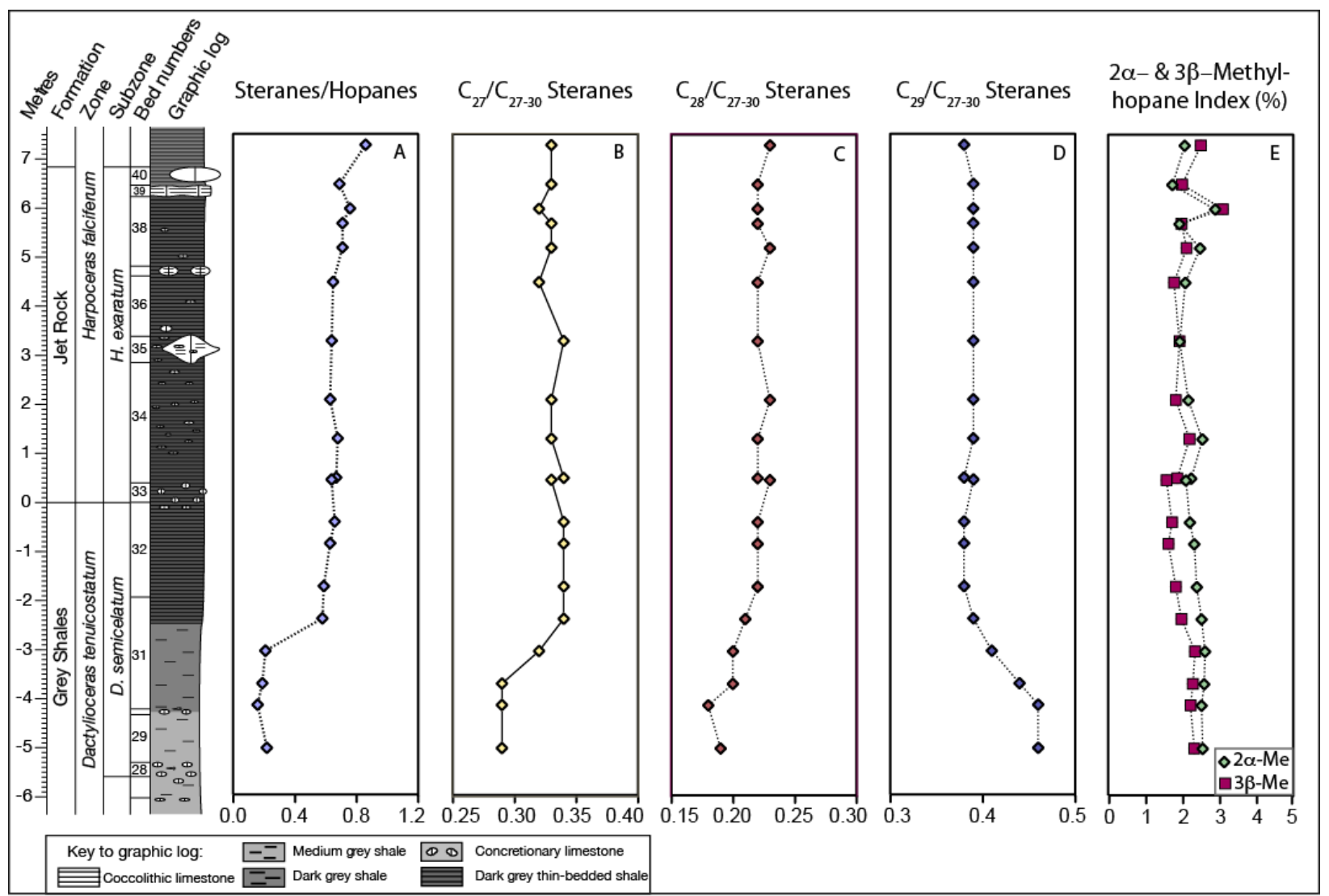

Fig. 4. Source and Community Indicators. A) The ratio of regular steranes/17 $\alpha$ hopanes; B) $\mathrm{C}_{27} / \mathrm{C}_{27-30}$ steranes; C) $\mathrm{C}_{28} / \mathrm{C}_{27-30}$ steranes; D) $\mathrm{C}_{29} / \mathrm{C}_{27-30}$ steranes; E) $2 \alpha-$ methylhopane and $3 \beta$-methylhopane indices. 


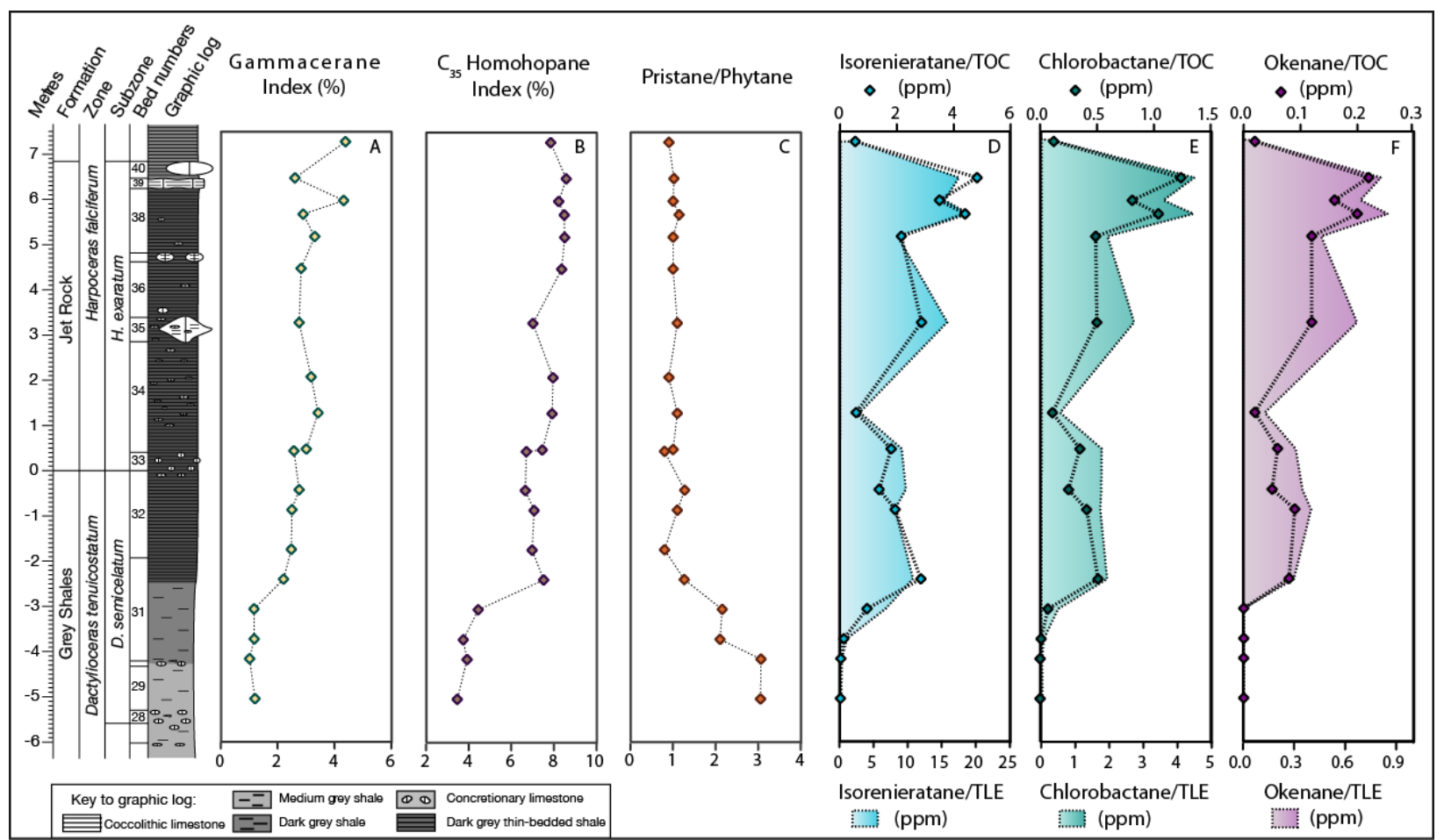

Fig. 5. Redox and Depositional Environmental Indicators. A) Gammacerane index; B) $\mathrm{C}_{35}$ homohopane index $\left.\left(\mathrm{C}_{35} \mathrm{HHI}\right) ; \mathrm{C}\right)$ pristane/phytane $(\mathrm{Pr} / \mathrm{Ph})$ ratio; $\left.\mathrm{D}\right)$ concentration of isorenieratane; E) concentration of chlorobactane; F) concentration of okenane. The concentrations of $\mathrm{C}_{40}$ carotenoids are semi-quantitative and were normalized against TOC and TLE. 


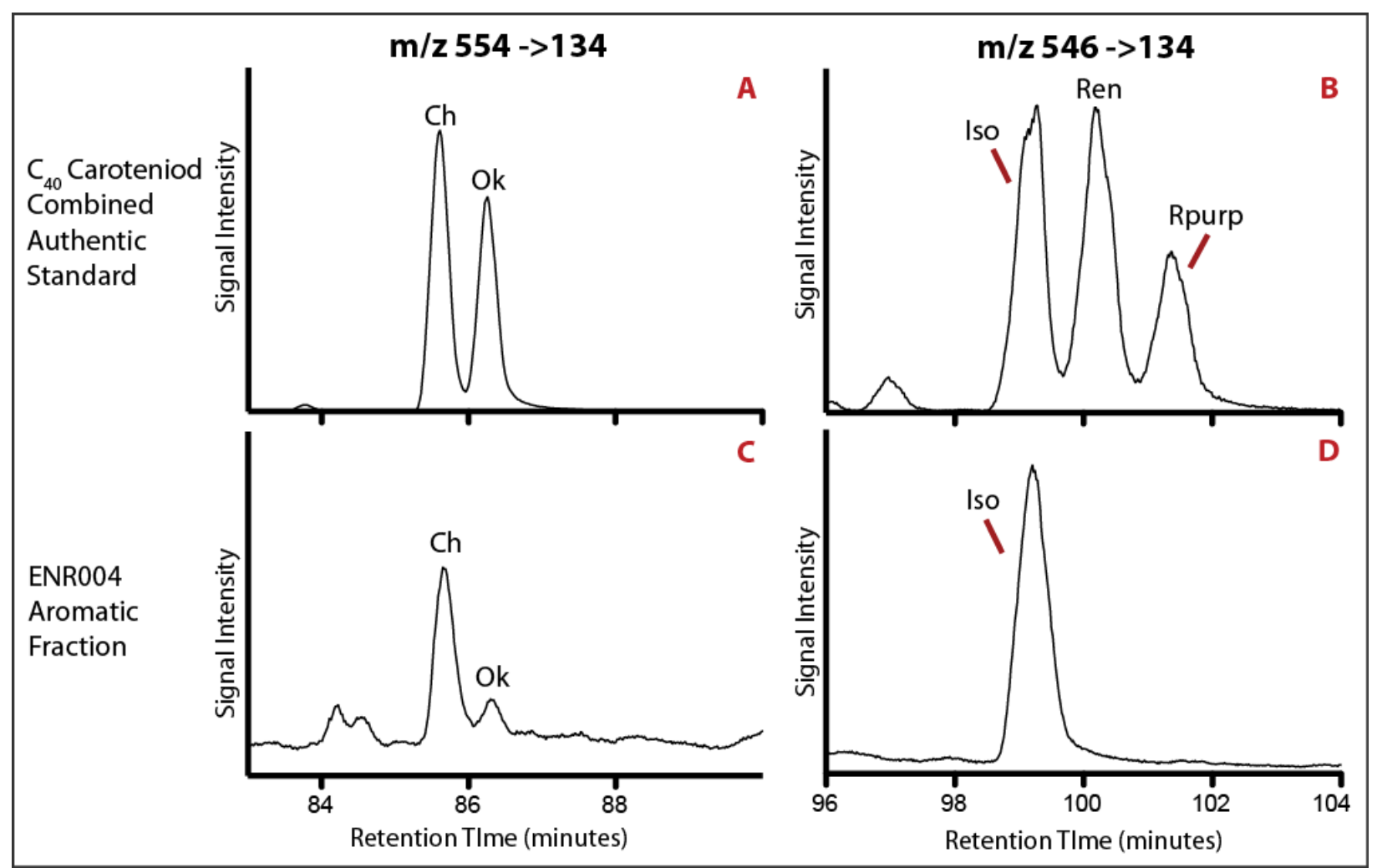

Fig. 6 Gas chromatography - metastable reaction monitoring - mass spectrometry (GC-MRM-MS) chromatogram for the identification of aromatic carotenoid derivatives. MRM chromatograms displaying the $554 \rightarrow 134$ transition characteristic for chlorobactane and okenane in an authentic standard of combined $\mathrm{C}_{40}$ carotenoids (A) and in sample ENR004 at $5.98 \mathrm{~m}$ (C). Plots B and D are MRM chromatograms displaying the $546 \rightarrow 134 \mathrm{MRM}$ transition characteristic of isorenieratane, renieratane, and renierapurpurane in an authentic standard of combined $\mathrm{C}_{40}$ carotenoid (B) and in sample ENR004 (D). Compound abbreviations were used for labels: chlorobactane (Ch); okenane (Ok); isorenieratane (Iso); renieratane (Ren); renierapurpurane (Rpurp). 


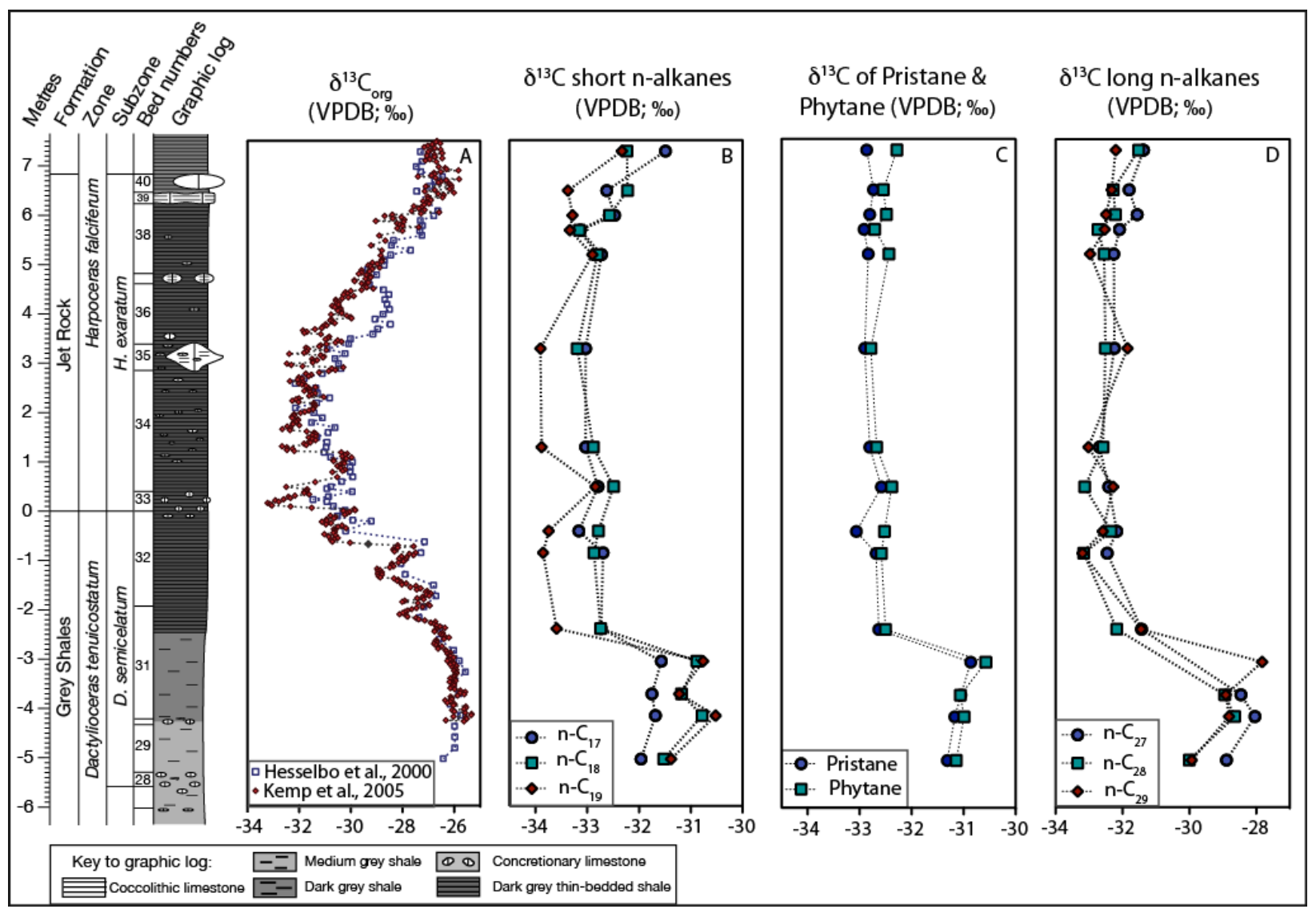

Fig. 7 Carbon Isotopic records. A) Previously reported $\delta^{13}$ Corg from Hawsker Bottoms (Hesselbo et al., 2000; Kemp et al., 2005); B) $\delta^{13} \mathrm{C}$ of $\mathrm{n}-\mathrm{C}_{17-19}$ short-chain $\mathrm{n}$-alkanes; C) $\delta^{13} \mathrm{C}$ of pristane and phytane; D) $\delta^{13} \mathrm{C}$ of $\mathrm{n}-\mathrm{C}_{27-29}$ long-chain $\mathrm{n}$-alkanes. Note the different $\mathrm{x}-$ axis scales. 


\subsection{Supplementary Online Material (SOM)}

\subsubsection{Methods}

Rock-Eval pyrolysis

Powdered sediment samples ( 1 g) were analyzed at the University of Newcastle on a Rock-Eval pyrolysis instrument. The total organic carbon (TOC \%), $\mathrm{T}_{\max }\left({ }^{\circ} \mathrm{C}\right), \mathrm{S}_{1}$, and $S_{2}$ were determined. The $S_{1}$ and $S_{2}$ are expressed in $\mathrm{mg}$ hydrocarbons $(\mathrm{HC})$ per gram dry rock. These parameters were used to calculate the hydrogen index (HI) $\left(\mathrm{HI}=\left[100 * \mathrm{~S}_{2}\right] / \mathrm{TOC}\right.$; expressed in $\mathrm{mg}$ hydrocarbon $\left.(\mathrm{HC}) / \mathrm{g} \mathrm{TOC}\right)$ and the production index (PI) $\left(\mathrm{PI}=\mathrm{S}_{1} /\left[\mathrm{S}_{1}+\mathrm{S}_{2}\right]\right)$. The Rock-Eval TOC values and the bulk $\mathrm{d}^{13} \mathrm{Corg}_{\text {org }}$ have been previously reported (Hesselbo et al., 2000).

\section{Organic petrography}

In order to examine the nature of the organic matter, particularly the fraction of terrigenous organic matter, four samples across the section were prepared for optical analysis of the kerogen. Kerogen was isolated from the sample material remaining after lipid extraction as described in section 3.3.3. The mineral matrix was removed by the sequential addition of $\mathrm{HCl}$ and $\mathrm{HF}$. Samples were centrifuged and rinsed between acid treatments, and the residual matter was rinsed with water and methanol. A subsample of each kerogen sample was mounted onto a slide in duplicate and assessed optically under white light and fluorescent light using a Zeiss research microscope and a Zeiss $\mathrm{x}$ 40 Plan-Neofluar objective. A Zeiss Axioskop, Axio Image D1, and a Zeiss 18 filter set were used to take photomicrographs and fluorescence images.

The fraction of terrigenous organic matter was estimated for the samples that were not analyzed by organic petrography using the linear relationship between the percent terrigenous organic matter measured by optical microscopy and the corresponding HI: 


$$
f_{\text {Terr } \mathrm{OM}}(\%)=-0.279 * \mathrm{HI}+136
$$

where the linear regression had an $\mathrm{R}^{2}$ value of 0.90 and f $_{\text {Terr }}$ ом represents the terrigenous organic matter as a percentage.

Biomarker extraction and analysis

Powdered samples ( 5 g) were extracted using a Dionex ASE 200 Accelerated Solvent Extractor at $1000 \mathrm{psi}$ and $100^{\circ} \mathrm{C}$, with a solvent mixture of dichloromethane:methanol 9:1 (v/v). The total lipid extract (TLE) was reacted with acidactivated copper shots to remove elemental sulfur. Asphaltenes were separated $(3 x)$ from the maltene fraction by precipitation in n-pentane at $4^{\circ} \mathrm{C}$, and after centrifugation at $3000 \mathrm{rpm}$ for 10 minutes. The maltene fraction was then separated into saturated, aromatic, and polar fractions by silica gel chromatography using hexane, 1:1 (v/v) hexane/dichloromethane, and 7:3 (v/v) dichloromethane/methanol.

The saturated fractions were screened by gas chromatography-mass spectrometry (GC-MS) in full scan using an Agilent 6890 GC equipped with a HP6890 autosampler and interfaced to an Agilent 5973 mass spectrometer. Saturated hydrocarbons were also analyzed by gas chromatography-metastable reaction monitoring-mass spectrometry (GC-MRM-MS) on a Micromass Autospec Ultima mass spectrometer coupled with an Agilent 6890N GC. The analysis was carried out with a 60 $\mathrm{m}$ J\&W Scientific DB-1 fused silica capillary column (internal diameter: $0.25 \mathrm{~mm} ; 0.25 \mathrm{~m}$ film thickness) in pulsed splitless mode. The initial GC oven temperature was programmed to $60^{\circ} \mathrm{C}$ (held for 2 minutes), ramped to $150^{\circ} \mathrm{C}$ at $10^{\circ} \mathrm{C} /$ minute, and then to $315^{\circ} \mathrm{C}$ at $3^{\circ} \mathrm{C} /$ minute (held for 24 minutes). The ion source was in EI mode at a temperature of $250^{\circ} \mathrm{C}$, an ionization energy of $70 \mathrm{eV}$, and acceleration voltage of 8000 $\mathrm{kV}$. Tricyclic terpanes and hopanes were identified by MRM using the molecular ion to 
the m/z 191 transitions. Likewise, steranes were identified by MRM using the molecular ion to $\mathrm{m} / \mathrm{z} 217$ transitions.

The aromatic fraction was analyzed by GC-MS in SIM modes. Prior to analysis, $400 \mathrm{ng}$ of an aromatic internal standard, deuterated phenanthrene, was added to each sample. The GC was fitted with a DB-5 stationary phase column, and the GC oven temperature was ramped from $60^{\circ} \mathrm{C}$ to $150^{\circ} \mathrm{C}$ at $20^{\circ} \mathrm{C} /$ minute, and then to $330^{\circ} \mathrm{C}$ at $4^{\circ} \mathrm{C} /$ minute (held for 27 minutes). The aryl isoprenoids and isorenieratane was identified in the m/z 134 ion chromatograms and quantified using the internal standard. Absolute quantification is not possible without taking into account relative response factors but our approach does allow an internally consistent estimation across the sample set.

The aromatic fraction was also analyzed by GC-MS in full scan and MRM modes on a Micromass Autospec Ultima mass spectrometer coupled with an Agilent 6890N GC autospec. The GC was fitted with a DB-5 stationary phase column. Polycyclic aromatic hydrocarbons (PAHs) were identified and quantified in full scan mode by their mass spectra and by comparison with a mix of authentic standards, with the exception of retene, coronene, and triphenylene, which were identified by their mass spectra and relative retention time. Aromatic carotenoid derivatives were also analyzed by GC-MRM-MS using parent-daughter reactions. Isorenieratane, okenane, and chlorobactane were identified in characteristic MRM transitions by comparison of retention times to an extract from the Barney Creek Formation (BCF) and a standard mix of hydrogenated carotenoids containing chlorobactane, okenane, isorenieratane, renieratane, and renierapurpane. Using the MRM data, okenane and chlorobactane were quantified against the GC-MSD quantified isorenieratane. The aromatic carotenoid derivative and PAH concentrations were normalized against mass of TLE and TOC. 
Compound specific carbon isotopic measurements of saturated hydrocarbons were made by gas chromatography/combustion/ isotope ratio mass spectrometry (GCC-IRMS) using a Thermo Finnigan Delta plus XP coupled to a Thermo Finnigan Trace GC. The initial oven temperature was programmed to $60^{\circ} \mathrm{C}$ (held for 3 minutes), ramped to $180^{\circ} \mathrm{C}$ at $10^{\circ} \mathrm{C} / \mathrm{min}$, and then to $320^{\circ} \mathrm{C}$ at $4^{\circ} \mathrm{C} / \mathrm{min}$ (held for 20 minutes). All samples were bracketed by pulses of in house calibrated reference $\mathrm{CO}_{2}$ gas and Oztech calibrated reference $\mathrm{CO}_{2}$ gas. A standard mix of $n$-alkanes (mix A; Arndt Schimmelmann, Indiana University) was analyzed twice a day to monitor the instrument condition. The mean value of triplicate analyses are reported here in permil $(\%)$ relative to Vienna Pee Dee belemnite (VPDB), and the standard deviation from the mean value was better than $0.4 \%$. 
3.8.2 Data Tables

Table S1. Figure 1 Data

\begin{tabular}{lrrrr}
\hline Sample ID & $\begin{array}{c}\text { Depth } \\
(\mathrm{m})\end{array}$ & HI & PI & Tmax \\
\hline ENR005 & 7.28 & 343 & 0.17 & 432 \\
ENR015 & 6.48 & 395 & 0.18 & 433 \\
ENR004 & 5.98 & 330 & 0.18 & 434 \\
ENR014 & 5.68 & & & \\
ENR003 & 5.18 & 331 & 0.17 & 436 \\
ENR002 & 4.48 & 403 & 0.15 & 435 \\
ENR001 & 3.28 & 431 & 0.14 & 437 \\
ENR006 & 2.08 & 435 & 0.12 & 436 \\
ENR007 & 1.28 & 417 & 0.12 & 434 \\
ENR008 & 0.48 & 324 & 0.16 & 429 \\
ENR013 & 0.44 & 366 & 0.11 & 437 \\
ENR012 & -0.42 & 341 & 0.12 & 436 \\
ENR011 & -0.86 & 259 & 0.18 & 433 \\
ENR010 & -1.74 & 305 & 0.14 & 440 \\
ENR017 & -2.4 & 444 & 0.15 & 437 \\
ENR016 & -3.06 & 220 & 0.13 & 438 \\
ENR009 & -3.72 & 240 & 0.12 & 440 \\
ENR018 & -4.16 & 182 & 0.12 & 438 \\
ENR019 & -5.04 & 192 & 0.11 & 439 \\
\hline & & & &
\end{tabular}


Table S2. Figure 2 Data

\begin{tabular}{|c|c|c|c|c|c|c|}
\hline $\begin{array}{c}\text { Sample } \\
\text { ID }\end{array}$ & $\begin{array}{l}\text { Depth } \\
(\mathrm{m})\end{array}$ & $\begin{array}{c}\text { C31 Hop. } \\
22 S /(22 S+22 R) \\
\end{array}$ & $\begin{array}{c}\text { C30 Hop. } \\
\beta \alpha /(\beta \alpha+\alpha \beta)\end{array}$ & $\begin{array}{c}\mathrm{C}_{29} \text { Ster } \alpha \alpha \alpha \\
20 S /(20 \mathrm{~S}+20 \mathrm{R})\end{array}$ & Ts/(Ts+Tm) & Diasteranes/steranes \\
\hline ENR005 & 7.28 & 0.59 & 0.11 & 0.53 & 0.58 & 1.13 \\
\hline ENR015 & 6.48 & 0.59 & 0.08 & 0.54 & 0.57 & 1.24 \\
\hline ENR004 & 5.98 & 0.58 & 0.11 & 0.54 & 0.57 & 1.14 \\
\hline ENR014 & 5.68 & 0.59 & 0.08 & 0.56 & 0.56 & 1.19 \\
\hline ENR003 & 5.18 & 0.59 & 0.09 & 0.54 & 0.58 & 1.17 \\
\hline ENR002 & 4.48 & 0.59 & 0.08 & 0.55 & 0.56 & 1.14 \\
\hline ENR001 & 3.28 & 0.59 & 0.08 & 0.52 & 0.56 & 1.12 \\
\hline ENR006 & 2.08 & 0.58 & 0.08 & 0.52 & 0.57 & 1.08 \\
\hline ENR007 & 1.28 & 0.59 & 0.10 & 0.53 & 0.56 & 1.11 \\
\hline ENR008 & 0.48 & 0.59 & 0.09 & 0.54 & 0.57 & 1.09 \\
\hline ENR013 & 0.44 & 0.58 & 0.08 & 0.54 & 0.57 & 1.16 \\
\hline ENR012 & -0.42 & 0.58 & 0.08 & 0.55 & 0.56 & 1.13 \\
\hline ENR011 & -0.86 & 0.59 & 0.08 & 0.55 & 0.59 & 1.24 \\
\hline ENR010 & -1.74 & 0.59 & 0.08 & 0.56 & 0.58 & 1.19 \\
\hline ENR017 & -2.4 & 0.59 & 0.08 & 0.57 & 0.57 & 1.32 \\
\hline ENR016 & -3.06 & 0.59 & 0.10 & 0.55 & 0.52 & 1.44 \\
\hline ENR009 & -3.72 & 0.59 & 0.10 & 0.53 & 0.47 & 1.41 \\
\hline ENR018 & -4.16 & 0.59 & 0.10 & 0.57 & 0.41 & 1.41 \\
\hline ENR019 & -5.04 & 0.58 & 0.11 & 0.56 & 0.48 & 1.35 \\
\hline
\end{tabular}


Table S3. Figure 3 Data

\begin{tabular}{|c|c|c|c|c|c|c|c|c|c|}
\hline $\begin{array}{l}\text { Sample } \\
\text { ID }\end{array}$ & $\begin{array}{l}\text { Depth } \\
(\mathrm{m})\end{array}$ & $\begin{array}{c}\mathrm{C}_{19} / \mathrm{C}_{23} \\
\text { Tricyclics }\end{array}$ & $\begin{array}{c}\mathrm{C}_{20} / \mathrm{C}_{23} \\
\text { Tricyclics }\end{array}$ & $\begin{array}{c}\text { Total Pyrogenic } \\
\text { PAH/TOC } \\
(\mathrm{ppm})\end{array}$ & $\begin{array}{c}\text { Total Pyrogenic } \\
\text { PAH/TLE } \\
(\mathrm{ppm})\end{array}$ & $\begin{array}{l}\text { Retene/TOC } \\
\quad(\mathrm{ppm})\end{array}$ & $\begin{array}{l}\text { Retene/TLE } \\
\quad(\mathrm{ppm})\end{array}$ & $\begin{array}{c}\text { Measured } \\
\text { Terrigenous } \\
\text { OM }(\%)\end{array}$ & $\begin{array}{c}\text { Estimated } \\
\text { Terrigenous } \\
\text { OM (\%) }\end{array}$ \\
\hline ENR005 & 7.28 & 0.06 & 0.19 & 2.71 & 11.3 & 0.23 & 1 & & 40 \\
\hline ENR015 & 6.48 & 0.08 & 0.21 & 22.0 & 80.2 & 2.07 & 7.5 & & 26 \\
\hline ENR004 & 5.98 & 0.07 & 0.21 & 26.1 & 115 & 2.45 & 10.8 & 40 & 44 \\
\hline ENR014 & 5.68 & 0.08 & 0.24 & 23.4 & 101 & 2.31 & 9.9 & & \\
\hline ENR003 & 5.18 & 0.06 & 0.21 & 15.7 & 62 & 1.54 & 6.1 & & 44 \\
\hline ENR002 & 4.48 & 0.06 & 0.2 & & & & & & 24 \\
\hline ENR001 & 3.28 & 0.08 & 0.23 & 28.3 & 156 & 2.53 & 14 & 25 & 16 \\
\hline ENR006 & 2.08 & 0.07 & 0.22 & & & & & & 15 \\
\hline ENR007 & 1.28 & 0.08 & 0.23 & 8.24 & 43.5 & 0.68 & 3.6 & & 20 \\
\hline ENR008 & 0.48 & 0.07 & 0.22 & & & & & & 46 \\
\hline ENR013 & 0.44 & 0.06 & 0.21 & & & & & 25 & 34 \\
\hline ENR012 & -0.42 & 0.06 & 0.23 & 18.5 & 131 & 1.48 & 10.4 & & 41 \\
\hline ENR011 & -0.86 & 0.06 & 0.22 & 18.8 & 79.3 & 1.48 & 6.2 & & 64 \\
\hline ENR010 & -1.74 & 0.07 & 0.24 & & & & & & 51 \\
\hline ENR017 & -2.4 & 0.12 & 0.28 & 50.4 & 192 & 3.42 & 13 & & 12 \\
\hline ENR016 & -3.06 & 0.61 & 0.59 & 54.9 & 395 & 3.12 & 22.4 & 80 & 75 \\
\hline ENR009 & -3.72 & 0.89 & 0.88 & 50.3 & 417 & 3.32 & 27.5 & & 69 \\
\hline ENR018 & -4.16 & 1.41 & 1.41 & 27.6 & 390 & 1.69 & 23.9 & & 85 \\
\hline ENR019 & -5.04 & 2.04 & 1.95 & 27.1 & 368 & 1.37 & 18.7 & & 82 \\
\hline
\end{tabular}




\section{Table S4. Figure 4 Data}

\begin{tabular}{|c|c|c|c|c|c|c|c|}
\hline $\begin{array}{l}\text { Sample } \\
\text { ID }\end{array}$ & $\begin{array}{l}\text { Depth } \\
(\mathrm{m})\end{array}$ & $\begin{array}{l}\text { Steranes/ } \\
\text { Hopanes }\end{array}$ & $\begin{array}{c}\mathrm{C}_{27} / \mathrm{C}_{27-30} \text { Ster. } \\
\text { Reg+Dia }\end{array}$ & $\begin{array}{c}\mathrm{C}_{28} / \mathrm{C}_{27-30} \text { Ster. } \\
\text { Reg+Dia }\end{array}$ & $\begin{array}{c}\mathrm{C}_{29} / \mathrm{C}_{27-30} \text { Ster. } \\
\text { Reg+Dia }\end{array}$ & $\begin{array}{l}2 \alpha \text { methyl } \\
\text { hop. index }\end{array}$ & $\begin{array}{l}3 \beta \text { methyl } \\
\text { hop. index }\end{array}$ \\
\hline ENR005 & 7.28 & 0.86 & 0.33 & 0.23 & 0.38 & 2.05 & 2.51 \\
\hline ENR015 & 6.48 & 0.69 & 0.33 & 0.22 & 0.39 & 1.72 & 2 \\
\hline ENR004 & 5.98 & 0.76 & 0.32 & 0.22 & 0.39 & 2.88 & 3.1 \\
\hline ENR014 & 5.68 & 0.71 & 0.33 & 0.22 & 0.39 & 1.91 & 1.98 \\
\hline ENR003 & 5.18 & 0.71 & 0.33 & 0.23 & 0.39 & 2.47 & 2.12 \\
\hline ENR002 & 4.48 & 0.65 & 0.32 & 0.22 & 0.39 & 2.07 & 1.78 \\
\hline ENR001 & 3.28 & 0.64 & 0.34 & 0.22 & 0.39 & 1.92 & 1.93 \\
\hline ENR006 & 2.08 & 0.63 & 0.33 & 0.23 & 0.39 & 2.15 & 1.83 \\
\hline ENR007 & 1.28 & 0.68 & 0.33 & 0.22 & 0.39 & 2.53 & 2.2 \\
\hline ENR008 & 0.48 & 0.67 & 0.34 & 0.22 & 0.38 & 2.23 & 1.87 \\
\hline ENR013 & 0.44 & 0.64 & 0.33 & 0.23 & 0.39 & 2.09 & 1.58 \\
\hline ENR012 & -0.42 & 0.66 & 0.34 & 0.22 & 0.38 & 2.19 & 1.73 \\
\hline ENR011 & -0.86 & 0.63 & 0.34 & 0.22 & 0.38 & 2.31 & 1.63 \\
\hline ENR010 & -1.74 & 0.59 & 0.34 & 0.22 & 0.38 & 2.38 & 1.83 \\
\hline ENR017 & -2.4 & 0.58 & 0.34 & 0.21 & 0.39 & 2.51 & 1.98 \\
\hline ENR016 & -3.06 & 0.21 & 0.32 & 0.2 & 0.41 & 2.6 & 2.35 \\
\hline ENR009 & -3.72 & 0.19 & 0.29 & 0.2 & 0.44 & 2.58 & 2.29 \\
\hline ENR018 & -4.16 & 0.16 & 0.29 & 0.18 & 0.46 & 2.51 & 2.23 \\
\hline ENR019 & -5.04 & 0.22 & 0.29 & 0.19 & 0.46 & 2.54 & 2.33 \\
\hline
\end{tabular}


Table S5. Figure 5 Data

\begin{tabular}{|c|c|c|c|c|c|c|c|c|c|c|}
\hline $\begin{array}{l}\text { Sample } \\
\text { ID }\end{array}$ & $\begin{array}{l}\text { Depth } \\
(\mathrm{m})\end{array}$ & $\begin{array}{c}\text { Gammacerane } \\
\text { Index }\end{array}$ & $\begin{array}{l}\text { C }_{35} \text { hop/ } \\
\text { C }_{31}-C_{35}\end{array}$ & $\mathrm{Pr} / \mathrm{Ph}$ & $\begin{array}{l}\text { Iso ug/g } \\
\text { TOC }\end{array}$ & $\begin{array}{c}\text { Iso } \mathrm{ng} / \mathrm{mg} \\
\text { TLE }\end{array}$ & $\begin{array}{l}\text { Chl ug/ } \\
\text { g TOC }\end{array}$ & $\begin{array}{l}\text { Chl ng/ } \\
\text { mg TLE }\end{array}$ & $\begin{array}{l}\text { Ok ug/g } \\
\text { TOC }\end{array}$ & $\begin{array}{l}\text { Ok ng/ } \\
\text { mg TLE }\end{array}$ \\
\hline ENR005 & 7.28 & 4.38 & 7.84 & 0.9 & 0.54 & 2.24 & 0.12 & 0.48 & 0.02 & 0.09 \\
\hline ENR015 & 6.48 & 2.6 & 8.57 & 1.02 & 4.82 & 17.5 & 1.24 & 4.5 & 0.22 & 0.81 \\
\hline ENR004 & 5.98 & 4.32 & 8.22 & 1 & 3.5 & 15.4 & 0.81 & 3.59 & 0.16 & 0.69 \\
\hline ENR014 & 5.68 & 2.89 & 8.48 & 1.14 & 4.4 & 19.0 & 1.04 & 4.47 & 0.2 & 0.85 \\
\hline ENR003 & 5.18 & 3.3 & 8.5 & 1 & 2.16 & 8.54 & 0.49 & 1.95 & 0.12 & 0.46 \\
\hline ENR002 & 4.48 & 2.82 & 8.36 & 1 & & & & & & \\
\hline ENR001 & 3.28 & 2.75 & 7.01 & 1.1 & 2.87 & 15.8 & 0.50 & 2.73 & 0.12 & 0.67 \\
\hline ENR006 & 2.08 & 3.17 & 7.95 & 0.9 & & & & & & \\
\hline ENR007 & 1.28 & 3.42 & 7.91 & 1.1 & 0.57 & 2.99 & 0.11 & 0.56 & 0.02 & 0.13 \\
\hline ENR008 & 0.48 & 3 & 7.45 & 1 & 1.8 & 9.15 & 0.35 & 1.78 & 0.06 & 0.31 \\
\hline ENR013 & 0.44 & 2.57 & 6.7 & 0.8 & & & & & & \\
\hline ENR012 & -0.42 & 2.75 & 6.65 & 1.27 & 1.38 & 9.75 & 0.25 & 1.77 & 0.05 & 0.35 \\
\hline ENR011 & -0.86 & 2.5 & 7.06 & 1.1 & 1.94 & 8.16 & 0.41 & 1.74 & 0.09 & 0.4 \\
\hline ENR010 & -1.74 & 2.48 & 6.97 & 0.8 & & & & & & \\
\hline ENR017 & -2.4 & 2.2 & 7.51 & 1.26 & 2.85 & 10.8 & 0.51 & 1.94 & 0.08 & 0.3 \\
\hline ENR016 & -3.06 & 1.16 & 4.44 & 2.15 & 0.95 & 6.85 & 0.07 & 0.5 & 0 & 0 \\
\hline ENR009 & -3.72 & 1.17 & 3.74 & 2.1 & 0.14 & 1.14 & 0.01 & 0.08 & 0 & 0 \\
\hline ENR018 & -4.16 & 1.01 & 3.92 & 3.06 & 0.03 & 0.36 & $<0.01$ & 0.03 & 0 & 0 \\
\hline ENR019 & -5.04 & 1.2 & 3.46 & 3.05 & 0.02 & 0.31 & $<0.01$ & 0.02 & 0 & 0 \\
\hline
\end{tabular}


Table S6. Figure 7 Data

\begin{tabular}{|c|c|c|c|c|c|c|c|c|c|}
\hline $\begin{array}{c}\text { Sample } \\
\text { ID }\end{array}$ & Depth (m) & $\begin{array}{l}\text { Avg. } \delta^{13} \mathrm{C} \\
\text { of } n-\mathrm{C}_{17}\end{array}$ & $\begin{array}{c}\text { Avg. } \delta^{13} \mathrm{C} \text { of } \\
n-\mathrm{C}_{18}\end{array}$ & $\begin{array}{c}\text { Avg. } \delta^{13} \mathrm{C} \\
n-\mathrm{C}_{19}\end{array}$ & $\begin{array}{c}\text { Avg. } \delta^{13} \mathrm{C} \\
\operatorname{Pr}\end{array}$ & $\begin{array}{c}\text { Avg. } \\
\delta^{13} \mathrm{C} P h\end{array}$ & $\begin{array}{c}\text { Avg. } \delta^{13} C \\
n-C_{27}\end{array}$ & $\begin{array}{c}\text { Avg. } \delta^{13} \mathrm{C} \\
n-\mathrm{C}_{28}\end{array}$ & $\begin{array}{c}\text { Avg. } \delta^{13} \mathrm{C} \\
n-\mathrm{C}_{29}\end{array}$ \\
\hline ENR005 & 7.28 & -31.5 & -32.2 & -32.3 & -32.9 & -32.3 & -31.4 & -31.5 & -32.2 \\
\hline ENR015 & 6.48 & -32.6 & -32.2 & -33.4 & -32.7 & -32.5 & -31.8 & -32.3 & -32.3 \\
\hline ENR004 & 5.98 & -32.5 & -32.6 & -33.3 & -32.8 & -32.5 & -31.6 & -32.2 & -32.5 \\
\hline ENR014 & 5.68 & -33.1 & -33.2 & -33.3 & -32.9 & -32.7 & -32.1 & -32.7 & -32.5 \\
\hline ENR003 & 5.18 & -32.7 & -32.8 & -32.9 & -32.8 & -32.4 & -32.3 & -32.6 & -33.0 \\
\hline ENR001 & 3.28 & -33.0 & -33.2 & -33.9 & -32.9 & -32.8 & -32.2 & -32.5 & -31.9 \\
\hline ENR007 & 1.28 & -33.0 & -32.9 & -33.9 & -32.8 & -32.7 & -32.7 & -32.6 & -33.0 \\
\hline ENR008 & 0.48 & -32.8 & -32.5 & -32.8 & -32.6 & -32.4 & -32.4 & -33.1 & -32.3 \\
\hline ENR012 & -0.42 & -33.2 & -32.8 & -33.7 & -33.1 & -32.5 & -32.2 & -32.3 & -32.6 \\
\hline ENR011 & -0.86 & -32.7 & -32.9 & -33.9 & -32.7 & -32.6 & -32.5 & -33.2 & -33.2 \\
\hline ENR017 & -2.4 & -32.7 & -32.7 & -33.6 & -32.6 & -32.5 & -31.4 & -32.2 & -31.4 \\
\hline ENR016 & -3.06 & -31.6 & -30.9 & -30.8 & -30.9 & -30.6 & & & -27.8 \\
\hline ENR009 & -3.72 & -31.8 & -31.2 & -31.2 & -31.1 & -31.1 & -28.5 & -28.9 & -28.9 \\
\hline ENR018 & -4.16 & -31.7 & -30.8 & -30.5 & -31.2 & -31.0 & -28.0 & -28.7 & -28.8 \\
\hline ENR019 & -5.04 & -32.0 & -31.5 & -31.4 & -31.3 & -31.1 & -28.9 & -30.0 & -29.9 \\
\hline
\end{tabular}




\section{Chapter 4:}

\section{Assessing the distribution of sedimentary $\mathrm{C}_{40}$ carotenoids through time}

This chapter is in review at Geobiology. 


\title{
Assessing the distribution of sedimentary $\mathrm{C}_{40}$ carotenoids through
}

time

\section{Katherine L. French ${ }^{1 *}$, D. Rocher ${ }^{2}$, J. E. Zumberge ${ }^{2}$, and Roger E. Summons ${ }^{3}$}

1. Joint Program in Chemical Oceanography; Massachusetts Institute of Technology and Woods Hole Oceanographic Institution, Cambridge, MA 02139, USA

2. GeoMark Research, Ltd., Houston, TX 77095, USA

3. Department of Earth, Atmospheric, and Planetary Sciences; Massachusetts Institute of Technology, Cambridge, MA 02139, USA

Corresponding author: klfrench@mit.edu

\begin{abstract}
A comprehensive biomarker record of green and purple sulfur bacteria (GSB and PSB, respectively) in marine systems is required to test whether the prevalence of anoxygenic photosynthesis is coherent with models of ocean redox evolution through geologic time. For this purpose, marine rock extracts and oils from the Proterozoic to the Paleogene were analyzed for saturated and aromatic $\mathrm{C}_{40}$ carotenoid carbon skeletons using new analytical methods. Gas chromatography coupled with tandem mass spectrometry revealed new temporal distributions for okenane, chlorobactane, and paleorenieratane. According to conventional paleoredox interpretations, the revised stratigraphic distribution of the GSB and PSB biomarkers implies that marine photic zone euxinia (PZE) occurred more frequently in the surface ocean $(<24 \mathrm{~m})$ in the geologic past than was previously thought.
\end{abstract}

We reexamine what the evidence is supporting a planktonic source of okenone and chlorobactene in marine water columns with atmospheric oxygen levels near modern values. Accordingly, we review modern environmental distributions of GSB 
and PSB and their corresponding carotenoids in order to evaluate all possible explanations and sources for GSB and PSB carotenoids, and we propose alternative interpretations of GSB and PSB carotenoids in the geologic record. To date, their biological precursors, okenone and chlorobactene, have been identified in restricted coastal embayments, fjords, and lake systems, but they have yet to be detected in unrestricted, fully marine environments despite the existence of transiently sulfidic marine systems in the modern ocean. We propose that in some cases the GSB and PSB biomarkers may not reflect an autochthonous PZE water column signature, but they could instead reflect alternative environmental features, including basin restriction, mat communities, or transported organic matter. Additional studies of cultured taxa, modern environments, and transport pathways are required to assess the range of phototrophic sulfur bacterial carotenoid sources.

\subsection{INTRODUCTION}

The diagenetic products of pigments from GSB and PSB are common paleoredox proxies used to identify past intervals of PZE (e.g. Summons and Powell, 1986; Brocks et al., 2005). This is due to the dual physiological requirements of GSB and PSB for light and reduced sulfur species (Overmann, 2008; Bryant et al., 2012). Nearly three decades of organic geochemical studies reveal isorenieratane as the most widely distributed sedimentary aromatic $\mathrm{C}_{40}$ carotenoid in marine sediments, marine rock extracts, and marine petroleum samples through geologic time (e.g. Sinninghe Damsté and Schouten, 2006), so it is not surprising that it is the primary aromatic $C_{40}$ carotenoid that is targeted as a photic zone euxinia (PZE) marker in paleoenvironmental reconstructions. However, other sedimentary $\mathrm{C}_{40}$ carotenoids can offer valuable information (Fig. 1), such as tighter constraints on depth of hydrogen sulfide penetration into the surface ocean and the source of the $\mathrm{C}_{40}$ carotenoid assemblage as a whole (e.g. planktonic vs. mat). In comparison to isorenieratane, the aromatic $\mathrm{C}_{40}$ carotenoids chlorobactane, 
okenane and paleorenieratane are rarely reported or are even thought to be limited to specific time intervals. Part of the interpretative power gained by the detection of okenane or paleorenieratane stems from their infrequent and narrow stratigraphic occurrence, according to the current literature.

Models of Earth's redox history predict that anoxygenic photosynthesis represented a greater proportion of marine primary production during the Proterozoic compared to the Phanerozoic (Johnston et al., 2009). While the stratigraphic distribution of GSB and PSB carotenoid biomarkers provide an independent test of this prediction, the GSB and PSB biomarker records in marine systems have never been compiled and evaluated. Moreover, examining the stratigraphic distributions of GSB and PSB biomarkers could highlight gaps and weaknesses in the conventional interpretation of this biomarker class. Here, we offer a new perspective on the stratigraphic distribution of aromatic $\mathrm{C}_{40}$ carotenoids in ancient marine systems afforded by analyses of more than 50 sedimentary rock extracts and oils from the Proterozoic to the Paleogene by two tandem mass spectrometry techniques: gas chromatography-mass spectrometry metastable reaction monitoring (GC-MRM-MS) and triple quadrupole (GC-QQQ-MS).

\subsection{GSB and PSB carotenoid sources}

Chlorobactane is a monoaromatic $\mathrm{C}_{40}$ carotenoid derived from the pigment chlorobactene, which is characteristic of the green species of the GSB. In contrast, the precursor of the diaromatic $\mathrm{C}_{40}$ carotenoid isorenieratane, isorenieratene, derives from the brown GSB species, although not exclusively (Maresca et al., 2008; Bryant et al., 2012). While brown GSB can live in very low light conditions as deep as $100 \mathrm{~m}$ in stratified basins (Repeta et al., 1989; Overmann et al., 1992) and in deep sea hydrothermal vent communities (Beatty et al., 2005; Sylvan et al., 2012), the green GSB require significantly higher light intensities and dwell higher in the water column (Vila and Abella, 1994). Consequently, the detection of chlorobactane in geologic samples has 
been used to argue for euxinia at depths as shallow as $15 \mathrm{~m}$ in ancient marine water columns (e.g. Kuypers et al., 2002). GSB living planktonically and in microbial mats both produce chlorobactene (e.g. Burke and Burton, 1988; Wahlund et al., 1991).

Okenone has been documented in at least eight genera of the Chromatiaceae family of PSB and is the only known precursor of the reduced monoaromatic $\mathrm{C}_{40}$ carotenoid okenane (Brocks and Schaeffer, 2008; Hamilton et al., 2014). According to observations of modern systems, planktonic Chromatiaceae that produce okenone grow in water columns where the oxic/anoxic boundary is $<24 \mathrm{~m}$ and in most cases $<12 \mathrm{~m}$ (Brocks and Schaeffer, 2008; Meyer et al., 2011; Hamilton et al., 2014). Okenane is a proposed marker of planktonic Chromatiaceae, but the potential contribution of okenane from mat-dwelling Chromatiaceae in the geologic record is currently debated (e.g. Caumette et al., 1991; Caumette et al., 2004; Brocks and Schaeffer, 2008; Meyer et al., 2011; Hamilton et al., 2014). Okenane was first reported in rock extracts from the Barney Creek Formation (BCF; 1.64 Ga) (Brocks et al., 2005). Until recently, okenane had not been reported in marine sedimentary rock extracts or oils younger than the Paleoproterozoic (Brocks et al., 2005; French et al., 2014, Saito et al., 2014).

The biological precursor and source of paleorenieratane, which is a 2,3,6-/3,4,5trimethyl substituted $\mathrm{C}_{40}$ diaromatic carotenoid, are unknown, but its carbon isotopic composition and frequent co-occurrence with and structural similarity to isorenieratane suggests Chlorobi, or an extinct but related taxon, as a potential source (Hartgers et al., 1994). Paleorenieratane is regarded as an "extinct" biomarker that only occurs in the Paleozoic (Requejo et al., 1992; Hartgers et al., 1994; Koopmans et al., 1996a), so it has been used as an age-specific diagnostic marker (e.g. Rashid and Grosjean, 2006; Melendez et al., 2013). 


\subsection{MATERIALS AND METHODS}

Sedimentary aromatic carotenoids are typically analyzed by GC-MS in selected ion monitoring (SIM) mode, but GC-MRM-MS has been previously used to detect aryl isoprenoids (Summons and Powell, 1992; Blumenberg et al., 2012). Instead of focusing on aryl isoprenoids, which are less source-specific than the intact $\mathrm{C}_{40}$ carotenoids (Koopmans et al., 1996b), we specifically target the sedimentary $\mathrm{C}_{40}$ carotenoids. Here, we report the application of GC-MRM-MS and GC-QQQ-MS to analyze saturated and aromatic sedimentary $\mathrm{C}_{40}$ carotenoids simultaneously. For the purpose of this study, more than 50 rock extracts and oils were selected to cover a broad range of time. Table 1 describes the samples that were analyzed for this study by GC-MRM-MS and GC-QQQMS. Aromatic, saturated, branched/cyclic, and hydrocarbon (i.e. only the polar fraction was removed) fractions from rock extracts and oils were analyzed by GC-MS in MRM mode using a Waters Micromass Autospec Ultima. Air peaks were added to the mass calibration in order to detect the low $\mathrm{M}^{*}\left(\mathrm{M}^{*}=\mathrm{M} 2^{2} / \mathrm{M} 1\right)$ values for the diagnostic 540-560 $\mathrm{Da} \rightarrow 120-135$ Da first field free region fragmentations of intact $\mathrm{C}_{40}$ carotenoids in double sector mass spectrometers. The GC-QQQ-MS was operated in multiple reaction monitoring mode using an Agilent 7000A Triple Quad equipped with an Agilent 7890A gas chromatograph. A more detailed description of analytical conditions is given in the supplementary online material (SOM).

Chlorobactane and okenane were identified as the first and second peaks, respectively, of a doublet in chromatograms for the $554 \rightarrow 134$ MRM/QQQ transition, whereas paleorenieratane eluted before isorenieratane in the $546 \rightarrow 134 \mathrm{MRM} / \mathrm{QQQ}$ transition (Fig. 2). With the exception of paleorenieratane and $\gamma$-carotane, compound identification for the MRM analysis was confirmed by co-elution experiments with synthetic standards and a rock extract from the BCF (Brocks et al., 2005). The identification of paleorenieratane for MRM analysis was confirmed by co-elution experiments with an Canning Basin Devonian oil sample, Blina-1, which contains 
paleorenieratane and by comparison of a full scan mass spectrum with published mass spectra (Requejo et al., 1992). All carotenoid identification by GC-QQQ-MS was achieved by comparison of mass spectra and relative retention times to published full scan data.

Analysis of a subset of rock extracts and oils by both MRM and QQQ demonstrates that both methods were able to detect the same sedimentary carotenoids in identical samples (Fig. 2). Furthermore, simultaneous detection of saturated and aromatic $\mathrm{C}_{40}$ carotenoids by MRM and QQQ methods in a single analysis of a total hydrocarbon fraction, where only the polar and asphaltene fractions were removed, could prove more efficient in future studies (Fig. 3). The MRM and QQQ methods can detect saturated $\mathrm{C}_{40}$ carotanes ( $\beta$-carotane and $\gamma$-carotane) in either a total hydrocarbon fraction or in a saturated hydrocarbon fraction. Likewise, both methods could detect diaromatic $\mathrm{C}_{40}$ carotenoids (isorenieratane, renieratane, and renierapurpurane) and monoaromatic $\mathrm{C}_{40}$ carotenoids (chlorobactane and okenane) in either a total hydrocarbon fraction or in an aromatic hydrocarbon fraction. There is no significant difference in saturated carotane or aromatic carotenoid detection due to whether the hydrocarbon fraction is analyzed in a single analysis or whether the saturated or branched/cyclic fractions are analyzed separately from the aromatic fraction (Fig. 3). Only MRM chromatograms are used to illustrate this point in figure 3, although the same point applies to the QQQ analytical method. Lastly, GC-MRM-MS analysis of a dilution series of standards indicates that saturated $\mathrm{C}_{40}$ carotanes, diaromatic $\mathrm{C}_{40}$ carotenoids, and monoaromatic $\mathrm{C}_{40}$ carotenoids can be detected to concentrations as low as $\sim 10 \mathrm{pg}$.

\subsection{RESULTS AND DISCUSSION}

The complete $\mathrm{C}_{40}$ carotenoid results for our sample set are summarized in Table 1. Sedimentary $\mathrm{C}_{40}$ carotenoids were detected in numerous rock extracts and oils of a 
broad age range. As is commonly the case, isorenieratane or paleorenieratane were the dominant aromatic $\mathrm{C}_{40}$ compounds in most samples that we analyzed. However, a different distribution is observed in some rare cases, such as the Paleoproterozoic BCF samples (Brocks and Schaeffer, 2008), Ediacaran-Cambrian boundary South Oman Salt Basin samples (this study), and Early Triassic samples (Saito et al., 2014). In these samples, isorenieratane is either below detection or in relatively low abundance compared to co-occurring chlorobactane and okenane.

While aromatic $\mathrm{C}_{40}$ carotenoids were detected in many samples, they were not detected in every sample (Table 1; Fig. 4). For instance, they were not detected in a marine rock extract of the Early Cambrian Ouldburra Formation. Likewise, the aromatic $\mathrm{C}_{40}$ carotenoids were not detected in the rock extracts of the saline lacustrine Parakeelya Alkali Member of the Observatory Hill Formation, but $\beta$-carotane was present in these samples. Every sample was analyzed for aromatic $\mathrm{C}_{40}$ carotenoids, but not all of these samples were analyzed for $\mathrm{C}_{40}$ carotanes. However, $\beta$-carotane was present in many samples that were analyzed for both carotenoid classes (Table 1).

\section{Stratigraphic Distribution of Aromatic $\mathrm{C}_{40}$ Carotenoids}

Sinninghe Damsté and Schouten (2006) and Meyer and Kump (2008) documented the occurrence of aromatic carotenoid derivatives in ancient depositional systems. These lists were updated and modified to map published occurrences of isorenieratane, chlorobactane, paleorenieratane, and okenane only in marine environments through geologic time (Fig. 4A). The published occurrences used to construct figure $4 \mathrm{~A}$ are listed and described with their references in Table S1. Combining the GC-MRM-MS and GC-QQQ-MS results with the published occurrences demonstrates that these compounds are surprisingly ubiquitous in ancient marine samples (Fig. 4B). While this study focuses on the more diagnostic $\mathrm{C}_{40}$ carotenoids (Koopmans et al., 1996b), aryl isoprenoid occurrences in ancient depositional systems 
are documented elsewhere (Sinninghe Damsté and Schouten, 2006; Meyer and Kump, 2008).

The Paleoproterozoic still marks the first recorded occurrence of isorenieratane, chlorobactane, and okenane, but the record of chlorobactane was extended through the Paleozoic and into the Neoproterozoic. Okenane was detected in multiple Phanerozoic marine oils and rock extracts. Paleorenieratane was detected in samples spanning the Neoproterozoic into the Early Triassic, suggesting it is not a robust age-specific Paleozoic marker. According to Hartgers et al. (1994), unpublished results suggest that paleorenieratane may even extend into the Mesozoic, although we cannot confirm those observations based on the present dataset.

The broader stratigraphic distribution of aromatic $\mathrm{C}_{40}$ carotenoids is surprising. However, it is important to note that figure 4 reflects a combination of factors and biases, and that the record is incomplete. Paleoenvironmental and depositional factors are, in part, responsible for the occurrence of $\mathrm{C}_{40}$ carotenoids in geologic samples, but diagenetic conditions and thermal maturity are additional controls on carotenoid preservation, detectability, and representation in geologic time. The early oil window $\left(\mathrm{T}_{\max }<\sim 445^{\circ} \mathrm{C}\right.$ ) appears to represent the upper limit for intact $\mathrm{C}_{40}$ carotenoid preservation (e.g. Requejo et al., 1992; Lee and Brocks, 2011). Furthermore, sulfur can significantly affect the diagenetic pathways and preservation potential of carotenoids where a significant fraction of carotenoids can be bound to macromolecular organic matter (Sinninghe Damsté and Schouten, 2006). The Mesozoic has greater aromatic $\mathrm{C}_{40}$ carotenoid density compared to other time intervals according to figure $4 \mathrm{~B}$, but this feature could be a product of preservation biases and an emphasis on OAE paleoredox research. In contrast, an absence of GSB and PSB carotenoids during specific intervals, such as the Carboniferous, may reflect a gap in stratigraphic coverage achieved by our sample set, rather than a real environmental absence of GSB and PSB during that interval. In some cases where aromatic $\mathrm{C}_{40}$ carotenoids were not detected, only a limited 
number of samples were analyzed from that rock formation. However, stratigraphic variability can be significant, meaning that future studies may detect aromatic $\mathrm{C}_{40}$ carotenoids in some of the rock formations where we did not find them.

Future studies should use the described tandem mass spectrometry methods to screen extractable and macromolecularly-bound hydrocarbons with a thermal maturity below the peak oil window, regardless of age, for the full suite of $\mathrm{C}_{40}$ carotenoids as opposed to just isorenieratane and the less specific aryl isoprenoids (Koopmans et al., 1996b). Measuring both aromatic and saturated $C_{40}$ carotenoids will help to distinguish samples that do not contain GSB and PSB carotenoids because the environmental and depositional conditions were not suitable for their production from samples where diagenetic conditions or thermal maturity prevented carotenoid preservation (i.e. neither saturated nor aromatic $\mathrm{C}_{40}$ carotenoids are found). In doing so, some of the biases reflected in figure 4 will be minimized, and it will be possible to test features such as the prevalence of okenane in the Mesozoic or the timing of extinction of paleorenieratane. While additional measurements will add greater density to Fig. 4A, the unexpected stratigraphic distribution of the aromatic $\mathrm{C}_{40}$ carotenoids has important implications for their utility as autochthonous water column paleoredox proxies.

\section{Implications of Temporal Distribution}

Models of ocean paleoredox chemistry predict that anoxygenic photoautotrophy represented a greater fraction of marine production in the Proterozoic relative to the Phanerozoic (Johnston et al., 2009). Shallow marine euxinia $(<24 \mathrm{~m})$ in the Paleoproterozoic, implied by okenane and chlorobactane, is coherent with inferred models of ocean redox chemistry and low atmospheric oxygen levels (Lyons et al., 2009; Planavsky et al., 2014). However, the aromatic $\mathrm{C}_{40}$ carotenoid distribution does match the first order prediction that the occurrence of PSB markers decreases in the oxygenated Phanerozoic oceans (Johnston et al., 2009). According to our analyses, 
Chromatiaceae continued to proliferate in some marine environments after early Paleozoic marine oxygenation.

There are several factors that can explain why the GSB and PSB biomarkers do not match this first order prediction. For example, there are fewer rocks that have low enough thermal maturity for carotenoid preservation in the Precambrian compared to the Phanerozoic. This type of preservation bias gives rise to weaker stratigraphic coverage in the Precambrian and likely contributes to the more infrequent carotenoid occurrence in the Proterozoic relative to the Phanerozoic. Alternatively, a ferrigenous deep ocean during the Proterozoic could also impact the GSB and PSB carotenoid distribution (Sperling et al., 2013; Lyons et al., 2014), such as their apparent absence in the Velkerri Formation of the Roper Group.

The broader stratigraphic distribution of okenane and chlorobactane could mean that marine PZE developed in the surface ocean $(<24 \mathrm{~m})$ more often in the Phanerozoic than was previously thought. Chlorobactane, which was previously reported during the Cenomanian-Turonian oceanic anoxic event (OAE-2) in offshore marine environments where total water depths were estimated to be on the order of hundreds of meters, was interpreted as reflecting an autochthonous PZE water column signature (Kuypers et al., 2002; van Bentum et al., 2009). In this case, seasonal shoaling of the mixed layer depth (MLD) might account for the occurrence of chlorobactane since the MLD shoals to $20 \mathrm{~m}$ or less in nearly half of the modern open ocean, particularly in upwelling and tropical regions (Holte et al. 2010). However, marine sediments deposited under several hundreds to thousands of meters of water become exceedingly rare prior to the Cretaceous. As a result, many studies rely on coastal and ocean margin depositional environments where turbulent mixing tends to hinder the development of a stable and shallow sulfidic chemocline, which is required to sustain PSB and GSB water column production of okenone and chlorobactene. However, because proxy interpretations must have their foundations in modern environmental observations, experimental 
measurements, and/or modeling results, we can evaluate those lines of evidence to inform the interpretation of GSB and PSB carotenoids as markers for water column PZE.

Models of the Permian-Triassic ocean can generate sulfidic water columns when upwelling rates and phosphate supply are sufficient, which could support isorenieratane observations in ancient sedimentary rocks (Meyer et al., 2008). Even in these models, however, the wind mixed surface ocean remains oxygenated (Meyer et al., 2008), which complicates a water column interpretation of chlorobactane and okenane. A shallow wind mixed surface layer, even seasonally, could potentially accommodate planktonic GSB and PSB production of chlorobactene and okenone. However, models do not address photic zone variability, leaving an important physiological requirement unconstrained. This could be significant given that satellite images illustrate that elemental sulfur formation clouds the water column during sulfide eruptions on the Namibian margin (Weeks et al., 2002). For these reasons, modeling evidence alone does not definitively justify a planktonic source for okenone and chlorobactene GSB and PSB production in unrestricted marine environments. Further support is required from observational evidence in modern systems.

To date, the only known marine planktonic production of okenone and chlorobactene occurs in small, semi-enclosed fjords and gulfs (Smittenberg et al., 2004; Naeher et al., 2012;). These coastal environments are the modern marine occurrences marked in figure $4 \mathrm{~A}$ for chlorobactane and okenane, yet they clearly are not representative of the ancient systems we would like to characterize for paleoenvironmental reconstructions. However, there are more comparable examples of unrestricted, transiently sulfidic, marine environments in the modern ocean, such as the Namibian margin (e.g. Weeks et al., 2002). Yet neither okenone nor chlorobactene have been reported in modern transiently sulfidic upwelling zones or even the Black Sea, where isorenieratene has been documented (Repeta et al., 1989). 
The environmental distributions of GSB and PSB carotenoids in modern systems leads to a 'no analog problem' that complicates the interpretation of chlorobactane and okenane in Phanerozoic marine samples. For example, sulfidic chemocline depth estimates based on GSB and PSB pigments in modern lake systems (Brocks and Schaeffer, 2008) may not directly translate to marine water columns because the light quality and controls on mixing are different than in marine systems. Furthermore, the modern environmental distributions of GSB and PSB and their associated carotenoids do not offer support for autochthonous planktonic PSB and GSB production, particularly at shallow depths $(<24 \mathrm{~m})$, in ancient unrestricted marine environments. However, modern observations provide alternative explanations for the occurrence of chlorobactane and okenane in Phanerzoic marine systems, including basin restriction, microbial mat production, and organic matter transport.

GSB and PSB and their associated carotenoids are commonly found in lakes, fjords, lagoons, coastal embayments, and restricted basins (Overmann, 2008). Basin restriction is a common feature of these modern environments, suggesting that basin restriction is an important factor in sustaining a chemocline that is conducive for planktonic GSB and PSB production. Therefore, basin restriction could explain some carotenoid occurrences in the geologic record. The association of basin restriction with modern environments that host planktonic GSB and PSB underscores the importance interpreting the carotenoid biomarkers in the context of robust information about depositional settings as well as physiological and environmental controls on their biosynthesis (Vogl and Bryant, 2012; Smith et al., 2013).

GSB and PSB can live planktonically as well in microbial mats (Overmann, 2008), so microbial mats could be an additional source of GSB and PSB carotenoids in the geologic record. While microbial mats are not always preserved in the rock record, they are associated with some rocks deposited during anoxic events, including some that contain GSB markers (Schouten et al., 2000; Rohl et al., 2001; Gorin et al., 2009; Pacton et 
al., 2009; Marynowski et al., 2011; Kazmierczak et al., 2012). Finally, recent work has highlighted that planktonic and benthic purple sulfur bacteria can have close phylogenetic relationships (Hamilton et al., 2104), further complicating the interpretation of GSB and PSB carotenoid biomarkers in geologic samples.

Lateral advection and transport of organic matter can significantly affect biomarker interpretation. A study based on subfossil $16 \mathrm{~S}$ rRNA discovered that the sequences in Mediterranean sapropels derived more from the freshwater or brackish types of GSB rather than the marine type, and GSB markers were present even in sediment layers deposited under oxic conditions (Coolen and Overmann, 2007). However, these findings remain controversial (Sinninghe Damsté and Hopmans, 2008). Nevertheless, the possibility remains that the $\mathrm{C}_{40}$ carotenoids could have been transported from restricted coastal water columns or sedimentary sources.

The compiled record of marine aromatic $\mathrm{C}_{40}$ carotenoids depicted in figure 4 represents multiple types of depositional environments and potentially a composite of environmental sources (water column vs. sedimentary and autochthonous vs. allochthonous). At present, distinguishing the paleoenvironmental origins of phototrophic sulfur bacterial carotenoids in ancient sedimentary rocks with limited depositional constraints on water column depths and basin geometry remains a challenge that affects the utility of these biomarkers as paleoredox proxies. However, integrating these new data with corresponding patterns of sedimentology, facies, sealevel, paleolatitudes, and biomarkers will further elucidate the source of the GSB and PSB carotenoids in the geologic record.

Sedimentary aromatic $\mathrm{C}_{40}$ carotenoids are found in shales and carbonates (Table 1). They are often associated with particular depositional environments, including ancient lagoons or carbonate platform systems (e.g. Koopmans et al., 1996a; Van KaamPeters and Sinninghe Damsté, 1997; Marynowski et al., 2000; Sepúlveda et al., 2009; Melendez et al., 2013), although not exclusively (French et al, 2014). Furthermore, these 
compounds appear to be common constituents of source rock organic matter and petroleums, suggesting that the environmental conditions conducive for GSB and PSB production are often related to conditions that facilitate the formation of petroleum source rocks. Future work should test whether patterns emerge from the relationship between $\mathrm{C}_{40}$ carotenoids, sedimentology, and facies that could inform the environmental source of these biomarkers.

Additionally, sea level could influence the GSB and PSB carotenoid stratigraphic distribution. Shallow, epeiric seas could promote phototrophic mat growth or facilitate the formation of restricted shallow basins, although the connection between sea level and GSB and PSB production is not well constrained at present. Indeed, chlorobactane and okenane according to the available stratigraphic distribution data appear to be prominent in the Mesozoic, which is an interval that was dominated by epicontinental seas and was a period of prolific source rock formation. However, future studies should test how much of the stratigraphic carotenoid density in the Mesozoic is due to preservation and sampling bias.

Latitudinal deposition affects light intensity in the water column where the high light requirements of planktonic PSB and the green strain of GSB would most likely be accommodated in oligotrophic waters at tropical latitudes. Indeed, chlorobactane has been found in sediments that were deposited between $\sim 0-20^{\circ} \mathrm{N}$ during the end Permian mass extinction (Yin 2005; Hays 2010) and OAE-2 (Kuypers et al., 2002; van Bentum et al., 2009). But low latitudinal deposition is not exclusively characteristic of chlorobactane and okenane occurrence. For example, chlorobactane and okenane were deposited at paleolatitudes $>30^{\circ} \mathrm{N}$ during the end Permian mass extinction and its recovery (Hays et al., 2007; Saito et al., 2014) and the Toarcian OAE (Bassoullet et al., 1993; Schouten et al., 2000; French et al., 2014). Paleolatitude should be considered in the interpretation of GSB and PSB carotenoids in future work, yet the precise relationship between latitudinal deposition and aromatic $\mathrm{C}_{40}$ carotenoids is not yet fully known. 
Finally, integrating the aromatic $\mathrm{C}_{40}$ carotenoid data with complementary biomarkers could also inform the source of the GSB and PSB biomarkers. For example, many samples containing aromatic $\mathrm{C}_{40}$ carotenoids also contain other potentially informative hydrocarbons, such as $\beta$-carotane (e.g. this study), gammacerane (e.g. Cao et al., 2009; Grosjean et al., 2009; Sepulveda et al. 2009; Kelly et al. 2011; Marynowski et al., 2011; French et al., 2014), monomethylalkanes (e.g. Grosjean et al., 2009; Sepúlveda et al. 2009; Kelly et al. 2011; Marynowski et al., 2011). Together, these biomarkers and perhaps others could be used to better constrain the role of basin restriction or mats in samples that contain GSB and PSB carotenoids. However, future work is needed to evaluate the precise relationship between GSB and PSB carotenoids and other potentially informative biomarker compounds.

\subsection{CONCLUSIONS}

Our study has established the utility of tandem mass spectrometry for the detection of saturated and aromatic $\mathrm{C}_{40}$ carotenoid carbon skeletons. The application of these methods illustrates that temporal and paleoenvironmental distributions of aromatic $\mathrm{C}_{40}$ carotenoids in marine systems have been understudied. The revised secular distributions of these compounds may signify more frequent shallow marine PZE $(<24 \mathrm{~m})$ than was previously thought, particularly in the Phanerozoic. However, modern analogs for planktonic production of okenone and chlorobactene are lacking in unrestricted marine environments. While it is possible that planktonic GSB and PSB thrived in unrestricted water columns in past oceans but not in the present ocean, there are alternative explanations for the occurrence of GSB and PSB carotenoids in the geologic record that are supported by contemporary environments, including basin restriction, microbial mats, and allochthonous transport. There is no reason to expect that these factors, which consistently characterize GSB and PSB production and sedimentary archives today, would not have operated in ancient systems. Additionally, 
improved understanding of the physiological and biosynthetic capacities of natural populations of GSB and PSB would strengthen paleoenvironmental interpretations of their biomarkers. The challenge going forward is to distinguish autochthonous water column sources that provide information about global environmental change from other sources of phototrophic sulfur bacterial carotenoids, particularly when depositional environmental information is limited or conflicting.

\subsection{ACKNOWLEDGEMENTS}

We thank Carolyn Colonero for laboratory assistance, Phillipe Schaeffer for the synthetic aromatic carotenoid standards, and Donald Bryant for providing Chlorobium tepidum biomass. We thank Emma Grosjean, Gordon Love, Amy Kelly, and Lindsay Hays for contributing samples to this study. Funding support was provided by grants from the NASA Astrobiology Institute and the NASA Exobiology program to R.E.S and an NSF graduate fellowship to K.L.F.

\subsection{REFERENCES}

Abboud M, Philp RP, Allen J (2005) Geochemical correlation of oils and source rocks from Central and NE Syrian. Journal of Petroleum Geology, 28, 203-216.

Abeed Q, Alkhafaji A, Littke R (2011) Source Rock Potential of the Upper Jurassic-Lower Cretaceous succession in the Southern Mesopotamian Basin, Southern Iraq. Journal of Petroleum Geology, 34, 117-134.

Abeed Q, Leythaeuser D, Littke R (2012) Geochemistry, origin and correlation of crude oils in Lower Cretaceous sedimentary sequences of the southern Mesopotamian Basin, southern Iraq. Organic Geochemistry, 46, 113-126.

Al-Ameri TK, Najaf AA, Al-Khafaji AS, Zumberge J, Pitman J (2013) Hydrocarbon potential of the Sargelu Formation, North Iraq. Arabian Journal of Geosciences, 7, 987-1000.

Amthor J E, Ramseyer K, Faulkner T, Lucas P (2005) Stratigraphy and sedimentology of a chert reservior at the Precambrian-Cambrian Boundary: the Al Shomou Silicilyte, South Oman 
Salt Basin. GeoArabia, 19, 89-122.

Bassoullet JP, Elmi S, Poisson A, Cecca F, Bellion Y, Guiraud R, Baudin F (1993) Mid Toarcian, In: Dercourt J, Ricou LE, Vrielynck B (Eds.) Atlas Tethys Paleoenvironmental Maps, Beicip Franlab, Rueil-Malmaison, 63-80.

Beatty JT, Overmann J, Lince, MT, Manske AK, Lang AS, Blankenship RE, Van Dover CL, Martinson TA, Plumley FG (2005) An obligately photosynthetic bacterial anaerobe from a deep-sea hydrothermal vent. 102, 9206-9310.

Blumenberg M, Thiel V, Riegel W, Kah LC, Reitner J (2012) Biomarkers of black shales formed by microbial mats, Late Mesoproterozoic (1.1 Ga) Taoudeni Basin, Mauritania. Precambrian Research, 196-197, 113-127.

Boreham CJ, Ambrose GJ (2005) Cambrian petroleum systems in the southern Georgina Basin, Northern Territory, Australia. In: Central Australian Basins Symposium petroleum and minerals potential, Alice Springs, NT., pp. 254-281.

Brocks JJ, Schaeffer P (2008) Okenane, a biomarker for purple sulfur bacteria (Chromatiaceae), and other new carotenoid derivatives from the 1640 Ma Barney Creek Formation. Geochimica et Cosmochimica Acta, 72, 1396-1414.

Brocks JJ, Love GD, Summons RE, Knoll AH, Logan GA, Bowden SA (2005) Biomarker evidence for green and purple sulphur bacteria in a stratified Palaeoproterozoic sea. Nature, 437, 866870.

Bryant D, Liu Z, Li T, Zhao F, Costas AG, Klatt C, Ward D, Frigaard N.-U., Overmann J (2012) Comparative and Functional Genomics of Anoxygenic Green Bacteria from the Taxa Chlorobi, Chloroflexi, and Acidobacteria. In: Functional Genomics and Evolution of Photosynthetic Systems (eds. Burnap, R., Vermaas, W.) Springer Netherlands, pp. 47-102.

Burke CM, Burton HR (1988) Photosynthetic bacteria in meromictic lakes and stratified fjords of the Vestfold Hills, Antarctica. Hydrobiologia, 165, 13-23.

Cao C, Love GD, Hays L, Wang W, Shen S, Summons RE (2009) Biogeochemical evidence for euxinic oceans and ecological disturbance presaging the end-Permian mass extinction event. Earth and Planetary Science Letters, 281, 188-201.

Caumette P, Baulaigue R, Matheron R (1991) Thiocapsa halophila sp. nov., a new halophilic phototrophic purple sulfur bacterium. Archive of Microbiology, 155, 170-176.

Caumette P, Guyoneaud R, Imhoff JF, Süling J, Gorlenko V (2004) Thiocapsa marina sp. nov., a novel, okenone-containing, purple sulfur bacterium isolated from brackish coastal and marine environments. International Journal of Systematic and Evolutionary Microbiology, 54, 1031-1036. 
Coolen MJL, Overmann J (2007) 217 000-year-old DNA sequences of green sulfur bacteria in Mediterranean sapropels and their implications for the reconstruction of the paleoenvironment. Environmental Microbiology, 9, 238-249.

Crick IH, Boreham CJ, Cook AC, Powell TG (1988) Petroleum geology and geochemistry of Middle Proterozoic McArthur Basin, northern Australia, II: assessment of source rock potential. AAPG Bulletin, 72, 1495-1514.

Droste H (1990) Depositional cycles and source rock development in an epeiric intra-platform basin: the Hanifa Formation of the Arabian peninsula. Sedimentary Geology, 69, 281-296.

Edwards DS, Summons RE, Kennard JM, Nicoll RS, Bradshaw J, Bradshaw M, Foster CB, OBrien GW, Zumberge JE (1997) Geochemical characterization of Palaeozoic Petroleum Systems in northwestern Australia. The APPEA Journal, 37, 351-379.

Fox JE, Ahlbrandt TS (2002) Petroleum geology and total petroleum systems of the Widyan Basin and interior platform of Saudi Arabia and Iraq. In: U.S. Geological Survey Bulletin E, pp. 1-26.

French KL, Sepúlveda J, Trabucho-Alexandre J, Gröcke DR, Summons RE (2014) Organic geochemistry of the early Toarcian oceanic anoxic event in Hawsker Bottoms, Yorkshire, England. Earth and Planetary Science Letters, 390, 116-127.

Glickson M, Gibson DL, Philp RP (1985) Organic Matter in Australian Cambrian Oil Shales and Other Lower Palaeozoic Shales. Chemical Geology, 51, 175-191.

Gorin G, Fiet N, Pacton M (2009) Benthic microbial mats: a possible major component of organic matter accumulation in the Lower Aptian oceanic anoxic event. Terra Nova, 21, 21-27.

Grosjean E, Love GD, Stalvies C, Fike DA, Summons RE (2009) Origin of petroleum in the Neoproterozoic-Cambrian South Oman Salt Basin. Organic Geochemistry, 40, 87-110.

Hamilton TL, Bovee RJ, Thiel V, Sattin SR, Mohr W, Schaperdoth I, Vogl K, Gilhooly III WP, Lyons TW, Tomsho LP, Schuster SC, Overmann J, Bryant DA, Pearson A, Macalady JL (2014) Coupled reductive and oxidative sulfur cycling in the phototrophic plate of a meromictic lake. Geobiology,

Hartgers WA, Sinninghe Damsté JS, Requejo AG, Allan J, Hayes JM, Ling Y, Xie TM, Primack J, de Leeuw JW (1994) A molecular and carbon isotopic study towards the origin and diagenetic fate of diaromatic carotenoids. Organic Geochemistry, 22, 703-725.

Hays L (2010) Biogeochemical Proxies for Environmental and Biotic Conditions at the PermianTriassic Boundary: MIT dissertation, p. 3-287.

Hays LE, Beatty TW, Henderson CM, Love GD, Summons RE (2007) Evidence for photic zone 
euxinia through the end-Permian mass extinction in the Panthalassic Ocean (Peace River Basin, Western Canada). Palaeoworld, 16, 39-50.

Hoffmann CF, Foster CB, Powell TG, Summons RE (1987) Hydrocarbon biomarkers from Ordovician sediments and the fossil alga Gloeocapsomorpha prisca Zalessky 1917. Geochemica et Cosmochimica Acta, 51, 2681-2697.

Holte J, Gilson J, Talley L, Roemmich D (2010) Argo Mixed Layers, Scripps Institution of Oceanography/UCSD http://mixedlayer.ucsd.eduso.

Jassim SZ, Al-Gailani M (2006) Hydrocarbons, In: Geology of Iraq, Dolin, Prague and Moravian Museum, Brno, pp. 232-250.

Johnston D, Wolfe-Simon F, Pearson A, Knoll A (2009) Anoxygenic photosynthesis modulated Proterozoic oxygen and sustained Earth's middle age. Proceedings of the National Academy of Sciences of the United States of America, 106, 16925-16929.

Kamali MR (1995) Sedimentology and Petroleum Geochemistry of the Ouldburra Formation, Eastern Officer Basin, Australia. PhD Thesis, University of Adelaide, South Australia.

Katz B (1995a) Geochemistry of the Upper Jurassic Tuwaiq Mountain and Hanifa Formation Petroleum Source Rocks of Eastern Saudia Arabia. In: Petroleum Source Rocks, Springer Berlin Heidelberg, pp. 67-87.

Katz B (1995b) The Albian Kazhdumi Formation of the Dezful Embayment, Iran: One of the most efficient petroleum generating systems. In: Petroleum Source Rocks, pp. 183-207.

Kazmierczak J, Kremer B, Racki G (2012) Late Devonian marine anoxia challenged by benthic cyanobacterial mats. Geobiology, 10, 371-383.

Kelly AE, Love GD, Zumberge JE, Summons RE (2011) Hydrocarbon biomarkers of Neoproterozoic to Lower Cambrian oils from eastern Siberia. Organic Geochemistry, 42, 640654.

Konert G, Afifi AM, Al-Hajri SA, de Groot K, Naim Al AA, Droste HJ (2001) Paleozoic Stratigraphy and Hydrocarbon Habitat of the Arabian Plate. In: Petroleum provinces of the twenty-first century, AAPG Memoir, 74, pp. 483-515.

Koopmans MP, Köster J, van Kaam-Peters HME, Kenig F, Schouten S, Hartgers WA, de Leeuw JW, Sinninghe Damsté JS (1996a) Diagenetic and catagenetic products of isorenieratene: Molecular indicators for photic zone anoxia. Geochimica et Cosmochimica Acta, 60, 4467-4496.

Koopmans MP, Schouten S, Kohnen MEL, Sinninghe Damsté JS (1996b) Restricted utility of aryl isoprenoids as indicators for photic zone anoxia. Geochimica et Cosmochimica Acta, 60, 48734876. 
Kuypers M, Pancost R, Nijenhuis I, Sinninghe Damsté JS (2002) Enhanced productivity led to increased organic carbon burial in the euxinic North Atlantic basin during the late Cenomanian oceanic anoxic event. Paleoceanography, 17, 1051.

Lee C, Brocks JJ (2011) Identification of carotane breakdown products in the 1.64 billion year old Barney Creek Formation, McArthur Basin, northern Australia. Organic Geochemistry, 42, 425430.

Long JA, Trinajstic K (2010) The Late Devonian Gogo Formation Lägerstatte of Western Australia: Exceptional Early Vertebrate Preservation and Diversity. Annual Review of Earth and Planetary Science, 38, 255-279.

Lyons TW, Reinhard CT, Scott C (2009) Redox Redux. Geobiology, 7, 489-494.

Lyons TW, Reinhard CT, Planavsky NJ (2014) The rise of oxygen in Earth's early ocean and atmosphere. Nature, 506, 307-315.

Maresca J, Graham J, Bryant D (2008) The biochemical basis for structural diversity in the carotenoids of chlorophototrophic bacteria. Photosynthesis Research, 97, 121-140.

Marynowski L, Narkiewicz M, Grelowski C (2000) Biomarkers as environmental indicators in a carbonate complex, example from the Middle to Upper Devonian, Holy Cross Mountains, Poland. Sedimentary Geology, 137, 187-212.

Marynowski L, Rakociński M, Borcuch E, Kremer B, Schubert BA, Jahren AH (2011) Molecular and petrographic indicators of redox conditions and bacterial communities after the $\mathrm{F} / \mathrm{F}$ mass extinction (Kowala, Holy Cross Mountains, Poland). Palaeogeography, Palaeoclimatology, Palaeoecology, 306, 1-14.

McKirdy DM, Kantsler AJ, Emmet JK, Aldridge AK (1984) Hydrocarbon genesis and organic facies in Cambrian carbonates of the eastern Officer Basin, South Australia. In: Petroleum Geochemistry and Source Rock Potential of Carbonate Rocks (ed. Palacas JG), American Association of Petroleum Geologists Studies in Geology, vol. 18, pp. 13-31.

Melendez I, Grice K, Trinajstic K, Ladjavardi M, Greenwood P, Thompson K (2013) Biomarkers reveal the role of photic zone euxinia in exceptional fossil preservation: An organic geochemical perspective. Geology, 41, 123-126.

Meyer KM, Kump LR (2008) Oceanic euxinia in Earth history: causes and conseqences. Annual Review of Earth and Planetary Sciences 36, 251-288.

Meyer K, Kump L, Ridgwell A (2008) Biogeochemical controls on photic-zone euxinia during the end-Permian mass extinction. Geology, 36, 747-750.

Meyer KM, Macalady JL, Fulton JM, Kump LR, Schaperdoth I, Freeman KH (2011) Carotenoid 
biomarkers as an imperfect reflection of the anoxygenic phototrophic community in meromictic Fayetteville Green Lake. Geobiology, 9, 321-329.

Mohseni H, Al-Aasm IS (2004) Tempestite deposits on a storm-influenced carbonate ramp: an example from the Pabdeh Formation (Paleogene), Zagros Basin, SW Iran. Journal of Petroleum Geology, 27, 163-178.

Mohseni H, Behbahani R, Khodabakhsh S, Atashmard Z (2011) Depositional environments and trace fossil assemblages in the Pabdeh Formation (Paleogene), Zagros Basin, Iran. Neues Jahrbuch für Geologie und Paläontologie - Abhandlungen, 262, 59-77.

Naeher S, Geraga M, Papatheodorou G, Ferentinos G, Kaberi H, Schubert CJ (2012) Environmental variations in a semi-enclosed embayment (Amvrakikos Gulf, Greece) reconstructions based on benthic foraminifera abundance and lipid biomarker pattern. Biogeosciences, 9, 5081-5094.

Overmann J, Cypionka H, Pfenning N (1992) An extremely low-light-adapted phototrophic sulfur bacterium from the Black Sea. Limnology and Oceanography, 37, 150-155.

Overmann J (2008) Ecology of Phototrophic Sulfur Bacteria. In: Sulfur Metabolism in Phototrophic Organisms (eds. Hell et al.). Springer, pp. 375-396.

Pacton M, Gorin G, Fiet N (2009) Occurrence of photosynthetic microbial mats in a Lower Cretaceous black shale (central Italy): a shallow-water deposit. Facies, 55, 401-419.

Peters KE, Walters CC, Moldowan JM (2005) The Biomarker Guide: Biomarkers and isotopes in petroleum systems and Earth history, 2, Cambridge University Press.

Planavsky NJ, Reinhard CT, Wang X, Thompson D, McGoldrick P, Rainbird RH, Johnson T, Fischer WW, Lyons TW (2014) Low Mid-Proterozoic atmospheric oxygen levels and the delayed rise of animals. Science, 346, 635-638.

Rashid H, Grosjean E (2006) Detecting the source of Heinrich layers: An organic geochemical study. Paleoceanography, 21, p. PA3014.

Repeta DJ, Simpson DJ, Jorgenson BB, Jannasch HW (1989) Evidence for anoxygenic photosynthesis from the distribution of bacteriochlorophylls in the Black Sea. Nature, 342, 69-72.

Requejo AG, Creaney S, Gray NR, Cole KS (1992) Aryl isoprenoids and diaromatic carotenoids in Paleozoic source rocks and oils from the Western Canada and Willston Basins. Organic Geochemistry, 19, 245-264.

Röhl HJ, Schmid-Röhl A, Oschmann W, Frimmel A, Schwark L (2001) The Posidonia Shale (Lower Toarcian) of SW-Germany: a oxygen-depleted ecosystem controlled by sea level and 
palaeoclimate. Palaeogeography, Palaeoclimatology, Palaeoecology, 165, 27-52.

Sadooni FN, Alsharhan AS (2004) Stratigraphy, lithofacies distribution, and petroleum potential of the Triassic strata of the northern Arabian plate. AAPG Bulletin, 88, 515-538.

Saito R, Oba M, Kaiho K, Schaeffer P, Adam P, Takahashi S, Nara FW, Chen Z.-Q., Tong J, Tsuchiya N (2014) Extreme euxinia prior to the Middle Triassic biotic recovery from the latest Permian mass extinction. Organic Geochemistry, 73, 113-122.

Schouten S, van Kaam-Peters HME, Rijpstra WIC, Schoell M, Sinninghe Damsté JS (2000) Effects on an oceanic anoxic event on the stable carbon isotopic composition of Early Toarcian carbon. American Journal of Science, 300, 1-22.

Sepúlveda J, Wendler J, Leider A, Kuss H-J, Summons RE, Hinrichs K-U (2009) Molecular isotopic evidence of environmental and ecological changes across the CenomanianTuronian boundary in the Levant Platform of central Jordon. Organic Geochemistry, 40, 553568.

Sinninghe Damsté JS, Hopmans EC (2008) Does fossil pigment and DNA data from Mediterranean sediments invalidate the use of green sulfur bacterial pigments and their diagenetic derivatives as proxies for the assessment of past photic zone euxinia? Environmental Microbiology, 10, 1392-1399.

Sinninghe Damsté JS, Schouten S (2006) Biological markers for anoxia in the photic zone of the water column. In: The Handbook of Environmental Chemistry. Springer Berlin Heidelberg, pp.127-163.

Smith D, Scott J, Steele A, Cody G, Ohara S, Fogel M (2013) Effects of Metabolism and Physiology on the Production of Okenone and Bacteriochlorophyll a in Purple Sulfur Bacteria. Geomicrobiology Journal, 31, 128-137.

Smittenberg RH, Pancost RD, Hopmans EC, Paetzel M, Sinninghe Damsté JS (2004) A 400-year record of environmental change in an euxinic fjord as revealed by the sedimentary biomarker record. Palaeogeography, Palaeoclimatology, Palaeoecology, 202, 331-35.

Southgate PN, Lambert IB, Donnelly TH, Henry R, Etminan H, Weste G (1989) Depositional environments and diagenesis in Lake Parakeelya: a Cambrian alkaline playa from the Officer Basin, South Australia. Sedimentology, 36, 1091-1112.

Sperling E, Wolock C, Johnston DT, Knoll AH (2013) The global record of local iron geochemical data from Proterozoic through Paleozoic basins In: AGU Fall Meeting Abstracts, 1, 1946.

Summons RE, Powell T (1986) Chlorobiaceae in Palaeozoic seas revealed by biological markers, isotopes and geology. Nature, 319, 763-765. 
Summons RE, Powell TG, Boreham CJ (1988) Petroleum geology and geochemistry of the Middle Proterozoic McArthur Basin, northern Australia: III. Composition of extractable hydrocarbons. Geochimica et Cosmochimica Acta, 52, 1747-1763.

Summons RE, Powell TG (1992) Hydrocarbon composition of the Late Proterozoic oils of the Siberian Platform: Implications for the depositional environment of source rocks. In: Early Organic Evolution Springer Berlin Heidelberg, p. 296-307.

Sylvan JB, Toner BM, Edwards KJ (2012) Life and death of deep-sea vents: bacterial diversity on inactive hydrothermal sulfides. mBio, 3, e00279-11.

Ulmishek GF (2001a) Petroleum Geology and resources of the Baykit High province, East Siberia. In: U.S. Geological Survey Bulletin F.

Ulmishek GF (2001b) Petroleum geology and resources of the Nepa-Botuoba High, AngaraLena Terrace, and Cis-Patom Foredeep, Southeastern Siberian craton, Russia. In: U.S. Geological Survey Bulletin C.

van Bentum EC, Hetzel A, Brumsack H.-J., Forster A, Reichart G.-J., Sinninghe Damsté, JS (2009) Reconstruction of water column anoxia in the equatorial Atlantic during the CenomanianTuronian oceanic anoxic event using biomarker and trace metal proxies. Palaeogeography, Palaeoclimatology, Palaeoecology, 280, 489-498.

Van Kaam-Peters HME, Sinninghe Damsté JS (1997) Characterization of an extremely organic sulphur-rich, 150 Ma old carbonaceous rock: palaeoenvironmental implications. Organic Geochemistry, 27, 371-397.

Vila X, Abella CA (1994) Effects of light quality on the physiology and the ecology of planktonic green sulfur bacteria in lakes. Photosynthesis Research, 41, 53-65.

Vogl K, Bryant DA (2012) Biosynthesis of the biomarker okenone: $\chi$-ring formation. Geobiology, 10, 205-215.

Weeks SJ, Currie B, Bakun A (2002) Satellite imaging: Massive emissions of toxic gas in the Atlantic. Nature, 415, 493-494.

Yin HF, Tong J, Zhang K (2005) A review on the Global Stratotype Section and Point of the Permian-Triassic boundary. Acta Geologica Sinica 79, 715-728. 


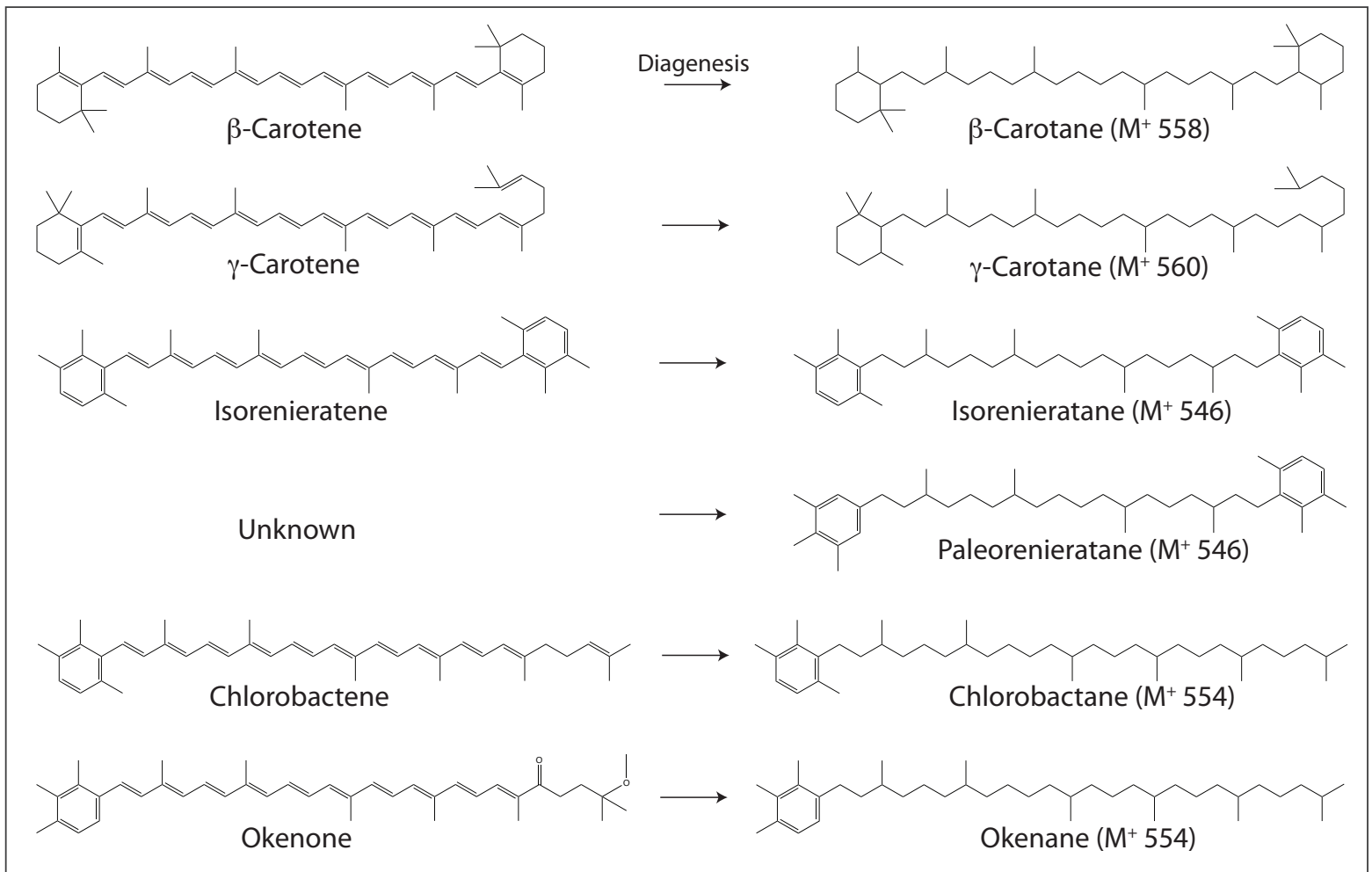

Figure 1. Structures of $\mathrm{C}_{40}$ carotenoids showing the transformation from the biological precursor (left) to the diagenetic product (right). The diagenetic products are labeled with their molecular ion mass to charge ratio. 


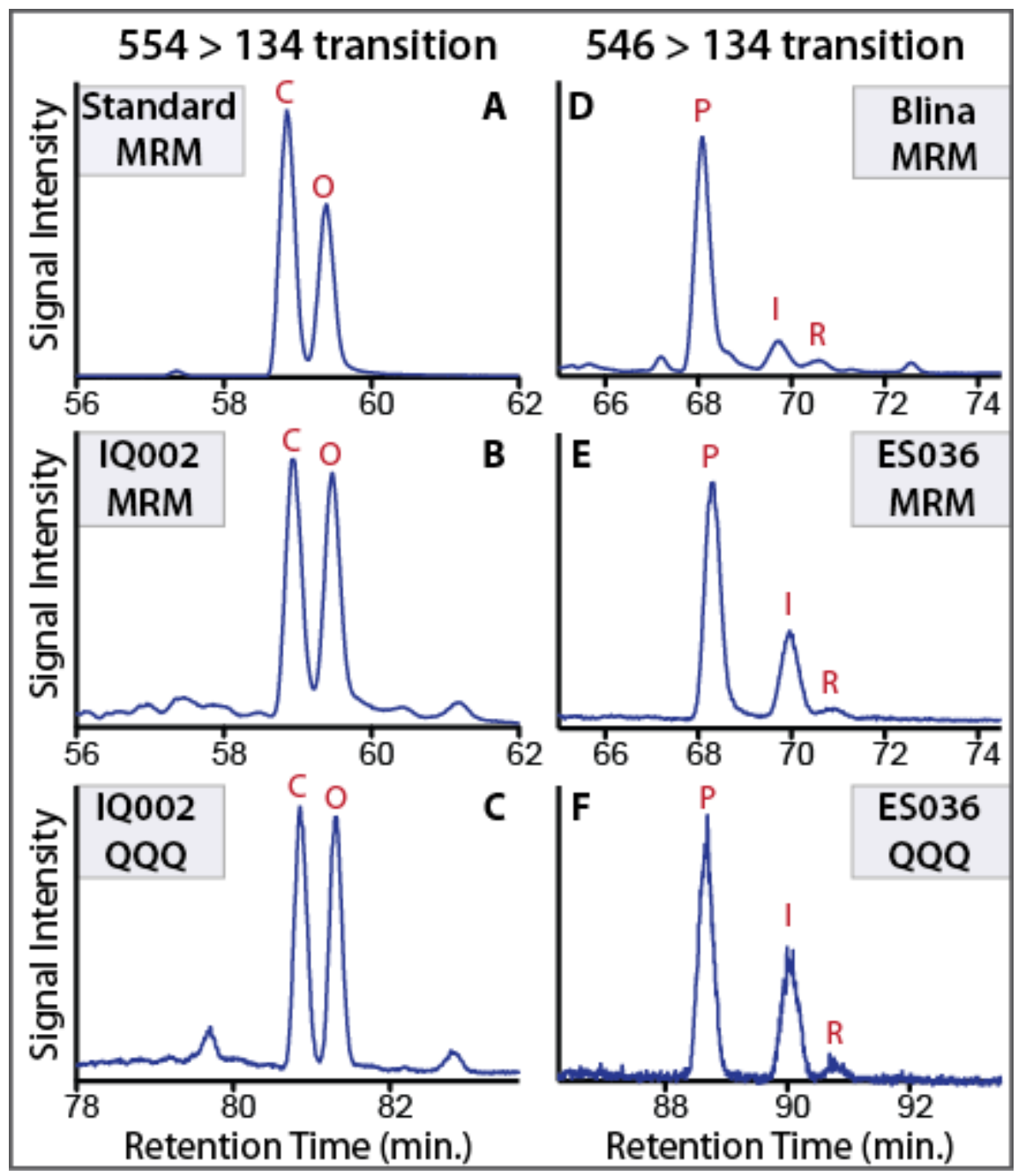

Figure 2. MRM and QQQ chromatograms of aromatic C40 carotenoids identifying chlorobactane $(\mathrm{C})$, okenane $(\mathrm{O})$, paleorenieratane $(\mathrm{P})$, isorenieratane $(\mathrm{I})$, and renieratane (R). Chlorobactane and okenane were detected in a Triassic oil (IQ002) by MRM (B) and QQQ (C). These results are compared to the MRM results of the combined synthetic $\mathrm{C}_{40}$ standard (A). Paleorenieratane, isorenieratane, and renieratane were detected in a Neoproterozoic oil (ES036) by MRM (E) and QQQ (F), and these results are compared to the MRM results of a previously characterized Devonian oil (D). The difference in retention times between the MRM and QQQ results is due to different GC conditions. 


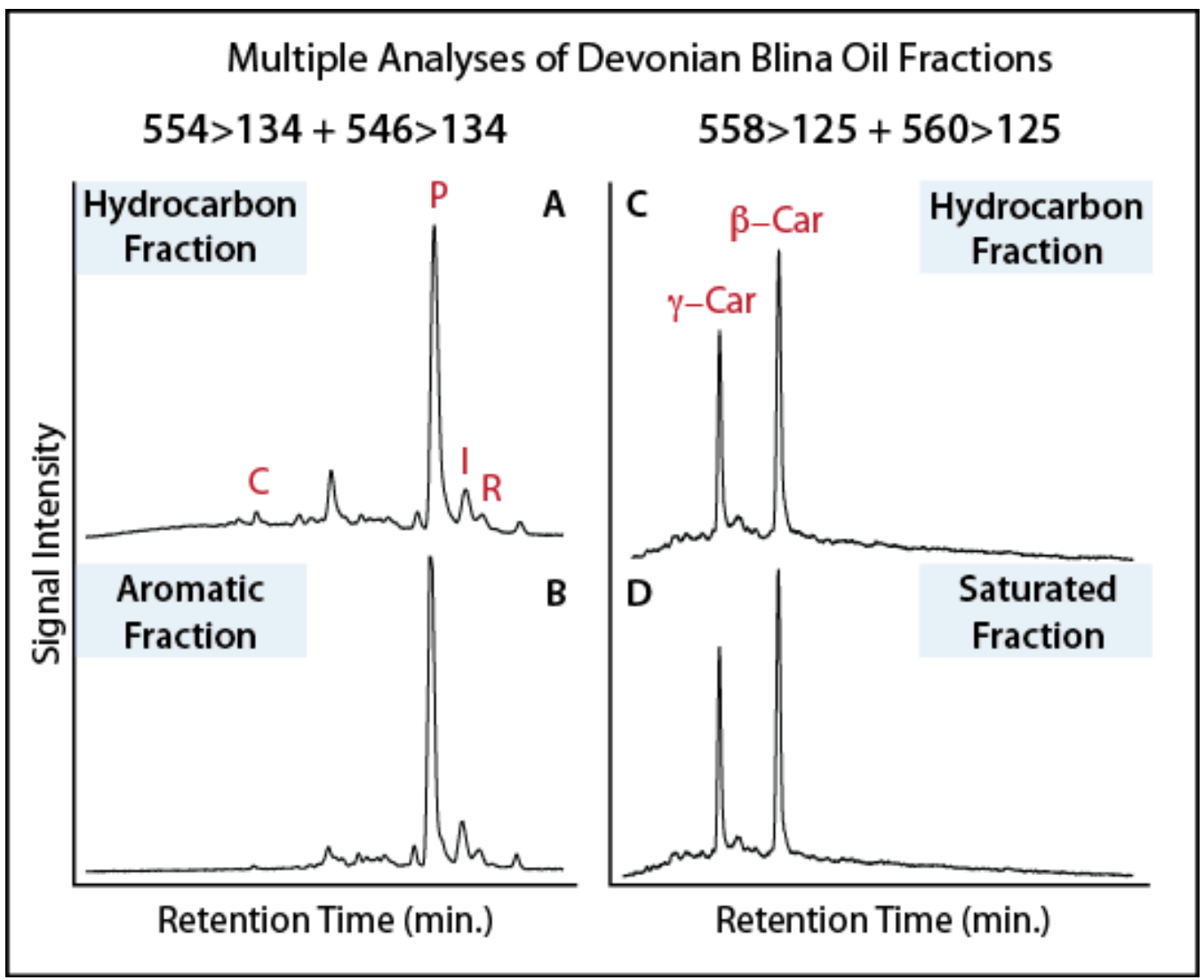

Figure 3. Simultaneous detection of aromatic and saturated sedimentary $C_{40}$ carotenoids by tandem mass spectrometry. Figures A-D show MRM results of Blina oil (Devonian) fractions. Aromatic $\mathrm{C}_{40}$ carotenoids can be detected in hydrocarbon fractions, which include both saturated and aromatic compounds, and aromatic fractions (A and B). Saturated $\mathrm{C}_{40}$ carotanes can be detected in hydrocarbon fractions and saturated fractions (C and D) or branched/cyclic fractions (not shown). Compounds are labeled numerically: 1. Chlorobactane; 2. Paleorenieratane; 3. Isorenieratane; 4. Renieratane; 5. $\gamma$-carotane; 6. $\beta$-carotane 


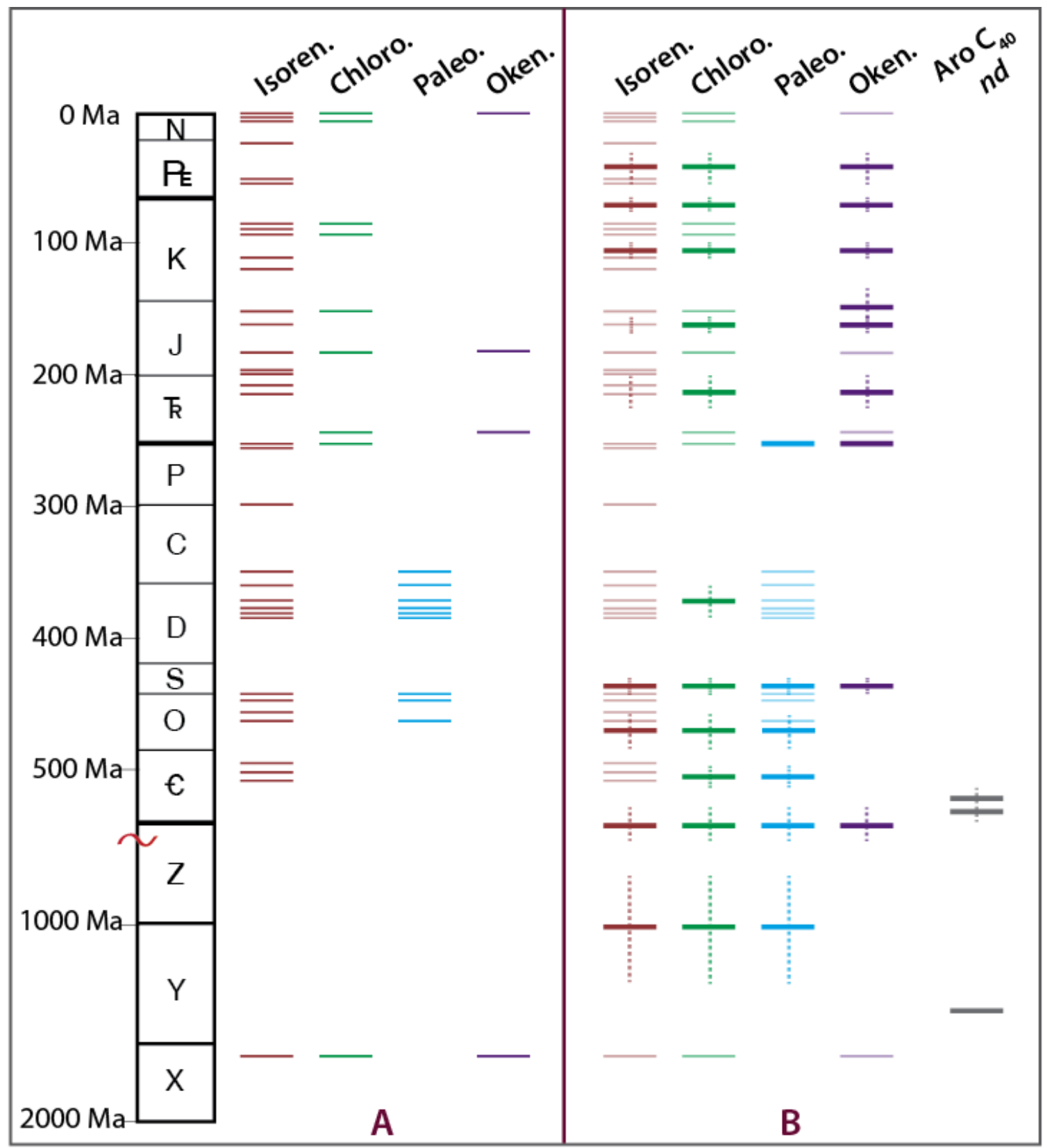

Figure 4. Stratigraphic distribution of aromatic $\mathrm{C}_{40}$ carotenoids according to current literature (A) and our new QQQ and MRM results, where the published results are represented in faded color tones for comparison (B). The dashed lines in panel B represent sample age uncertainty, and a red symbol marks the change of the vertical scale in the Precambrian compared to the Phanerozoic. Isorenieratane, chlorobactane, paleorenieratane, and okenane are brown, green, blue, and purple, respectively. Samples that were analyzed by MRM but did not contain aromatic $\mathrm{C}_{40}$ carotenoids (Aro $\mathrm{C}_{40} n d$ ) are represented in grey in panel $\mathrm{B}$. 
Table 1. MRM and QQQ sample information and $\mathrm{C}_{40}$ carotenoid results

\begin{tabular}{|c|c|c|c|c|c|c|c|c|}
\hline Age & $\begin{array}{c}\mathrm{C}_{40} \\
\text { Carot- } \\
\text { enoids }^{\mathrm{a}} \\
\end{array}$ & Sample ID & Country & Basin/Well & \begin{tabular}{c}
\multicolumn{1}{c}{ Oil } \\
Field/Rock \\
Formation \\
\end{tabular} & $\begin{array}{c}\text { Inferred or } \\
\text { Known Source } \\
\text { and Lithology }\end{array}$ & $\begin{array}{l}\text { Analysis } \\
\text { Type }\end{array}$ & References \\
\hline $\begin{array}{l}\text { Late Paleocene to } \\
\text { Early Oligocene }\end{array}$ & $1,2,4,5$ & IN0093 HC & Iran & Zagros Fold Belt & Qaleh-Nar & $\begin{array}{l}\text { carbonate ramp to } \\
\text { basin; Pabdeh Fm. }\end{array}$ & Oil QQQ & $\begin{array}{l}\text { (Mohseni and Al-Aasm, 2004; } \\
\text { Mohseni et al., 2011) }\end{array}$ \\
\hline Upper Cretaceous & $1,2,4,5$ & SY0058 HC & Syria & Euphrates Graben & Jafra & distal marine shale & Oil QQQ & na \\
\hline $\begin{array}{l}\text { Upper Cretaceous } \\
\text { (Campanian- } \\
\text { Maastrichtian) }\end{array}$ & $1,2,4,5$ & SY0013 HC & Syria & Eastern Syria & Tishreen & $\begin{array}{l}\text { marine marl; } \\
\text { Shiranish Fm. }\end{array}$ & Oil QQQ & $\begin{array}{l}\text { (Abboud et al., 2005; Jassim } \\
\text { and Al-Gailani, 2006) }\end{array}$ \\
\hline $\begin{array}{l}\text { Upper Cretaceous } \\
\text { (Campanian- } \\
\text { Maastrichtian) }\end{array}$ & $1,2,4,5$ & SY0030 HC & Syria & Eastern Syria & Shedadeh & $\begin{array}{l}\text { marine marl; } \\
\text { Shiranish Fm. }\end{array}$ & Oil QQQ & $\begin{array}{l}\text { (Abboud et al., 2005; Jassim } \\
\text { and Al-Gailani, 2006) }\end{array}$ \\
\hline $\begin{array}{l}\text { Upper Cretaceous } \\
\text { (Albian) }\end{array}$ & $1,2,4,5$ & SY0003 HC & Syria & Eastern Syria & Al Hol & $\begin{array}{l}\text { marine carbonate; } \\
\text { Kazhdumi Fm. }\end{array}$ & Oil QQQ & $\begin{array}{l}\text { (Katz, 1995b; Peters et al., } \\
\text { 2005) }\end{array}$ \\
\hline $\begin{array}{l}\text { Lower Cretaceous } \\
\text { (Albian) }\end{array}$ & $1,2,5$ & IQ0010 HC & Iraq & Zagros Fold Belt & Injana & $\begin{array}{l}\text { marine marl; } \\
\text { Kazhdumi Fm. }\end{array}$ & Oil QQQ & $\begin{array}{l}\text { (Katz, 1995b; Peters et al., } \\
\text { 2005) }\end{array}$ \\
\hline $\begin{array}{l}\text { Lower Cretaceous } \\
\text { (Albian) }\end{array}$ & $1,2,4,5$ & IN0101 HC & Iran & Dibdibah Sub-basin & Darguin & $\begin{array}{l}\text { marine carbonate; } \\
\text { Kazhdumi Fm. }\end{array}$ & Oil QQQ & $\begin{array}{l}\text { (Katz, 1995b; Peters et al., } \\
\text { 2005) }\end{array}$ \\
\hline Lower Cretaceous & $1,2,5$ & IN0061 HC & Iran & Dibdibah Sub-basin & Azedegan & marine marl & Oil QQQ & na \\
\hline Lower Cretaceous & $1,2,5$ & IN0062 HC & Iran & Dibdibah Sub-basin & Darguin & marine marl & Oil QQQ & na \\
\hline $\begin{array}{l}\text { Upper Jurassic } \\
\text { (Kimmeridgian- } \\
\text { Oxfordian) }\end{array}$ & $1,2,5$ & IN0104 HC & Iran & Dibdibah Sub-basin & Azadegan & $\begin{array}{l}\text { marine carbonate; } \\
\text { Naokelekan Fm.? }\end{array}$ & Oil QQQ & (Abeed et al., 2011) \\
\hline $\begin{array}{l}\text { Upper Jurassic } \\
\text { (Kimmeridgian- } \\
\text { Oxfordian) }\end{array}$ & $1,2,4,5$ & IQ0034 HC & Iraq & Zagros Fold Belt & Kirkuk & $\begin{array}{l}\text { marine carbonate; } \\
\text { Naokelekan Fm.? }\end{array}$ & Oil QQQ & (Abeed et al., 2011) \\
\hline $\begin{array}{l}\text { Upper Jurassic } \\
\text { (Oxfordian) }\end{array}$ & $1,2,4,5$ & SY0005 HC & Syria & Eastern Syria & Leilak & $\begin{array}{l}\text { marine carbonate; } \\
\text { Hanifa Fm. \& } \\
\text { equivalents }\end{array}$ & Oil QQQ & $\begin{array}{l}\text { (Droste, 1990; Peters et al., } \\
\text { 2005; Katz, 1995a) }\end{array}$ \\
\hline $\begin{array}{l}\text { Upper Jurassic } \\
\text { (Oxfordian) }\end{array}$ & $1,2,4,5$ & TK0019 HC & Turkey & Selmo-Raman & Raman & $\begin{array}{l}\text { marine carbonate; } \\
\text { Hanifa Fm. \& } \\
\text { equivalents }\end{array}$ & Oil QQQ & $\begin{array}{l}\text { (Katz, 1995a; Peters et al., } \\
\text { 2005) }\end{array}$ \\
\hline $\begin{array}{l}\text { Upper Jurassic } \\
\text { (Oxfordian) }\end{array}$ & $1,2,4,5$ & TK0063 HC & Turkey & Diyarbakir & Magrip & $\begin{array}{l}\text { marine carbonate; } \\
\text { Hanifa Fm. \& } \\
\text { equivalents }\end{array}$ & Oil QQQ & $\begin{array}{l}\text { (Katz, 1995a; Peters et al., } \\
\text { 2005) }\end{array}$ \\
\hline $\begin{array}{l}\text { Upper Jurassic } \\
\text { (Oxfordian) }\end{array}$ & $1,2,4,5$ & SY0067 HC & Syria & Eastern Syria & Rumelan & $\begin{array}{l}\text { marine carbonate; } \\
\text { Hanifa Fm. \& } \\
\text { equivalents }\end{array}$ & Oil QQQ & $\begin{array}{l}\text { (Katz, 1995a; Peters et al., } \\
\text { 2005) }\end{array}$ \\
\hline
\end{tabular}




\begin{tabular}{|c|c|c|c|c|c|c|c|c|}
\hline $\begin{array}{l}\text { Upper Jurassic } \\
\text { (Oxfordian) }\end{array}$ & $1,2,4,5$ & KW0004 HC & Kuwait & $\begin{array}{l}\text { Burgan Rumaila } \\
\text { High }\end{array}$ & Minagish & $\begin{array}{l}\text { marine carbonate; } \\
\text { Hanifa Fm. \& } \\
\text { equivalents }\end{array}$ & Oil QQQ & $\begin{array}{l}\text { (Droste, 1990; Peters et al., } \\
\text { 2005; Katz, 1995a) }\end{array}$ \\
\hline $\begin{array}{l}\text { Upper Jurassic } \\
\text { (Oxfordian) }\end{array}$ & $1,2,5$ & SA0035 HC & $\begin{array}{l}\text { Saudi } \\
\text { Arabia }\end{array}$ & Summan Platform & Rimthan & $\begin{array}{l}\text { marine carbonate; } \\
\text { Hanifa Fm. \& } \\
\text { equivalents }\end{array}$ & Oil QQQ & $\begin{array}{l}\text { (Droste, 1990; Peters et al., } \\
\text { 2005; Katz, 1995a) }\end{array}$ \\
\hline $\begin{array}{l}\text { Upper Jurassic } \\
\text { (Late Callovian- } \\
\text { Oxfordian) }\end{array}$ & $1,2,4,5$ & IQ0016 HC & Iraq & $\begin{array}{l}\text { Burgan Rumaila } \\
\text { High }\end{array}$ & Tuba & $\begin{array}{l}\text { marine carbonate; } \\
\text { Najmah Fm. }\end{array}$ & Oil QQQ & $\begin{array}{l}\text { (Fox and Ahlbrandt, 2002; } \\
\text { Peters et al., 2005) }\end{array}$ \\
\hline $\begin{array}{l}\text { Upper Jurassic } \\
\text { (Late Callovian- } \\
\text { Oxfordian) }\end{array}$ & $1,2,4,5$ & IQ0018 HC & Iraq & $\begin{array}{l}\text { Burgan Rumaila } \\
\text { High }\end{array}$ & Tuba & $\begin{array}{l}\text { marine carbonate; } \\
\text { Najmah Fm. }\end{array}$ & Oil QQQ & $\begin{array}{l}\text { (Fox and Ahlbrandt, 2002; } \\
\text { Peters et al., 2005) }\end{array}$ \\
\hline $\begin{array}{l}\text { Middle Jurassic } \\
\text { (Bajocian- } \\
\text { Callovian) }\end{array}$ & $1,2,4,5$ & IQ0089 HC & Iraq & Southern Iraq & West Qurna & $\begin{array}{l}\text { marine carbonate; } \\
\text { Sargelu Fm. }\end{array}$ & Oil QQQ & $\begin{array}{l}\text { (Jassim and Al-Gailani, 2006; } \\
\text { Abeed et al., 2012; Peters et } \\
\text { al., 2005) }\end{array}$ \\
\hline $\begin{array}{l}\text { Middle Jurassic } \\
\text { (Bajocian- } \\
\text { Callovian) }\end{array}$ & $1,2,4,5$ & IQ0091 HC & Iraq & Southern Iraq & West Qurna & $\begin{array}{l}\text { marine carbonate; } \\
\text { Sargelu Fm. }\end{array}$ & Oil QQQ & $\begin{array}{l}\text { (Jassim and Al-Gailani, 2006; } \\
\text { Abeed et al., 2012; Peters et } \\
\text { al., 2005) }\end{array}$ \\
\hline $\begin{array}{l}\text { Middle Jurassic } \\
\text { (Bajocian- } \\
\text { Callovian) }\end{array}$ & $1,2,4,5$ & IQ0013 HC & Iraq & $\begin{array}{l}\text { Burgan Rumaila } \\
\text { High }\end{array}$ & Rachi & $\begin{array}{l}\text { marine carbonate; } \\
\text { Sargelu Fm. }\end{array}$ & Oil QQQ & $\begin{array}{l}\text { (Jassim and Al-Gailani, 2006; } \\
\text { Abeed et al., 2012; Peters et } \\
\text { al., 2005) }\end{array}$ \\
\hline $\begin{array}{l}\text { Middle Jurassic } \\
\text { (Bajocian- } \\
\text { Callovian) }\end{array}$ & $1,2,5$ & IQ0020 HC & Iraq & Zagros Fold Belt & Kirkuk & $\begin{array}{l}\text { marine carbonate; } \\
\text { Sargelu Fm. }\end{array}$ & Oil QQQ & $\begin{array}{l}\text { (Jassim and Al-Gailani, 2006; } \\
\text { Abeed et al., 2012; Al-Ameri } \\
\text { et al., 2013; Peters et al., } \\
\text { 2005) }\end{array}$ \\
\hline $\begin{array}{l}\text { Middle Jurassic } \\
\text { (Bajocian- } \\
\text { Callovian) }\end{array}$ & $1,2,5$ & IQ0064 HC & Iraq & $\begin{array}{l}\text { Mesopotamian } \\
\text { Foredeep Basin }\end{array}$ & $\begin{array}{l}\text { East } \\
\text { Baghdad } \\
\text { field }\end{array}$ & $\begin{array}{l}\text { marine carbonate; } \\
\text { Sargelu Fm. }\end{array}$ & Oil QQQ & $\begin{array}{l}\text { (Jassim and Al-Gailani, 2006; } \\
\text { Abeed et al., 2012; Peters et } \\
\text { al., 2005) }\end{array}$ \\
\hline Late Triassic & $1,2,4,5,6$ & $\begin{array}{l}\text { SY0066 HC, } \\
\text { Aros, } \mathrm{Br} / \mathrm{Cy}\end{array}$ & Syria & $\begin{array}{l}\text { Euphrates/ } \\
\text { Mardin }\end{array}$ & Oudeh & $\begin{array}{l}\text { marine carbonate; } \\
\text { Kurra Chine }\end{array}$ & $\begin{array}{l}\text { Oil QQQ } \\
\& \text { MRM }\end{array}$ & $\begin{array}{l}\text { (Abboud et al., 2005; Sadooni } \\
\text { and Alsharhan, 2004) }\end{array}$ \\
\hline Late Triassic & $1,2,4,5,6$ & $\begin{array}{l}\text { IQ0002 HC, } \\
\text { Aros, } \mathrm{Br} / \mathrm{Cy}\end{array}$ & Iraq & Zagros Fold Belt & Alan & $\begin{array}{l}\text { marine carbonate; } \\
\text { Kurra Chine }\end{array}$ & $\begin{array}{l}\text { Oil QQQ } \\
\& \text { MRM }\end{array}$ & $\begin{array}{l}\text { (Abboud et al., 2005; Sadooni } \\
\text { and Alsharhan, 2004) }\end{array}$ \\
\hline Early Triassic (PT) & $1,2,3$ & $\begin{array}{l}\text { G2-1a, 2a } \\
\text { Aros }\end{array}$ & $\begin{array}{l}\text { Western } \\
\text { Canada }\end{array}$ & Peace River & na & $\begin{array}{l}\text { silty mudstone to } \\
\text { muddy sandstone }\end{array}$ & $\begin{array}{l}\text { Rock } \\
\text { MRM }\end{array}$ & $\begin{array}{l}\text { (Hays, 2010; Hays et al., } \\
\text { 2007) }\end{array}$ \\
\hline Early Triassic (PT) & $1,2,4$ & $\begin{array}{l}\text { G2-2b } \\
\text { Aros }\end{array}$ & $\begin{array}{l}\text { Western } \\
\text { Canada }\end{array}$ & Peace River & na & $\begin{array}{l}\text { silty mudstone to } \\
\text { muddy sandstone }\end{array}$ & $\begin{array}{l}\text { Rock } \\
\text { MRM }\end{array}$ & $\begin{array}{l}\text { (Hays, 2010; Hays et al., } \\
\text { 2007) }\end{array}$ \\
\hline Upper Devonian & $1,2,3,5,6$ & $\begin{array}{l}\text { Blina-1, Blina } \\
19999113 \text { HC, } \\
\text { Sats, Aros }\end{array}$ & Australia & Canning (Blina) & na & marine shale & $\begin{array}{l}\text { Rock } \\
\text { MRM }\end{array}$ & $\begin{array}{l}\text { (Long and Trinajstic, 2010; } \\
\text { Edwards et al., 1997) }\end{array}$ \\
\hline Lower Silurian & $1,2,3,5$ & TK0004 HC & $\begin{array}{l}\text { Syria/ } \\
\text { Turkey }\end{array}$ & Kurkan-Karakoy & $\begin{array}{l}\text { Sahaban } \\
\text { South }\end{array}$ & $\begin{array}{l}\text { distal marine shale; } \\
\text { Mudawwara/ } \\
\text { Qusaiba }\end{array}$ & Oil QQQ & (Konert et al., 2001) \\
\hline
\end{tabular}




\begin{tabular}{|c|c|c|c|c|c|c|c|c|}
\hline Lower Silurian & $1,2,3,4,5$ & TK0016 HC & $\begin{array}{l}\text { Syria/ } \\
\text { Turkey }\end{array}$ & Kurkan-Karakoy & Beykan & $\begin{array}{l}\text { distal marine shale; } \\
\text { Mudawwara/ } \\
\text { Qusaiba }\end{array}$ & Oil QQQ & (Konert et al., 2001) \\
\hline Lower Silurian & $1,2,3,4,5$ & TK0017 HC & $\begin{array}{l}\text { Syria/ } \\
\text { Turkey }\end{array}$ & Kurkan-Karakoy & Barbes & $\begin{array}{l}\text { distal marine shale; } \\
\text { Mudawwara/ } \\
\text { Qusaiba }\end{array}$ & Oil QQQ & (Konert et al., 2001) \\
\hline $\begin{array}{l}\text { Early-Middle } \\
\text { Ordovician }\end{array}$ & $1,2,3,5,6$ & $\begin{array}{l}\text { AGSO-299 HC, } \\
\text { Aros, Sats }\end{array}$ & Australia & Canning (Dodonea-1) & Goldwyer & marine shale & Oil MRM & (Hoffmann et al., 1987) \\
\hline Middle Cambrian & $1,2,3$ & $\begin{array}{l}\text { AGSO } 1316 \\
\text { Aros }\end{array}$ & Australia & Georgina (Mt Isa-1) & $\begin{array}{l}\text { Currant } \\
\text { Bush }\end{array}$ & marine carbonate & $\begin{array}{l}\text { Rock } \\
\text { MRM }\end{array}$ & $\begin{array}{l}\text { (Boreham and Ambrose, } \\
\text { 2005; Glickson et al., 1985) }\end{array}$ \\
\hline Middle Cambrian & $1,2,3$ & $\begin{array}{l}\text { AGSO } 1361 \\
\text { Aros }\end{array}$ & Australia & Georgina (Mt Isa-1) & Inca & marine carbonate & $\begin{array}{l}\text { Rock } \\
\text { MRM }\end{array}$ & $\begin{array}{l}\text { (Boreham and Ambrose, } \\
\text { 2005) }\end{array}$ \\
\hline Middle Cambrian & 1,2 & $\begin{array}{l}\text { AGSO } 5679 \\
\text { Aros }\end{array}$ & Australia & Georgina (Owen-2) & Arthur Ck. & marine 'hot shale' & $\begin{array}{l}\text { Rock } \\
\text { MRM }\end{array}$ & $\begin{array}{l}\text { (Boreham and Ambrose, } \\
\text { 2005) }\end{array}$ \\
\hline Middle Cambrian & 1,3 & $\begin{array}{l}\text { AGSO } 5683 \\
\text { Aros }\end{array}$ & Australia & Georgina (Owen-2) & $\begin{array}{l}\text { Thorntonia } \\
\text { Limestone }\end{array}$ & marine carbonate & $\begin{array}{l}\text { Rock } \\
\text { MRM }\end{array}$ & $\begin{array}{l}\text { (Boreham and Ambrose, } \\
\text { 2005) }\end{array}$ \\
\hline $\begin{array}{l}\text { Early-Middle } \\
\text { Cambrian }\end{array}$ & $\begin{array}{l}5, \text { Aro } \mathrm{C}_{40} \\
\text { nd }\end{array}$ & $\begin{array}{l}\text { AGSO 2357, } \\
2358,2359 \text { Sats, } \\
\text { Aros }\end{array}$ & $\begin{array}{l}\text { South } \\
\text { Australia }\end{array}$ & $\begin{array}{l}\text { Officer (Byilkaoora- } \\
\text { 3) }\end{array}$ & $\begin{array}{l}\text { Observatory } \\
\text { Hill Fm. }\end{array}$ & $\begin{array}{l}\text { Parakeelya Alkali } \\
\text { Member; alkaline } \\
\text { playa lake }\end{array}$ & $\begin{array}{l}\text { Rock } \\
\text { MRM }\end{array}$ & $\begin{array}{l}\text { (McKirdy et al., 1984; } \\
\text { Southgate et al., 1989) }\end{array}$ \\
\hline Early Cambrian & Aro $\mathrm{C}_{40} \mathrm{nd}$ & $\begin{array}{l}\text { AGSO } 3499 \\
\text { Aros }\end{array}$ & Australia & $\begin{array}{l}\text { Officer } \\
\text { (Marla-3) }\end{array}$ & $\begin{array}{l}\text { Ouldburra } \\
\text { Fm. }\end{array}$ & marine marl & $\begin{array}{l}\text { Rock } \\
\text { MRM }\end{array}$ & $\begin{array}{l}\text { (McKirdy et al., 1984; } \\
\text { Kamali, 1995) }\end{array}$ \\
\hline $\begin{array}{l}\text { Ediacaran-Early } \\
\text { Cambrian }\end{array}$ & 2,4 & OMR004 Aros & Oman & At-1 well & $\begin{array}{l}\text { Ara Group } \\
\text { A4 Athel } \\
\text { Silicylite }\end{array}$ & $\begin{array}{l}\text { basinal marine } \\
\text { organic rich micro- } \\
\text { crystalline silica }\end{array}$ & $\begin{array}{l}\text { Rock } \\
\text { MRM }\end{array}$ & $\begin{array}{l}\text { (Grosjean et al., 2009; Amthor } \\
\text { et al., 2005) }\end{array}$ \\
\hline $\begin{array}{l}\text { Ediacaran-Early } \\
\text { Cambrian }\end{array}$ & 2,4 & OMR012 Aros & Oman & Ma-1 well & $\begin{array}{l}\text { Ara Group } \\
\text { A4 Athel } \\
\text { Silicylite }\end{array}$ & $\begin{array}{l}\text { basinal marine } \\
\text { organic rich micro- } \\
\text { crystalline silica }\end{array}$ & $\begin{array}{l}\text { Rock } \\
\text { MRM }\end{array}$ & $\begin{array}{l}\text { (Grosjean et al., 2009; Amthor } \\
\text { et al., 2005) }\end{array}$ \\
\hline $\begin{array}{l}\text { Ediacaran-Early } \\
\text { Cambrian }\end{array}$ & 2,4 & OMR013 Aros & Oman & Ma-1 well & $\begin{array}{l}\text { Ara Group } \\
\text { A4 Athel } \\
\text { Silicylite }\end{array}$ & $\begin{array}{l}\text { basinal marine } \\
\text { organic rich micro- } \\
\text { crystalline silica }\end{array}$ & $\begin{array}{l}\text { Rock } \\
\text { MRM }\end{array}$ & $\begin{array}{l}\text { (Grosjean et al., 2009; Amthor } \\
\text { et al., 2005) }\end{array}$ \\
\hline $\begin{array}{l}\text { Late Ediacaran- } \\
\text { Early Cambrian }\end{array}$ & $1,2,3,5,6$ & $\begin{array}{l}\text { ES083 HC, } \\
\text { Aros, Br/Cy }\end{array}$ & $\begin{array}{l}\text { Eastern } \\
\text { Siberia }\end{array}$ & Nepa-Botuoba & Sanar & $\begin{array}{l}\text { marine marl; } \\
\text { Usol'ye Fm. }\end{array}$ & $\begin{array}{l}\text { Oil QQQ } \\
\& \text { MRM }\end{array}$ & $\begin{array}{l}\text { (Kelly et al., 2011; Ulmishek, } \\
\text { 2001b; Ulmishek, 2001a) }\end{array}$ \\
\hline $\begin{array}{l}\text { Late Ediacaran- } \\
\text { Early Cambrian }\end{array}$ & $1,2,3,5,6$ & $\begin{array}{l}\text { ES036 HC, } \\
\text { Aros, } \mathrm{Br} / \mathrm{Cy}\end{array}$ & $\begin{array}{l}\text { Eastern } \\
\text { Siberia }\end{array}$ & Nepa-Botuoba & Yaraktin & $\begin{array}{l}\text { marine marl; Nepa } \\
\text { Fm. }\end{array}$ & $\begin{array}{l}\text { Oil QQQ } \\
\& \text { MRM }\end{array}$ & $\begin{array}{l}\text { (Kelly et al., 2011; Ulmishek, } \\
\text { 2001b; Ulmishek, 2001a) }\end{array}$ \\
\hline Riphean & $1,2,3,5,6$ & $\begin{array}{l}\text { ES020 HC, } \\
\text { Aros, } \mathrm{Br} / \mathrm{Cy}\end{array}$ & $\begin{array}{l}\text { Eastern } \\
\text { Siberia }\end{array}$ & Baykit High & Yurubchen & $\begin{array}{l}\text { marine marl; } \\
\text { Kamov group }\end{array}$ & $\begin{array}{l}\text { Oil QQQ } \\
\& \text { MRM }\end{array}$ & $\begin{array}{l}\text { (Kelly et al., 2011; Ulmishek, } \\
\text { 2001b; Ulmishek, 2001a) }\end{array}$ \\
\hline Mesoproterozoic & Aro $\mathrm{C}_{40}$ nd & $\begin{array}{l}\text { Urapunga-3, } \\
24.5 \mathrm{~m} \text { Aros }\end{array}$ & Australia & Urapunga-3 & Velkerri Fm. & marine shale & $\begin{array}{l}\text { Rock } \\
\text { MRM }\end{array}$ & $\begin{array}{l}\text { (Crick et al., 1988; Summons } \\
\text { et al., 1988) }\end{array}$ \\
\hline
\end{tabular}

${ }^{\mathrm{b}}$ HC: hydrocarbon fraction; Sats: Saturated fraction; Br/Cy: Branched/Cyclic fraction; Aros: aromatic fraction 


\subsection{SUPPLEMENTARY ONLINE MATERIAL:}

\subsubsection{Analytical conditions for GC-MRM-MS and GC-QQQ-MS:}

The Waters Micromass Autospec Ultima was equipped with an Agilent 6890N gas chromatograph and a J\&W Scientific DB-5 fused silica capillary column (60 m x 0.25 $\mathrm{mm}$ i.d., $0.25 \mu \mathrm{m}$ film thickness) using He as carrier gas. The GC oven was ramped from $60^{\circ} \mathrm{C}\left(1 \mathrm{~min}\right.$.) to $155^{\circ} \mathrm{C}$ at $15^{\circ} \mathrm{C} / \mathrm{min}$ and then to $325^{\circ} \mathrm{C}$ at $4^{\circ} \mathrm{C} / \mathrm{min}$, finally holding for $25.20 \mathrm{~min}$. Samples were injected in splitless mode into a PTV injector at $330^{\circ} \mathrm{C}$. The perfluorokerosene (PFK) tune file was modified to include a lower magnet mass from an air peak in order to improve analysis of the intact $\mathrm{C}_{40}$ carotenoid carbon skeletons. The MS source was operated in EI-mode at $250^{\circ} \mathrm{C}$ with an ionization energy of $70 \mathrm{eV}$ and an $8 \mathrm{kV}$ acceleration voltage. The molecular ion to fragment ion transitions were acquired as two consecutive functions. The first function comprised transitions corresponding to the $\mathrm{C}_{14-25}$ aryl isoprenoids, and the second function monitored 13 transitions including the $\mathrm{C}_{40}$ carotenoid transitions, which had a total cycle time of $819.76 \mathrm{~ms}$. Due to the different intensities of the $\mathrm{m} / \mathrm{z} 133 / 134$ base peak intensities, the 546 => 134 transition is enhanced for paleorenieratane relative to isorenieratane. With the exception of paleorenieratane and $\gamma$-carotane, compound identification for the MRM analysis was confirmed by co-elution experiments with synthetic standards and a rock extract from the BCF (Brocks et al., 2005). The identification of paleorenieratane for MRM analysis was confirmed by co-elution experiments with a Devonian sample, Blina-1, from Western Australia containing paleorenieratane and by comparison of a full scan mass spectrum with published mass spectra (Requejo et al., 1992).

The GC-QQQ-MS was operated in multiple reaction monitoring mode using an

Agilent 7000A Triple Quad equipped with an Agilent 7890A gas chromatograph and a J\&W Scientific DB-5MS+DG fused capillary column (60 m x $0.25 \mathrm{~mm}$ i.d., $0.25 \mu \mathrm{m}$ film 
thickness, $10 \mathrm{~m}$ guard column) using He as carrier gas. The GC oven was ramped from $40^{\circ} \mathrm{C}$ (2 min.) to $325^{\circ} \mathrm{C}$ at $4^{\circ} \mathrm{C} / \mathrm{min}$, holding for $25.75 \mathrm{~min}$. The carrier flow was ramped from $1.2 \mathrm{~mL} / \mathrm{min}$. (0 $\mathrm{min}$.) to $3.2 \mathrm{~mL} / \mathrm{min}$. Samples were injected in cold splitless mode at $45^{\circ} \mathrm{C}$ and the injector was ramped at $700^{\circ} \mathrm{C} / \mathrm{min}$ to $300^{\circ} \mathrm{C}$. The MS source was operated in EI-mode at $300^{\circ} \mathrm{C}$ with an ionization energy of $-70 \mathrm{eV}$. The number of molecular ion to fragment transitions varied throughout the run; dwell time was adjusted as needed to produce 3.5 cycles/second. MS1 \& MS2 resolution was set to "widest". The collision energy for $\beta$-carotane and $\gamma$-carotane was $5 \mathrm{eV}$, and it was $3 \mathrm{eV}$ for chlorobactane, okenane, paleorenieratane \& isorenieratane. Identification of all carotenoids was achieved by comparison of mass spectra and relative retention times to published data derived from full scan GC-MS.

A dilution series of synthetic standards were analyzed by GC-MRM-MS to estimate a limit of detection. An aliquot of $\beta$-carotene was catalytically hydrogenated to $28.6 \mathrm{mg}$ of $\beta$-carotane using $\mathrm{PtO}_{2}$, hexane, and acetic acid while continuously stirring and bubbling $\mathrm{H}_{2}$ (g). The purity of the hydrogenation product was tested by $\mathrm{GC}$, and after GC-purity was achieved, a set of $\beta$-carotane standards of known concentrations was prepared. The concentrations of the isorenieratane and chlorobactane synthetic standards (courtesy of Phillipe Schaeffer) were determined by comparison of the gas chromatography/ flame ionization detection (GC-FID) peak area to the GC-FID peak area of a $\beta$-carotane standard of known concentration. GC-MRM-MS analysis of a dilution series of $\beta$-carotane, isorenieratane, and chlorobactane standards indicates that saturated $\mathrm{C}_{40}$ carotanes, diaromatic $\mathrm{C}_{40}$ carotenoids, and monoaromatic $\mathrm{C}_{40}$ carotenoids can be detected to concentrations as low as $\sim 10 \mathrm{pg}$.

\subsubsection{Construction of the Stratigraphic Distribution Plot}

Sinninghe Damsté and Schouten (Sinninghe Damsté and Schouten, 2006) documented the occurrence of aromatic carotenoid derivatives in ancient depositional 
systems through geologic time. This list was updated and modified to only include isorenieratane, chlorobactane, paleorenieratane, and okenane in marine environments. The unsaturated precursors were included in addition to the hydrogenated diagenetic products so occurrences of aromatic $\mathrm{C}_{40}$ carotenoids in geologically young or modern marine samples were not neglected. Table S1 includes the references used to build figure 4A. During some intervals, particularly Mesozoic OAEs, there are many reports of carotenoid detection, but in order to avoid redundancy, table S1 only lists the most comprehensive references for a given time interval, emphasizing the oldest references where possible.

The geologic time scale was scaled vertically, but the vertical time scale was multiplied by a factor of 6.25 in the Precambrian compared to the Phanerozoic. A published report of a $\mathrm{C}_{40}$ carotenoid was treated equally as single band of constant thickness because age control was rarely good enough to determine the precise duration of carotenoid occurrence. A slightly thicker band was used to represent the new tandem mass spectrometry results in figure $4 \mathrm{~B}$. A dashed vertical line was used to denote age uncertainty for some of the oils analyzed for this study where the uncertainty was greater than the band thickness. Table 1 lists the sample information for the samples analyzed by tandem mass spectrometry for this study. The detection of isorenieratane, chlorobactane, paleorenieratane, and/or okenane in these samples was recorded in Table 1. Samples containing chlorobactane, okenane, and/or paleorenieratane always contained additional carotenoids, such as isorenieratane, renieratane, renierapurpurane, $\beta$-isorenieratane, $\beta$-renierapurpurane, $\beta$-carotane, and/or $\gamma$-carotane. However, a band representing the new results was placed into figure $4 \mathrm{~B}$ only if that specific carotenoid had not been reported in the literature during that time interval. 


\subsubsection{REFERENCES}

Armstrong HA, Abbott GD, Turner BR, Makhlouf IM, Muhammad AB, Pedentchouk N, Peters H (2009) Black shale deposition in an Upper Ordovician-Silurian permanently stratified, peri-glacial basin, southern Jordan. Palaeogeography, Palaeoclimatology, Palaeoecology, 273, 368-377.

Behrens A, Wilkes H, Schaeffer P, Clegg H, Albrecht P (1998) Molecular characterization of organic matter in sediments from the Keg River formation (Elk Point group), western Canada sedimentary basin. Organic Geochemistry, 29, 1905-1920.

Boreham CJ, Ambrose GJ (2005) Cambrian petroleum systems in the southern Georgina Basin, Northern Territory, Australia. In: Central Australian Basins Symposium petroleum and minerals potential, Alice Springs, NT., pp. 254-281.

Brocks JJ, Love GD, Summons RE, Knoll AH, Logan GA, Bowden SA (2005) Biomarker evidence for green and purple sulphur bacteria in a stratified Palaeoproterozoic sea. Nature, 437, 866870.

Cao C, Love GD, Hays L, Wang W, Shen S, Summons RE (2009) Biogeochemical evidence for euxinic oceans and ecological disturbance presaging the end-Permian mass extinction event. Earth and Planetary Science Letters, 281, 188-201.

French KL, Sepúlveda J, Trabucho-Alexandre J, Gröcke DR, Summons RE (2014) Organic geochemistry of the early Toarcian oceanic anoxic event in Hawsker Bottoms, Yorkshire, England. Earth and Planetary Science Letters, 390, 116-127.

Grice K, Cao C, Love G, Böttcher ME, Twitchett R, Grosjean E, Summons RE, Turgeon S, Dunning W, Jin Y (2005) Photic zone euxinia during the Permian-Triassic superanoxic event. Science, 307, 706-709.

Grice K, Schaeffer P, Schwark L, Maxwell JR (1996) Molecular indicators of palaeoenvironmental conditions in an immature Permian shale (Kupferschiefer, Lower Rhine Basin, north-west Germany) from free and S-bound lipids. Organic Geochemistry, 25, 131-147.

Hartgers WA, Sinninghe Damsté JS, Koopmans MP, de Leeuw JW (1993) Sedimentary evidence for a diaromatic carotenoid with an unprecedented aromatic substitution pattern. Journal of the Chemical Society Chemical Communications, 23, 1715-1716.

Hartgers WA, Sinninghe Damsté JS, Requejo AG, Allan J, Hayes JM, Ling Y, Xie TM, Primack J, de Leeuw JW (1994) A molecular and carbon isotopic study towards the origin and diagenetic fate of diaromatic carotenoids. Organic Geochemistry, 22, 703-725.

Hays L (2010) Biogeochemical Proxies for Environmental and Biotic Conditions at the Permian- 
Triassic Boundary: MIT dissertation, p. 3-287.

Heimhofer U, Hesselbo SP, Pancost RD, Martill DM, Hochuli PA, Guzzo JVP (2008) Evidence for photic-zone euxinia in the Early Albian Santana Formation (Araripe Basin, NE Brazil). Terra Nova, 20, 347-354.

Joachimski M, Ostertag-Henning C, Pancost R, Strauss H, Freeman K, Littke R, Sinninghe Damsté JS, Racki G (2001) Water column anoxia, enhanced productivity and concomitant changes in $\delta^{13} \mathrm{C}$ and $\delta^{34} \mathrm{~S}$ across the Frasnian-Famennian boundary (Kowala-Holy Cross Mountains/Poland). Chemical Geology, 175, 109-131.

Kohnen MEL, Schouten S, Sinninghe Damsté JS, de Leeuw JW, Merritt DA, Hayes JM (1992) Recognition of paleobiochemicals by a combined molecular sulfur and isotope geochemical approach. Science, 256, 358-362.

Koopmans MP, Köster J, Van Kaam-Peters HME, Kenig F, Schouten S, Hartgers WA, de Leeuw JW, Sinninghe Damsté JS (1996) Diagenetic and catagenetic products of isorenieratene: Molecular indicators for photic zone anoxia. Geochemica et Cosmochimica Acta, 60, 4467-4496.

Kuypers M, Pancost R, Nijenhuis I, Sinninghe Damsté JS (2002) Enhanced productivity led to increased organic carbon burial in the euxinic North Atlantic basin during the late Cenomanian oceanic anoxic event. Paleoceanography, 17, 1050.

Marynowski L, Filipiak P, Pisarzowska A (2008) Organic geochemistry and palynofacies of the Early-Middle Frasnian transition (Late Devonian) of the Holy Cross Mountains, Southern Poland. Palaeogeography, Palaeoclimatology, Palaeoecology, 269, 152-165.

Naeher S, Geraga M, Papatheodorou G, Ferentinos G, Kaberi H, Schubert CJ (2012) Environmental variations in a semi-enclosed embayment (Amvrakikos Gulf, Greece) reconstructions based on benthic foraminifera abundance and lipid biomarker pattern. Biogeosciences, 9, 5081-5094.

Pancost RD, Freeman KH, Patzkowsky ME, Wavrek DA, Collister JW (1998) Molecular indicators of redox and marine photoautotroph composition in the late Middle Ordovician of Iowa, U.S.A. Organic Geochemistry, 29, 1649-1662.

Passier HF, Bosch H.-J., Nijenhuis IA, Lourens LJ, Böttcher ME, Leenders A, Sinninghe Damsté JS, de Lange GJ, de Leeuw JW (1999) Sulphidic Mediterranean surface waters during Pliocene sapropel formation. Nature, 397, p. 146-149.

Racka M, Marynowski L, Filipiak P, Sobstel M, Pisarzowska A, Bond DPG (2010) Anoxic Annulata Events in the Late Famennian of the Holy Cross Mountains (Southern Poland): Geochemical and palaeontological record. Palaeogeography, Palaeoclimatology, Palaeoecology, 297, 549-575. 
Repeta DJ (1993) A high resolution historical record of Holocene anoxygenic primary production in the Black Sea. Geochemica et Cosmochimica Acta, 57, 4337-4342.

Repeta DJ, Simpson DJ, Jorgenson BB, Jannasch HW (1989) Evidence for anoxygenic photosynthesis from the distribution of bacteriochlorophylls in the Black Sea. Nature, 342, 69-72.

Requejo AG, Creaney S, Gray NR, Cole KS (1992) Aryl isoprenoids and diaromatic carotenoids in Paleozoic source rocks and oils from the Western Canada and Willston Basins. Organic Geochemistry, 19, 245-264.

Richoz S, van de Schootbrugge B, Pross J, Püttmann W, Quan TM, Lindström S, Heunisch C, Fiebig J, Maquil R, Schouten S, Hauzenberger CA, Wignall PB (2012) Hydrogen sulphide poisoning of shallow seas following the end-Triassic extinction. Nature Geoscience, 5, 662667.

Saito R, Oba M, Kaiho K, Schaeffer P, Adam P, Takahashi S, Nara FW, Chen Z.-Q., Tong J, Tsuchiya N (2014) Extreme euxinia prior to the Middle Triassic biotic recovery from the latest Permian mass extinction. Organic Geochemistry, 73, 113-122.

Schaeffer P, Reiss C, Albrecht P (1995) Geochemical study of macromolecular organic matter from sulfur-rich sediments of evaporitic origin (Messinian of Sicily) by chemical degradation. Organic Geochemistry, 23, 567-581.

Schouten S, van Kaam-Peters H, Rijpstra W, Schoell M, Sinninghe Damsté JS (2000) Effects of an oceanic anoxic event on the stable carbon isotopic composition of Early Toarcian carbon. American Journal of Science, 300, 1-22.

Schwab VF, Spangenberg JE (2007) Molecular and isotopic characterization of biomarkers in the Frick Swiss Jura sediments: A palaeoenvironmental reconstruction on the northern Tethys margin. Organic Geochemistry, 38, 419-439.

Schwark L, Püttmann W (1990) Aromatic hydrocarbon composition of Permian Kupferschiefer in the Lower Rhine Basin, NW Germany. Organic Geochemistry, 16, 749-761.

Sinninghe Damsté JS, Schouten S (2006) Biological markers for anoxia in the photic zone of the water column. In: Marine organic matter: Biomarkers, isotopes and DNA. The Handbook of Environmental Handbook. Springer Berlin Heidelberg, pp. 127-163.

Sluijs A, Schouten S, Donders TH, Schoon PL, Röhl U, Reichart G.-J., Sangiorgi F, Kim J.-H., Sinninghe Damsté JS, Brinkhuis H (2009) Warm and wet conditions in the Arctic region during Eocene Thermal Maximum 2. Nature Geoscience, 2, 777-780.

Sluijs A, Schouten S, Pagani M, Woltering M, Brinkhuis H, Sinninghe Damsté JS, Dickens GR, Huber M, Reichart G, Stein R, Matthiessen J, Lourens LJ, Pedentchouk N, Backman J, Moran 
K (2006) Subtropical Arctic Ocean temperatures during the Palaeocene/Eocene thermal maximum. Nature, 441, 610-613.

Smittenberg RH, Pancost RD, Hopmans EC, Paetzel M, Sinninghe Damsté JS (2004) A 400-year record of environmental change in an euxinic fjord as revealed by the sedimentary biomarker record. Palaeogeography, Palaeoclimatology, Palaeoecology, 202, 331-351.

van Bentum EC, Hetzel A, Brumsack H.-J., Forster A, Reichart G.-J., Sinninghe Damsté JS (2009) Reconstruction of water column anoxia in the equatorial Atlantic during the CenomanianTuronian oceanic anoxic event using biomarker and trace metal proxies. Palaeogeography, Palaeoclimatology, Palaeoecology, 280, 489-498.

van Breugel Y, Schouten S, Tsikos H, Erba E, Price GD, Sinninghe Damsté JS (2007) Synchronous negative carbon isotope shifts in marine and terrestrial biomarkers at the onset of the early Aptian oceanic anoxic event 1a: Evidence for the release of ${ }^{13} \mathrm{C}$-depleted carbon into the atmosphere. Paleoceanography, 22, PA1210.

Van Kaam-Peters HME, Sinninghe Damsté JS (1997) Characterization of an extremely organic sulphur-rich, 150 Ma old carbonaceous rock: palaeoenvironmental implications. Organic Geochemistry, 371-397.

Wagner T, Sinninghe Damsté JS, Hofmann P, Beckmann B (2004) Euxinia and primary production in Late Cretaceous eastern equatorial Atlantic surface waters fostered orbitally driven formation of marine black shales. Paleoceanography, 19, PA3009. 
Table S1. Literature References for Construction of Figure 4A

\begin{tabular}{|c|c|c|c|}
\hline Age & $\begin{array}{l}\text { Aromatic C40 } \\
\text { Carotenoid* }\end{array}$ & Locality & References \\
\hline Holocene & 1 & Black Sea & (Repeta et al., 1989; Repeta, 1993) \\
\hline Holocene & 1,2 & Amvrakikos Gulf (Greece) & (Naeher et al., 2012) \\
\hline Holocene & 1,4 & Kyllaren Fjord & (Smittenberg et al., 2004) \\
\hline Pliocene & 1 & Eastern Mediterranean Sapropels (ODP) & (Passier et al., 1999) \\
\hline Messinian & 1 & Vena del Gesso Basin (Italy) & (Kohnen et al., 1992) \\
\hline Messinian & 1,2 & Gebellina Marl (Sicily) & (Schaeffer et al., 1995) \\
\hline Oligocene & 1 & Menilite Formation (Poland) & (Koopmans et al., 1996) \\
\hline ETM-2 & 1 & Arctic Ocean (IODP) & (Sluijs et al., 2009) \\
\hline PETM & 1 & Arctic Ocean (IODP) & (Sluijs et al., 2006) \\
\hline OAE-3 & 1,2 & Deep Ivorian Basin (ODP) & (Wagner et al., 2004) \\
\hline Late Turonian & 1 & Canje Formation (British Guyana) & (Koopmans et al., 1996) \\
\hline OAE-2 & 1,2 & North Atlantic (DSDP \& ODP) & (Kuypers et al., 2002; van Bentum et al., 2009) \\
\hline OAE-1b & 1 & Santana Formation (Brazil) & (Heimhofer et al., 2008) \\
\hline OAE-1a & 1 & Venetian Alps (Italy) & (van Breugel et al., 2007) \\
\hline Late Jurassic & 1 & Kimmeridge Clay (UK) & (Koopmans et al., 1996) \\
\hline Late Jurassic & 1,2 & Calcaires en Plaquettes (France) & $\begin{array}{l}\text { (Koopmans et al., 1996; Van Kaam-Peters and } \\
\text { Sinninghe Damsté, 1997) }\end{array}$ \\
\hline Middle Callovian & 1 & Oxford Clay (UK) & (Koopmans et al., 1996) \\
\hline Toarcian & 1,2 & $\begin{array}{l}\text { Northern Europe (Allgäu; Schistes Cartons; } \\
\text { Posidionia) }\end{array}$ & (Koopmans et al., 1996; Schouten et al., 2000) \\
\hline Toarcian OAE & $1,2,4$ & $\begin{array}{l}\text { Hawsker Bottoms; Cleveland Basin UK; MRM } \\
\text { rock extract }\end{array}$ & (French et al., 2014) \\
\hline $\begin{array}{l}\text { Middle Sinemurian to Middle } \\
\text { Hettangian }\end{array}$ & 1 & Frick Swiss Jura & (Schwab and Spangenberg, 2007) \\
\hline
\end{tabular}




\begin{tabular}{|c|c|c|c|}
\hline Upper Hettangian & 1 & Northern Europe & (Richoz et al., 2012) \\
\hline Norian-Rhaetian & 1 & Kössen Marl (Hungary) & (Koopmans et al., 1996) \\
\hline Norian & 1 & Hauptdolomit (Germany) & (Koopmans et al., 1996) \\
\hline $\begin{array}{l}\text { Early-Mid Triassic; Olenekian-- } \\
\text { Anisian }\end{array}$ & 2,4 & Chaohu sections, South China & (Saito et al., 2014) \\
\hline Early Triassic & 1 & Perth Basin (Australia) & (Grice et al., 2005) \\
\hline Early Triassic & 1,2 & Peace River (Canada) & (Hays, 2010) \\
\hline Early Triassic to Late Permian & 1,2 & Meishan (China) & (Cao et al., 2009; Hays, 2010) \\
\hline Late Permian & 1 & Kupferschiefer (Germany) & $\begin{array}{l}\text { (Schwark and Püttmann, 1990; Grice et al., } \\
\text { 1996) }\end{array}$ \\
\hline Late Carboniferous & 1 & Minnelusa Formation (USA) & (Koopmans et al., 1996) \\
\hline Early Carboniferous & 1,3 & Exshaw Formation (Canada) & (Koopmans et al., 1996) \\
\hline Upper Famennian & 1,3 & Holy Cross Mountains (Poland) & (Racka et al., 2010) \\
\hline Frasnian/Famennian & 1,3 & Holy Cross Mountains (Poland) & (Joachimski et al., 2001) \\
\hline Frasnain & 1,3 & Duvernay Fm (Canada) & $\begin{array}{l}\text { (Requejo et al., 1992; Hartgers et al., 1993; } \\
\text { Hartgers et al., 1994) }\end{array}$ \\
\hline Early-Mid Frasnian transition & 1,3 & Holy Cross Mountains (Poland) & (Marynowski et al., 2008) \\
\hline Middle Devonian; Givetian & 1,3 & Keg River Formation (Canada) & (Behrens et al., 1998) \\
\hline Late Ordovician-Early Silurian & 1,3 & Batra Formation (Jordon) & (Armstrong et al., 2009) \\
\hline Late Ordovician & 1,3 & Boas Oil Shale (Canada) & (Koopmans et al., 1996) \\
\hline Caradocian & 1 & Decorah Formation (USA) & (Pancost et al., 1998) \\
\hline Middle Ordovician & 1,3 & Womble Shale (USA) & (Koopmans et al., 1996) \\
\hline Late Cambrian & 1 & Hagen Member (Australia) & (Boreham and Ambrose, 2005) \\
\hline Middle Cambrian & 1 & Arthur Creek Formation (Australia) & (Boreham and Ambrose, 2005) \\
\hline Middle Cambrian & 1 & Thorntonia Limestone (Australia) & (Boreham and Ambrose, 2005) \\
\hline Late Paleoproterozoic & $1,2,4$ & Barney Creek Formation (Australia) & (Brocks et al., 2005) \\
\hline
\end{tabular}

*Isorenieratane and Isorenieratene are designated by $\mathbf{1}$. Chlorobactane or Chlorobactene are designated by 2. Paleorenieratane is designated by $\mathbf{3}$. Okenane or Okenone are designated by 4 . 


\section{Chapter 5:}

Evaluating the Benguela upwelling system for anoxygenic photosynthesis as a modern analog of Oceanic Anoxic Events 


\subsection{INTRODUCTION}

The discovery of a sedimentary geologic record of degradation products of photosynthetic pigments belonging to green sulfur bacteria (1) has enabled the identification of past episodes of photic zone euxinia (PZE). PZE is the occurrence of sulfide in the sunlit layer of the water column and has been recorded in sedimentary rocks deposited more than 1.6 billion years ago (2). These sedimentary carotenoids have provided the basis for testing Proterozoic redox structure $(2,3)$, evaluating sulfide poisoning as a possible kill mechanism during mass extinctions and oceanic anoxic events (OAEs) (e.g. 4, 5), and characterizing the marine chemistry in contemporary ocean basins (6-8). However, the compilation of carotenoid markers of green and purple sulfur bacteria (GSB and PSB, respectively) through geologic time (detailed in chapter 4) raises the question of whether these biomarkers exclusively reflect an autochthonous water column signature, which has been the classic interpretation (e.g. 5, 6, 9-16).

While allochthonous input and benthic mat production of GSB and PSB carotenoids may be critical aspects of interpreting the new patterns of carotenoid distribution in geologic time (Chapter 4), this study will focus on the "no analog" problem discussed in chapters 3 and 4 . One of the driving questions is whether or not these paleoredox proxies truly face a "no analog" problem or have these compounds not been found in relevant analog systems because of inadequate investigation. When possible, the most appropriate contemporary analog systems should be used to inform our interpretation of past environmental conditions. As this problem currently stands, the use of sedimentary GSB and PSB carotenoids in ancient samples to estimate the depth of sulfide penetration into the photic zone is based on studies of select highly restricted environments, such as the Black Sea, high latitude fjords, and stratified lakes (e.g. 17, 18). Yet despite the restricted nature of all of these modern settings, basin restriction is rarely inferred from the presence of these compounds in geologic samples. Instead, the 
phototrophic sulfur bacterial carotenoids, in conjunction with inorganic geochemical evidence, have been used to build a model of expanded sulfidic oxygen minimum zones (OMZs) during Phanerozoic OAEs. This is in contrast to the more traditional "ocean stagnation" model which has been shown to be physically unsustainable (19-24). However, do observations of transiently sulfidic OMZs in the modern ocean provide evidence for GSB and PSB production, thereby strengthening the traditional paleoredox interpretation of the $\mathrm{C}_{40}$ carotenoids?

Although there is no observational evidence for this phenomena so far, model results show that a sulfidic water column can develop when upwelling rates and phosphate supply are sufficient, thereby accommodating GSB production and isorenieratane observations in ancient sedimentary rocks $(18,24)$. However, there are no models or environmental studies, to date, that verify whether reduced sulfur and light requirements for PSB and okenone production can be established and sustained in unrestricted marine water columns under Phanerozoic atmospheric oxygen concentrations. Even if models were able to demonstrate that a stable sulfidic chemocline could develop in unrestricted marine water columns at shallow enough depths for PSB and okenone production $(<24 \mathrm{~m})(17)$, the water column irradiance would also have to be assessed in parallel with the development of sulfidic conditions. As seen in satellite images of the Namibian margin, the water column becomes turbid when elemental sulfur is generated during sulfide eruptions, which likely affects light penetration through the water column $(25,26)$. Interestingly, gene sequences that cluster with green sulfur bacteria have been reported from the Peruvian oxygen minimum zone (OMZ) and the Benguela upwelling system off the coast of Namibia (27, 28), yet there is no indendent geochemical evidence that supports green sulfur bacterial activity in these systems. Coupled with the possibility of a "cryptic sulfur cycle" (29, 30 ), these observations and gaps in our understanding underscore the need to evaluate 
transiently sulfidic OMZs for observational evidence of green and purple sulfur bacteria and their pigments.

Coastal upwelling of nutrient rich waters along subtropical eastern boundaries, combined with poorly ventilated subsurface waters of the gyre "shadow zones", gives rise to the unique geochemistry and ecosystems of OMZs. These highly productive regions are, therefore, in close association with subsurface waters that have some of the lowest oxygen concentrations in the global ocean (31). While studies of nitrogen cycle dynamics in OMZs have been a key focus of oceanographic research (e.g. 32-34), it has been proposed that a sulfur cycle operates in the OMZs as well $(27,29,30)$. Sulfide plumes have been detected in multiple OMZs $(27,35)$, but the frequent and intense sulfide eruptions of the Benguela upwelling system (25-27, 36-38), located along the northeastern part of the South Atlantic subtropical gyre, makes it a prime natural laboratory to test whether phototrophic sulfide oxidation by GSB and/or PSB is an important sulfide removal mechanism in the water column, as is the case for chemolithotrophic oxidation of sulfide by $\gamma$ - and $\varepsilon$-proteobacteria (27). In this study, marine sediments from the Namibian shelf were analyzed for photosynthetic pigments of GSB and PSB, including carotenoids and bacteriochlorophylls, with the expectation that the sediments will have integrated the water column signature over the course of many sulfide eruptions. If present, the detection of GSB and PSB pigments would add an important independent geochemical line of evidence for a "cryptic sulfur cycle", would provide an avenue to reconstruct a historical record of sulfide eruptions on the Namibian margin, and would strengthen the environmental interpretation of GSB and PSB fossil pigments in the geologic record.

\subsection{METHODS}

Sample Collection and Curation 
Marine sediment samples were collected in March 2014 during a cruise of the RV Mirabilis belonging to the Namibian Ministry of Fisheries and Marine Resources. Samples were collected at regularly monitored stations on transects of the $23^{\circ} \mathrm{S}, 24^{\circ} \mathrm{S}$, $25^{\circ} \mathrm{S}, 26^{\circ} \mathrm{S}, 27^{\circ} \mathrm{S}$ latitudes in water depths ranging from $30 \mathrm{~m}$ to $260 \mathrm{~m}$ (Fig. 1). A combination of sediment cores and core tops were collected, but grab samples were collected when coring was not possible. Many of the recovered samples, particularly between $23-25^{\circ} \mathrm{S}$, smelled strongly of sulfide. If the whole core was collected for pigment analyses, it was sectioned on deck after recovery into 1 or 2 inch thick core samples. The samples were placed into labeled plastic bags and frozen in a dark freezer room at $-20^{\circ} \mathrm{C}$. Because the samples were collected specifically for pigment work they were kept frozen and dark in a cooler after collection and during transport to MIT. They remained frozen at $-20^{\circ} \mathrm{C}$ in a dark freezer room as wet sediment until pigment extraction.

\section{Pigment extraction}

The samples were prepared and extracted in the dark or under low light conditions. The sediments were freeze-dried for approximately 1 day until the water was removed. The freeze-dried sediment was weighed into a clean centrifuge tube. A range of 1.5 to $11.3 \mathrm{~g}$ of dried sediment in clean glass centrifuge tubes was extracted by sonication with acetone in an ice bath that was covered to keep the samples dark (Table 1). The samples were sonicated in intervals of 15 minutes after which they were centrifuged, and the decanted acetone was collected as the pigment extract. This process was repeated seven times for each extract. The samples were so organic rich (39) that complete extraction was not possible using this extraction method. The combined acetone extracts were concentrated at room temperature under a gentle stream of $\mathrm{N}_{2}$. The samples were passed through a filter to remove particulates, and the extract was weighed. A 4-6 mg aliquot of the extract was transferred to an amber combusted glass insert and was 
brought up in a total of $200 \mu \mathrm{L}$ of acetone. During sample preparation, the sample and extract was handled in low light using amber glassware when possible or clear glassware that was covered with aluminum foil. A set of reference materials, which included a Fayetteville Green Lake (FGL; New York) sediment (40), a piece of salt pond mat (courtesy of D. Repeta) (41), spinach leaves, Chlorobium tepidum biomass (courtesy of D. Bryant) (42-44), was also prepared according to the same acetone extraction protocol. However, only the FGL sediments were freeze-dried prior to extraction, while the other reference material was extracted wet. An aliquot of the bacteriochlorophyll $a$ (Bchl a) standard from Rhodopseudomonas sphaeroides (Frontier Scientific) was demetallated using $98 \%$ formic acid in order to prepare a set of bacteriopheophytin $a$ standards.

\section{HPLC-MS analytical conditions}

The HPLC-MS method was modified after a combination of previous studies of pigments $(41,45,46)$ and based on preliminary analyses of reference materials, which included isolated isorenieratene (courtesy of D. Repeta), a salt pond mat extract, a FGL sediment extract, a Chlorobium tepidum biomass extract, a spinach extract, a $\beta$-carotene standard, a bacteriochlorophyll $a$ standard, and a bacteriopheophytin $a$ standard. The pigment analyses were performed on an Agilent 1200 series HPLC coupled to an Agilent 6520 Q-TOF mass spectrometer (MS), which was operated in auto MS/MS mode where a maximum of three precursors were selected for MS/MS fragmentation per cycle. An Agilent poroshell 120 EC-C 18 column $(2.1 \times 150 \mathrm{~mm}, 2.7 \mu \mathrm{m})$ column with a pre-column (EC-C $182.1 \times 5 \mathrm{~mm} ; 2.7 \mu \mathrm{m}$ ) was used to separate compounds according to the following solvent gradient: $20 \%$ solvent B ( 0 min) to $100 \%$ solvent B ( $28.5 \mathrm{~min}$.), hold $100 \%$ solvent B ( $48.5 \mathrm{~min}$ ), return to $20 \% \mathrm{~B}$ (50 min.), finally reconditioning at $20 \% \mathrm{~B}$ until a final time of 60 minutes. Solvent A was 80:20 (v:v) methanol:0.05 M ammonium acetate, and solvent B was 80:10:10 (v:v:v) methanol:acetonitrile:ethyl acetate. The 
solvents were HPLC grade purity. The solvent flow rate was set to $0.4 \mathrm{~mL} / \mathrm{min}$ and the column temperature was set to $35^{\circ} \mathrm{C}$. The APCI-MS was operated in positive ion mode, and the nebulizer was set to $40 \mathrm{psig}$. The APCI gas and vaporizer temperatures were set to $300^{\circ} \mathrm{C}$ and $400^{\circ} \mathrm{C}$. The drying gas flow rate was $6 \mathrm{~L} / \mathrm{min}$. The corona current was set to $5 \mu \mathrm{A}$, and the capillary voltage was $2000 \mathrm{~V}$.

Three dilution series of the $\beta$-carotene standard, the bacteriochlorophyll $a$ standard, and the bacteriopheophytin a standard were analyzed in the same sequence with the samples in order to evaluate the response of the instrument and method at different compound concentrations. The concentrations of each standard dilution series ranged from $25 \mathrm{ng} / \mu \mathrm{L}$ to $0.1 \mathrm{ng} / \mu \mathrm{L}(100 \mathrm{pg} / \mu \mathrm{L})$. An aliquot of $5 \mu \mathrm{L}$ was injected on column, so the on column masses of the dilution series ranged from $125 \mathrm{ng}$ to $0.5 \mathrm{ng}$ (500 pg). Although absolute detection limits were not determined, the lowest concentration of $100 \mathrm{pg} / \mu \mathrm{L}$ (or $500 \mathrm{pg}$ on column) was easily detectable for each of the standards. The stronger response observed for the bacteriopheophytin a compared to the bacteriochlorophyll $a$ (see Fig. 2) is in agreement with previous observations that have shown that the ionization efficiency is higher for demetalled chlorophylls (pheophytins) than chlorophylls (47). The $\beta$-carotene response was linear over the concentration range used for the dilution series $\left(\mathrm{R}^{2}=0.99\right)$. The bacteriopheophytin a response was more linear $\left(R^{2}=0.99\right)$ than the bacteriochlorophyll $a$ response $\left(R^{2}=0.94\right)$.

\section{Results and Discussion}

The compounds that were identified in the reference materials are listed in Table 2. The MS/MS method on the QTOF, as described above, provided diagnostic mass spectra that allowed the identification of compounds that had not been previously identified in the FGL sediment extracts. In agreement with previous analyses of FGL sediments and water column (40), okenone was the dominant peak in the base peak chromatogram 
(BPC) of the FGL sediment extract (Fig. 3). Isorenieratene and chlorobactene were not prominent peaks in the BPC, but they became apparent in the 529.4 and 533.4 extracted ion chromatograms (EIC; Fig. 4 and 5). The identifications of isorenieratene and chlorobactene were further confirmed by comparison of the retention time and mass spectra with those belonging to the isolated isorenieratene standard and the Chlorobium tepidum biomass extract (44), respectively (Fig. 3 and 4).

This finding suggests that lipid biomarkers in the sediments reflect the lipid and DNA profiles of the water column and microbial mat better than has been previously suggested for FGL (40). In other words, the carotenoid biomarker record in FGL accurately reflects the anoxygenic phototrophic community in the meromictic FGL. The most likely explanation for the difference in carotenoid detection between this study and earlier studies (40) is analytical sensitivity. The Meyer et al. (2011) pigment analysis method had on column detection limits of 2.2-2.5 $\mu \mathrm{g}$ for okenone, chlorobactene, and isorenieratene. In contrast, the limit of detection, although not conclusively determined for each of the specific carotenoids in this study, was approximated by the lowest member of the dilution series for $\beta$-carotene, which illustrates that the HPLC-QTOF-MS method easily detects an on column $\beta$-carotene mass of $500 \mathrm{pg}$. Because isorenieratene and chlorobactene are structurally similar to $\beta$-carotene, it is reasonable to expect that the HPLC-QTOF-MS method can also detect on column injected masses of $500 \mathrm{pg}$ or less. This is significantly less than the method described by Meyer et al. (2011). Alternatively, the sediment lipid profile may have lateral heterogeneities and depend on sample locality in the lake basin. Regardless, the detection of isorenieratene and chlorobactene in the FGL sediment extract confirms that the HPLC-QTOF-MS method is robust and can measure trace concentrations of carotenoids in complex sediment extracts. 
The retention times and mass spectra from the reference samples were used to screen the Namibian margin sediment extracts for trace concentrations of green and purple sulfur bacterial carotenoids (Table 2). Despite the targeted search, okenone, chlorobactene, and isorenieratene were not detected in any of the sediment extracts from the Namibian margin. However, the samples were not devoid of carotenoids. $\beta$ carotene was a dominant pigment in all of the Namibian sediment extracts (Table 2; Fig 6). This is not surprising since all algae, with the exception of cryptophytes and rhodophytes, produce $\beta$-carotene (48). So while $\beta$-carotene offers little taxonomic specificity, its detection demonstrates that the preservational conditions at the sample localities are conducive for carotenoid preservation.

However, bacteriochlorophylls and their degradation products can also be used as markers for green and purple sulfur bacteria. In addition to carotenoids, bacteriochlorophylls $a$ and $b$ and bacteriochlorophylls $a, c, d$, and $e$ are essential antenna or reaction center pigments in the photosynthetic apparatus of purple and green sulfur bacteria, respectively (49). Bacteriochlorophyll porphyrin rings can have multiple combinations of substitutions and are esterified to farnesol or other long chain alcohols (49). In environmental systems, the structural diversity is further enhanced with the addition of degradation products. Bacteriochlorophylls and their degradation products have been used as markers for green sulfur bacteria $(7,50-53)$.

Chlorophylls $a$ and $b$ and bacteriochlorophylls $a, c$, and $e$ were identified in the reference samples in conjunction with their respective degradation products (Table 2; Fig. 7). These identifications are based on comparison with standards, published occurrences, or inferred from mass spectra. The retention times and mass spectra for the identified compounds in the reference materials were used to systematically evaluate whether the Namibian sediment extracts also contained any of these compounds. No 
bacteriochlorophylls or bacteriopheophytins were identified in the Namibian margin sediment extracts (Table 2). Instead, the sediment extracts from the Namibian margin contained chlorophyll $a$, chlorophyll $a$ degradation products, and $\beta$-carotene as the dominant pigments (Fig. 6).

While the targeted search for green and purple sulfur bacterial pigments in the sediments from the Namibian margin does not provide support for the presence of GSB and PSB in this dynamic system, the results, of course, do not preclude the occurrence of GSB and PSB in the transiently sulfidic water column of the Benguela upwelling system. It is possible that analyses of additional samples from other stations that were not sampled, particularly to the north of Walvis Bay, could yield GSB and PSB pigments in future studies. Alternatively, exploring sediments from a broader depth distribution ( $<30 \mathrm{~m}$ and $>150 \mathrm{~m}$ ) may yet reveal PSB and GSB pigments in future studies. Finally, a significant component of carotenoids can be sulfur-bound in marine sediments and rocks, hinting that sulfurization could be a significant step in the diagenesis and eventual preservation of these compounds (54-59). Consequently, desulphurization could reveal sulfur-bound GSB and/or PSB carotenoids in future studies.

Nevertheless, the results described here further underscore that while gene sequences that cluster with Chlorobi have been identified in the Peruvian and Namibian OMZs (27, 28), there is still no tangible geochemical evidence that phototrophic sulfur bacteria are actively oxidizing sulfide as part of the "cryptic sulfur cycle" (30). Further lines of geochemical evidence must be examined in future work to substantiate the significance and capacity of the cryptic sulfur cycle in the Namibian shelf waters and in the global OMZs (29). Furthermore, the lack of a modern analog for green and purple sulfur bacteria in unrestricted, transiently sulfidic marine environments should be weighed 
more heavily in future paleoredox interpretations based on GSB and PSB pigment biomarkers.

\subsection{Conclusion}

The inability to detect GSB and PSB pigments in Namibian margin sediments underscores the "no analog" problem. The transiently sulfidic Benguela upwelling system exhibits some of the key characteristics that most directly and accurately translate to the preferred model for Phanerozoic OAEs, but all of the available evidence suggests that green and purple sulfur bacteria are not significant members of this modern analog system. Perhaps, the sulfidic chemocline that develops on the Namibian margin is too unstable and transient for the GSB and PSB to establish a community that is detectable using lipidomic approaches. Alternatively, the elemental sulfur that clouds the water column during sulfide eruptions may inhibit enough light from reaching the sulfidic zone of the water column, thereby preventing both physiological requirements from being simultaneously met. In light of the persisting lack of examples of GSB and PSB in modern unrestricted, transiently sulfidic marine environments, these results further highlight the need to better understand the transport pathways for allochthonous GSB and PSB pigments, microbial mat production of the PSB and GSB carotenoids, the biosynthetic pathways for these carotenoids, and the complete physiological diversity leading to the production of these carotenoids.

\subsection{Acknowledgements}

We thank the RGNO research course program in microbial and geochemical oceanography in upwelling ecosystems for funding and logistical support that made sample collection possible, Dan Repeta for supplying reference material and valuable insight, Xiaolei Liu and Emily Matys for assistance with the HPLC-MS instrumentation, 
and Don Bryant for supplying Chlorobium tepidum biomass. We also acknowledge funding from NSF and NASA Astrobiology Institute.

\subsection{References}

1. Summons RE, Powell T (1986) Chlorobiaceae in Palaeozoic seas revealed by biological markers, isotopes and geology. Nature 319:763-765.

2. Brocks JJ et al. (2005) Biomarker evidence for green and purple sulphur bacteria in a stratified Palaeoproterozoic sea. Nature 437:866-870.

3. Lyons TW, Reinhard CT, Scott C (2009) Redox Redux. Geobiol 7:489-494.

4. Kump LR, Pavlov A, Arthur MA (2005) Massive release of hydrogen sulfide to the surface ocean and atmosphere during intervals of oceanic anoxia. Geology 33:397-400.

5. Grice K et al. (2005) Photic zone euxinia during the Permian-Triassic superanoxic event. Science 307:706-709.

6. Passier HF et al. (1999) Sulphidic Mediterranean surface waters during Pliocene sapropel formation. Nature 397:146-149.

7. Repeta DJ, Simpson DJ, Jorgenson BB, Jannasch HW (1989) Evidence for anoxygenic photosynthesis from the distribution of bacteriochlorophylls in the Black Sea. Nature 342:69-72.

8. Repeta DJ (1993) A high resolution historical record of Holocene anoxygenic primary production in the Black Sea. Geochim Cosmochim Acta 57:4337-4342.

9. Sluijs A et al. (2006) Subtropical Arctic Ocean temperatures during the Palaeocene/Eocene thermal maximum. Nature 441:610-613.

10. Sluijs A et al. (2009) Warm and wet conditions in the Arctic region during Eocene Thermal Maximum 2. Nat Geosci 2:777-780.

11. Kuypers MMM et al. (2004) Orbital forcing of organic carbon burial in the proto-North Atlantic during oceanic anoxic event 2. Earth Planet Sci Lett 228:465-482.

12. Kuypers M, Pancost R, Nijenhuis I, Sinninghe Damsté JS (2002) Enhanced productivity led to increased organic carbon burial in the euxinic North Atlantic basin during the late Cenomanian oceanic anoxic event. Paleoceanography 17:1051. 
13. Cao C et al. (2009) Biogeochemical evidence for euxinic oceans and ecological disturbance presaging the end-Permian mass extinction event. Earth Planet Sci Lett 281:188-201.

14. Hays LE, Beatty TW, Henderson CM, Love GD, Summons RE (2007) Evidence for photic zone euxinia through the end-Permian mass extinction in the Panthalassic Ocean (Peace River Basin, Western Canada). Palaeoworld 16:39-50.

15. Marynowski L et al. (2011) Molecular and petrographic indicators of redox conditions and bacterial communities after the F/F mass extinction (Kowala, Holy Cross Mountains, Poland). Palaeogeogr Palaeoclimatol Palaeoecol 306:1-14.

16. Saito R et al. (2014) Extreme euxinia just prior to the Middle Triassic biotic recovery from the latest Permian mass extinction. Org Geochem 73:113-122.

17. Brocks JJ, Schaeffer P (2008) Okenane, a biomarker for purple sulfur bacteria (Chromatiaceae), and other new carotenoid derivatives from the 1640 Ma Barney Creek Formation. Geochim Cosmochim Acta 72:1396-1414.

18. Meyer K, Kump L (2008) Oceanic euxinia in Earth history: Causes and consequences. Annu Rev Earth Planet Sci 36:251-288.

19. Brennecka GA, Herrmann A, Algeo TJ, Anbar AD (2011) Rapid expansion of oceanic anoxia immediately before the end-Permian mass extinction. Proc Natl Acad Sci USA 108:17631-17634.

20. Owens JD et al. (2013) Sulfur isotopes track the global extent and dynamics of euxinia during Cretaceous Oceanic Anoxic Event 2. Proc Natl Acad Sci USA 110:18407-18412.

21. Winguth AME, Maier-Reimer E (2005) Causes of the marine productivity and oxygen changes associated with the Permian-Triassic boundary: A reevaluation with ocean general circulation models. Mar Geol 217:283-304.

22. Erbacher J, Huber BT, Norris RD, Markey M (2001) Increased thermohaline stratification as a possible cause for an ocean anoxic event in the Cretaceous period. Nature 409:325327.

23. Wilson PA, Norris RD (2001) Warm tropical ocean surface and global anoxia during the mid-Cretaceous period. Nature 412:425-429.

24. Meyer K, Kump L, Ridgwell A (2008) Biogeochemical controls on photic-zone euxinia during the end-Permian mass extinction. Geology 36:747-750.

25. Weeks SJ, Currie B, Bakun A (2002) Satellite imaging: Massive emissions of toxic gas in the Atlantic. Nature 415:493-494.

26. Weeks SJ, Currie B, Bakun A, Peard KR (2004) Hydrogen sulphide eruptions in the 
Atlantic Ocean off southern Africa: implications of a new view based on SeaWiFS satellite imagery. Deep-Sea Res Pt I 51:153-172.

27. Lavik G et al. (2009) Detoxification of sulphidic African shelf waters by blooming chemolithotrophs. Nature 457:581-585.

28. Stewart FJ, Ulloa O, DeLong EF (2012) Microbial metatranscriptomics in a permanent marine oxygen minimum zone. Environ Microbiol 14:23-40.

29. Johnston DT et al. (2014) Placing an upper limit on cryptic marine sulphur cycling. Nature 513:530-533.

30. Canfield DE et al. (2010) A Cryptic Sulfur Cycle in Oxygen-Minimum-Zone Waters off the Chilean Coast. Science 330:1375-1378.

31. Sarmiento J, Gruber N (2013) Ocean biogeochemical dynamics (Princeton University Press).

32. Deutsch C, Sarmiento J, Sigman D, Gruber N, Dunne JP (2007) Spatial coupling of nitrogen inputs and losses in the ocean. Nature 445:163-167.

33. Kuypers $\mathrm{M}$ et al. (2005) Massive nitrogen loss from the Benguela upwelling system through anaerobic ammonium oxidation. Proc Natl Acad Sci USA 102.

34. Gruber N, Sarmiento J (1997) Global patterns of marine nitrogen fixation and denitrification. Global Biogeochem Cy 11:235-266.

35. Schunck H et al. (2013) Giant Hydrogen Sulfide Plume in the Oxygen Minimum Zone off Peru Supports Chemolithoautotrophy. PLoS One 8:e68661.

36. Emeis KC et al. (2004) Shallow gas in shelf sediments of the Namibian coastal upwelling ecosystem. Cont Shelf Res 24:627-642.

37. Brüchert V, Currie B, Peard KR (2009) Hydrogen sulphide and methane emissions on the central Namibian shelf. Prog Oceanogr 83:169-179.

38. Brüchert V et al. (2003) Regulation of bacterial sulfate reduction and hydrogen sulfide fluxes in the central namibian coastal upwelling zone. Geochim Cosmochim Acta 67:45054518.

39. Inthorn M, Wagner T, Scheeder G, Zabel M (2006) Lateral transport controls distribution, quality, and burial of organic matter along continental slopes in high-productivity areas. Geology 34:205.

40. Meyer KM et al. (2011) Carotenoid biomarkers as an imperfect reflection of the anoxygenic phototrophic community in meromictic Fayetteville Green Lake. Geobiol 9:321-329. 
41. Kurian S et al. (2012) Seasonal occurrence of anoxygenic photosynthesis in Tillari and Selaulim reservoirs, Western India. Biogeosciences 9:2485-2495.

42. Maresca JA, Romberger SP, Bryant DA (2008) Isorenieratene Biosynthesis in Green Sulfur Bacteria Requires the Cooperative Actions of Two Carotenoid Cyclases. J Bacteriol 190:6384-6391.

43. Maresca JA, Graham JE, Bryant DA (2008) The biochemical basis for structural diversity in the carotenoids of chlorophototrophic bacteria. Photosynthesis Research 97:121-140.

44. Maresca JA, Bryant DA (2006) Two Genes Encoding New Carotenoid-Modifying Enzymes in the Green Sulfur Bacterium Chlorobium tepidum. J Bacteriol 188:6217-6223.

45. Airs RL, Atkinson JE, Keely BJ (2001) Development and application of a high resolution liquid chromatographic method for the analysis of complex pigment distributions. $J$ Chromatogr A 917:167-177.

46. Bovee R (2013) Lipidomic and Genomic Investigation of Mahoney Lake, B.C. Harvard University PhD Thesis, pp. 1-144.

47. Airs RL, Keely BJ (2000) A novel approach for sensitivity enhancement in atmospheric pressure chemical ionisation liquid chromatography/mass spectrometry of chlorophylls. ... Communications in Mass Spectrometry.

48. Jeffrey SW, Mantoura R, Wright SW (2005) Phytoplankton pigments in oceanography: guidelines to modern methods.

49. Scheer H (2006) An Overview of Chlorophylls and Bacteriochlorophylls: Biochemistry, Biophysics, Functions and Applications. In Chlorophylls and Bacteriochlorophylls: Biochemistry, Biophysics, Functions and Applications, eds Grimm B, Porra RJ, Rüdiger W, Scheer H (Springer Netherlands), pp 1-26.

50. Grice K, Schaeffer P, Schwark L, Maxwell JR (1996) Molecular indicators of palaeoenvironmental conditions in an immature Permian shale (Kupferschiefer, Lower Rhine Basin, north-west Germany) from free and S-bound lipids. Org Geochem 25:131-147.

51. Grice K, Schaeffer P, Schwark L, Maxwell JR (1997) Changes in palaeoenvironmental conditions during deposition of the Permian Kupferschiefer (Lower Rhine Basin, northwest Germany) inferred from molecular and isotopic compositions of biomarker components. Org Geochem 26:677-690.

52. Pancost R et al. (2004) Further evidence for the development of photic-zone euxinic conditions during Mesozoic oceanic anoxic events. J Geol Soc London 161:353-364.

53. Keely BJ (2006) Geochemistry of Chlorophylls in Chlorophylls and Bacteriochlorophylls 
Biochemistry, Biophysics, Functions and Applications, eds Grimm B, Porra RJ, Rüdiger W, Scheer H, pp 535-561.

54. Sinninghe Damsté JS, Schouten S (2006) Biological Markers for Anoxia in the Photic Zone of the Water Column. In The Handbook of Environmental Chemistry ed. Volkman JK (Springer-Verlag, Berlin/Heidelberg), pp. 127-163.

55. Sinninghe Damsté JS, Wakeham SG, Kohnen ME, Hayes JM, de Leeuw JW (1993) A 6,000year sedimentary molecular record of chemocline excursions in the Black Sea. Nature 362:827-829.

56. Sinninghe Damsté JS, Köster J (1998) A euxinic southern North Atlantic Ocean during the Cenomanian/Turonian oceanic anoxic event. Earth Planet Sci Lett 158:165-173.

57. Hartgers WA et al. (1994) A molecular and carbon isotopic study towards the origin and diagenetic fate of diaromatic carotenoids. Org Geochem 22:703-725.

58. Koopmans MP et al. (1996) Diagenetic and catagenetic products of isorenieratene: Molecular indicators for photic zone anoxia. Geochim Cosmochim Acta 60:4467-4496.

59. Kohnen MEL et al. (1992) Recognition of paleobiochemicals by a combined molecular sulfur and isotope geochemical approach. Science 256:358-362. 


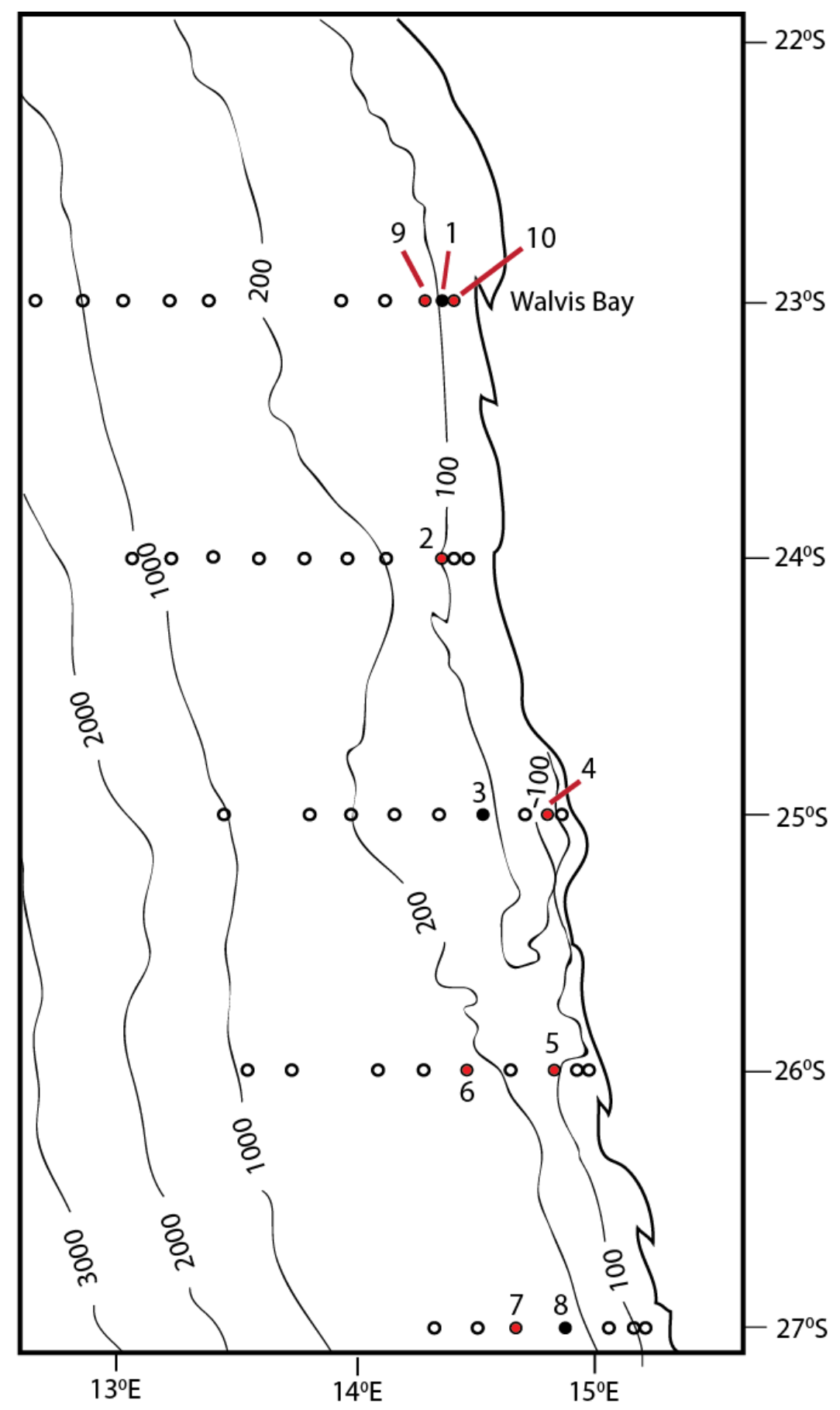

Figure 1. Map of sample stations on Namibian shelf modified from Lavik et al., 2009. Regularly monitored stations are marked with circles. The stations that were visited during the March 2014 cruise are marked by solid circles, where the red filled circle represent the stations where samples were collected and the black filled stations represent stations where samples were not collected. The stations are numbered in the order in which they were sampled. The full station names are provided in Table 1 according to the number labels here. 

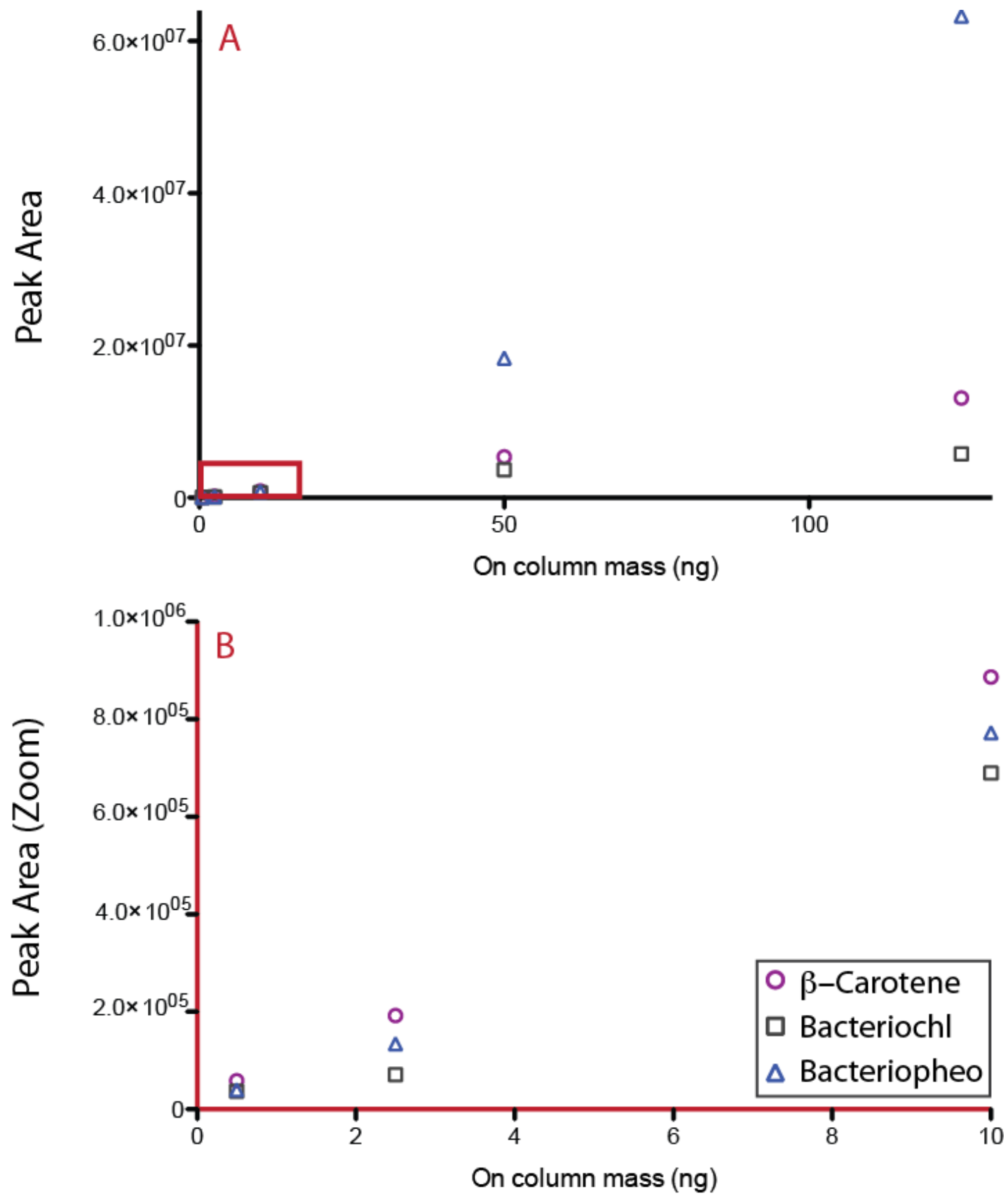

Figure 2. Response of HPLC-QTOF-MS for $\beta$-carotene, bacteriochlorophyll, bacteriopheophytin where injected standard mass (ng) is plotted against peak area (counts*sec). Panel B is a zoom on the area marked by the red box in panel A. 

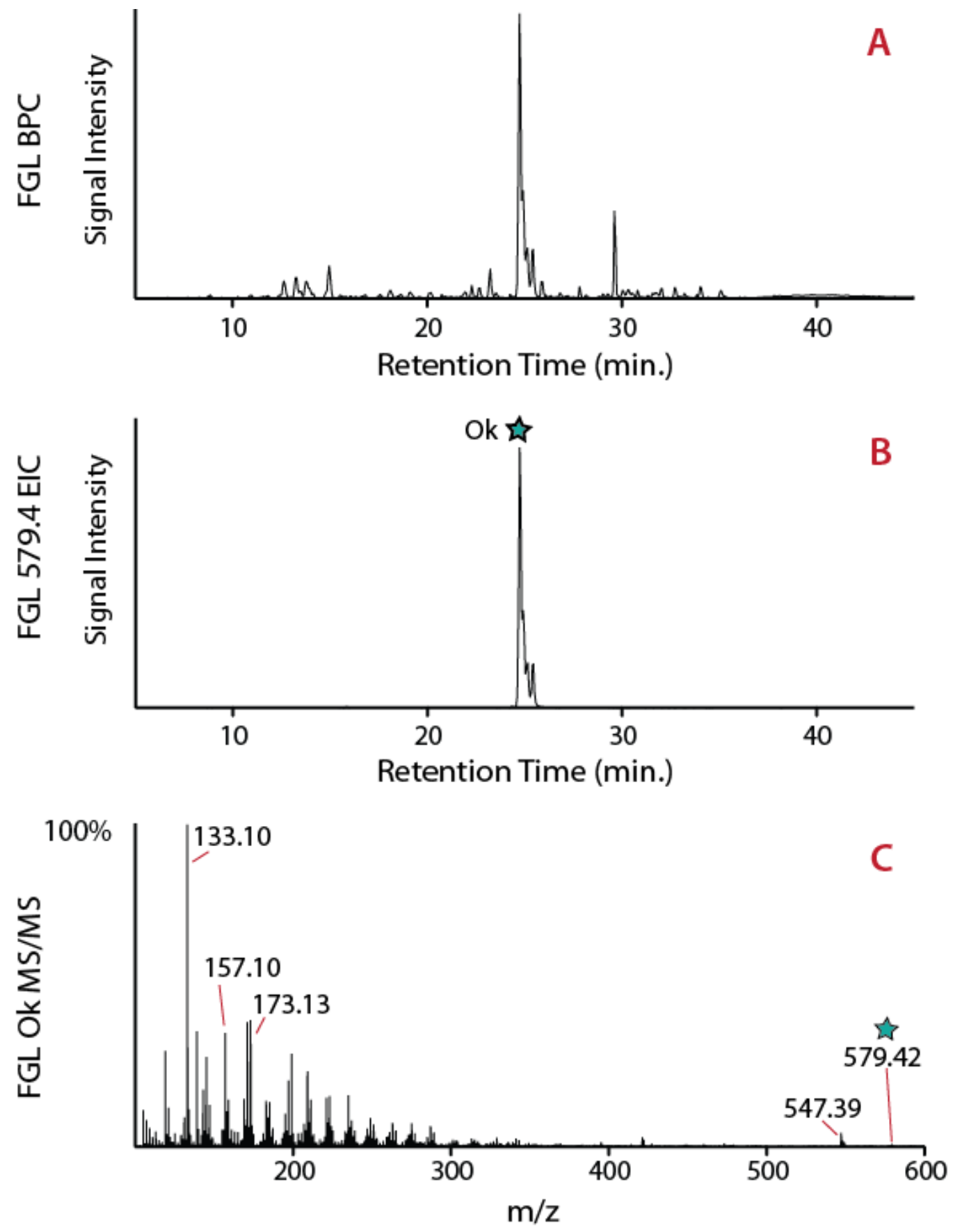

Figure 3. FGL sediment extract as an okenone reference. A) The base peak chromatogram (BPC) shows that the largest peak in the FGL extract is okenone (Ok). B) The extracted ion chromatogram (EIC) for 579.4 only shows okenone. C) A MS/MS spectrum taken from the starred retention time in $\mathrm{B}$ for the molecular mass that is starred in C confirms that this compound is okenone, which is further confirmed by FGL peak identification in Meyers et al. 2011. 

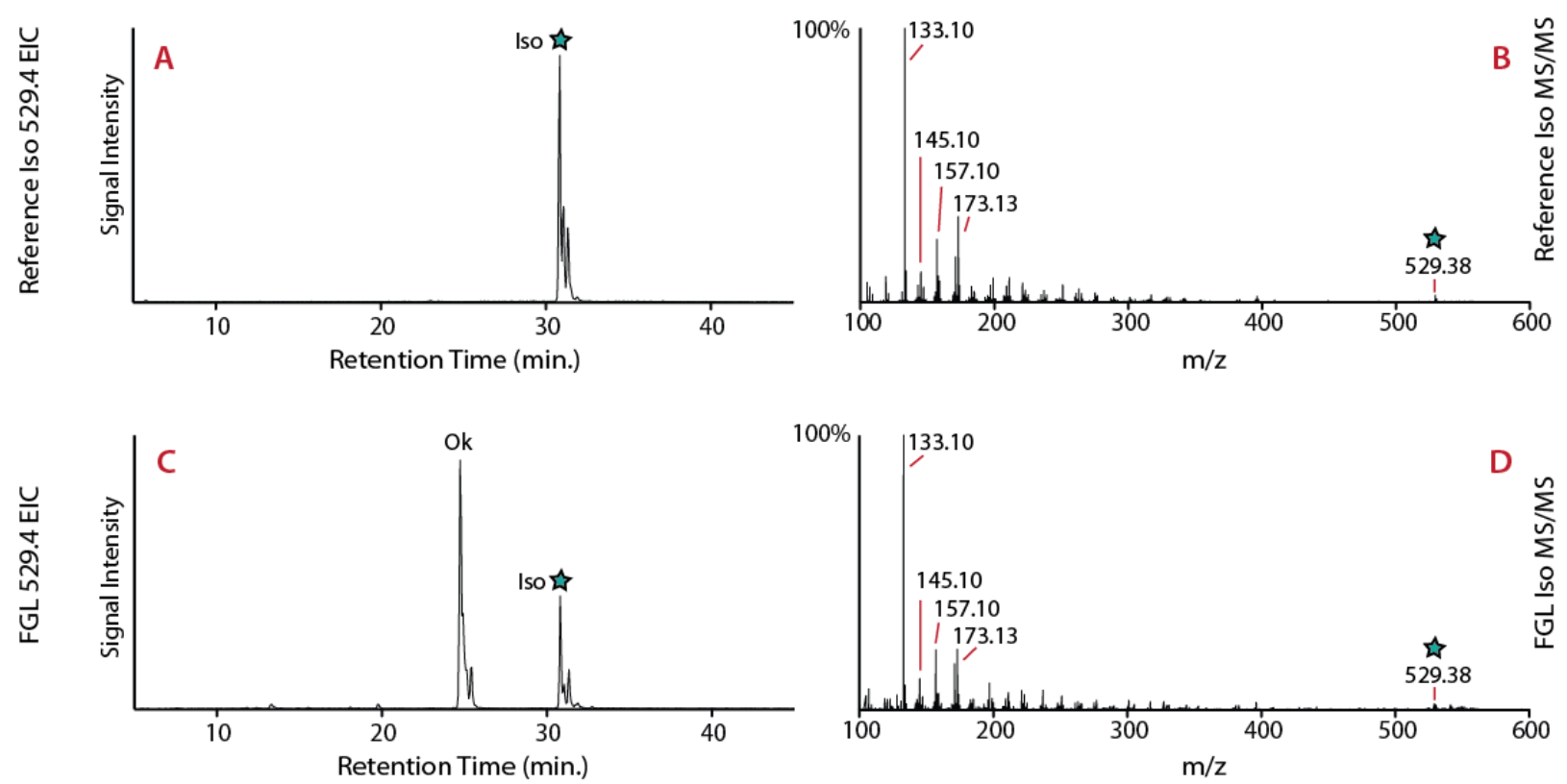

Figure 4. Identification of isorenieratene in FGL sediments A) Isorenieratene (Iso) is shown in the 529.4 extracted ion chromatogram (EIC) of an authentic standard of isorenieratene B) A MS/MS spectrum taken from the starred retention time in A for the molecular mass that is starred in B confirms that this compound is isorenieratene and provides a reference mass spectrum. C) Isorenieratene and okenone (Ok) are labeled in the 529.4 EIC for the FGL sediment extract. D) A MS/MS spectrum taken from the starred retention time in $\mathrm{C}$ for the molecular mass that is starred in D confirms that this compound is isorenieratene because the retention time and mass spectrum match with those of the isolated isorenieratene depicted in panels A and B. 

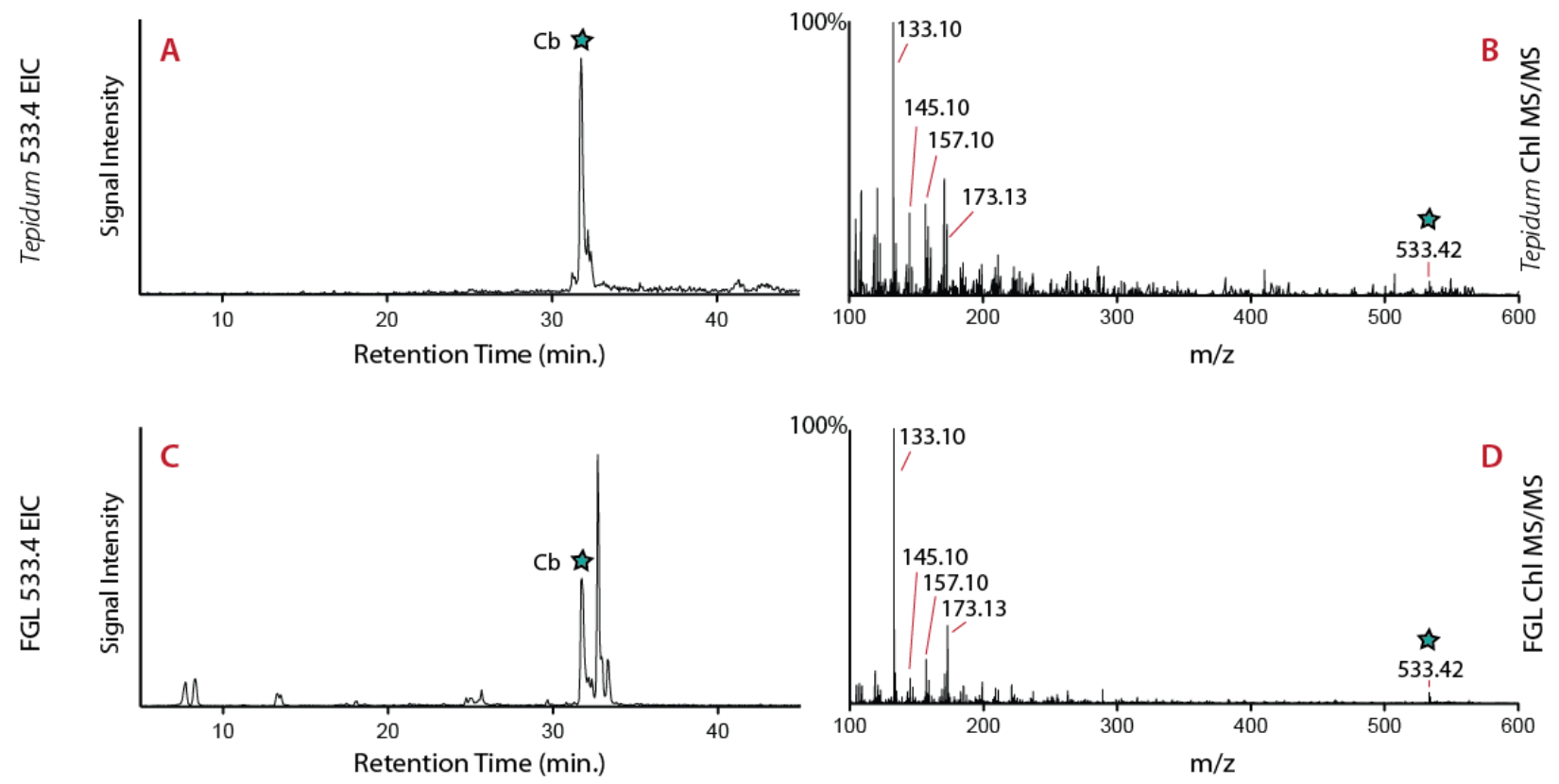

Figure 5. Identification of chlorobactene in FGL sediments $\mathrm{A})$ Chlorobactene $(\mathrm{Cb})$ is shown in the 533.4 extracted ion chromatogram (EIC) of a Chlorobium tepidum biomass extract B) A MS/MS spectrum taken from the starred retention time in A for the molecular mass that is starred in B confirms that this compound is chlorobactene and provides a reference mass spectrum. C) Chlorobactene is labeled in the 533.4 EIC for the FGL sediment extract. D) A MS/MS spectrum taken from the starred retention time in C for the molecular mass that is starred in D confirms that this compound is chlorobactene because the retention time and mass spectrum match with those of the biomass chlorobactene depicted in panels $\mathrm{A}$ and $\mathrm{B}$. 


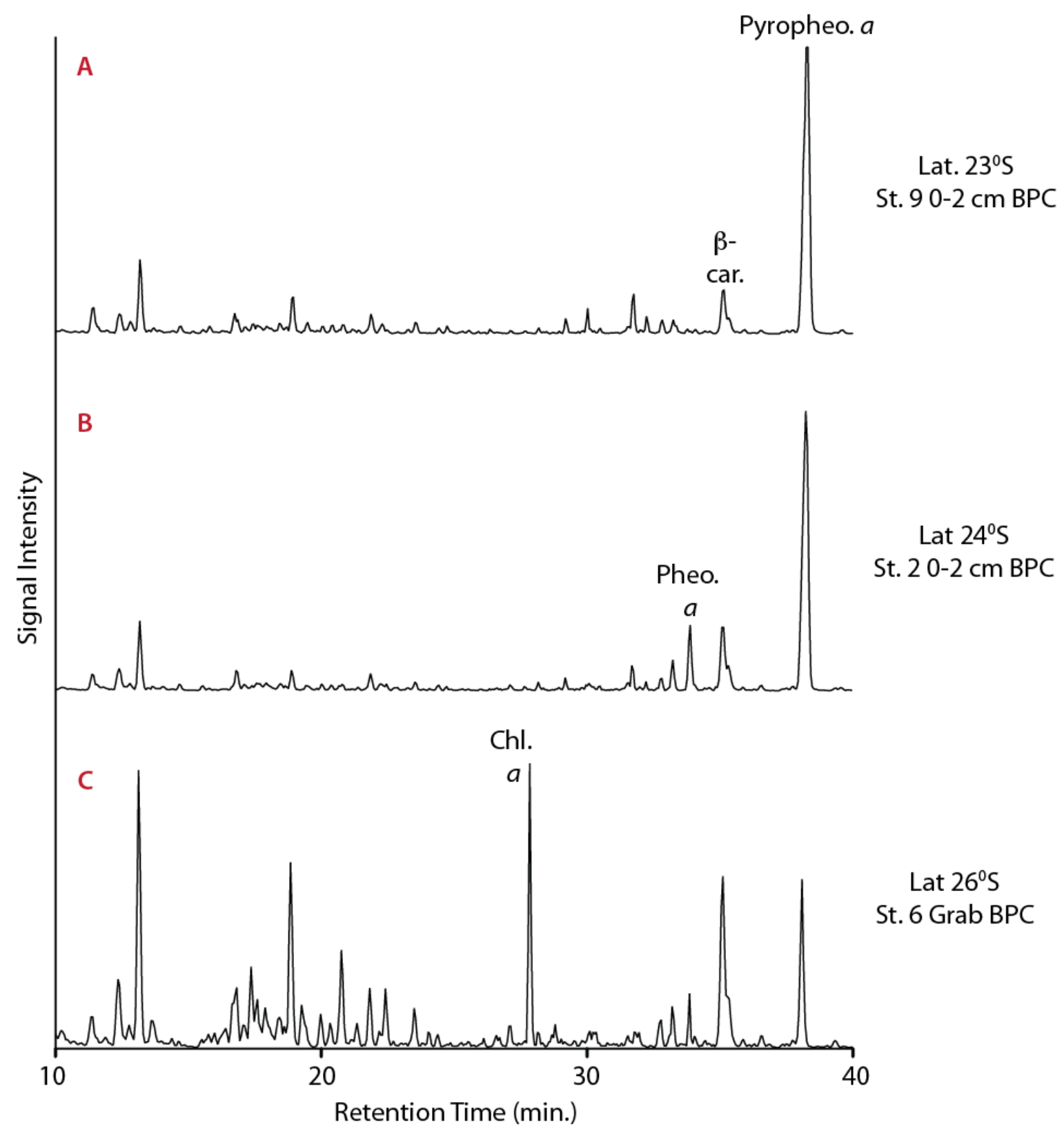

Figure 6. Pigment extracts of Namibian margin sediments. Base Peak Chromatograms (BPCs) are shown in panels A-C where the major peaks are labeled. See table 1 for sample descriptions and table 2 for peak abbreviations and identifications. 


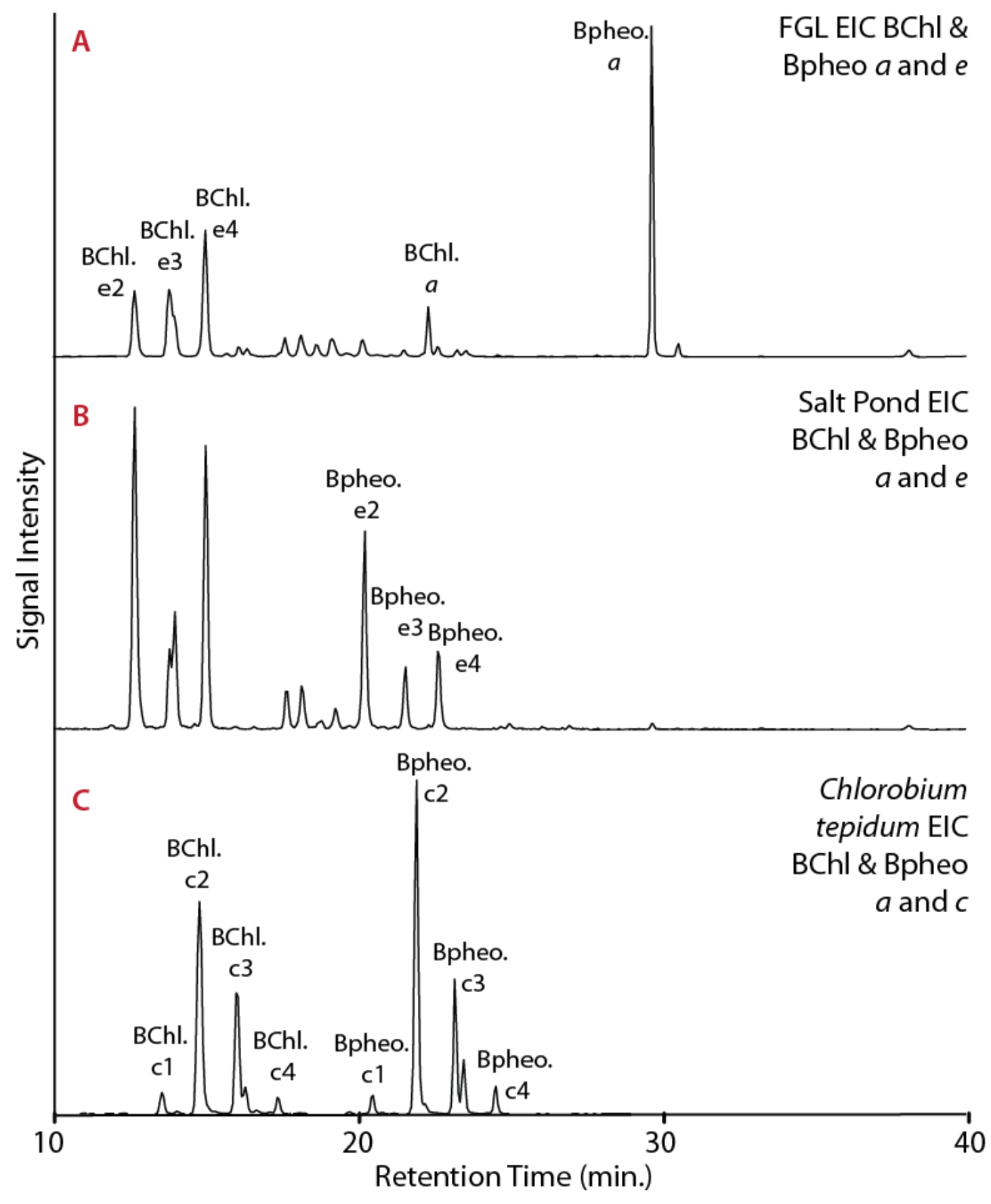

Fig. 7 Bacteriochlorophylls and bacteriopheophytins in reference material. Extracted Ion Chromatograms (EICs) are shown in panels A-C where the peaks are labeled. See table 2 for complete peak abbreviations. 
Table 1. Sample Descriptions

\begin{tabular}{|c|c|c|c|}
\hline Station \# & Station & Sample type & $\begin{array}{l}\text { Extracted freeze } \\
\text { dried mass (g) }\end{array}$ \\
\hline 1 & WW23005 & $X^{b}$ & \\
\hline 2 & WW24010 & $0-2 \mathrm{~cm}$ (core) & 2.2 \\
\hline 2 & WW24010 & $2-4 \mathrm{~cm}$ (core) & 1.8 \\
\hline 3 & WW25020 & $x$ & \\
\hline 4 & WW25005 & Grab & 5.6 \\
\hline 5 & WW26010 & $0-1 \mathrm{~cm}$ (core) & 7.4 \\
\hline 5 & WW26010 & $1-2 \mathrm{~cm}$ (core) & 5.2 \\
\hline 6 & WW26030 & Grab & 11.3 \\
\hline 7 & WW27030 & $0-2 \mathrm{~cm}$ (core) & 5.8 \\
\hline 7 & WW27030 & $2-4 \mathrm{~cm}$ (core) & 5.1 \\
\hline 8 & WW27020 & $\mathrm{X}$ & \\
\hline 9 & WW23010 & $0-2 \mathrm{~cm}$ (core) & 2.2 \\
\hline 9 & WW23010 & $2-4 \mathrm{~cm}$ (core) & 1.9 \\
\hline 10 & WW23002 & $0-2 \mathrm{~cm}$ (core) & 1.5 \\
\hline 10 & WW23002 & $2-4 \mathrm{~cm}$ (core) & 1.7 \\
\hline$X^{a}$ & Lat. $23^{\circ} \mathrm{S}$ & Natmirc grab $30 \mathrm{~m}$ & 3.6 \\
\hline
\end{tabular}

a This sample was collected on a separate monitoring expedition

b Sediment samples were not collected for these sites 
Table 2. Diagnostic fragments and identified compounds

\begin{tabular}{|c|c|c|c|c|c|c|}
\hline Compound ${ }^{a, b}$ & R. Time (min.) & $\mathbf{M +}$ & Frag. & Frag. & Ref material $^{\mathbf{b}}$ & Namibian Samples \\
\hline $\mathrm{BChl} e_{\mathrm{F}}[\mathrm{Et}, \mathrm{Et}], e 2$ & 12.6 & 821.45 & 803.44 & & FGL(40), Salt Pond(41) & $\mathrm{nd}^{\mathrm{c}}$ \\
\hline $\mathrm{BChl} c \mathrm{~F}[\mathrm{Et}, \mathrm{Me}], c 1$ & 13.6 & 793.45 & 589.27 & & Тер. $(42-44)$ & nd \\
\hline $\mathrm{BChl} e_{\mathrm{F}}[n-\mathrm{Pr}, \mathrm{Et}], e 3$ & 13.8 & 835.46 & 817.45 & & FGL, Salt Pond & nd \\
\hline $\mathrm{BChl} c \mathrm{~F}[\mathrm{Et}, \mathrm{Et}], c 2$ & 14.8 & 807.47 & 603.28 & & Tep. & nd \\
\hline $\mathrm{BChl} e_{\mathrm{F}}[i-\mathrm{Bu}, \mathrm{Et}], e 4$ & 15 & 849.48 & 831.47 & & FGL, Salt Pond & nd \\
\hline $\mathrm{BChl} c \mathrm{~F}[n-\mathrm{Pr}, \mathrm{Et}], c 3$ & 16.1 & 821.49 & 617.30 & & Tep. & nd \\
\hline $\mathrm{BChl} c_{\mathrm{F}}[i-\mathrm{Bu}, \mathrm{Et}], c_{4}$ & 17.4 & 835.50 & 631.31 & & Tep. & nd \\
\hline Bpheo $e_{\mathrm{F}}[\mathrm{Et}, \mathrm{Et}], e 2$ & 20.2 & 799.47 & 781.47 & 595.29 & FGL, Salt Pond (inferred) & nd \\
\hline Bpheo $c$ F $[\mathrm{Et}, \mathrm{Me}], c 1$ & 20.5 & 771.48 & 567.30 & & Tep. (inferred) & nd \\
\hline Bpheo $e_{\mathrm{F}}[n-\mathrm{Pr}, \mathrm{Et}], e 3$ & 21.5 & 813.49 & 795.48 & 609.31 & FGL, Salt Pond (inferred) & nd \\
\hline Bpheo $c_{\mathrm{F}}[\mathrm{Et}, \mathrm{Et}], c 2$ & 21.9 & 785.50 & 581.31 & & Tep., FGL (inferred) & nd \\
\hline BChl $a$ & 22.3 & 911.55 & 573.23 & & Std., FGL, Salt pond, Tep. & nd \\
\hline Bpheo $e_{\mathrm{F}}[i-\mathrm{Bu}, \mathrm{Et}], e 4$ & 22.6 & 827.51 & 809.50 & 623.32 & FGL, Salt Pond (inferred) & nd \\
\hline Bpheo $c_{\mathrm{F}}[n-\mathrm{Pr}, \mathrm{Et}], c 3$ & 23.2 & 799.52 & 595.33 & & Tep., FGL (inferred) & nd \\
\hline Chl $b$ & 23.8 & 907.52 & 569.20 & 629.22 & Spinach, FGL & nd \\
\hline Bpheo $c_{\mathrm{F}}[i-\mathrm{Bu}, \mathrm{Et}], c 4$ & 24.6 & 813.53 & 609.34 & & Tep., FGL (inferred) & nd \\
\hline Okenone & 24.8 & 579.42 & 133.10 & 547.39 & FGL, Salt Pond & nd \\
\hline Chl $a$ & 27.7 & 893.54 & 614.24 & 555.22 & Spinach, FGL, Salt Pond & Yes \\
\hline Chl $a^{\prime}$ epimer & 28.8 & 893.54 & 614.24 & 555.22 & Spinach, FGL, Salt Pond & Yes \\
\hline Bpheophytin $a$ & 29.6 & 889.58 & 611.29 & 551.27 & Std., FGL, Salt Pond & nd \\
\hline Isorenieratene & 30.7 & 529.38 & 133.10 & 173.13 & Isolated Ref. (D. Repeta) & nd \\
\hline Isorenieratene isomer & 31.4 & 529.38 & 133.10 & 173.13 & Isolated Ref. (D. Repeta) & nd \\
\hline Chlorobactene & 31.8 & 533.41 & 133.10 & 173.13 & Tep. & nd \\
\hline Bpyropheophytin $a$ & 32 & 831.58 & 552.27 & & FGL, Salt Pond (inferred) & nd \\
\hline Pyropheophytin $b$ & 33.2 & 827.547 & 549.25 & 521.24 & inferred & Yes (tentative) \\
\hline Pheophytin $a$ & 34 & 871.57 & 593.28 & 533.25 & Spinach, FGL, Salt Pond & Yes \\
\hline Pheophytin $a^{\prime}$ epimer & 34.9 & 871.57 & 593.28 & 533.25 & Spinach, FGL, Salt Pond & Yes \\
\hline B-carotene & 35.1 & 537.44 & 177.16 & & Std., Spinach, FGL & Yes \\
\hline Pyropheophytin $a$ & 38.2 & 813.57 & 535.27 & 507.25 & FGL, Salt Pond (inferred) & Yes \\
\hline
\end{tabular}

a [8-R,12-R] are the positions of the alkyl substitutions

b Abbreviations: Subscript F: farnesyl esterified; Chl: chlorophyll; pheo: pheophytin; B: bacterio; Tep.: Chlorobium tepidum; Std.: Purchased standard c nd is "not detected" 


\section{Chapter 6:}

Diagenetic and detrital origin of moretane anomalies through the Permian-Triassic boundary

This chapter was published in Geochimica et Cosmochimica Acta (GCA) by Elsevier in 2012 and is reprinted here with their permission as granted in the original copyright agreement.

French KL, Tosca NJ, Cao C, Summons RE (2012) Diagenetic and detrital origin of moretane anomalies through the Permian-Triassic boundary. Geochim Cosmochim Acta 84:104-125. 


\title{
Diagenetic and Detrital Origin of Moretane Anomalies through the Permian-Triassic Boundary
}

French, Katherine L. ${ }^{a}$ Tosca, Nicholas J. ${ }^{b}$ Cao, Changqun ${ }^{c}$ Summons, Roger E. ${ }^{d}$

a Joint Program in Chemical Oceanography, Massachusetts Institute of Technology and Woods Hole Oceanographic Institution, Cambridge, Ma 02139, United States

${ }^{b}$ Department of Earth Sciences, University of St Andrews, St Andrews, KY16 9AL, Scotland UK

cState Key Laboratory of Palaeobiology and Stratigraphy, Nanjing Institute of Geology and Palaeontology, Chinese Academy of Sciences, Nanjing, Jiansu, 210008, China

${ }^{\mathrm{d}}$ Department of Earth, Atmospheric, and Planetary Sciences, Massachusetts Institute of Technology, Cambridge, Ma 02139, United States

\begin{abstract}
Many biogeochemical anomalies coincide with the Late Permian Extinction (LPE; 252.28 Ma). Several mechanisms have been proposed to explain the moretane/hopane anomaly that has been identified in samples from Meishan GSSP section in southeastern China. Here, we report homohopane, $2 \alpha$ - and $3 \beta$-methylhomohopane and lithological data for a drill core from the Meishan section in southeastern China. Three intervals of elevated $\mathrm{C}_{30}$ moretane/hopane ratios are recorded in the Lungtan, Yinkeng and Helongshan Formations. Moretane/hopane ratios of $\mathrm{C}_{31-34}$ homohopanes and the $2 \alpha$ - and $3 \beta$-methylhomohopanes display the same stratigraphic patterns as the $\mathrm{C}_{30}$ moretane/hopane record. In light of the multiple and parallel moretane anomalies for the homohopane and $2 \alpha$ - and $3 \beta$-methylhomohopane series, enhanced input from higher plant organic matter, such as coal and peat, does not adequately explain the observed isomer patterns. Correlation of high moretane/hopane ratios with low $\mathrm{C}_{35}$ Homohopane Index (HHI) and high hopane/sterane values suggest increased input of
\end{abstract}


hopanoids from oxic soils. Additionally, moretane/hopane ratios show excellent correlations with total clay percentages and specific clay types, particularly chlorite, illite, and mixed layer illite/smectite. We conclude that a combination of episodic hopanoid input from soil bacteria and diagenetic effects related to redox and detrital clays generated the unique moretane/hopane patterns at Meishan.

Similar relationships of $\mathrm{Ts} /(\mathrm{Ts}+\mathrm{Tm})$ with redox and source indicators and lithology indicate that $\mathrm{Ts} /(\mathrm{Ts}+\mathrm{Tm})$ is affected by the same factors controlling the moretane/hopane ratios. Berthierine, a clay that requires reducing conditions for formation, was detected in samples from the Lungtan Formation. We are unable to determine from our results whether the berthierine is authigenic or detrital, but future determination of the origin of berthierine at Meishan may offer additional environmental insight. No link between diasteranes and lithology was observed in this study suggesting that diasteranes are relatively unaffected by the detrital clay component of the Meishan sediments. In total, the results point toward the complex role of source input, lithology, and depositional redox conditions in the transformation of organic matter during maturation. Future work is required to elucidate the lithological effects on diagenetic processes, including biomarker genesis isomerization, and thermal degradation.

Key words: Late Permian Extinction; Meishan Section; hydrocarbon biomarkers; hopanes; moretanes; clay composition; diagenesis; berthierine 


\subsection{INTRODUCTION}

Through the course of Earth's 4.5 billion year history, there have been five mass extinction events in which at least $75 \%$ of species became extinct in a geologically short time interval. Given the rapid loss of biodiversity over the past centuries, some researchers have suggested that Earth may currently be entering its sixth mass extinction event (Barnosky et al., 2011). Humans are suspected to have a role in today's loss of biodiversity, but other conditions and series of events must have caused the previous five mass extinctions. The Late Permian Extinction (LPE; 252.28 Ma) (S. Shen et al., 2011), which occurred shortly before the biostratigraphic Permian Triassic Boundary (PTB) as defined by the first appearance of Hindeodus parvus, marks the greatest loss of biodiversity in Earth history with over $90 \%$ of marine species becoming extinct (Raup, 1979; Mundil et al., 2001, 2004; Sepkoski, 2002; Erwin, 2006; Metcalfe and Isozaki, 2009). Many mechanisms have been proposed to trigger the LPE event, including Siberian flood basalt volcanism, sea level change, extraterrestrial impact, ocean deoxygenation, water column stratification and climate change driven by methane hydrate collapse (Campbell et al., 1992; Wignall and Hallam, 1992; Renne et al., 1995; Becker et al., 2001; Benton and Twitchett, 2003; Kamo et al., 2003). Whatever the trigger or combination of triggers, accumulating evidence points to the rapid deterioration of both the marine and terrigenous ecosystems (Retallack, 1995; Looy et al., 2001; Twitchett et al., 2001; Michaelsen, 2002).

Geochemical techniques have helped to elucidate the nature of the events that unfolded during the Late Permian, yet additional questions have arisen as a result. In addition to a distinctive negative stable carbon isotopic excursion and trace metal

enrichment at the boundary (Kaiho et al., 2001, 2006; Cao et al., 2002, 2009), anomalous distributions of lipid biomarkers, including isorenieratane and derived aryl isoprenoids, 2-methylhopanes and crocetane, are defining features of the PTB (Grice et al., 2005; 
Wang et al., 2005; Xie et al., 2005; Wang, 2007; Cao et al., 2009). Additionally, anomalous

values of molecular ratios, including $\mathrm{C}_{30} 17 \beta, 21 \alpha(\mathrm{H})$-hopane/ $\mathrm{C}_{30} 17 \alpha, 21 \beta(\mathrm{H})$-hopane (referred to as the $\mathrm{C}_{30}$ moretane/hopane ratio), $\mathrm{C}_{27} 18 \alpha$-trisnorhopane/ $\mathrm{C}_{27} 17 \alpha$ trisnorhopane (referred to as $\mathrm{Ts} /(\mathrm{Ts}+\mathrm{Tm})$ ), $\mathrm{Tm} / \mathrm{C}_{30}$ hopane, $\mathrm{C}_{31} / \mathrm{C}_{32}$ hopane, $\mathrm{C}_{35}$ Homohopane Index $\left(\mathrm{C}_{35} \mathrm{HHI}\right)$, and hopane/sterane, are recorded in sediments deposited at the PTB (Wang, 2007; Xie et al., 2007; Cao et al., 2009).

Multiple scenarios have been proposed to explain the elevated moretane/hopane ratios at the PTB. Geochemical maturity parameters throughout the Meishan GSSP section in southeastern China are nearly uniform, and thus, thermal maturity variations cannot adequately explain the moretane/hopane and $\mathrm{Ts} /(\mathrm{Ts}+\mathrm{Tm})$ variability observed through the section (Cao et al., 2009). Accordingly, additional mechanisms have been proposed to account for the moretane variability, including increased input of higher plant organic matter to the marine system, increased acidification, freshening of the upper water column, or lithological effects (Wang, 2007; Xie et al., 2007; Cao et al., 2009). In order to further assess and correctly interpret the moretane anomalies at the PTB, homohopane, $2 \alpha$ - and $3 \beta$-methylhomohopane and lithological data for a drill core taken from the Meishan section in China were critically examined. The possible mechanisms for generating the observed moretane record, including thermal maturity, source input, redox conditions, and lithology, were examined in light of the new hopane and lithological data.

\subsubsection{Moretane Background}

Hopanes are pentacyclic triterpanes and are arguably the most ubiquitous natural product found on Earth (Ourisson and Albrecht, 1992). Bacteria are the dominant source of hopanes in marine sediments, and bacteriohopanepolyols (BHP) are the primary precursor of $C_{30}$ and higher hopane homologues. Hopanes have readily isomerized asymmetric carbon atoms at C-17 and C-21, where $\alpha$ and $\beta$ denote whether 
the hydrogen is below or above the plane of the ring system, respectively. Consequently, when considering C-17 and C-21, there are four possible stereoisomers (Fig. 1): $\quad 17 \beta, 21 \beta(\mathrm{H})$-hopane $\quad(\beta \beta$-hopane), $17 \beta, 21 \alpha(\mathrm{H})$-hopane $\quad(\beta \alpha$-moretane), $17 \alpha, 21 \beta(\mathrm{H})$-hopane ( $\alpha \beta$-hopane), and $17 \alpha, 21 \alpha(\mathrm{H})$-hopane ( $\alpha \alpha$-hopane; this isomer is not generally encountered in sediments). The term "moretane" distinguishes the $17 \beta, 21 \alpha(\mathrm{H})$-hopane stereoisomers from the other hopane stereoisomers, while the others are simply referred to as 'hopanes'. The $\beta \beta$-hopanoids are the commonly observed biological configuration and are found in bacterial cultures and immature organic material. The $\beta \beta$ configuration is nearly planar, which enables the molecule to fit into the membrane lipid bilayer (Peters et al., 2005), but the $\beta \beta$-hopane is the least thermodynamically stable of the hopane stereoisomeric series (Seifert and Moldowan, 1980; Kolaczkowska et al., 1990; Peters et al., 2005). During diagenesis and catagenesis, $\beta \beta$-hopane is removed by thermal degradation or interconversion to the more thermodynamically stable $\beta \alpha$-moretane and $\alpha \beta$-hopane. According to the scheme illustrated in Seifert and Moldowan (1980), $\beta \alpha$-moretane can overcome an energy barrier given sufficient thermal energy to be converted to the thermodynamically preferred $\alpha \beta$-hopane via the $\beta \beta$-hopane intermediate (Peters et al., 2005). The $\alpha \alpha$ hopane is less thermodynamically stable than either $17 \beta, 21 \alpha(\mathrm{H})$-moretane or $17 \alpha, 21 \beta(\mathrm{H})$-hopane, and it is largely undetected in petroleum and mature petroleum source rocks (Bauer et al., 1983; Kolaczkowska et al., 1990).

The distributions of terpane isomers can be used to estimate thermal maturity. Predictions of thermodynamic stability of the different hopane stereoisomeric series and observations of the degree of isomerization of hopanes in immature organic matter and petroleum have led to use of the $\beta \alpha$-moretane to $\alpha \beta$-hopane ratio as a thermal maturity indicator (Seifert and Moldowan, 1980; Peters et al., 2005). The $\beta \alpha$-moretane/ $\alpha \beta$-hopane ratio is used to characterize immature to mildly mature oils. However, thermal maturity is not the only factor that influences the $\beta \alpha$-moretane/ $\alpha \beta$-hopane ratio. The source of 
organic matter input and depositional environment influence the terpane fingerprint of petroleum and can significantly affect thermal maturity parameters (Peters et al., 2005). Based on reports of $17 \beta, 21 \alpha(\mathrm{H})$ hopanoids in hypersaline environments, peat, coal, lacustrine environments, and in living systems (Quirk et al., 1984; Rullkötter and Marzi, 1988; Uemura and Ishiwatari, 1995; Rosa-Putra et al., 2001), some authors have invoked increased input of higher plant organic matter to explain anomalous moretane abundance in marine sediments where thermal maturity is not the cause of variability (Grantham, 1986; Isaksen and Bohacs, 1995; Wang, 2007; Xie et al., 2007). However, in a number of instances, depositional environment has been shown to strongly modulate terpane distributions, including the abundance of moretane relative to $\alpha \beta$-hopane (Peters et al., 2005).

\subsection{SAMPLES AND EXPERIMENTAL METHODS}

\subsubsection{Geographic Setting}

The Meishan quarries in South China have produced detailed information about the Permian-Triassic mass extinction. The Meishan section is the Global Stratotype Section and Point (GSSP) for the PTB, as well as for the base of Changhsingian Stage. In order to avoid sampling material that was altered and contaminated during surficial weathering, a drilling project was undertaken by the Nanjing Institute of Geology and Paleontology in 2004. A detailed description of the Meishan section and the drilling project are provided in the supplementary online material of Cao et al. (2009).

\subsubsection{Biomarker Analyses}

As described by Cao et al. (2009), the exterior of the samples were brushed clean and rinsed with methanol $(\mathrm{MeOH})$ and dichloromethane $(\mathrm{DCM})$ prior to being powdered using a solvent-cleaned ceramic puck mill. Procedural blanks were prepared 
along with the samples and showed no evidence of laboratory contamination. Samples were solvent extracted using a Dionex ASE-200 extractor and a solvent mixture of DCM and $\mathrm{MeOH}$ (9:1). Elemental sulfur was removed from the total lipid extracts using acidwashed copper granules. Aliphatic, aromatic, and polar fractions were collected by silica column chromatography using hexane, hexane/DCM (4:1), and DCM/MeOH (4:1) solvents, respectively.

Each fraction was dried and weighed before adding analytical standards to $1 \mathrm{mg}$ of the saturated fraction. The saturated hydrocarbon biomarkers were analyzed by gas chromatography-mass spectrometry (GC-MS) in full scan and metastable reaction monitoring (MRM) modes. The characteristic $m / z 191$ and 205 mass fragments were used to identify the $\mathrm{C}_{30}-\mathrm{C}_{34}$ homohopanes and $2 \alpha$ - and $3 \beta$ - methylhopanes in MRM mode, respectively (see Fig. 2). The $22 \mathrm{~S}$ and $22 \mathrm{R}$ isomers of $\mathrm{C}_{31-34}$ homohopanes and $\mathrm{C}_{32}-$ 33 methylhomohopanes were identified, and both isomers were used in the calculation of the $\beta \alpha /(\beta \alpha+\alpha \beta)$ ratios. The $22 \mathrm{~S}$ and $22 \mathrm{R}$ isomers of $\beta \alpha-\mathrm{C}_{31}$ homohopane and $\beta \alpha-\mathrm{C}_{32}$ methylhomohopane coelute, so these compounds were integrated as a single peak as illustrated in figure 2. The supplementary online material of Cao et al. (2009) describes the biomarker analytical methods in greater detail.

\subsubsection{Mineralogical Analyses}

\section{Carbonate Contents}

Approximately $0.5 \mathrm{~g}$ of each powdered sample was accurately weighed into a clean Teflon tube. Methanol was added to wet the sample prior to acidification. Each sample was acidified with a $10 \%$ aqueous hydrochloric acid $(\mathrm{HCl})$ solution. The samples were treated again with a $15 \% \mathrm{HCl}$ solution and left for 24 hours to allow the reaction to reach completion. The samples were rinsed with water five times to remove any remaining acid before being dried in a $60^{\circ} \mathrm{C}$ oven for 48 hours. The samples were 
reweighed, and the percentage carbonate was calculated based on the difference between the initial and final weights.

\section{Clay analyses}

Preparation for bulk mineralogical analysis of all samples involved crushing rock samples in an agate swing mill, addition of $10 \mathrm{wt} . \% \mathrm{ZnO}$ and further milling to ensure mixing and homogeneous distribution. Bulk mineralogical analysis was performed on randomly oriented samples using a Bruker D8 X-ray diffractometer at $40 \mathrm{kV}$ and $30 \mathrm{~mA}$ with $\mathrm{CuK} \alpha$ radiation. Analyses were performed from $2-65^{\circ} 2 \mathrm{Q}$ (for any diffracted X-ray obeying Bragg's Law, 2Q as referred to herein is equal to 2 times the angle between the sample plane and the incident X-ray beam) at a step size of 0.02 degrees and counting times of 4 seconds per step. Quantitative analysis was performed with single line and full profile fitting using pure mineral standards for reference intensity ratios (Srodon et al., 2001).

Determination of clay mineralogy involved light crushing of samples by hand in a steel mortar and pestle and for samples containing appreciable carbonate content, decarbonation with $1 \mathrm{M}$ acetic acid while monitoring $\mathrm{pH}$. Decarbonated samples were rinsed three times with deionised water and all samples were resuspended with sodium phosphate, sonicated and the $<2$ and $<0.2 \mathrm{~mm}$ size fractions were obtained by timed centrifugation. Centrifuged samples were decanted and oriented Ca-saturated aggregates were prepared by using a filter membrane technique and transfer of clay films for glass substrates. X-ray diffraction was performed using a Siemens D5000 diffractometer at $30 \mathrm{kV}$ and $20 \mathrm{~mA}$, with $\mathrm{CuK} \alpha$ radiation from $2-35^{\circ} 2 \mathrm{Q}$ a step size of 0.03 degrees and counting times of 8-10 seconds. Divergence, receiving and anti-scatter slits were 0.2 and $1 \mathrm{~mm}$ in size, respectively, and a Ni filter was used. Clay samples were analysed in the air-dried Ca-saturated state, after ethylene glycol solvation overnight at $60^{\circ} \mathrm{C}$ (to identify expandable minerals), heating at $400^{\circ} \mathrm{C}$ for 2 hours (to quantify 
collapsible/expanding mineral proportions), and heating to $550^{\circ} \mathrm{C}$ for 2 hours (to aid in quantifying kaolinite and chlorite proportions). Relative clay abundances were determined by peak area measurement, expressed in relative percent and normalized to total clay content by total clay mineral abundance determined by bulk mineralogical analyses. The composition of mixed-layered species was determined using one dimensional X-ray diffraction pattern modeling with the software package NEWMOD (Reynolds and Reynolds, 1996).

\subsection{RESULTS}

\subsubsection{Stratigraphic Variation of Lithology and Hopane Distributions}

The $\beta \alpha /(\beta \alpha+\alpha \beta)$ ratios for $\mathrm{C}_{30}$ hopane, $\mathrm{C}_{31-34}$ homohopanes, $\mathrm{C}_{31-33} 2 \alpha-$ methylhopanes, and $\mathrm{C}_{31-33} 3 \beta$-methylhopanes were calculated for each sample (Table 1). The precise determination of $\beta \alpha /(\beta \alpha+\alpha \beta)$ for the $C_{31}$ and $C_{32} 3 \beta$-methylhopanes was precluded by interfering peaks and will not be further discussed. The $\beta \alpha /(\alpha \beta+\beta \alpha)$ values vary from 0.04 to 0.34 for all of the other hopane series measured. The profiles of $\mathrm{C}_{30}$ hopane, the homohopanes, and the $2 \alpha$ - and $3 \beta$-methylhomohopanes show parallel downcore trends and similar values (Fig. 3). Ts/(Ts+Tm) and $\mathrm{C}_{35}$ Homohopane Index (HHI) data from Cao et al., 2009 were plotted in figure 3 for comparison. Notably, the rocks record three positive moretane enrichments. The first interval of enhanced moretane/hopane ratios occurs in the Lungtan Formation, followed by the second interval at the PTB in the Yinkeng Formation, and the last interval of moretane enrichment at the end of the Griesbachian in the Helongshan Formation.

The bulk lithology, including percent carbonate, total clay, and quartz was determined (Table 2). The lithology was highly variable through the drill core section (Fig. 4). The percent carbonate fluctuates from 8.5 to $99 \%$. The percent total clay and the percent quartz both vary from close to $0 \%$ to nearly $50 \%$. The Lungtan Formation is a 
clay-rich unit that is enriched in quartz and is characterized by low carbonate ( 8.5 to $14 \%)$. The percent carbonate increases significantly in the overlying Changxing unit, although the carbonate and quartz percentages are more variable through this interval. Unlike the Lungtan Formation, clay is a minor component through the Changxing Formation. The percent total clay returns to elevated values in the Yinkeng Formation, while the percent carbonate is significantly lower than the Changxing or Helongshan formations. Through the Helongshan Formation, the samples are all over 95\% carbonate with very little clay or quartz. Similar to the observations of Rullkötter and Marzi (1988), elevated abundances of moretanes relative to $\alpha \beta$-hopane are associated with carbonate-poor facies.

Further clay mineralogical analyses provided the absolute and relative percentages of specific clay types, including illite, chlorite, smectite, kaolinite, berthierine, and mixed layer illite/smectite (Table 3). The total percent clay and the absolute and relative percentages of the clay type varied through the section (see Fig. 5). Illite and mixed layer illite/smectite represent the dominant clay types, where the sum of percent illite and mixed layer illite/smectite represents over $50 \%$ through the entire section. The relative percentage of illite/smectite mixed layer clay is largely constant throughout the clay mineral assemblage. Although more variable, kaolinite, chlorite, and smectite clays become a significant fraction of the total clay through some intervals of the assemblage. Notably, berthierine, which is usually formed under reducing conditions (Taylor and Curtis, 1995; Fritz and Toth, 1997), is present in the Lungtan Formation at the base of the section. Measurable differences between the glycolated and heated $\left(400^{\circ} \mathrm{C}\right)$ samples in the $7 \AA$ peak suggest that trace abundances of berthierine may be present in the samples between $92-103 \mathrm{~m}$. It is not possible to determine from our results whether the berthierine is detrital or authigenic. 


\subsubsection{Crossplots of Molecular Indices and Lithological Data}

Cross-correlation diagrams were generated to evaluate the relationships between the geochemical and lithological data. The $\beta \alpha /(\alpha \beta+\beta \alpha)$ ratios of $C_{30}$ hopane were plotted against the $\beta \alpha /(\alpha \beta+\beta \alpha)$ ratios of $\mathrm{C}_{31}-\mathrm{C}_{34}$ homohopanes and the $2 \alpha$ and $3 \beta$ methylhomohopanes (Fig. 6A and B). In both cases, an excellent positive correlation is observed with $\mathrm{R}^{2}$ values ranging from 0.88 to 0.99 . Cao et al. (2009) found that $\mathrm{Ts} /(\mathrm{Ts}+\mathrm{Tm})$ varied inversely with the $\mathrm{C}_{30}$ hopane $\beta \alpha /(\alpha \beta+\beta \alpha)$ ratio. Likewise, the $\mathrm{Ts} /(\mathrm{Ts}+\mathrm{Tm})$ data reported by Cao et al. (2009) also correlate inversely with the homohopane and $2 \alpha$ - and $3 \beta$ - methylhomohopane $\beta \alpha /(\alpha \beta+\beta \alpha)$ ratios with $\mathrm{R}^{2}$ values ranging from 0.64 to 0.77 (Fig. $6 \mathrm{C}$ and D).

The carbonate percentage and total clay percentage were plotted against the moretane/hopane ratios for all of the hopane series and Ts/(Ts+Tm) (Fig. 7). The percent carbonate and percent clay have opposite relationships with the $\beta \alpha /(\alpha \beta+\beta \alpha)$ ratios. Carbonate percentage and the $\beta \alpha /(\alpha \beta+\beta \alpha)$ ratios of all of the hopane series are inversely related, having $R^{2}$ values ranging from 0.42 to 0.57 . The correlation is significantly improved with $R^{2}$ values from 0.74 to 0.87 when the $\beta \alpha /(\alpha \beta+\beta \alpha)$ ratios are plotted against total clay percentage. Similarly, the correlation of $\mathrm{Ts} /(\mathrm{Ts}+\mathrm{Tm})$ with the total clay percentage $\left(R^{2}=0.52\right)$ is stronger than with the percent carbonate $\left(R^{2}=0.36\right)$. However, the $\mathrm{R}^{2}$ values for $\mathrm{Ts} /(\mathrm{Ts}+\mathrm{Tm})$ versus carbonate and total clay percentages are less than for the $\beta \alpha /(\alpha \beta+\beta \alpha)$ ratios in both cases.

The $\beta \alpha /(\alpha \beta+\beta \alpha)$ ratios for all of the hopane series were plotted together with the percentages of the different clay types in Fig. 8. Like the total clay percentage, all of the clay types were directly related to $\beta \alpha /(\alpha \beta+\beta \alpha)$, yet some clay types were more strongly correlated to $\beta \alpha /(\alpha \beta+\beta \alpha)$ than others. Kaolinite had the weakest correlation with $\beta \alpha /(\alpha \beta+\beta \alpha)$, having $\mathrm{R}^{2}$ values from 0.024 to 0.222 . Likewise, smectite was weakly correlated to $\beta \alpha /(\alpha \beta+\beta \alpha)$, having $\mathrm{R}^{2}$ values spanning 0.215 to 0.485 . On the other hand, the percent chlorite was strongly correlated with $\beta \alpha /(\alpha \beta+\beta \alpha)$, where $\mathrm{R}^{2}$ values ranged 
from 0.773 to 0.901 . Percent illite and percent mixed layer illite/smectite also yielded strong correlations with $\beta \alpha /(\alpha \beta+\beta \alpha)$.

The Ts/(Ts+Tm) values from Cao et al. (2009) were plotted versus the different types of clays. Low values of $\mathrm{Ts} /(\mathrm{Ts}+\mathrm{Tm})$ occur during intervals of high clay accumulation, in particular, intervals where chlorite, illite, and illite/smectite mixed layer clays comprise a higher proportion of rock lithology. The $\mathrm{R}^{2}$ values for $\mathrm{Ts} /(\mathrm{Ts}+\mathrm{Tm})$ were less than the $R^{2}$ values for the analogous $\beta \alpha /(\alpha \beta+\beta \alpha)$ plots. Like $\beta \alpha /(\alpha \beta+\beta \alpha)$, kaolinite and smectite produced the weakest correlation coefficients, while illite, chlorite, and mixed layer illite/chlorite had higher correlation coefficients with $\mathrm{Ts} /(\mathrm{Ts}+\mathrm{Tm})$.

\subsubsection{Crossplots of $\mathrm{C}_{35}$ Homohopane Index and Hopane/Sterane ratios with Moretane/Hopane, Ts/(Ts+Tm) and Lithological Data}

The $\mathrm{C}_{35} \mathrm{HHI}$ is a redox indicator, and redox conditions are known to affect the distribution of terpanes (Peters et al., 2005). Cross-correlation diagrams were generated to evaluate the relationships between $\mathrm{C}_{35} \mathrm{HHI}$ data reported in Cao et al., 2009 and moretane/hopane ratios and Ts/(Ts+Tm) (Fig. 9). High moretane/hopane ratios for $\mathrm{C}_{30-34}$ homohopanes and $\mathrm{C}_{31-33}$ methylhomohopanes correspond to low values of $\mathrm{C}_{35} \mathrm{HHI}$, which are indicative of oxic conditions. On the other hand, low values of Ts/(Ts+Tm) correspond with low values of $\mathrm{C}_{35} \mathrm{HHI}$. These results are consistent with previous results relating redox indicators to moretane hopane ratios and $\mathrm{Ts} / \mathrm{Ts}+\mathrm{Tm}$ ) (Moldowan et al., 1986; Rullkötter and Marzi, 1988; Wang, 2007). Interestingly, high values of hopane/sterane percentages reported in Cao et al., 2009 correspond with high values of moretane/hopane ratios for $\mathrm{C}_{30-34}$ homohopanes and $\mathrm{C}_{31-33}$ methylhomohopane and low values of Ts/(Ts+Tm) (Fig. 9). This relationship is unexpected because if the abundance

of $\alpha \beta$-hopanes were driving the correlation between hopane/sterane ratios and 
moretane/hopane ratios, one would expect an inverse pattern between these two parameters, which is the opposite of what is observed.

The $\mathrm{C}_{35} \mathrm{HHI}$ and hopane/sterane percentages were plotted against total clay percent and all of the clay types detected in the Meishan section (Fig. 10 and 11). In

general, the highest values of percent total clay and individual clay types correspond to $\mathrm{C}_{35} \mathrm{HHI}$ values less than $5 \%$, but several samples with significant kaolinite abundances have higher $\mathrm{C}_{35} \mathrm{HHI}$ values. High values of hopane/sterane percentages are related to high values of total clay, chlorite, illite, mixed layer illite/smectite (I/S), and smectite. Kaolinite and berthierine do not display this same relationship with the hopane/sterane percentages (see Fig. 11F and 11G).

\subsection{DISCUSSION}

\subsubsection{Possible Causes of Moretane Enrichment}

The strong positive correlations between the $C_{30}$ hopane $\beta \alpha /(\alpha \beta+\beta \alpha)$ ratios and the homohopane and $2 \alpha$ - and $3 \beta$-methylhomohopane $\beta \alpha /(\alpha \beta+\beta \alpha)$ ratios imply that the moretane/hopane ratios for all of the hopane series are controlled by the same mechanism, and the mechanism that caused the anomalously high moretane values is not confined or unique to the extinction horizon. Our results show that moretanes are enriched in rocks that are clay-rich and have $\mathrm{C}_{35} \mathrm{HHI}$ values indicating oxic conditions, which is consistent with the results of Wang, (2007) and Rullkötter and Marzi, (1988). Thermal maturity, the source of organic matter and depositional environment are the three possible factors that can influence the distribution of triterpane isomers (Peters et al., 2005). One or more of these factors is likely responsible for generating the moretane excursions recorded in the Lungtan, Yinkeng and Helongshan formations.

Thermal maturity can be estimated by measuring the degree of isomerization, including the ratio of $\beta \alpha$-moretanes to $\alpha \beta$-hopanes, the conversion of $22 \mathrm{R}$ to $22 \mathrm{~S}$ for $17 \alpha$, 
$21 \beta(\mathrm{H})$-homohopanes and the conversion of $20 \mathrm{R}$ to $20 \mathrm{~S}$ steranes. The moretane to $\alpha \beta-$ hopane ratio for the $\mathrm{C}_{30}$ compound declines with increasing thermal maturity from $\sim 0.8$ in immature rocks to $<0.15$ for mature rocks (Peters et al., 2005). While the ratio of moretanes to $\alpha \beta$-hopanes varies significantly through the drill core, the ratio of $22 \mathrm{~S} /(22 \mathrm{~S}+22 \mathrm{R})$ for $\mathrm{C}_{31}$ homohopane is between 54 and $59 \%$ throughout the entire Meishan drill core (Cao et al., 2009). According to Larcher et al. (1987), these values are expected for $\alpha \beta$-hopanes when the epimerization reaction that converts the biological $22 \mathrm{R}$ epimer to $22 \mathrm{~S}$ has reached endpoint. The epimerization end point is met before the main phase of oil generation (Peters et al., 2005). The 20S/(20S+20R) ratios of the $C_{27}$ steranes varies between $45-50 \%$, which further supports the conclusion that the whole cored interval is within the early stages of petroleum generation and shows little variation throughout (Cao et al., 2009). The largely constant relative percent of illite/smectite mixed layer clay in the section is also consistent with constant thermal maturation throughout the Meishan drill core (Pollastro, 1993). Accordingly, thermal maturity can be ruled out as the factor responsible for moretane variability. Likewise, Wang (2007) and Xie et al. (2007) eliminated thermal maturity as a possible mechanism for generating the moretane anomaly based on additional lines of evidence.

The sources of organic matter profoundly affect biomarker distributions (Peters et al., 2005). Previous studies have argued that increased terrigenous organic matter input from higher plants, peat, or coal explains elevated moretane/hopane ratios in cases where thermal maturity does not sufficiently explain the pattern of moretane/hopane ratios (Grantham, 1986; Rullkötter and Marzi, 1988; Isaksen and Bohacs, 1995). The link between moretanes and higher plant organic matter input is based on a series of studies that detected elevated $\beta \alpha$-hopanoids in terrigenous influenced sediments and some higher plants. Unusually high abundances of $17 \beta(\mathrm{H})$, $21 \alpha$ - moretan-29-ol and $17 \beta(\mathrm{H})$-moret-22(29)-ene have been reported in acidic and saline lakes (Uemura and Ishiwatari, 1995; Ishiwatari et al., 2005; Aichner et al., 2010; 
Kristen et al., 2010). Quirk et al. (1984) detected $C_{32} \beta \alpha$-alcohols, $C_{31}$ methylmoretan-29$\mathrm{ol}$, and $\mathrm{C}_{32}$ and $\mathrm{C}_{33} \beta \alpha$ hopanoic acids in peat samples, and elevated levels of moretane were reported for coals (e.g., Hughes and Dzou, 1995; Shen and Huang, 2007). Second, although $\beta \beta$-hopanes are the presumed biological isomer, $\beta \alpha$-hopanoids have been identified in some organisms. The compounds $21 \alpha(\mathrm{H})$-moret-22(29)-ene $\left(\mathrm{C}_{30} \mathrm{H}_{50}\right)$ and 30nor-21 $\alpha$-hopan-22-one (isoadiantone; $\mathrm{C}_{29} \mathrm{H}_{48} \mathrm{O}$ ) were isolated from ferns and lichens (Hveding-Bergseth et al., 1983; Shiojima and Ageta, 1990; Tsuzuki et al., 2001). Moretenone and moretenol were isolated from a small shrub (Lavie et al., 1968), and Rosa-Putra et al. (2001) discovered that soil bacteria Frankia spp. synthesize 22(S)moretan-29-ol $\left(\mathrm{C}_{30} \mathrm{H}_{52} \mathrm{OH}\right)$.

Based on the evidence discussed above, some researchers have argued that the PTB moretane anomaly signals increased organic matter input from higher plant material, particularly peat and coal (Wang, 2007; Xie et al., 2007). The data presented in this paper do not support this explanation. None of the previous reports linking higher plant organic matter input to elevated moretane abundances provide a viable explanation for the parallel trends of the extended homohopanes and the $2 \alpha$ - and $3 \beta$ methylhomohopanes because, with the exception of the peat samples reported by Quirk et al. (1984), the $\beta \alpha$-hopanoids detected in organisms, peat, and coal are $\mathrm{C}_{29}$ or $\mathrm{C}_{30}$ compounds. Because thermal maturity and a change in input of higher plant organic matter fail to account for the observed moretane anomalies, other source input changes and diagenetic effects must be considered.

Redox potential is known to affect the distribution of terpanes in lipid extracts (Peters et al., 2005). Multiple redox indicators are presented for the Meishan section in Cao et al., 2009. Values of 28,30-dinorhopane (28,30-DNH) between 0.5 and $2 \%$ and the detection of isorenieratane and $\mathrm{C}_{14-27}$ aryl isoprenoids throughout the cored interval indicates that all of the sediments were deposited under reducing conditions. However, $\mathrm{C}_{35} \mathrm{HHI}$ values are highly variable through the drilled interval $(0.10-14.47 \%)$, which is 
inconsistent with the multiple redox indicators that suggest reducing conditions throughout the cored interval. Homohopane distribution depends on depositional redox conditions, sulfur incorporation in organic matter, and biodegradation (Peters and Moldowan, 1991). The rocks through the entire cored interval were deposited in a marine setting that likely had the necessary sulfate concentrations required for homohopane distributions to reflect redox conditions. The discrepancy of the $\mathrm{C}_{35} \mathrm{HHI}$ with the other redox indicators and the strong relationship of the $\mathrm{C}_{35} \mathrm{HHI}$ with the moretane/hopane ratios, $\mathrm{Ts} /(\mathrm{Ts}+\mathrm{Tm})$, and lithological data suggest a detrital source for the clays and hopanes in the intervals of elevated moretane/hopane ratios.

Multiple lines of evidence suggest increased soil erosion, forest fires, continental weathering, and terrigenous input during the PTB (Ward et al., 2000; Retallack et al., 1998, 2005; Sephton et al., 2005; Wang and Visscher, 2007; Cao et al., 2009; Nabbefeld et al., 2010b; S. Shen et al., 2011; W. Shen et al., 2011). Although most studies do not examine evidence earlier than the Changhsingian, detection of particular polycyclic aromatic hydrocarbons (PAHs) in samples from Meishan prior to the extinction horizon suggests that there may have been earlier pulses of land-derived organic matter to the marine system (Nabbefeld et al., 2010b). Interestingly, the highest values of retene, a PAH thought to be derived from coniferous resin (Peters et al., 2005), is concurrent with the earliest interval of moretane/hopane enrichment in the Lungtan Formation. Accordingly, there may have been increased terrigenous input to the marine system during the PTB and the Wuchiapingian, which is consistent with a detrital origin of clays and hopanes during these intervals.

Increased organic matter input from higher plant matter cannot explain the parallel patterns of $\beta \alpha /(\alpha \beta+\beta \alpha)$ for $C_{30-34}$ homohopanes and $C_{31-33}$ methylhomohopanes, but increased input of hopanoids from soil bacteria may explain the anomalous moretane/hopane ratios observed in all hopane series. The hypothesis that there was an influx of hopanoids from soil bacteria to the marine system is supported by the 
observation that high moretane/hopane ratios for all hopane series are associated with high hopane/sterane percentages. This hypothesis is further supported by the observation that high hopane/sterane percentages are associated with high percentages of total clay and high abundances of the clay types that are most tightly related to moretane/hopane ratios (chlorite, illite, mixed layer illite/smectite, and smectite). We conclude that the multiple intervals of elevated moretanes were characterized by increased input of hopanoids derived from soils. However, this change in source input alone does not fully explain the moretane stereochemical anomalies. While $\beta \alpha$ hopanoids have been identified in one type of soil bacteria (Rosa-Putra et al. 2001), it is more likely that the detrital clays and redox conditions of the original depositional environment controlled the stereochemistry of the soil-derived hopanoids that were ultimately deposited in marine sediments. Therefore, a combination of hopanoid input from soils and diagenetic effects generated the unique moretane/hopanes patterns observed at Meishan.

\subsubsection{Discussion of Possible Mechanisms for Mineral Preservation of $\beta \alpha$-hopanoid Stereochemistry}

Certain minerals are known to mediate diagenetic reactions. In addition to thermal maturity and the source of organic matter, depositional environment and lithology are known to affect the distribution of biomarkers, including the moretane/hopane ratio (Moldowan et al., 1986; Curiale and Odermatt, 1989; Peters et al., 2005). If given sufficient time and heat, the biological isomers decline relative to the thermodynamically preferred isomers. However, this simplistic view is further complicated by factors that affect the relative rates of hydrocarbon generation, isomerization, and thermal degradation (Lu et al., 1989; Farrimond et al., 1998). Mineral composition has been shown to affect the degree of isomerization for steranes and hopanes in natural systems as well as laboratory pyrolysis experiments (Rullkötter et 
al., 1985; Eglinton et al., 1986; Curiale and Odermatt, 1989; Lu et al., 1989; Peters et al., 1990; Farrimond et al., 1998; Pan et al., 2010), but distinguishing between mineral effects on biomarker genesis, isomerization, and thermal degradation is difficult. Moreover, it is possible that a combination of these mineral composition effects plays a role in controlling biomarker stereoisomer distribution.

Originally, it was believed that the biologically preferred $\beta \beta$-hopane was converted to the $\beta \alpha$-hopane and $\alpha \beta$-hopane according to the energy diagram and schematic depicted in Seifert and Moldowan (1980). The thermodynamic stabilities of the hopane stereoisomers were later confirmed by molecular mechanics (Kolaczkowska et al., 1990). However, it was recognized that minerals could modulate the rates of epimerization. In particular, acidic surface sites of certain clay types were shown to catalyze isomerization, rearrangement, and hydrogen exchange reactions (Solomon and Swift, 1967; Sieskind et al., 1979; Saxby et al., 1992). Alexander et al. (1984) proposed an epimerization mechanism that proceeded through a planar $\mathrm{sp}^{2}$ hybridized intermediate. Due to the enhanced stabilities of tertiary, allylic, or benzylic carbocation and radical intermediates, these positions interact with clay surface sites. The carbon adjacent to the carbocation or radical intermediate then undergoes hydrogen exchange and loss of the original stereochemistry if the hydrogen is added from the opposite face (Alexander et al., 1984). If the mechanism proposed by Alexander et al. (1984) is correct, claycatalyzed epimerization may be stereoselective. In which case, the conversion of $\beta \beta$ hopane to $\alpha \beta$-hopane would be less favorable than the conversion to $\beta \alpha$-hopane because $\mathrm{C}_{22}$ is arguably the most accessible tertiary carbon in the hopane skeleton that could interact with the clay surface and form a carbocation intermediate, thus promoting epimerization at the adjacent C-21.

However, direct epimerization of the free $\beta \beta$-hopane to $\beta \alpha$-hopane and $\alpha \beta$ hopane isomers is not the only factor that affects the isomeric distribution of hopanes. Indeed, the rates of hydrocarbon release from kerogen and asphaltene, rates of 
generation of hydrocarbons from functionalized moieties, and rates of hydrocarbon thermal degradation may also play significant roles in controlling isomer distributions of biomarkers. Mineralogy may mediate these processes, thus influencing the initial and final biomarker distribution (Eglinton et al., 1986; Huizinga et al., 1987; Larcher et al., 1988; Lu et al., 1989; Abbott et al., 1990; Bishop and Abbott, 1993; Bishop et al., 1998; Farrimond et al., 1998, 2002; Koopmans et al., 1998; Wei et al., 2006; Pan et al., 2009, 2010). Furthermore, both organic and inorganic protective matrices can affect lipid biomarker distributions (Hedges and Keil, 1995; Huang et al., 2008; Mead and Goni, 2008). Active clay surfaces, in particular, tend to selectively adsorb polar compounds (Pan et al., 2005). For this reason, the preservation and generation mechanisms of polar hopane precursor compounds may offer insight to the moretane anomalies at Meishan detailed in this report.

Hopanoic acids can be a significant source of free hopanes (Bennett and Abbott, 1999). Laboratory and field results have demonstrated that the degree of isomerization of free hopanes is greater than kerogen-bound and functionalized hopanoids, including hopanoic acids (Tannenbaum et al., 1986; Peters and Moldowan, 1991; Bishop et al., 1998; Murray et al., 1998; Farrimond et al., 2002; Lockhart et al., 2008). Isomerization in the hopane E-ring may occur during the decarboxylation of hopanoic acids to yield free hopanes or during bond cleavage of kerogen-bound hopanoids (Farrimond et al., 1998, 2002). Alternatively, rates of isomerization may be slower for bound hopanoids and functionalized hopanoids than for free hopanes, as discussed below.

Hopanoic acids released from kerogen or produced during early diagenesis may become adsorbed on the mineral matrix and/or polar organic matter by ionic interactions (Huizinga et al., 1987; Thomas et al., 1993; Kubicki et al., 1999). Previous works show that "trapped" or "bound" hydrocarbons isomerize at slower rates than free hydrocarbons (Derenne et al., 1988; Jaffé and Gardinali, 1990; Jaffé et al., 1997). Likewise, adsorption of polar moretane precursors to the surface of a protective matrix 
could effectively retard the rates of isomerization until release at high maturities. Carboxylic acids tend to adsorb strongly on inorganic surfaces (Thomas et al., 1993). However, the preservation potential of the less mature hopanoic acid signature depends on the adsorptive capacity of the mineral surface (Ransom et al., 1998; Kubicki et al., 1999). The different surface chemistries associated with individual clay types have unique affinities for organic matter, which could explain the different correlation strengths observed for $\beta \alpha /(\alpha \beta+\beta \alpha)$ and the different clay types at Meishan. However, a more comprehensive study is required that characterizes the interaction of polar precursors of hopanoids with the clay types at Meishan and monitors hopanoic acid interactions with the mineral surface over a range of temperature and times.

\subsubsection{Discussion of Depositional Environment, Source Input, and Ts/(Ts+Tm)}

Like the moretane/hopane ratios, the $\mathrm{Ts} /(\mathrm{Ts}+\mathrm{Tm})$ thermal maturity parameter is also strongly influenced by source input, lithology, oxicity, and acidity of depositional environment (McKirdy et al., 1983; Moldowan et al., 1986; Waples and Machihara, 1990; Dahl et al., 1993; Peters et al., 2005; Bennett and Olsen, 2007). According to molecular mechanics and observation, $\operatorname{Tm}$ (or $\mathrm{C}_{27} 17 \alpha$-trisnorhopane) is less stable than the rearranged $\mathrm{Ts}$ isomer (or $\mathrm{C}_{27}$ 18 $\alpha$-trisnorhopane) (Seifert and Moldowan, 1978; Kolaczkowska et al., 1990). According to Fig. 6, Ts/(Ts+Tm) seems to be related not only to $C_{30} \beta \alpha /(\alpha \beta+\beta \alpha)$ as shown in Cao et al. (2009), but it is also inversely related to the $\beta \alpha /(\alpha \beta+\beta \alpha)$ values of the homohopane and $2 \alpha-$ and $3 \beta$ - methylhomohopane series. This inverse relationship would be expected for $\mathrm{Ts} /(\mathrm{Ts}+\mathrm{Tm})$ and $\beta \alpha /(\alpha \beta+\beta \alpha)$ if thermal maturity was the primary control on these parameters. Like $\beta \alpha /(\alpha \beta+\beta \alpha)$, Ts/(Ts+Tm) covaries with total clay percent, specific clay type abundances, $\mathrm{C}_{35} \mathrm{HHI}$, and hopane/sterane percentages at the Meishan section. As discussed above, maturity parameters that more appropriately evaluate the thermal history of this section, indicate relatively constant thermal maturity through the section, so the same mechanisms that 
are influencing the moretane/hopane ratios are likely affecting $\mathrm{Ts} /(\mathrm{Ts}+\mathrm{Tm})$. Unlike previous studies that show that carbonate source rocks tend to generate oil with lower Ts/(Ts+Tm) values than clay-rich source rocks (McKirdy et al., 1983; Waples and Machihara, 1990; Peters et al., 2005; Bennett and Olsen, 2007), our results show that low values of $\mathrm{Ts} /(\mathrm{Ts}+\mathrm{Tm})$ coincide with intervals of high clay content. However, our results are consistent with previous work that indicates $\mathrm{Tm}$ is favored over the rearranged, more stable isomer Ts in oxidizing conditions (Moldowan et al., 1986). Like our results that show high values of $\mathrm{Ts} /(\mathrm{Ts}+\mathrm{Tm})$ in carbonate-rich rocks deposited under reducing conditions, Rullkötter and Marzi, (1988) found higher values of Ts/(Ts+Tm) associated with the relatively carbonate-rich Lias $\varepsilon$ unit which was deposited under reducing conditions compared to the adjacent carbonate-poor mudstone facies that was deposited under oxic conditions. Input of soil-derived hopanoids seems to play a role in the moretane/hopane ratio patterns, and the relationship of $\mathrm{Ts} /(\mathrm{Ts}+\mathrm{Tm})$ with hopane/sterane percentages suggests that source input may be affecting $\mathrm{Ts} /(\mathrm{Ts}+\mathrm{Tm})$ as well. In total, these results indicate that a combination of source input effects and depositional factors, including oxicity and lithology, control Ts/(Ts+Tm) and complicate the interpretation of this geochemical indicator.

\subsubsection{Discussion of Lithology, $\mathrm{C}_{35}$ Homohopane Index and Additional Molecular Parameters}

Lithology and redox conditions affect other molecular maturity parameters in addition to moretane/hopane ratios and Ts/(Ts+Tm) (Peters et al., 2005). Molecular parameters from Cao et al. (2009), including 28,30-bisnorhopane/C 30 hopane, gammacerane/ $\mathrm{C}_{30}$ hopane, $\mathrm{C}_{29}$ diasterane/regular sterane, and $\mathrm{C}_{27}$ diasterane/regular

sterane were plotted against percent carbonate, percent total clay, percent total clay/TOC, $\mathrm{C}_{35} \mathrm{HHI}$, and hopane/sterane percentages. No significant correlations were identified with the exception of an inverse correlation of $\mathrm{C}_{29}$ diasterane/regular sterane 
and $\mathrm{C}_{27}$ diasterane/regular sterane with hopane/sterane percentages. The absence of any relationship with clay/TOC is counter to the results of van Kaam-Peters et al. (1998) and Nabbefeld et al. (2010a), although the different environmental settings, lithologies, and methods may partially explain the discrepancy.

Having recognized a detrital, clay-borne contribution to the hopanoid inventory during intervals of enhanced moretane accumulation at Meishan, the absence of a relationship between diasterane/regular sterane ratio and lithology comes into focus. Terrigenous organic matter tends to have higher abundances of hopanoids and lower contents of steroids relative to TOC compared to marine organic matter (Peters et al., 2005; Handley et al., 2010; Sáenz et al., 2011). Thus, in marine settings the sources of steroids are mainly marine algae from the water column. During sedimentation in a strongly reducing setting, steroids may be relatively unaffected by diagenetic processes associated with the clays. In other words, in this particular environment steroid and hopanoid diagenetic processes are decoupled to the degree that some fraction of the hopanoids is derived from detrital sources while the sterols are predominantly of local origin.

\subsubsection{Potential Sources of Berthierine}

The discovery of measurable berthierine and evidence of trace berthierine in the Lungtan and Yinkeng Formations, respectively, is an observation with multiple possible environmental implications. Berthierine forms in reducing environments, but low levels of sulfide and bicarbonate are also required because pyrite or siderite formation is favored over berthierine formation in reducing conditions in the presence of sulfide and bicarbonate, respectively (Taylor and Curtis; 1995; Fritz and Toth, 1997; Sheldon and Retallack, 2002). Interestingly, the berthierine-rich samples occur in the only kaoliniterich interval of the section, and kaolinite is implicated in the formation of berthierine (Sheldon and Retallack, 2002). In contrast, samples from the Yinkeng Formation with 
evidence of trace berthierine are kaolinite-poor and smectite-rich. Smectite and kaolinite signify different weathering conditions, and as a result, the clay composition of these two intervals indicates that two different soil types are contributing to the sediments.

The detection of berthierine in samples from the Lungtan Formation where low $\mathrm{C}_{35} \mathrm{HHI}$ values indicates oxic conditions suggests that one or both of these redox indicators have a detrital component. While it is not possible to conclusively determine from our results whether the berthierine is authigenic or detrital, it is interesting to note that berthierine was discovered in high latitude paleosols deposited in the Early Triassic following the PTB (Sheldon and Retallack, 2002). Formation of berthierine in soils is rare because of the environmental requirements for formation, but weathering or erosion of berthierine-containing paleosols could contribute detrital berthierine to the marine setting. The berthierine-containing paleosols reported in Sheldon and Retallack, 2002 do not correspond well spatially or temporally with the berthierine-rich samples in the Lungtan Formation, but the discovery of berthierine in paleosols may offer a possible mechanism for delivery of berthierine to marine sediments in the event that the berthierine is detrital.

\subsection{CONCLUSIONS}

Three periods of moretane enhancement, two in the Triassic and one in the Permian, are recorded in the core from the Meishan section in southeastern China. These $C_{30}$ moretane/hopane excursions are echoed in the homohopanes and $2 \alpha$ - and $3 \beta$ methylhomohopanes. In light of the multiple and parallel moretane 'anomalies' for the homohopane and $2 \alpha$ - and $3 \beta$-methylhomohopane series, enhanced input from higher plant organic matter does not adequately explain the observed hopane isomer patterns at Meishan. Correlation of high moretane/hopane ratios with low $\mathrm{C}_{35} \mathrm{HHI}$ and high hopane/sterane ratios suggest increased input of hopanoids from soils that are 
influenced by oxic conditions during transport. However, this shift in source input alone cannot explain the hopane stereochemical patterns at Meishan. Based on $\mathrm{C}_{35} \mathrm{HHI}$ and mineralogical analyses, we conclude that diagenetic processes related to lithology and redox determine the $\beta \alpha$-moretane/ $\alpha \beta$-hopane distribution for all of the hopane series throughout the cored interval. It seems likely that certain clay types preferentially bind triterpanes having the moretane configuration. Berthierine, a clay that is formed in reducing conditions, was detected in samples from the Lungtan Formation. It is unclear from our results whether the berthierine is authigenic of detrital, but future determination of the origin of berthierine at Meishan may offer additional environmental insight.

Additional molecular parameters were tested for relationships with lithology. Hopane/sterane ratios, $\mathrm{C}_{35} \mathrm{HHI}$, percent total clay, chlorite, illite, and illite/smectite mixed layer clay showed significant relationships with $\mathrm{Ts} /(\mathrm{Ts}+\mathrm{Tm})$, suggesting source input, lithology, and redox also affect this ratio. However, unlike previous studies (van Kaam-Peters et al., 1998; Nabbefeld et al., 2010a) that reported a link between diasteranes and percent clay/TOC, this relationship was noticeably absent in the sediments at Meishan. Therefore, it seems likely that the diagenesis of steroids here was decoupled from the clay component and more under the control of the intense reducing conditions that prevailed in the water column. In total, our results point toward a complex role of redox and lithology in the transformation of organic matter during diagenesis and maturation. Future work is required to disentangle the lithological effects on diagenetic processes, including biomarker genesis isomerization, and thermal degradation. We conclude that a combination of episodic hopanoid input from soil bacteria and diagenetic effects related to redox and detrital clays generated the distinctive moretane/hopane patterns at Meishan. 


\subsection{Acknowledgments}

We wish to thank Christian Hallman and Jürgen Rullkötter for discussions and constructive suggestions, and Bob Burruss, Simon George, and Ken Peters for informative reviews and insightful comments that strengthened this work. KLF is supported by a National Science Foundation Graduate Fellowship. Research at MIT was supported by an award (NNX09AM88G) from the NASA Exobiology Program to RES. Research at Nanjing was supported by the 973 Project of the MST of China (2011CB808905) and NSF of China. NJT acknowledges support from Churchill College and the Royal Society (RG 2009/R2).

\subsection{References}

Abbott G., Wang G., Eglinton T., Home A., Petch G., 1990. The kinetics of sterane biological marker release and degradation processes during the hydrous pyrolysis of vitrinite kerogen. Geochim. Cosmochim. Acta 54, 2451-2461.

Aichner B., Wilkes H., Herzschuh U., Mischke S., Zhang C., 2010. Biomarker and compoundspecific $\delta^{13} \mathrm{C}$ evidence for changing environmental conditions and carbon limitation at Lake Koucha, eastern Tibetan Plateau. J. Paleolimnol. 43, 873-899.

Alexander R., Kagi R., Larcher A., 1984. Clay catalysis of alkyl hydrogen exchange reactions-reaction mechanisms. Org. Geochem. 6, 755-760.

Barnosky A.D., Matzke N., Tomiya S., Wogan G.O.U., Swartz B., Quental T.B., Marshall C., Mcguire J.L., Lindsey E.L., Maguire K.C., Mersey B., Ferrer E.A., 2011. Has the Earth's sixth mass extinction already arrived? Nature 470, 51-57.

Bauer P., Dunlap N., Arseniyadis S., Watt D., Seifert W., Moldowan J., 1983. Synthesis of biological markers in fossil fuels. $1.17 \alpha$ and $17 \beta$ isomers of 30-norhopane and 30normoretane. J. Org. Chem. 48, 4493-4497. 
Becker L., Poreda R., Hunt A., Bunch T., Rampino M., 2001. Impact event at the PermianTriassic boundary: evidence from extraterrestrial noble gases in fullerenes. Science 291, 1530-1533.

Bennett B., Abbott G., 1999. A natural pyrolysis experiment - hopanes from hopanoic acids? Org. Geochem. 30, 1509-1516.

Bennett B., Olsen S.D., 2007. The influence of source depositional conditions on the hydrocarbon and nitrogen compounds in petroleum from central Montana, USA. Org. Geochem. 38, 935-956.

Benton M., Twitchett R., 2003. How to kill (almost) all life: the end-Permian extinction event. Trends Ecol. Evol. 18, 358-365.

Bishop A., Abbott G., 1993. The interrelationship of biological marker maturity parameters and molecular yields during contact-metamorphism. Geochim. Cosmochim. Acta 57, 3661-3668.

Bishop A., Love G., McAulay A., Snape C., Farrimond P., 1998. Release of kerogen-bound hopanoids by hydropyrolysis. Org. Geochem. 29, 989-1001.

Campbell I., Czamanske G., Fedorenko V., Hill R., Stepanov V., 1992. Synchronism of the Siberian Traps and the Permian-Triassic boundary. Science 258, 1760-1763.

Cao C., Love G.D., Hays L., Wang W., Shen S., Summons R.E., 2009. Biogeochemical evidence for euxinic oceans and ecological disturbance presaging the end-Permian mass extinction event. Earth. Planet. Sci. Lett. 281, 188-201.

Cao C., Wang W., Jin Y., 2002. Carbon isotope excursions across the Permian-Triassic boundary in the Meishan section, Zhejiang Province, China. Chinese Sci. Bull. 47, 1125-1129.

Curiale J., Odermatt J., 1989. Short-term biomarker variability in the Monterey Formation, Santa Maria Basin. Org. Geochem. 14, 1-13.

Dahl J., Michael Moldowan J., Sundararaman P., 1993. Relationship of biomarker distribution to depositional environment: Phosphoria Formation, Montana, USA. Org. Geochem. 20, 1001-1017.

Derenne S., Largeau C., Casadevall E., Connan J., 1988. Comparison of torbanites of various origins and evolutionary stages. Bacterial contribution to their formation. Causes of the lack of botryococcane in bitumens. Org. Geochem. 12, 43-59. 
Eglinton T., Rowland S., Curtis C., Douglas A., 1986. Kerogen-mineral reactions at raised temperatures in the presence of water. Org. Geochem. 10, 1041-1052.

Erwin D.H., 2006. Extinction: how life on earth nearly ended 250 million years ago. Princeton University Press.

Farrimond P., Griffiths T., Evdokiadis E., 2002. Hopanoic acids in Mesozoic sedimentary rocks: their origin and relationship with hopanes. Org. Geochem. 33, 965-977.

Farrimond P., Taylor A., Telnaes N., 1998. Biomarker maturity parameters: the role of generation and thermal degradation. Org. Geochem. 29, 1181-1197.

Fritz S., Toth T., 1997. An Fe-berthierine from a Cretaceous laterite. 2. Estimation of Eh, $\mathrm{pH}$ and $\mathrm{pCO}_{2}$ conditions of formation. Clays Clay Miner. 45, 580-586.

Grantham P., 1986. Sterane isomerisation and moretane/hopane ratios in crude oils derived from Tertiary source rocks. Org. Geochem. 9, 293-304.

Grice K., Cao C., Love G., Bottcher M., Twitchett R., Grosjean E., Summons R., Turgeon S., Dunning W., Jin Y., 2005. Photic zone euxinia during the Permian-Triassic superanoxic event. Science 307, 706-709.

Handley, L., Talbot, H., Cooke, M., Anderson, K., Wagner, T. 2010. Bacteriohopanepolyols as tracers for continental and marine organic matter supply and phases of enhanced nitrogen cycling on the late Quaternary Congo deep sea fan. Org. Geochem. 41, 910-914.

Hedges J.I., Keil R.G., 1995. Sedimentary organic matter preservation: an assessment and speculative synthesis. Mar. Chem. 49, 81-115.

Huang X., Cui J., Pu Y., Huang J., Blyth A.J., 2008. Identifying "free" and "bound" lipid fractions in stalagmite samples: An example from Heshang Cave, Southern China. Appl. Geochem. 23, 2589-2595.

Hughes W.B., Dzou L.I.P., 1995. Reservoir overprinting of crude oils. Org. Geochem. 23, 905-914.

Huizinga B., Tannenbaum E., Kaplan I., 1987. The role of minerals in the thermal alteration of organic-matter. 4. Generation of normal-alkanes, acyclic isoprenoids, and alkenes in laboratory experiments. Geochim. Cosmochim. Acta 51, 1083-1097.

Hveding-Bergseth N., Bruun T., Kjųsen H., 1983. Isolation of 30-nor-21 [alpha]-hopan-22-one (isoadiantone) from the lichen Platismatia glauca. Phytochemistry 22, 1826-1827. 
Isaksen G., Bohacs K., 1995. Geologic controls of source rock geochemistry through relative sea level, in: Katz, B. (Ed.), Petroleum Source Rocks. Springer-Verlag, Berlin; New York, pp. 25-50.

Ishiwatari R., Yamamoto S., Uemura H., 2005. Lipid and lignin/cutin compounds in Lake Baikal sediments over the last $37 \mathrm{kyr}$ : implications for glacial-interglacial palaeoenvironmental change. Org. Geochem. 36, 327-347.

Jaffé R., Diaz D., Hajje N., Chen L., Eckardt C., Furton K.G., 1997. Hydrocarbon speciation in ancient sediments studied by stepwise high-temperature supercritical carbon dioxide extraction. Org. Geochem. 26, 59-65.

Jaffé R., Gardinali P., 1990. Generation and maturation of carboxylic acids in ancient sediments from the Maracaibo Basin, Venezuela. Org. Geochem. 16, 211-218.

Kaiho K., Chen Z.-Q., Kawahata H., Kajiwara Y., Sato H., 2006. Close-up of the end-Permian mass extinction horizon recorded in the Meishan section, South China: Sedimentary, elemental, and biotic characterization and a negative shift of sulfate sulfur isotope ratio. Palaeogeogr., Palaeoclimatol., Palaeoecol. 239, 396-405.

Kaiho K., Kajiwara Y., Nakano T., Miura Y., Kawahata H., Tazaki K., Ueshima M., Chen Z., Shi G., 2001. End-Permian catastrophe by a bolide impact: Evidence of a gigantic release of sulfur from the mantle. Geology 29, 815-818.

Kamo S., Czamanske G., Amelin Y., Fedorenko V., Davis D., Trofimov V., 2003. Rapid eruption of Siberian flood-volcanic rocks and evidence for coincidence with the Permian-Triassic boundary and mass extinction at $251 \mathrm{Ma}$. Earth. Planet. Sci. Lett. 214, 75-91.

Kolaczkowska E., Slougui N., Watt D., Maruca R., Michael Moldowan J., 1990. Thermodynamic stability of various alkylated, dealkylated and rearranged $17 \alpha$-and $17 \beta$-hopane isomers using molecular mechanics calculations. Org. Geochem. 16, 1033-1038.

Koopmans M., Carson F., Sinninghe Damsté J., Lewan M., 1998. Biomarker generation from Type II-S kerogens in claystone and limestone during hydrous and anhydrous pyrolysis. Org. Geochem. 29, 1395-1402.

Kristen I., Wilkes H., Vieth A., Zink K.-G., Plessen B., Thorpe J., Partridge T.C., Oberhaensli H., 2010. Biomarker and stable carbon isotope analyses of sedimentary organic matter from Lake Tswaing: evidence for deglacial wetness and early Holocene drought from South Africa. J Paleolimnol 44, 143-160. 
Kubicki J., Schroeter L., Itoh M., Nguyen B., Apitz S., 1999. Attenuated total reflectance Fouriertransform infrared spectroscopy of carboxylic acids adsorbed onto mineral surfaces. Geochim. Cosmochim. Acta 63, 2709-2725.

Larcher A., Alexander R., Kagi R., 1987. Changes in configuration of extended moretanes with increasing sediment maturity. Org. Geochem. 11, 59-63.

Larcher A., Alexander R., Kagi R., 1988. Differences in reactivities of sedimentary hopane diastereomers when heated in the presence of clays. Org. Geochem. 13, 665-669.

Lavie D., Jain T., Mahendra K., 1968. Terpenoids--VII. Constituents of Euphorbia lateriflora Schum. and Thonn. Phytochemistry 7, 657-660.

Lockhart R.S., Meredith W., Love G.D., Snape C.E., 2008. Release of bound aliphatic biomarkers via hydropyrolysis from Type II kerogen at high maturity. Org. Geochem. 39, 1119-1124.

Looy C., Twitchett R., Dilcher D., Konijnenburg-Van Cittert J., Visscher H., 2001. Life in the endPermian dead zone. Proc. Natl. Acad. Sci. U. S. A. 98, 7879-7883.

Lu S., Ruth E., Kaplan I., 1989. Pyrolysis of kerogens in the absence and presence of montmorillonite- I. The generation, degradation and isomerization of steranes and triterpanes at 200-Degrees-C and 300-Degrees-C. Org. Geochem. 14, 491-499.

McKirdy D., Aldridge A., Ypma P., 1983. A geochemical comparison of some crude oils from Pre-Ordivician carbonate rocks, in: Bjoroy, M. (Ed.), Advances in Organic Geochemistry. John Wiley and Sons, New York, pp. 99-107.

Mead R.N., Goni M.A., 2008. Matrix protected organic matter in a river dominated margin: A possible mechanism to sequester terrestrial organic matter? Geochim. Cosmochim. Acta 72, 2673-2686.

Metcalfe I., Isozaki Y., 2009. Current perspectives on the Permian-Triassic boundary and endPermian mass extinction: Preface. J. Asian Earth Sci. 36, 407-412.

Michaelsen P., 2002. Mass extinction of peat-forming plants and the effect on fluvial styles across the Permian-Triassic boundary, northern Bowen Basin, Australia. Palaeogeogr., Palaeoclimatol., Palaeoecol. 179, 173-188.

Moldowan J., Sundararaman P., Schoell M., 1986. Sensitivity of biomarker properties to depositional environment and/or source input in the Lower Toarcian of SW-Germany. Org. Geochem. 10, 915-926. 
Mundil R., Ludwig K., Metcalfe I., Renne P., 2004. Age and timing of the Permian mass extinctions: $\mathrm{U} / \mathrm{Pb}$ dating of closed-system zircons. Science 305, 1760-1763.

Mundil R., Metcalfe I., Ludwig K.R., Renne P.R., Oberli F., Nicoll R.S., 2001. Timing of the Permian-Triassic biotic crisis: implications from new zircon $\mathrm{U} / \mathrm{Pb}$ age data (and their limitations). Earth. Planet. Sci. Lett. 187, 131-145.

Murray I., Love G., Snape C., Bailey N., 1998. Comparison of covalently-bound aliphatic biomarkers released via hydropyrolysis with their solvent-extractable counterparts for a suite of Kimmeridge clays. Org. Geochem. 29, 1487-1505.

Nabbefeld B., Grice K., Schimmelmann A., Summons R.E., Troitzsch U., Twitchett R.J., 2010a. A comparison of thermal maturity parameters between freely extracted hydrocarbons (Bitumen I) and a second extract (Bitumen II) from within the kerogen matrix of Permian and Triassic sedimentary rocks. Org. Geochem. 41, 78-87.

Nabbefeld, B., Grice, K., Summons, R.E., Hays, L.E., Cao, C., 2010b. Significance of polycyclic aromatic hydrocarbons (PAHs) in Permian/Triassic boundary sections. Appl. Geochem. $25,1374-1382$.

Ourisson G., Albrecht P., 1992. Hopanoids. 1. Geohopanoids - The most abundant naturalproducts on earth. Acc. Chem. Res. 25, 398-402.

Pan C., Feng J., Tian Y., Yu L., Luo X., Sheng G., Fu J., 2005. Interaction of oil components and clay minerals in reservoir sandstones. Org. Geochem. 36, 633-654.

Pan C., Geng A., Zhong N., Liu J., 2010. Kerogen pyrolysis in the presence and absence of water and minerals: steranes and triterpenoids. Fuel 89, 336-345.

Pan C., Geng A., Zhong N., Liu J., Yu L., 2009. Kerogen pyrolysis in the presence and absence of water and minerals: Amounts and compositions of bitumen and liquid hydrocarbons. Fuel 88, 909-919.

Peters K., Moldowan J., 1991. Effects of source, thermal maturity, and biodegradation on the distribution and isomerization of homohopanes in petroleum. Org. Geochem. 17, 47-61.

Peters K., Moldowan J., Sundararaman P., 1990. Effects of hydrous pyrolysis on biomarker thermal maturity parameters: Monterey phosphatic and siliceous members. Org. Geochem. 15, 249-265. 
Peters K.E., Walters C.C., Moldowan J.M., 2005. The biomarker guide, 2nd ed. Cambridge University Press, Cambridge, UK ; New York.

Pollastro R.M., 1993. Considerations and applications of the illite/smectite geothermometer in hydrocarbon-bearing rocks of Miocene to Mississippian age. Clays Clay Miner. 41, 119133.

Quirk M., Wardroper A., Wheatley R., Maxwell J., 1984. Extended hopanoids in peat environments. Chem. Geol. 42, 25-43.

Ransom B., Kim D., Kastner M., Wainwright S., 1998. Organic matter preservation on continental slopes: importance of mineralogy and surface area. Geochim. Cosmochim. Acta 62, 1329-1345.

Raup D., 1979. Size of the Permo-Triassic bottleneck and its evolutionary implications. Science 206, 217-218.

Renne P., Black M., Zichao Z., Richards M., Basu A., 1995. Synchrony and causal relations between Permian-Triassic boundary crises and Siberian flood volcanism. Science 269, 1413-1416.

Retallack G., 1995. Permian-Triassic life crisis on land. Science 267, 77-80.

Retallack G., Seyedolali A., Krull E., Holser W., Ambers C., Kyte F., 1998. Search for evidence of impact at the Permian-Triassic boundary in Antarctica and Australia. Geology 26, 979982.

Retallack, G., 2005. Earliest Triassic claystone breccias and soil-erosion crisis. Journal of Sedimentary Research. 75. 679-695.

Reynolds Jr. R., Reynolds R., 1996. NEWMOD for windows. The calculations of one dimensional x-ray diffraction patterns of mixed-layered clay minerals, Hanover, NH.

Rosa-Putra S., Nalin R., Domenach A., Rohmer M., 2001. Novel hopanoids from Frankia spp. and related soil bacteria. Eur. J. Biochem. 268, 4300-4306.

Rullkötter J., Marzi R., 1988. Natural and artificial maturation of biological markers in a Toarcian shale from northern Germany. Org. Geochem. 13, 639-645. 
Rullkötter J., Spiro B., Nissenbaum A., 1985. Biological marker characteristics of oils and asphalts from carbonate source rocks in a rapidly subsiding graben, Dead Sea, Israel. Geochim. Cosmochim. Acta 49, 1357-1370.

Sáenz, J., Eglinton, T., Summons, R., 2011. Abundance and structural diversity of bacteriohopanepolyols in suspended particulate matter along a river ocean transect. Org. Geochem.42, 774-780.

Saxby J., Chatfield P., Taylor G., Fitzgerald J., Kaplan I., Lu S., 1992. Effect of clay minerals on products from coal maturation. Org. Geochem. 18, 373-383.

Seifert W., Moldowan J., 1980. The effect of thermal stress on source-rock quality as measured by hopane stereochemistry. Phys Chem Earth 12, 229-237.

Seifert W., Moldowan M., 1978. Applications of steranes, terpanes and monoaromatics to the maturation, migration and source of crude oils. Geochim. Cosmochim. Acta 42, 77-95.

Sephton M.A., Looy C.V., Brinkhuis H., Wignall P.B., De Leeuw J.W., Visscher H., 2005. Catastrophic soil erosion during the end-Permian biotic crisis. Geology 33, 941-944.

Sepkoski J.J., 2002. A compendium of fossil marine animal genera. Bull. Am. Paleontol. 363. 1-563.

Sheldon, N. D., Retallack, G. J., 2002. Low oxygen levels in earliest Triassic. Geology. 30. 919-922.

Shen J., Huang W., 2007. Biomarker distributions as maturity indicators in coals, coaly shales, and shales from Taiwan. Terr Atmos Ocean Sci 18, 739-755.

Shen S., Crowley, J.,,Wang, Y., Bowring S., Erwin, D., Sadler, P., Cao C., Rothman, D., Henderson, C., Ramezani, J., Zhang, H., Shen, Y., Wang, X., Wang, W., Mu, L, Li, W., Tang, Y., Liu, X., Liu, L., Zeng, Y., Jiang, Y., Jin, Y., 2011. Calibrating the End-Permian Mass Extinction. Science, 17 November 2011 (10.1126/science.1213454).

Shen, W., Sun, Y., Lin, Y., Liu, D., Chai, P., 2011. Evidence of wildfire in the Meishan section and implications for Permina-Triassic events. Geochim. Cosmochim. Acta 75. 1992-2006.

Shiojima K., Ageta H., 1990. Fern constituents: two new triterpenoid hydrocarbons, hop-16-ene and isohop-22(29)-ene, isolated from Davallia-mariiesii. Chem Pharm Bull 38, 347-349.

Sieskind O., Joly G., Albrecht P., 1979. Simulation of the geochemical transformations of sterols superacid effect of clay-minerals. Geochim. Cosmochim. Acta 43, 1675-1679. 
Solomon D., Swift J., 1967. Reactions catalyzed by minerals. Part II. chain termination in freeradical polymerizations. J. Appl. Polym. Sci. 11, 2567-2575.

Srodon J., Drits V., McCarty D., Hsieh J., Eberl D., 2001. Quantitative X-ray diffraction analysis of clay-bearing rocks from random preparations. Clays Clay Miner. 49, 514-528.

Tannenbaum E., Ruth E., Kaplan I., 1986. Steranes and triterpanes generated from kerogen pyrolysis in the absence and presence of minerals. Geochim. Cosmochim. Acta 50, 805-812.

Taylor K., Curtis C., 1995. Stability and facies association of early diagenetic mineral assemblages; an example from a Jurassic ironstone-mudstone succession, UK. J. Sediment Res. A65, 358-368.

Thomas M., Clouse J., Longo J., 1993. Adsorption of organic compounds on carbonate minerals. 1. Model compounds and their influence on mineral wettability. Chem. Geol. 109, 201213.

Tsuzuki K., Ohashi A., Arai Y., Masuda K., Takano A., Shiojima K., Ageta H., Cai S., 2001. Triterpenoids from Adiantum caudatum. Phytochemistry 58, 363-367.

Twitchett R., Looy C., Morante R., Visscher H., Wignall P., 2001. Rapid and synchronous collapse of marine and terrestrial ecosystems during the end-Permian biotic crisis. Geology 29, 351-354.

Uemura H., Ishiwatari R., 1995. Identification of unusual 17 [beta](H)-moret-22 (29)-ene in lake sediments. Org. Geochem. 23, 675-680.

van Kaam-Peters H.M.E., Köster J., van der Gaast S.J., Dekker M., de Leeuw J.W., Sinninghe Damsté J.S., 1998. The effect of clay minerals on diasterane/sterane ratios. Geochim. Cosmochim. Acta 62, 2923-2929.

Wang C., 2007. Anomalous hopane distributions at the Permian-Triassic boundary, Meishan, China-Evidence for the end-Permian marine ecosystem collapse. Org. Geochem. 38, 52-66.

Wang, C., Visscher, H., 2007. Abundance anomalies of aromatic biomarkers in the PermianTriassic boundary section at Meishan, China - Evidence of end-Permian terrestrial ecosystem collapse.Palaeogeogr. Palaeoclimatol., Palaeoecol. 252, 291-303.

Wang C., Liu Y., Liu H., Zhu L., Shi Q., 2005. Geochemical significance of the relative enrichment of pristane and the negative excursion of delta C-13(Pr) across the PermianMriassic Boundary at Meishan, China. Chinese Sci Bull 50, 2213-2225. 
Waples D., Machihara T., 1990. Applications of sterane and triterpane biomarkers in petroleum exploration. Bulletin Canadian Petroleum Geology 38, 357-380.

Ward, P.D., Montgomery, D.R., Smith, R., 2000. Altered river morphology in South Africa related to the Permian-Triassic Extinction. 289, 1740-1743.

Wei Z., Moldowan J., Paytan A., 2006. Diamondoids and molecular biomarkers generated from modern sediments in the absence and presence of minerals during hydrous pyrolysis. Org. Geochem. 37, 891-911.

Wignall P., Hallam A., 1992. Anoxia as a cause of the Permian/Triassic mass extinction: facies evidence from northern Italy and the western United States. Palaeogeogr., Palaeoclimatol., Palaeoecol. 93, 21-46.

Xie S., Pancost R., Huang J., Wignall P., Yu J., Tang X., Chen L., Huang X., Lai X., 2007. Changes in the global carbon cycle occurred as two episodes during the Permian-Triassic crisis. Geology 35, 1083-1086.

Xie S., Pancost R., Yin H., Wang H., Evershed R., 2005. Two episodes of microbial change coupled with Permo/Triassic faunal mass extinction. Nature 434, 494-497. 
1.

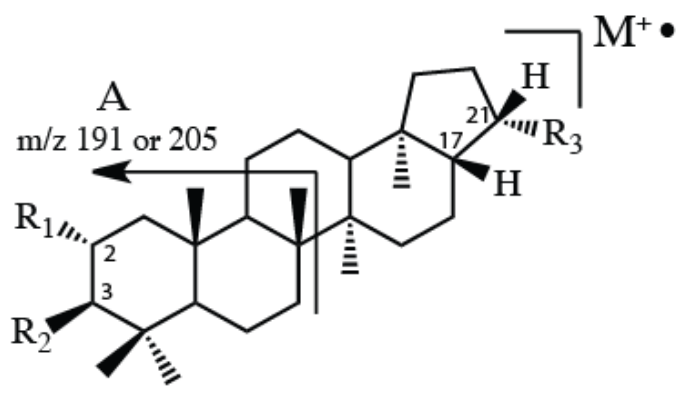

$\mathrm{R}_{1}=\mathrm{CH}_{3}, 2 \alpha(\mathrm{Me}), 17 \beta(\mathrm{H}), 21 \beta(\mathrm{H})$-hopane; Fragment $\mathrm{A} \mathrm{m} / \mathrm{z}=205$

$\mathrm{R}_{2}=\mathrm{CH}_{3}, 3 \beta(\mathrm{Me}), 17 \beta(\mathrm{H}), 21 \beta(\mathrm{H})$-hopane; Fragment $\mathrm{A} \mathrm{m} / \mathrm{z}=205$

$\mathrm{R}_{1}=\mathrm{R}_{2}=\mathrm{H}, 17 \beta(\mathrm{H}), 21 \beta(\mathrm{H})$-hopane; Fragment $\mathrm{A} \mathrm{m} / \mathrm{z}=191$

$\mathrm{R}_{3}=Y^{\left(\mathrm{CH}_{2}\right)_{\mathrm{n}} \mathrm{CH}_{3}} \mathrm{n}=0-5$

3.

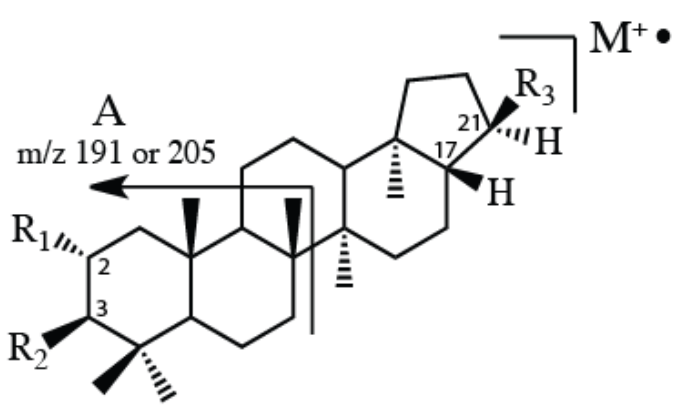

$\mathrm{R}_{1}=\mathrm{CH}_{3}, 2 \alpha(\mathrm{Me}), 17 \beta(\mathrm{H}), 21 \alpha(\mathrm{H})$-hopane; Fragment $\mathrm{A} \mathrm{m} / \mathrm{z}=205$

$\mathrm{R}_{2}=\mathrm{CH}_{3}, 3 \beta(\mathrm{Me}), 17 \beta(\mathrm{H}), 21 \alpha(\mathrm{H})$-hopane; Fragment $\mathrm{A} \mathrm{m} / \mathrm{z}=205$

$R_{1}=R_{2}=H, 17 \beta(H), 21 \alpha(H)$-hopane; Fragment A m/z=191

$\mathrm{R}_{3}=Y_{\mathrm{n}=0-5}^{\left(\mathrm{CH}_{2}\right)_{\mathrm{n}} \mathrm{CH}_{3}}$
2.

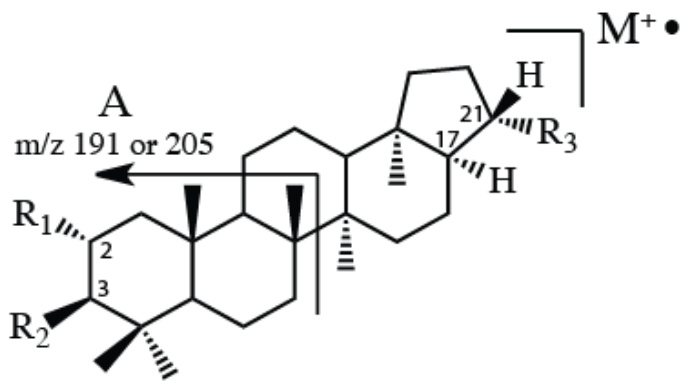

$\mathrm{R}_{1}=\mathrm{CH}_{3}, 2 \alpha(\mathrm{Me}), 17 \alpha(\mathrm{H}), 21 \beta(\mathrm{H})$-hopane; Fragment $\mathrm{A} \mathrm{m} / \mathrm{z}=205$

$\mathrm{R}_{2}=\mathrm{CH}_{3}, 3 \beta(\mathrm{Me}), 17 \alpha(\mathrm{H}), 21 \beta(\mathrm{H})$-hopane; Fragment $\mathrm{A} \mathrm{m} / \mathrm{z}=205$

$\mathrm{R}_{1}=\mathrm{R}_{2}=\mathrm{H}, 17 \alpha(\mathrm{H}), 21 \beta(\mathrm{H})$-hopane; Fragment A m/z=191

$\mathrm{R}_{3}=Y_{\mathrm{n}=0-5}^{\left(\mathrm{CH}_{2}\right)_{\mathrm{n}} \mathrm{CH}_{3}}$

4.

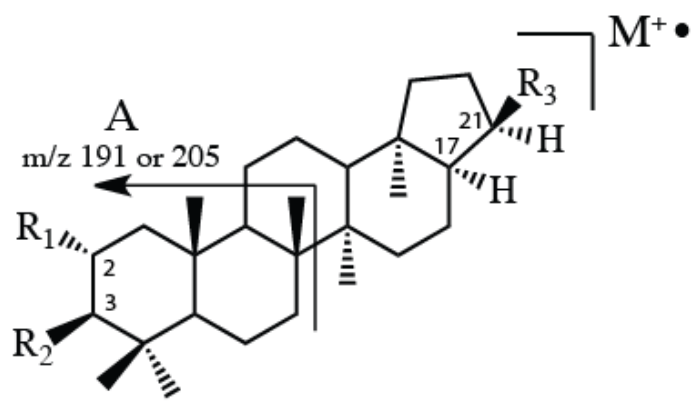

$\mathrm{R}_{1}=\mathrm{CH}_{3}, 2 \alpha(\mathrm{Me}), 17 \alpha(\mathrm{H}), 21 \alpha(\mathrm{H})$-hopane; Fragment $\mathrm{A} \mathrm{m} / \mathrm{z}=205$

$\mathrm{R}_{2}=\mathrm{CH}_{3}, 3 \beta(\mathrm{Me}), 17 \alpha(\mathrm{H}), 21 \alpha(\mathrm{H})$-hopane; Fragment $\mathrm{A} \mathrm{m} / \mathrm{z}=205$

$\mathrm{R}_{1}=\mathrm{R}_{2}=\mathrm{H}, 17 \alpha(\mathrm{H}), 21 \alpha(\mathrm{H})$-hopane; Fragment A m/z=191

$\mathrm{R}_{3}=Y_{\mathrm{n}=0-5}^{\left(\mathrm{CH}_{2}\right)_{\mathrm{n}} \mathrm{CH}_{3}}$

Figure. 1. Structures of the $\beta \beta$-hopanoid (1), $\alpha \beta$-hopanoid (2), $\beta \alpha$-hopanoid (3), and the $\alpha \alpha$-hopanoid (4) series are illustrated. Key asymmetric carbon atoms of interest are labeled. The mass spectrometry fragment A is marked with the expected mass units depending on methylation. The molecular ion is indicated and has an expected mass of 412 for $\mathrm{C}_{30}$ hopane up to 482 for $\mathrm{C}_{35}$ hopanes. 

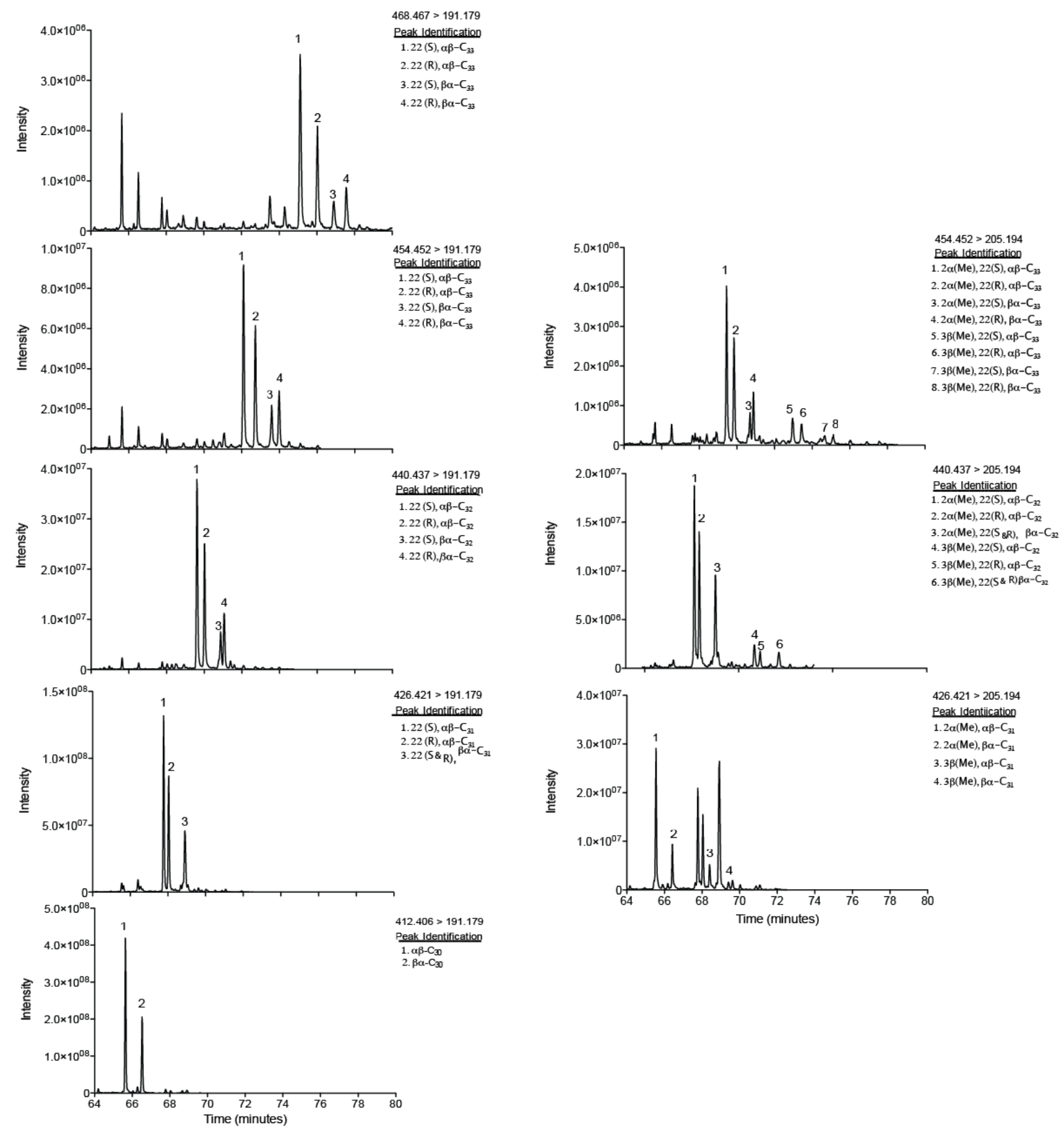

Figure 2. Multiple reaction monitoring (MRM) chromatograms from sample MScore1- 35-1 at $94.53 \mathrm{~m}$ depth are shown. The MRM transitions are written to the right of the chromatogram. In the left column, the 191.179 fragment corresponds to the A/B rings for homohopanes (see Fig. 1). In the right column, the 205 mass fragment corresponds to the $\mathrm{A} / \mathrm{B}$ ring fragment for $2 \alpha$ - and $3 \beta$-methylhomohopanes. All peaks are marked numerically with the corresponding identification to the right. 


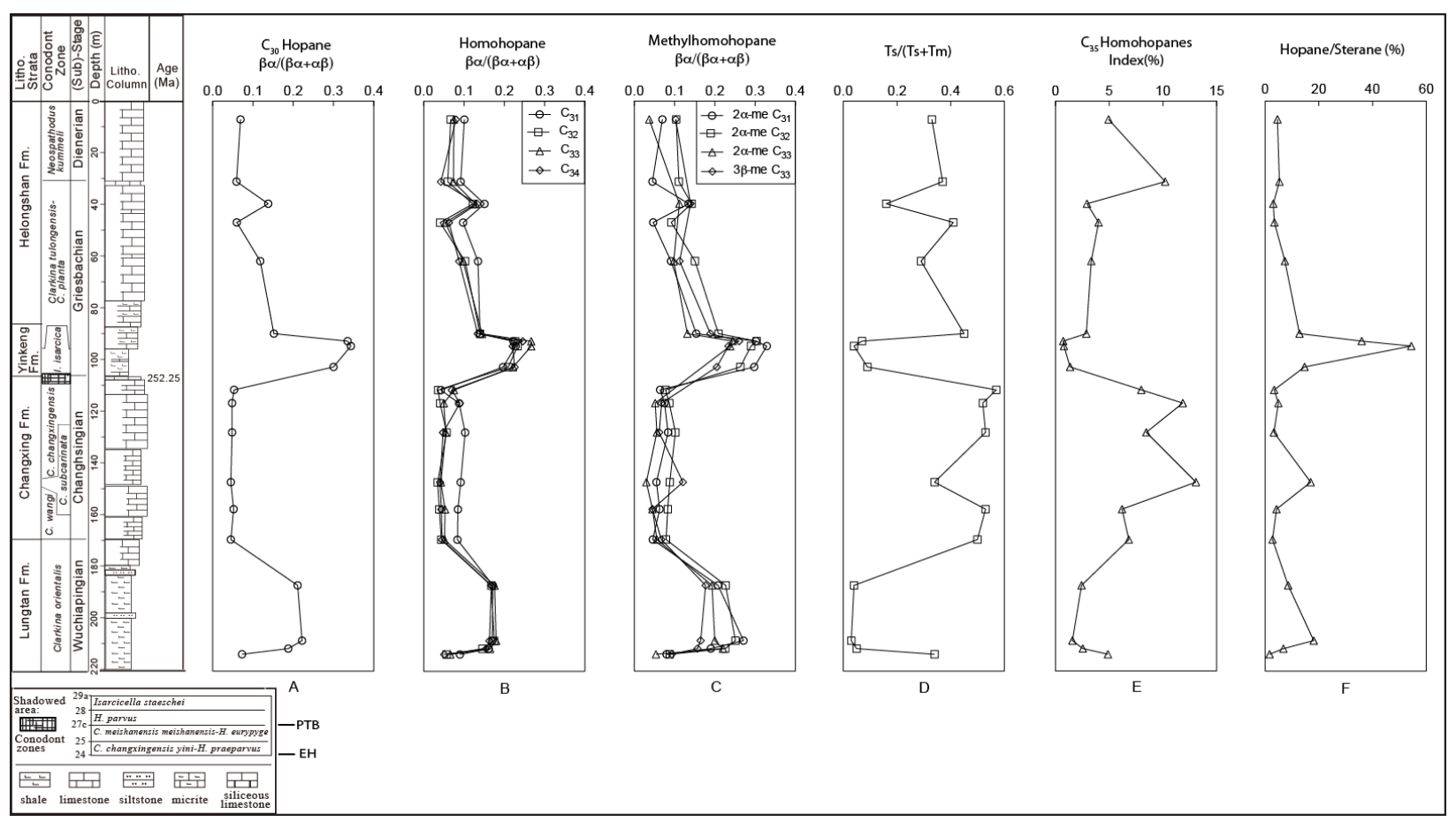

Figure 3. Vertical profiles of molecular parameters, including the moretane/hopane ratio $(\beta \alpha /(\beta \alpha+\alpha \beta))$ of $\mathrm{C}_{30}(\mathrm{~A})$, the homohopane series (B), the $2 \alpha$ - and $3 \beta$ methylhomohopanes (C), Ts/(Ts+Tm) (D), the $\mathrm{C}_{35}$ homohopane index (\%) (E), and hopane/sterane $(\%)(\mathrm{F})$, are plotted versus the stratigraphic column (modified from Cao et al., 2009). The Ts/Ts+Tm, $\mathrm{C}_{35} \mathrm{HHI}$, and hopane/sterane data are from Cao et al., 2009. The three moretane/hopane excursions occur at $39.56 \mathrm{~m}, 92.61-102.59 \mathrm{~m}$, and 186.91$213.52 \mathrm{~m}$. The legend for the stratigraphic column is at bottom right. In the expanded view of the conodont zones in the legend, the PTB is marked according to the first appearance of the conodont Hindeodus parvus, and the extinction horizon (EH) is marked at the top of bed 24 . 


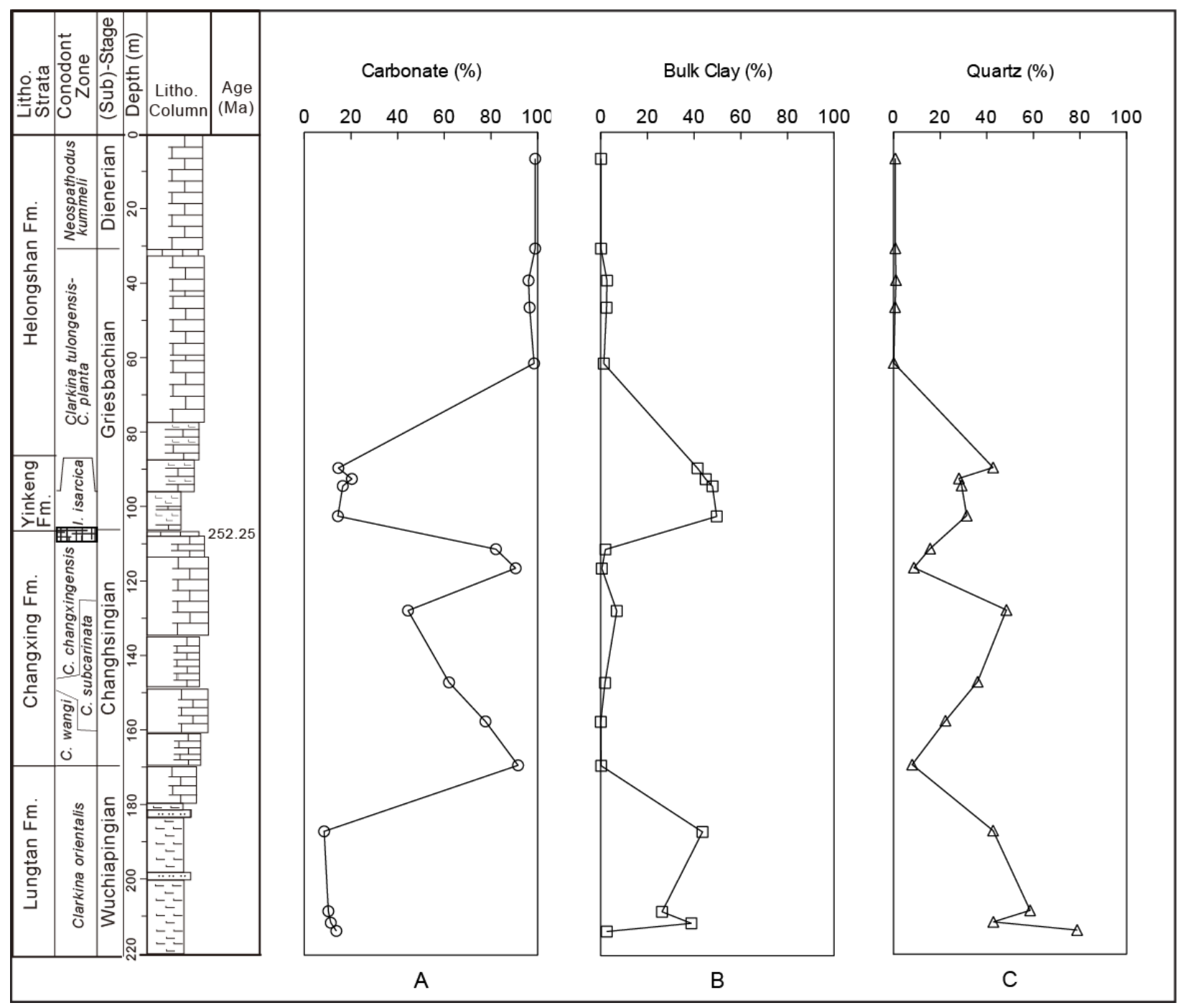

Figure 4. Vertical profiles of the bulk lithological components by percentage versus the stratigraphic column (modified from Cao et al., 2009). A) percentage carbonate; B) percentage total clay; C) percentage quartz. See figure 3 for the stratigraphic column legend. 

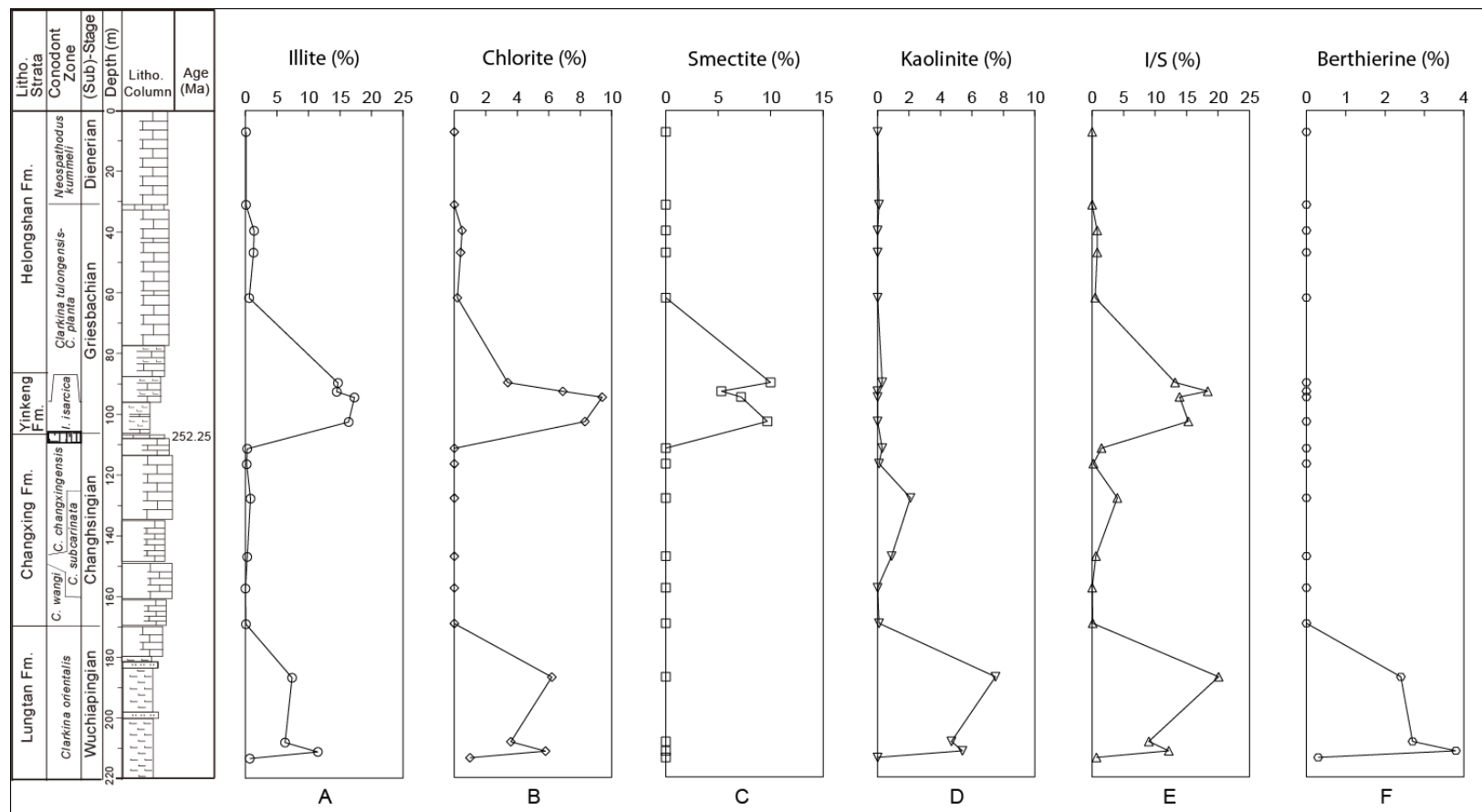

Figure 5. Vertical profiles of clay types plotted against the stratigraphic column (modified from Cao et al. 2009). A) Absolute percent illite; B) absolute percent chlorite; C) absolute percent smectite; D) absolute percent kaolinite; E) absolute percent mixed layer illite/smectite (I/S); F) absolute percent berthierine. Note the difference in the $\mathrm{x}$ axis scale for clay types. See figure 3 for the stratigraphic column legend. 

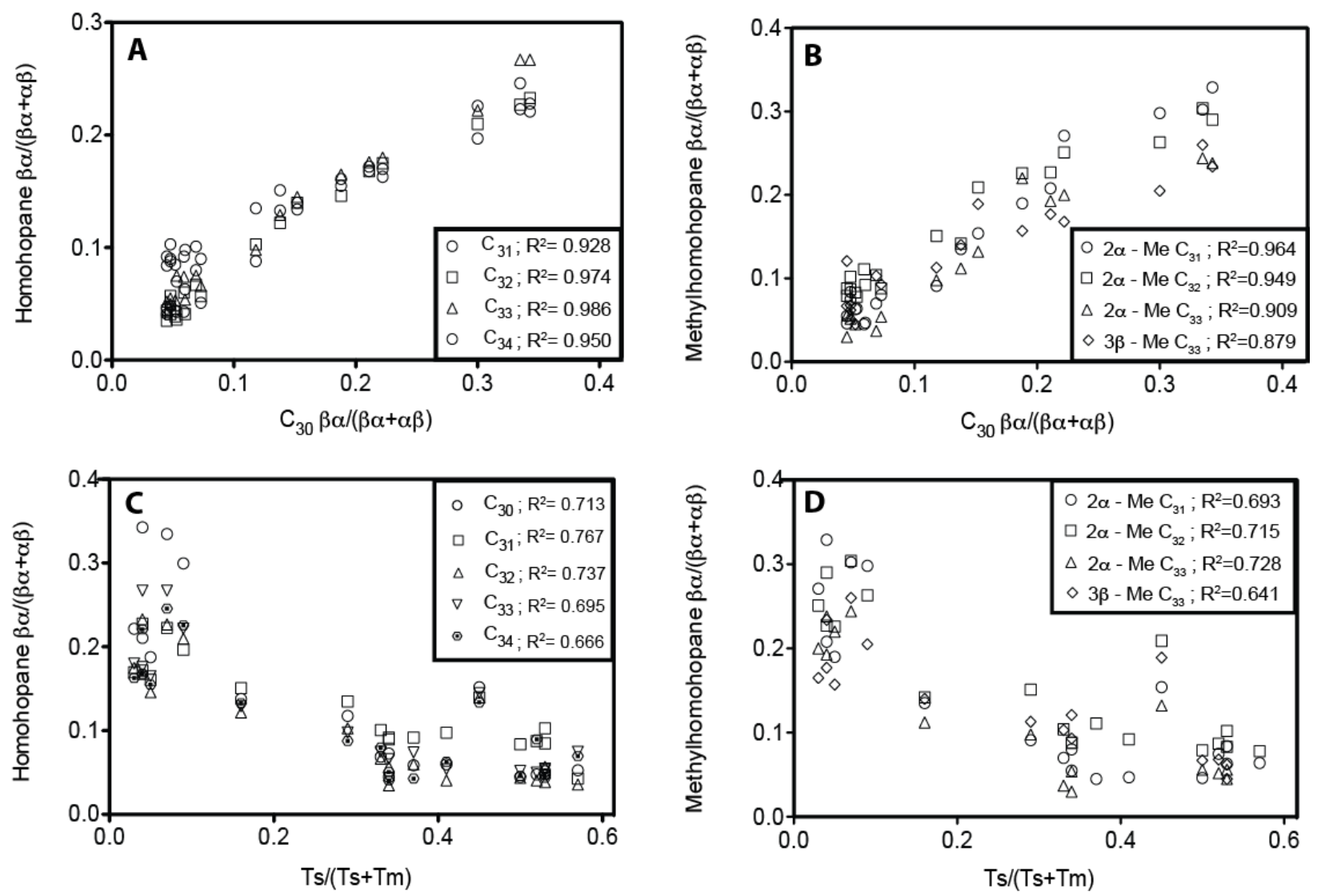

Figure 6. Cross-correlations of molecular indicies. Plots A and B relate moretane/hopane of the $\mathrm{C}_{30}$ hopane to the higher homologues of the homohopane series and $2 \alpha-\& 3 \beta$ - methylhomohopane series, respectively. Plots $\mathrm{C}$ and D relate Ts /(Ts+Tm) to the higher homologues of the homohopane series and $2 \alpha-$ and $3 \beta-$ methylhomohopane series, respectively. The $\mathrm{R}^{2}$ value associated with the linear regression for each series relationship is given in the legend. 

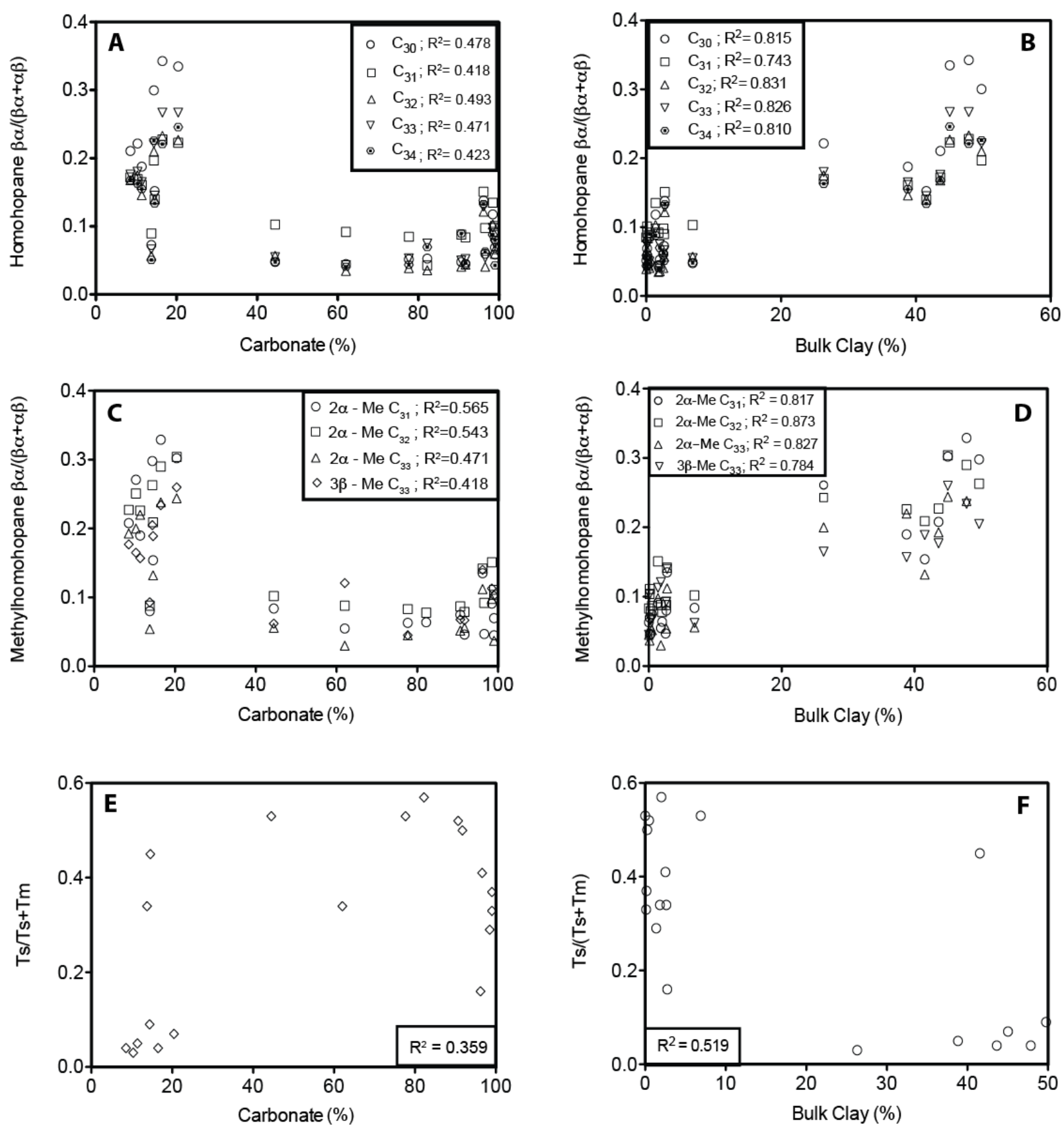

Figure 7. Cross-correlations of molecular and bulk lithological data. Plots A and B relate carbonate weight percentage and total bulk clay to the homohopane series, respectively. Plots $\mathrm{C}$ and $\mathrm{D}$ relate carbonate weight percentage and total bulk clay to the $2 \alpha$ - and $3 \beta-$ methylhomohopane series. Plots $\mathrm{E}$ and F relate carbonate weight percentage and total bulk clay to $\mathrm{Ts} /(\mathrm{Ts}+\mathrm{Tm})$. 


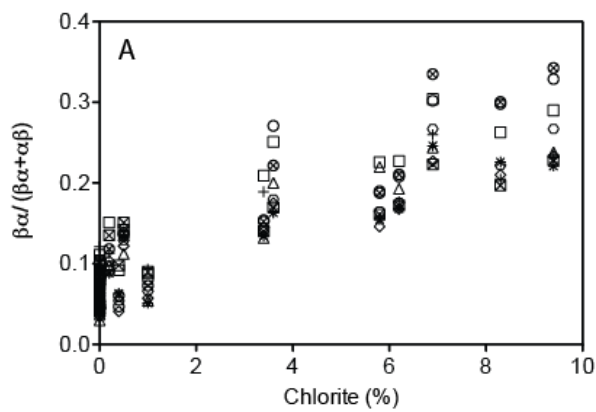

(2 $\mathrm{C}_{30} ; \mathrm{R}^{2}=0.901$

$\otimes \mathrm{C}_{31} ; \mathrm{R}^{2}=0.806$

$\mathrm{C}_{32} ; \mathrm{R}^{2}=0.863$

- $\mathrm{C}_{33} ; \mathrm{R}^{2}=0.884$

* $\mathrm{C}_{34} ; \mathrm{R}^{2}=0.851$

- $2 \alpha-\mathrm{me} \mathrm{C}_{31} ; \mathrm{R}^{2}=0.881$

- $2 \alpha-$ me $_{32} ; R^{2}=0.866$

$\Delta 2 \alpha$-me $C_{33} ; R^{2}=0.871$

$+\quad 3 \beta$-me $C_{33} ; R^{2}=0.773$

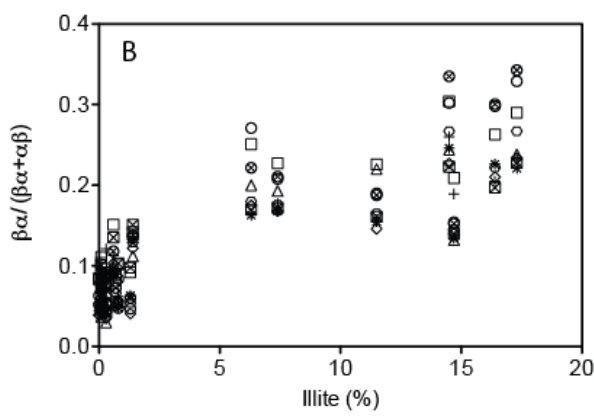

(2) $\mathrm{C}_{30} ; \mathrm{R}^{2}=0.814$

$\otimes C_{31} ; R^{2}=0.731$

- $C_{32} ; R^{2}=0.807$

- $\mathrm{C}_{33} ; \mathrm{R}^{2}=0.821$

* $\mathrm{C}_{34} ; \mathrm{R}^{2}=0.796$

- $2 \alpha$-me $C_{31} ; R^{2}=0.788$

ㅁ $2 \alpha-\mathrm{me} \mathrm{C}_{32} ; \mathrm{R}^{2}=0.845$

$\Delta \quad 2 \alpha-$ me C $_{33} ; R^{2}=0.752$

$+\quad 3 \beta-\mathrm{me} \mathrm{C}_{33} ; \mathrm{R}^{2}=0.799$

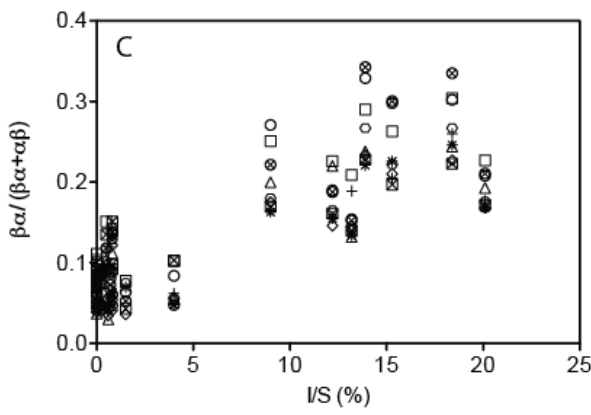

(2) $\mathrm{C}_{30} ; \mathrm{R}^{2}=0.752$

$\otimes \quad C_{31} ; R^{2}=0.692$

- $C_{32} ; R^{2}=0.773$

- $C_{33} ; R^{2}=0.769$

* $\mathrm{C}_{34} ; \mathrm{R}^{2}=0.763$

- $2 \alpha$-me $C_{31} ; R^{2}=0.750$

$\quad 2 \alpha$-me $C_{32} ; R^{2}=0.820$

$\Delta \quad 2 \alpha$-me $C_{33} ; R^{2}=0.772$

$+\quad 3 \beta-\mathrm{me} \mathrm{C}_{33} ; \mathrm{R}^{2}=0.731$
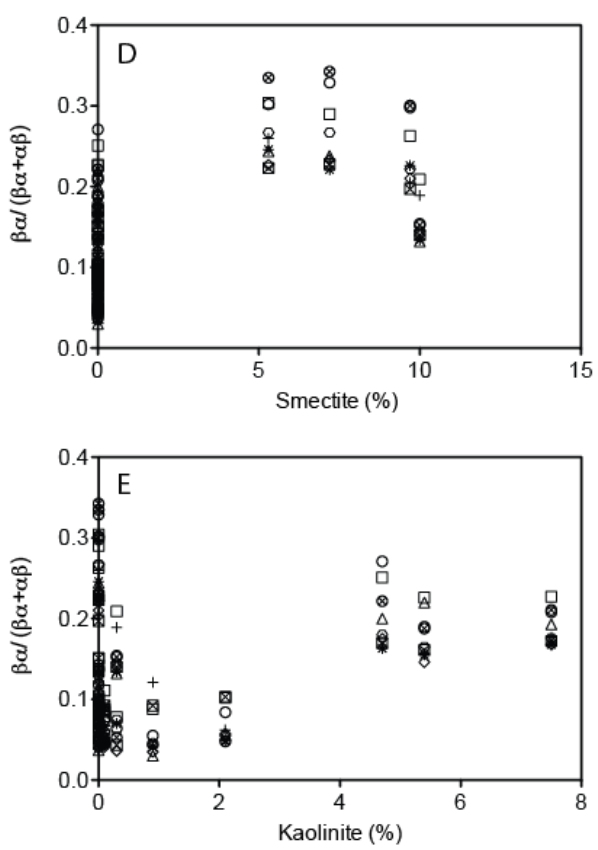

$C_{30} ; R^{2}=0.054$

$\otimes \quad C_{31} ; R^{2}=0.074$

$\mathrm{C}_{32} ; \mathrm{R}^{2}=0.087$

- $\mathrm{C}_{33} ; \mathrm{R}^{2}=0.063$

* $\mathrm{C}_{34} ; \mathrm{R}^{2}=0.064$

- $2 \alpha$-me $C_{31} ; R^{2}=0.096$

- $2 \alpha$-me $C_{32} ; R^{2}=0.127$

$\Delta 2 \alpha-\mathrm{me} \mathrm{C}_{33} ; \mathrm{R}^{2}=0.222$

$+\quad 3 \beta-\mathrm{me} \mathrm{C}_{33} ; \mathrm{R}^{2}=0.024$
Figure 8. Correlations between moretane/hopane ratios and the specific clay types are shown for the homohopane and $2 \alpha-$ and $3 \beta-$ methylhomohopane series. The $R^{2}$ values are listed in the legend next to the corresponding series. A) Absolute percent chlorite; B) absolute percent illite; C) absolute percent mixed layer illite/smectite (I/S); D) absolute percent smectite; E) absolute percent kaolinite. 

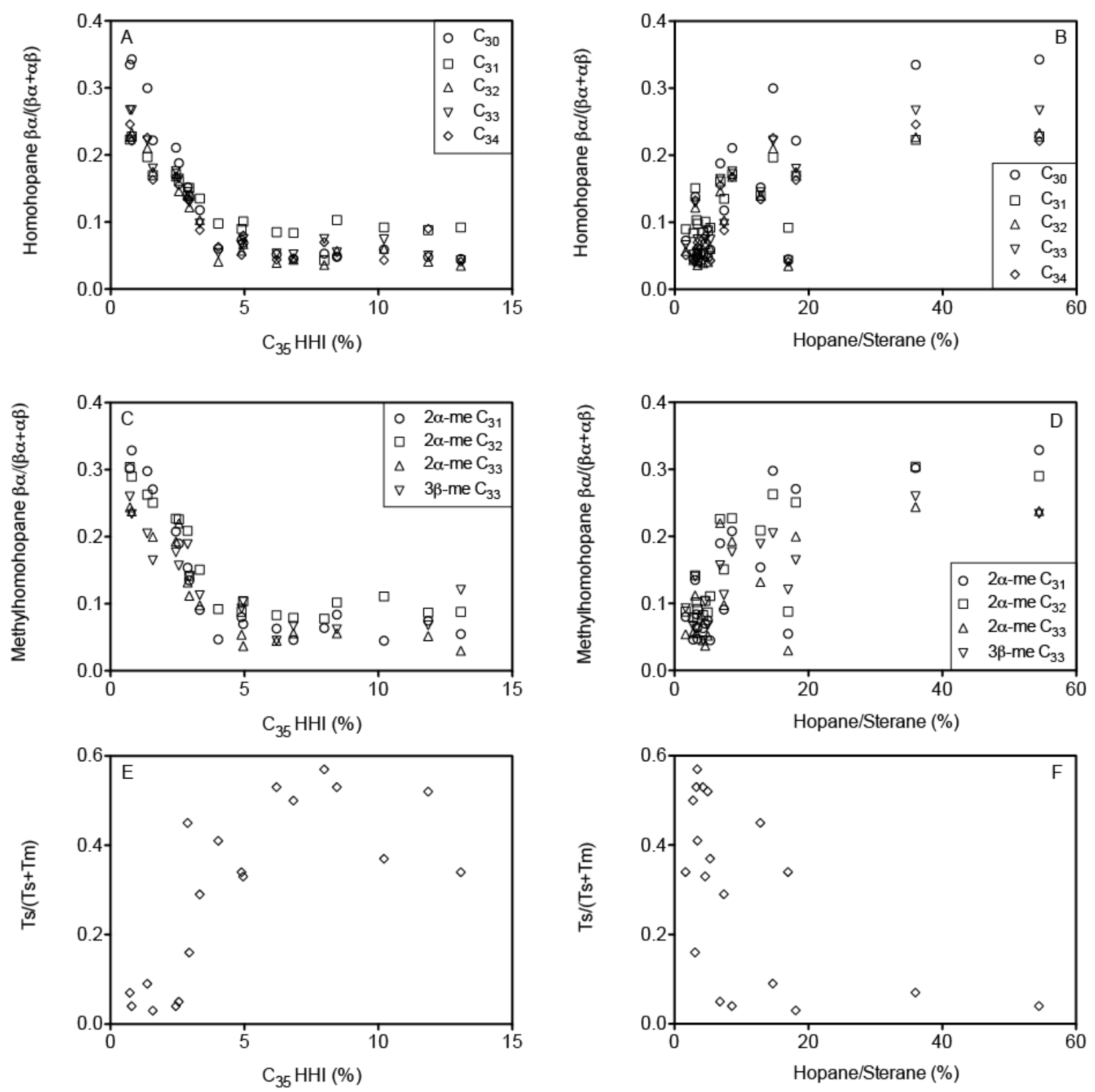

Figure 9. Correlations of geochemical source and redox indicators against moretane/hopane ratios and Ts/(Ts+Tm). C 35 HHI (\%) vs. homohopanes $\beta \alpha /(\beta \alpha+\alpha \beta)$ (A), methylhomohopanes $\beta \alpha /(\beta \alpha+\alpha \beta)(\mathrm{C})$, and Ts/(Ts+Tm) (E). Hopane/Sterane (\%) versus homohopanes $\beta \alpha /(\beta \alpha+\alpha \beta)$ (B), methylhomohopanes $\beta \alpha /(\beta \alpha+\alpha \beta)$ (D), and $\mathrm{Ts} /(\mathrm{Ts}+\mathrm{Tm})(\mathrm{F})$. 

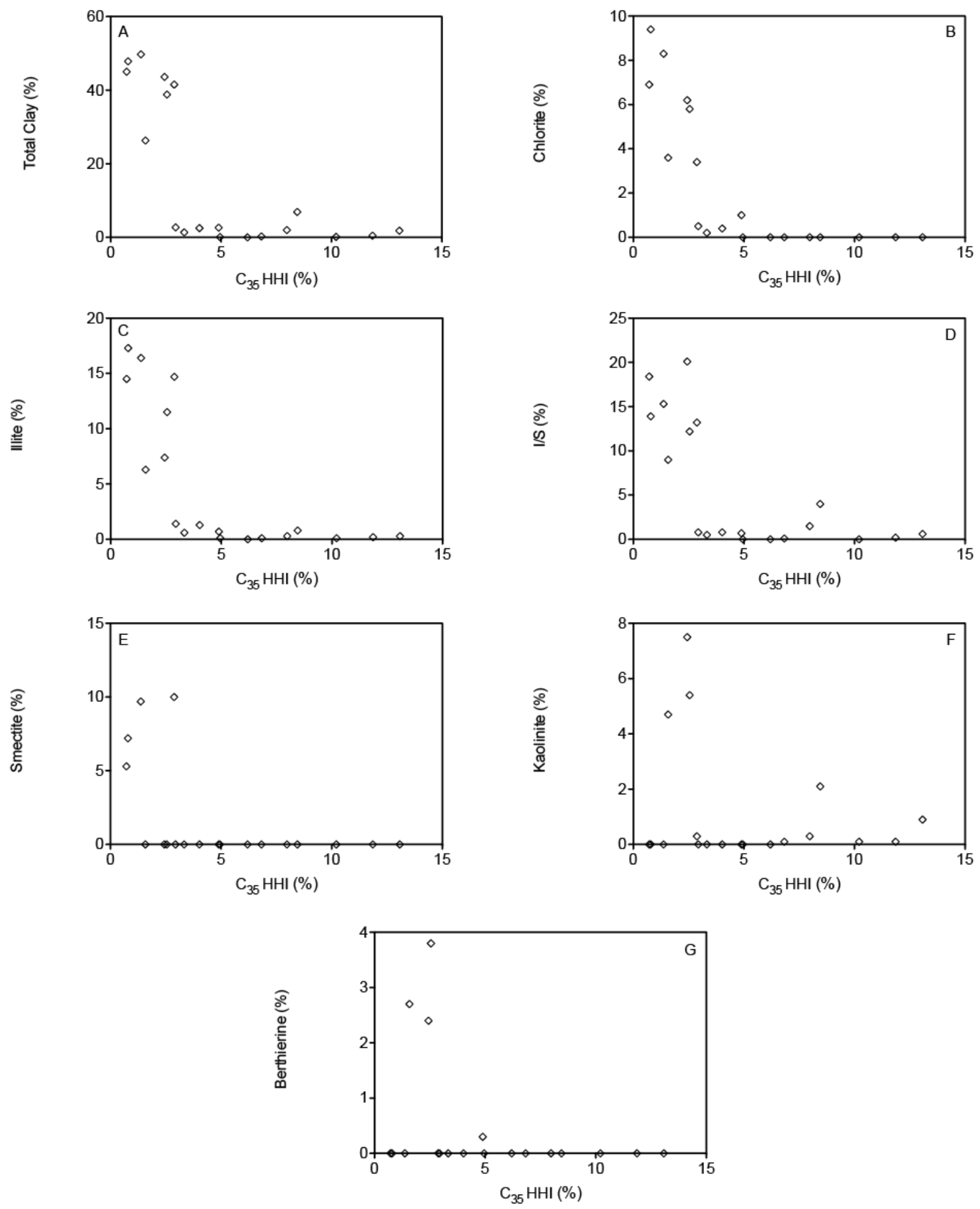

Figure 10. Correlations of $\mathrm{C}_{35} \mathrm{HHI}(\%)$, a redox indicator, against percent total clay and clay types. $\mathrm{C}_{35} \mathrm{HHI}(\%)$ vs. percent total clay (A), absolute percent chlorite (B), absolute percent illite $(\mathrm{C})$, absolute percent illite/smectite mixed layer clay (I/S) (D), absolute percent smectite (E), absolute percent kaolinite $(\mathrm{F})$, absolute percent berthierine (G). 

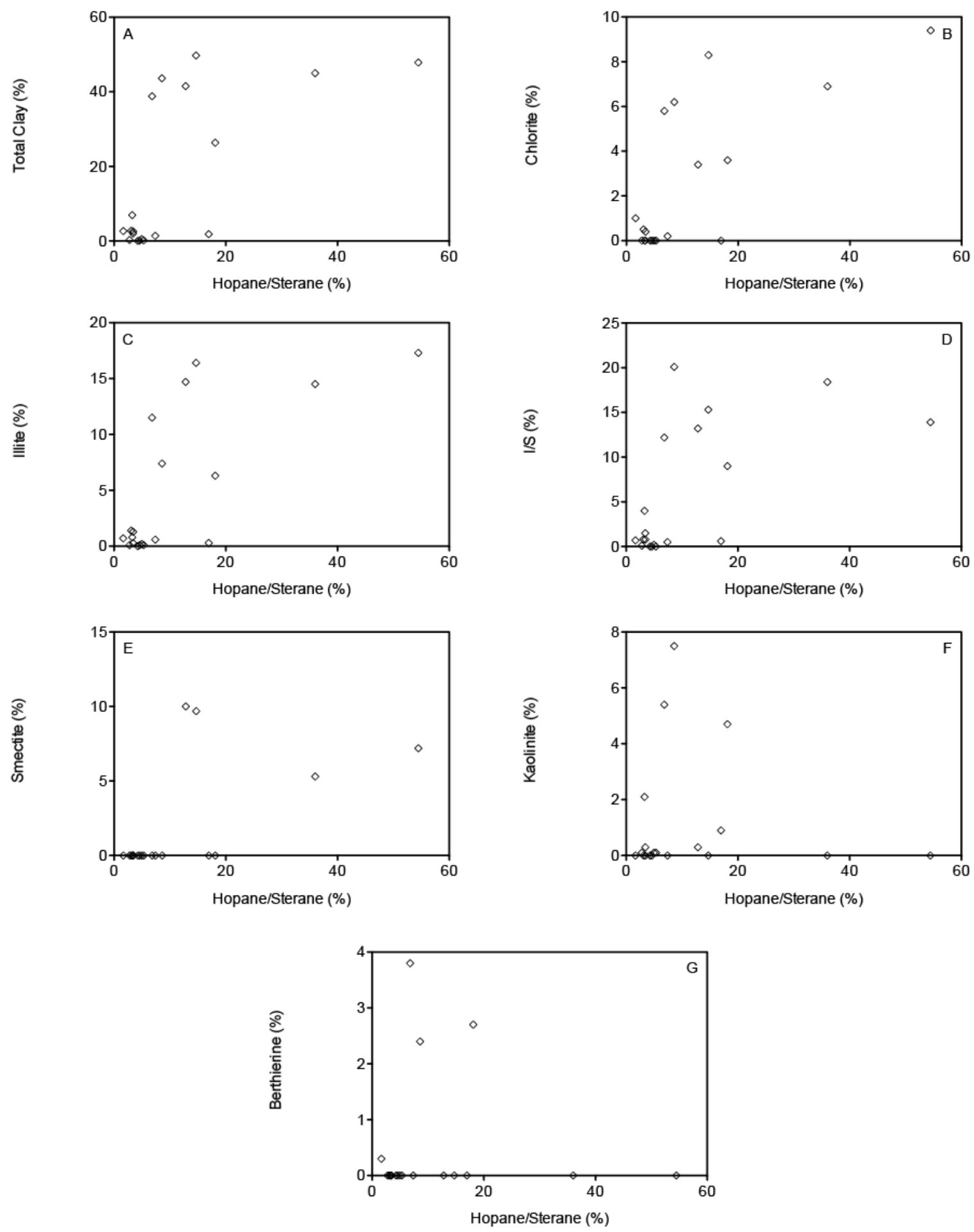

Figure 11. Correlations of Hopane/Sterane (\%), a geochemical source indicator, against percent total clay and clay types. Hopane/Sterane (\%) vs. percent total clay (A), absolute percent chlorite (B), absolute percent illite (C), absolute percent illite/smectite mixed layer clay $(\mathrm{I} / \mathrm{S})(\mathrm{D})$, absolute percent smectite $(\mathrm{E})$, absolute percent kaolinite $(\mathrm{F})$, absolute percent berthierine $(\mathrm{G})$. 
Table 1. Summary of geochemical data

\begin{tabular}{|c|c|c|c|c|c|c|c|c|c|c|}
\hline $\begin{array}{l}\text { Sample } \\
\text { ID }\end{array}$ & $\begin{array}{c}\text { Drilling } \\
\text { Depth } \\
(\mathrm{m})\end{array}$ & $\begin{array}{c}C_{30} \\
\beta \alpha /(\beta \alpha+ \\
\alpha \beta)\end{array}$ & $\begin{array}{c}\mathrm{C}_{31} \\
\beta \alpha /(\beta \alpha+ \\
\alpha \beta)\end{array}$ & $\begin{array}{c}\mathrm{C}_{32} \\
\beta \alpha /(\beta \alpha+ \\
\alpha \beta)\end{array}$ & $\begin{array}{c}\mathrm{C}_{33} \\
\beta \alpha /(\beta \alpha+ \\
\alpha \beta)\end{array}$ & $\begin{array}{c}\mathrm{C}_{34} \\
\beta \alpha /(\beta \alpha+ \\
\alpha \beta)\end{array}$ & $\begin{array}{c}2 \alpha \mathrm{Me} \mathrm{C}{ }_{31} \\
\beta \alpha /(\beta \alpha+\alpha \beta)\end{array}$ & $\begin{array}{c}2 \alpha \mathrm{Me} \mathrm{C} \mathrm{C}_{32} \\
\beta \alpha /(\beta \alpha+\alpha \beta)\end{array}$ & $\begin{array}{c}2 \alpha \mathrm{Me} \mathrm{C} \mathrm{C}_{33} \\
\beta \alpha /(\beta \alpha+\alpha \beta)\end{array}$ & $\begin{array}{c}3 \beta \mathrm{Me} \mathrm{C}{ }_{33} \\
\beta \alpha /(\beta \alpha+\alpha \beta)\end{array}$ \\
\hline MS05-140 & 7.05 & 0.069 & 0.101 & 0.067 & 0.075 & 0.080 & 0.070 & 0.104 & 0.037 & 0.103 \\
\hline MS05-165 & 31.05 & 0.059 & 0.092 & 0.060 & 0.074 & 0.043 & 0.045 & 0.111 & - & - \\
\hline MS05-170 & 39.56 & 0.138 & 0.151 & 0.122 & 0.129 & 0.133 & 0.135 & 0.142 & 0.112 & 0.140 \\
\hline MS05-175 & 46.79 & 0.060 & 0.098 & 0.041 & 0.054 & 0.063 & 0.047 & 0.092 & - & - \\
\hline MS05-180 & 61.77 & 0.118 & 0.135 & 0.103 & 0.098 & 0.088 & 0.091 & 0.151 & 0.098 & 0.113 \\
\hline MS05-200 & 89.71 & 0.152 & 0.140 & 0.140 & 0.145 & 0.134 & 0.154 & 0.209 & 0.132 & 0.189 \\
\hline $\begin{array}{c}\text { MS-1- } \\
\text { core 36-3 }\end{array}$ & 92.61 & 0.335 & 0.223 & 0.227 & 0.267 & 0.246 & 0.302 & 0.304 & 0.244 & 0.260 \\
\hline $\begin{array}{l}\text { MS-1- } \\
\text { core 35-1 }\end{array}$ & 94.53 & 0.343 & 0.228 & 0.233 & 0.267 & 0.221 & 0.329 & 0.290 & 0.238 & 0.234 \\
\hline $\begin{array}{c}\text { MS-1- } \\
\text { core 34-3 }\end{array}$ & 102.59 & 0.300 & 0.197 & 0.210 & 0.222 & 0.226 & 0.298 & 0.263 & - & 0.205 \\
\hline MS05-2b & 111.44 & 0.053 & 0.043 & 0.036 & 0.075 & 0.070 & 0.064 & 0.078 & - & - \\
\hline MS05-10 & 116.51 & 0.048 & 0.088 & 0.041 & 0.050 & 0.090 & 0.075 & 0.087 & 0.052 & 0.068 \\
\hline MS05-30 & 127.82 & 0.048 & 0.103 & 0.057 & 0.055 & 0.048 & 0.084 & 0.102 & 0.056 & 0.062 \\
\hline MS05-60 & 147.08 & 0.045 & 0.092 & 0.035 & 0.043 & 0.041 & 0.055 & 0.088 & 0.030 & 0.121 \\
\hline MS05-76 & 157.48 & 0.052 & 0.085 & 0.039 & 0.053 & 0.044 & 0.063 & 0.083 & 0.045 & 0.045 \\
\hline MS05-92b & 169.24 & 0.045 & 0.084 & 0.044 & 0.052 & 0.046 & 0.046 & 0.079 & 0.056 & 0.067 \\
\hline MS05-117 & 186.91 & 0.211 & 0.172 & 0.168 & 0.176 & 0.168 & 0.208 & 0.227 & 0.193 & 0.177 \\
\hline MS05-134 & 208.22 & 0.222 & 0.170 & 0.175 & 0.180 & 0.163 & 0.271 & 0.251 & 0.200 & 0.165 \\
\hline $\begin{array}{c}\text { MS05- } \\
136 c\end{array}$ & 211.3 & 0.188 & 0.161 & 0.146 & 0.165 & 0.155 & 0.190 & 0.226 & 0.220 & 0.157 \\
\hline MS05-138 & 213.52 & 0.073 & 0.090 & 0.057 & 0.066 & 0.051 & 0.080 & 0.088 & 0.054 & 0.093 \\
\hline
\end{tabular}


Table 2. Bulk lithology

\begin{tabular}{lcccc}
\hline \multicolumn{1}{c}{ Sample ID } & $\begin{array}{c}\text { Percent } \\
\text { Clay }\end{array}$ & $\begin{array}{c}\text { Percent } \\
\text { Carbonate }\end{array}$ & $\begin{array}{c}\text { Percent } \\
\text { Quartz }\end{array}$ & $\begin{array}{c}\text { Percent } \\
\text { Other }\end{array}$ \\
\hline MS05-140 & & & & \\
MS05-165 & 0.12 & 99.0 & 0.85 & 0.00 \\
MS05-170 & 2.16 & 99.0 & 0.82 & 0.00 \\
MS05-175 & 2.52 & 96.2 & 1.07 & 0.00 \\
MS05-180 & 1.37 & 96.6 & 0.74 & 0.13 \\
MS05-200 & 41.6 & 14.6 & 0.11 & 0.04 \\
MS-1-core 36-3 & 45.0 & 20.4 & 28.1 & 1.11 \\
MS-1-core 35-1 & 47.9 & 16.5 & 29.3 & 6.41 \\
MS-1-core 34-3 & 49.8 & 14.4 & 31.4 & 4.38 \\
MS05-2b & 2.01 & 82.2 & 15.8 & 0.00 \\
MS05-10 & 0.49 & 90.7 & 8.84 & 0.00 \\
MS05-30 & 6.90 & 44.5 & 48.5 & 0.12 \\
MS05-60 & 1.83 & 62.0 & 36.1 & 0.05 \\
MS05-76 & 0.00 & 77.7 & 22.4 & 0.00 \\
MS05-92b & 0.27 & 91.7 & 8.03 & 0.00 \\
MS05-117 & 43.6 & 8.54 & 42.8 & 5.00 \\
MS05-134 & 26.3 & 10.3 & 58.6 & 4.71 \\
MS05-136c & 38.8 & 11.4 & 42.8 & 6.93 \\
MS05-138 & 2.64 & 13.8 & 78.9 & 4.74 \\
& & & & \\
\hline
\end{tabular}


Table 3. Absolute and relative clay type percentages

\begin{tabular}{lcccccc}
\hline \multicolumn{1}{c}{ Sample ID } & $\begin{array}{c}\text { Absolute } \\
\text { Percent } \\
\text { Illite }\end{array}$ & $\begin{array}{c}\text { Absolute } \\
\text { Percent } \\
\text { Berthierine }\end{array}$ & $\begin{array}{c}\text { Absolute } \\
\text { Percent } \\
\text { Kaolinite }\end{array}$ & $\begin{array}{c}\text { Absolute } \\
\text { Percent } \\
\text { Chlorite }\end{array}$ & $\begin{array}{c}\text { Absolute } \\
\text { Percent } \\
\text { Illite/Smectite } \\
\text { Mixed Layer }\end{array}$ & $\begin{array}{c}\text { Absolute } \\
\text { Percent } \\
\text { Smectite }\end{array}$ \\
\hline MS05-140 & 0.07 & 0.00 & 0.01 & 0.01 & 0.04 & 0.00 \\
MS05-165 & 0.06 & 0.00 & 0.06 & 0.00 & 0.04 & 0.00 \\
MS05-170 & 1.45 & 0.00 & 0.00 & 0.48 & 0.81 & $<0.01$ \\
MS05-175 & 1.30 & 0.00 & 0.01 & 0.42 & 0.79 & $<0.01$ \\
MS05-180 & 0.63 & 0.00 & 0.00 & 0.20 & 0.53 & $<0.01$ \\
ms05-200 & 14.7 & 0.00 & 0.27 & 3.38 & 13.2 & 9.96 \\
ms-1-core 36-3 & 14.5 & 0.00 & 0.00 & 6.87 & 18.4 & 5.27 \\
ms-1-core 35-1 & 17.3 & 0.00 & 0.00 & 9.38 & 13.9 & 7.21 \\
ms-1-core 34-3 & 16.4 & 0.00 & 0.01 & 8.31 & 15.3 & 9.73 \\
ms05-2b & 0.26 & 0.00 & 0.25 & 0.00 & 1.49 & 0.00 \\
MS05-10 & 0.25 & 0.00 & 0.07 & 0.00 & 0.17 & 0.00 \\
MS05-30 & 0.76 & 0.00 & 2.14 & 0.00 & 4.00 & 0.00 \\
MS05-60 & 0.28 & 0.00 & 0.91 & 0.00 & 0.65 & 0.00 \\
MS05-76 & 0.00 & 0.00 & 0.00 & 0.00 & 0.00 & 0.00 \\
MS05-92b & 0.11 & 0.00 & 0.06 & 0.00 & 0.11 & 0.00 \\
MS05-117 & 7.40 & 2.43 & 7.53 & 6.21 & 20.1 & 0.02 \\
MS05-134 & 6.34 & 2.67 & 4.75 & 3.56 & 9.00 & 0.01 \\
MS05-136c & 11.5 & 3.83 & 5.41 & 5.84 & 12.2 & 0.02 \\
MS05-138 & 0.68 & 0.30 & 0.01 & 0.96 & 0.69 & 0.00 \\
\hline
\end{tabular}

\begin{tabular}{lcccccc}
\hline \multicolumn{1}{c}{ Sample ID } & $\begin{array}{c}\text { Relative } \\
\text { Percent } \\
\text { Illite }\end{array}$ & $\begin{array}{c}\text { Relative } \\
\text { Percent } \\
\text { Berthierine }\end{array}$ & $\begin{array}{c}\text { Relative } \\
\text { Percent } \\
\text { Kaolinite }\end{array}$ & $\begin{array}{c}\text { Relative } \\
\text { Percent } \\
\text { Chlorite }\end{array}$ & $\begin{array}{c}\text { Relative } \\
\text { Percent } \\
\text { Illite/Smectite } \\
\text { Mixed Layer }\end{array}$ & $\begin{array}{c}\text { Relative } \\
\text { Percent } \\
\text { Smectite }\end{array}$ \\
\hline MS05-140 & 54.4 & 0.00 & 10.7 & 4.43 & 30.5 & 0.03 \\
MS05-165 & 35.8 & 0.00 & 37.2 & 0.00 & 27.0 & 0.00 \\
MS05-170 & 53.0 & 0.00 & 0.00 & 17.4 & 29.5 & 0.14 \\
MS05-175 & 51.7 & 0.00 & 0.32 & 16.8 & 31.2 & 0.00 \\
MS05-180 & 46.3 & 0.00 & 0.00 & 14.9 & 38.7 & 0.08 \\
ms05-200 & 35.4 & 0.00 & 0.65 & 8.14 & 31.9 & 24.0 \\
ms-1-core 36-3 & 32.3 & 0.00 & 0.00 & 15.3 & 40.8 & 11.7 \\
ms-1-core 35-1 & 36.2 & 0.00 & 0.00 & 19.6 & 29.1 & 15.1 \\
ms-1-core 34-3 & 33.0 & 0.00 & 0.01 & 16.7 & 30.7 & 19.6 \\
ms05-2b & 13.0 & 0.00 & 12.5 & 0.00 & 74.4 & 0.00 \\
MS05-10 & 51.0 & 0.00 & 13.3 & 0.00 & 35.7 & 0.00 \\
MS05-30 & 11.0 & 0.00 & 31.0 & 0.00 & 58.0 & 0.00 \\
MS05-60 & 15.3 & 0.00 & 49.4 & 0.00 & 35.3 & 0.00 \\
MS05-76 & 48.3 & 0.00 & 17.6 & 0.00 & 34.1 & 0.00 \\
MS05-92b & 38.6 & 0.00 & 20.1 & 0.00 & 41.2 & 0.15 \\
MS05-117 & 16.9 & 5.56 & 17.3 & 14.2 & 46.0 & 0.05 \\
MS05-134 & 24.1 & 10.1 & 18.0 & 13.5 & 34.2 & 0.05 \\
MS05-136c & 29.7 & 9.87 & 13.9 & 15.0 & 31.4 & 0.05 \\
MS05-138 & 25.8 & 11.4 & 0.36 & 36.4 & 26.1 & 0.00 \\
\hline
\end{tabular}




\section{Chapter 7:}

\section{Concluding Remarks}

The previous chapters have discussed the fidelity and application of multiple geochemical tools that have provided the basis for illuminating aspects of Earth's redox evolution, particularly the origin of oxygenic photosynthesis and the occurrence of photic zone euxinia. The finding that currently existing hydrocarbon biomarkers in Archean rocks were introduced via contamination affects the late Archean sequence of events preceding the GOE. At present, we cannot know whether hopanes or steranes were deposited in Archean rocks because the thermal maturity of the currently characterized rocks is too high, thereby concealing the organic precursor compounds. While inorganic evidence has been used to suggest whiffs of oxygen and oxygen oases as indirect evidence of oxygenic photosynthesis hundreds of millions of years prior to the GOE (e.g. 1-3), some of this inorganic evidence for pre-GOE oxygen production has also recently come under question (4). A different line of evidence based on fossilized bubbles trapped in conical stromatolites could record photosynthetic production of oxygen as early as $2.7 \mathrm{Ga}(5,6)$. Nevertheless, it is arguable that the GOE itself is currently the strongest evidence for cyanobacterial oxygenic photosynthesis, but ongoing and future research will ultimately determine the robustness of the existing evidence for pre-GOE oxygenesis. However, if Archean organic biomarker research is to continue in the future, pockets of better preserved rocks must be identified using 
multiple screening techniques (e.g. Rock-Eval, H/C ratios, Raman spectroscopy, etc.). In the event that thermal maturity screening reveals Archean rocks with significantly lower thermal alteration, ideally within the oil window, then the techniques described in chapter two (drilling, sampling, storage, concentration gradients, HyPy, CSIA, etc.) should be used to minimize and assess hydrocarbon biomarker contamination.

One of the primary findings in chapter 3 was identifying a discrepancy between the bulk organic carbon isotopic excursion (CIE) and the compound specific CIE through the Toarcian oceanic anoxic event. The magnitude of the CIE needs to be accurately known in order to model possible sources of ${ }^{13} \mathrm{C}$-depleted carbon. Marine and terrestrial molecular isotopic records should be compiled in future studies of additional sites to evaluate whether the CIE is reproducibly smaller in compound specific records compared to bulk organic carbon isotopic records, which will allow further testing of the proposed source mixing model for the larger CIE recorded in bulk organic matter. If this hypothesis can be verified in future work, then less carbon may be required to explain the carbon cycle perturbation that occurred in the Early Jurassic.

The other significant finding of chapter 3 was discovering okenane in marine rocks younger than the Paleoproterozoic for the first time. Subsequent work (chapter 4) illustrated how pervasive okenane is in marine oils and rock extracts and challenges the traditional interpretation of this class of compound as only reflecting an autochthonous water column signature. The multiple environmental sources for the green and purple sulfur bacterial carotenoids will likely be difficult to disentangle, yet future studies focusing on transport of carotenoids from coastal and benthic sources will help to test the feasibility and ubiquity of this mechanism in modern and ancient systems. Continued efforts to identify green and purple sulfur bacteria and their pigments in transiently sulfidic systems in the modern ocean (e.g. chapter 5) will also test whether the application of these paleoredox proxies truly face a "no analog" problem rather than the case where these compounds have not been found in relevant systems because these 
systems have not been adequately examined for evidence. Another future challenge will be to distinguish cases where these compounds record a water column signature of a locally restricted paleobasin, as is the case for green and purple sulfur bacterial pigments in modern marine settings, rather than larger environmental conditions or perturbations. The improved interpretation of these compounds will significantly affect how we understand the redox chemistry of the oceans during the Proterozoic and Phanerozoic oceanic anoxic events.

\section{References}

1. Lyons TW, Reinhard CT, Planavsky NJ (2014) The rise of oxygen in Earth's early ocean and atmosphere. Nature 506:307-315.

2. Planavsky NJ et al. (2014) Evidence for oxygenic photosynthesis half a billion years before the Great Oxidation Event. Nat Geosci 7:283-286.

3. Anbar AD et al. (2007) A Whiff of Oxygen Before the Great Oxidation Event? Science 317:1903-1906.

4. Fischer WW et al. (2014), " Archean 'Whiffs of oxygen' tied to post-depositional processes" Goldschmidt abstracts, pp. 701.

5. Bosak T, Liang B, Sim MS, Petroff AP (2009) Morphological record of oxygenic photosynthesis in conical stromatolites. Proc Natl Acad Sci USA:1-14.

6. Bosak T, Knoll AH, Petroff AP (2013) The Meaning of Stromatolites. Annu Rev Earth Planet Sci 41:21-44. 


\section{Appendix A:}

\section{Methanogenic burst in the end-Permian carbon cycle}

This appendix was published in Proceedings of the National Academy of Sciences USA (PNAS) in 2014 and is reprinted here with their permission as granted in the original copyright agreement.

Rothman DH, Fournier GP, French KL, Alm EJ, Boyle EA, Cao C, Summons RE (2014) Methanogenic burst in the end-Permian carbon cycle. Proc Natl Acad Sci USA 111:54625467. 


\section{Methanogenic burst in the end-Permian carbon cycle}

Daniel H. Rothman ${ }^{a, b}, 1$, Gregory P. Fournier ${ }^{c}$, Katherine L. French ${ }^{b}$, Eric J. Alm ${ }^{c}$, Edward A. Boyle ${ }^{b}$, Changqun Cao ${ }^{d}$, and Roger E. Summons ${ }^{b}$

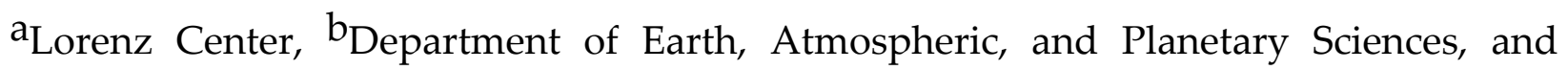
cDepartment of Biological Engineering, Massachusetts Institute of Technology, Cambridge, MA 02139; and d State Key Laboratory of Palaeobiology and Stratigraphy, Nanjing Institute of Geology and Palaeontology, Chinese Academy of Sciences, Nanjing 210008, China

ABSTRACT: The end-Permian extinction is associated with a mysterious disruption to Earth's carbon cycle. Here we identify causal mechanisms via three observations. First, we show that geochemical signals indicate superexponential growth of the marine inorganic carbon reservoir, coincident with the extinction and consistent with the expansion of a new microbial metabolic pathway. Second, we show that the efficient acetoclastic pathway in Methanosarcina emerged at a time statistically indistinguishable from the extinction. Finally, we show that nickel concentrations in South China sediments increased sharply at the extinction, probably as a consequence of massive Siberian volcanism, enabling a methanogenic expansion by removal of nickel limitation. Collectively, these results are consistent with the instigation of Earth's greatest mass extinction by a specific microbial innovation.

Significance Statement: The end-Permian extinction is the most severe biotic crisis in the fossil record. Its occurrence has been attributed to increased $\mathrm{CO} 2$ levels deriving 
from massive Siberian volcanism. However, such arguments have been difficult to justify quantitatively. We propose that the disruption of the carbon cycle resulted from the emergence of a new microbial metabolic pathway that enabled efficient conversion of marine organic carbon to methane. The methanogenic expansion was catalyzed by nickel associated with the volcanic event. We support this hypothesis with an analysis of carbon isotopic changes leading up to the extinction, phylogenetic analysis of methanogenic archaea, and measurements of nickel concentrations in South China sediments. Our results highlight the sensitivity of the Earth system to microbial evolution.

Keywords: methanogenesis, horizontal gene transfer, microbial evolution, biogeochemical dynamics 
Main Text: The greatest rate of taxonomic loss during the end-Permian extinction-the most severe in the fossil record (1)-occurs within 20,000 y, beginning about 252.28 million years ago (Ma) (2) at a time precisely coincident (2) with geochemical signals indicating a severe and equally rapid perturbation to Earth's carbon cycle (1-6). Although probably related, neither the cause of the extinction nor the origin of the change in the carbon cycle is known. One possible linkage derives from the observation that massive Siberian volcanism occurs at roughly the same time as the extinction $(7,8)$. However, quantitative estimates of direct volcanic outgassing are much too small to account for the changes in the carbon cycle (9). Secondary effects of Siberian volcanism, such as the combustion of huge deposits of coal (10) or other forms of organic carbon (11), are more attractive quantitatively but still difficult to reconcile with observed geochemical changes (1-6). Reports of marine anoxia in the Late Permian $(5,12,13)$ also indicate changes in the carbon cycle. Moreover, the notion that a disturbance of the carbon cycle plays a significant role as a "kill mechanism" derives considerable support from observations of physiological differences between species that survived the extinction and those that did not (14-16).

Here we relate the principal observations of end-Permian environmental changemassive volcanism and changes in marine $\mathrm{CO}_{2}$ and $\mathrm{O}_{2}$ levels - to the transfer of genetic material, from a cellulolytic bacterium to a methanogenic archaeon, that enabled efficient methanogenic degradation of organic carbon (17). Our analysis is constructed from three key observations. First, we show that the form of time-dependent changes in the carbon isotopic record indicates an instability within the carbon cycle that is inconsistent with volcanic combustion of organic sediments but consistent with the expansion of a new microbial metabolic pathway. Second, we identify this pathway with efficient acetoclastic methanogenesis and show that the age of the last common ancestor of Methanosarcina, the genus using this pathway, is consistent with the time of 
the extinction. Because methanogens are limited by nickel $(18,19)$, the third component of our study presents an analysis of nickel deposited in South China sediments. We find that nickel concentrations rose just before the extinction, presumably as a consequence of Siberian volcanism, providing a mechanism not only to enhance the methanogenic expansion and its perturbation to the carbon cycle but also to amplify the development of marine anoxia. Taken as a whole, these results reconcile an array of apparently disparate observations about the end-Permian event.

\section{Growth of the Marine Carbon Reservoir}

Fig. 1A displays the carbon-isotopic record in Meishan, China (5). Immediately preceding the extinction event, the isotopic composition $\delta 1$ of carbonate carbon declines by about $7 \%$ o during a period of about 100 thousand years (Kyr), first slowly, and subsequently rapidly. At the same time, the isotopic composition $\delta 2$ of organic carbon also changes. We seek the physical fluxes in the carbon cycle that predict the chemical signals $\delta 1(t)$ and $\delta_{2}(t)$. Of particular interest is whether the apparent downward acceleration of the carbonate signal can provide a quantitative indication of the underlying biogeochemical dynamics.

To obtain such understanding, we consider the carbon cycle to be a simple exchange between globally mixed reservoirs of inorganic and organic carbon, via photosynthesis and respiration (20), and assume that the isotopic changes represent a perturbation from a preexisting steady state. We assume that the system is perturbed by an influx of isotopically light inorganic carbon of constant isotopic composition $\delta_{i}{ }^{\prime}<\delta 1$, forcing $\delta 1$

to decline out of its steady state. Because organic carbon is isotopically light, a decrease in its rate of burial is effectively indistinguishable from such light inputs. However, changes in organic burial can account for only a negligible fraction of the peak perturbed flux (SI Text). Changes in the burial flux of carbonate carbon are limited to an 
even smaller impact because its isotopic composition is identical to that of the inorganic reservoir. We therefore hold both burial rates constant to maintain simplicity. The mass $m_{1}(t)$ of the inorganic reservoir then grows with time $t$ in response to the increased inputs, by an amount $m_{1}{ }^{\prime}(t)=m_{1}-m_{1}{ }^{*}$, where $m_{1}{ }^{*}$ is the initial, steady-state, size of the reservoir. The normalized perturbation $M=m_{1}{ }^{\prime} / m_{1}{ }^{*}$ is then straightforwardly related to changes in the geochemical signals by (SI Text)

$$
h \frac{\mathrm{d} M}{\mathrm{~d} t}=\frac{\mathrm{d} \delta_{1}}{\mathrm{~d} t} M+F\left(\delta_{1}, \delta_{2}, \frac{\mathrm{d} \delta_{1}}{\mathrm{~d} t}\right)
$$

where $h=\delta_{i}{ }^{\prime}-\delta 1(t)<0$ determines the scale of the perturbation and the "force" $F$ is a weighted sum of two nonsteady-state effects: the nonzero derivative $\mathrm{d} \delta 1=\mathrm{d} t$ and the departures of $\delta 1$ and $\delta 2$ from their unperturbed values. The solution of Eq. 1 for $M(t)$ provides the normalized time-dependent perturbation of the marine reservoir of dissolved inorganic carbon (DIC). The way in which the DIC reservoir grows with time is a function of how it is forced out of its steady state; consequently, knowledge of $M(t)$ can be used to test models of the end-Permian carbon cycle.

The form of Eq. 1 provides an immediate clue. Because $\delta 1(t)$ accelerates sharply downward, $\mathrm{d} \delta 1=\mathrm{d} t$ is increasingly negative. Consideration of the unforced $(F=0)$ equation then suggests that $M$ grows exponentially or faster. Numerical solutions of Eq. 1 confirm this view. Fig. $1 \mathrm{~B}$ plots $M(t)$ for the case $\delta_{i}{ }^{\prime}=-28 \%$, representative of the isotopic composition of remineralized organic carbon, assuming a constant sediment accumulation rate between the known dates (2). The curvature of the log-linear plot in Fig. 1B, Inset suggests that the growth of $M(t)$ is faster than exponential. (Although the total quantity of light carbon required for the isotopic excursion depends on $\delta_{i}{ }^{\prime}(1,2,6$, 9), the shape of $M(t)$ is independent of $\delta_{i}{ }^{\prime}$ when, as here, $\delta_{i}{ }^{\prime}$ is much smaller than $\delta_{1}$.) 
Superexponential growth of the marine inorganic carbon reservoir implies that the carbon cycle behaves nonlinearly. As we show later, a simple dynamical mechanism containing a leading-order nonlinearity predicts superexponential growth proportional to $\left(t_{\mathcal{C}}-t\right)^{-1}$. Such a growth law, where $t_{\mathcal{C}}$ is coincident with the extinction peak, is given by the red-dashed line in Fig. 1B. The burst near $t_{C}-$ an incipient singular blow-up-can be traced back to the rapid downward acceleration of the carbonate signal, a feature that is not exhibited by the linearly decreasing isotopic excursions of the Early Triassic (21).

Fig. 2 combines this analysis with the analogous carbon isotopic signal in the Gartnerkofel-I core drilled in the Carnic Alps, Austria $(3,4)$. Cyclostratigraphic analysis of the Gartnerkofel core indicates that the sediment accumulation rate over the corresponding interval is approximately constant (22). When the accumulation rate is set to $22.5 \mathrm{~cm} / \mathrm{Kyr}$, approximately within a factor of 2 of the earlier estimate (22), reconstructions of $M(t)$ for Gartnerkofel are qualitatively similar to that for Meishan. Fig. 2, which superposes both reconstructions in linear, log-linear, and log-log plots, confirms the inferences already drawn from the Meishan data. Moreover, the similarity of the Meishan and Gartnerkofel reconstructions validates our assumption of a constant accumulation rate at Meishan. We conclude that $M(t)$ grows no slower than exponentially, and likely superexponentially.

These observations impose constraints on interpretations of end-Permian environmental change. For example, $\mathrm{CO}_{2}$ released to the atmosphere from a single, massive Siberian coal-basalt eruption (10) would be mostly transferred to the oceans after about $10^{2}-10^{3}$ y (23). The uptake rate would decrease with time as the oceans acidify, reducing their capacity to take up more $\mathrm{CO}(23) . M(t)$ would then grow sublinearly, qualitatively different from what is shown in Figs. 1 and 2. Alternatively, 
such an eruption could be more gradual, limited by the rate at which vents form to release overpressured gas arising from contact metamorphism in intruded sills (11). The pressure released by each vent decreases overpressurization. Wherever the pressure is lowered, the rate of vent formation would decrease, and $M(t)$ would again be sublinear. It is possible, however, that vents were sufficiently dispersed in space so that their formation times were essentially independent and randomly distributed over some (possibly small) interval of time; in this case, one expects a roughly constant rate of $\mathrm{CO}_{2}$ emission, and $M(t)$ would grow linearly. Likewise, the release of methane from methane hydrates $(1,9)$ could be clustered in time, but there is no reason to expect total methane emissions to grow much faster than linearly, in part because any resulting warming - a potential positive feedback - would be only logarithmically sensitive to increased $\mathrm{CH}_{4}$ and $\mathrm{CO}_{2}$ levels (24). Each of these scenarios would produce geochemical signals that qualitatively differ from those predicted by exponential or superexponential growth (SI Text). The constraints provided by this reasoning derive only from the shape of $M(t)$, not the quantity of isotopically light carbon required for the event.

\section{The Carbon Source and Its Remobilization}

We seek a mechanism that can result in growth of $M(t)$ that is exponential or faster. Because such an upheaval of the carbon cycle implies substantial changes within the microbial biosphere, we hypothesize that the perturbation arises from the emergence of a new regime of microbial metabolic activity. We show below how such a mechanism leads to the observed dynamics. Before doing so, we first identify two important ingredients of our hypothesis: a suitably large source of degradable organic carbon and the new metabolic pathway that would be favored for its consumption.

In terms of the modern carbon cycle (25), our reconstructions of $M(t)$ require about 
7,000-14,000 Gt of remineralizable organic carbon. In the Late Permian, a low- $\mathrm{O}_{2}$ marine environment $(5,12,13)$ would have increased the concentration of organic matter in sediments. The high values of $\delta 1$ immediately before the perturbation suggest that organic carbon was sequestered in sediments at a rate at least $50 \%$ greater than usual (SI Text), which, in modern terms (25), corresponds to excess sequestration of at least $0.08 \mathrm{Gt} \mathrm{C} \mathrm{yr}^{-1}$. Integrated over the first $175 \mathrm{Kyr}$ of Fig. 1A, this corresponds to $14,000 \mathrm{Gt}$ C. We suggest that a substantial fraction remained remineralizable and relatively labile compared with organic carbon in modern sediments, thereby overcoming the apparent limitations (2) posed by the relatively small size of modern pools of isotopically light organic carbon (26) (e.g., methane hydrates, fossil fuels, soil organic carbon, and peat).

The accumulation of sedimentary organic matter would have been especially sensitive to changes in the biosphere's ability to metabolize the products of fermentation. Among these products, acetate provides a major growth substrate for methanogens. The conversion of acetate to methane by methanogenic archaea-acetoclastic methanogenesis (27) - begins by activating acetate to acetyl coenzyme A (acetyl-CoA). Carbon monoxide dehydrogenase (CODH) then catalyzes the cleavage of acetyl-CoA, after which, in common with the utilization of all methanogenic substrates, methylcoenzyme $\mathrm{M}$ reductase (MCR) catalyzes the reduction of a methyl group to methane (27). The activation to acetyl-CoA within methanogens occurs via two different pathways in two distinct groups of organisms: Members of the family Methanosaetaceae use a single-step acetyl-CoA synthase (ACS) pathway, whereas some members of the genus Methanosarcina use a two-step acetate kinase (AckA)-phosphoacetyl transferase (Pta) pathway (27). The AckA/Pta pathway is more energy efficient, requiring only one ATP molecule per acetate molecule activated, whereas the ACS pathway requires two (28). Growth on acetate using the low-efficiency ACS pathway within Methanosaeta is 
thermodynamically possible because of unique, poorly understood innovations in their electron transport chain (29); this limitation may be responsible for their observed slow rate of growth (30).

Fournier and Gogarten (17) have recently shown that the high-efficiency pathway in Methanosarcina evolved via a single horizontal gene transfer event, probably from a clade of cellulolytic bacteria belonging to the class Clostridia, after the mid-Ordovician evolution of vascular land plants (450-500 Ma). This is the only methanogenic pathway shown to have evolved via gene transfer. It also appears to be a conspicuously recent event within the evolution of methanogenesis, as all other methanogenic pathways have a broader phylogenetic distribution implying much more ancient origins. Methanosaeta may be more widespread in modern low-acetate marine environments (31). However, the dominance of the high-efficiency AckA-Pta pathway at high acetate concentrations (32) combined with the greater growth potential of Methanosarcina suggests that conditions for the emergence of acetoclastic Methanosarcina-specifically, a low- $\mathrm{O}_{2}$ marine environment $(5,12,13)$ and the accumulation of sedimentary organic matterwould have been favorable in the Late Permian. Moreover, reports of significantly reduced marine sulfate concentrations (33-36) suggest that competition from sulfatereducing bacteria would have been diminished, thereby amplifying the importance of methanogenesis in Late Permian marine sediments.

\section{Phylogenetic Analysis}

The relevance of acetoclastic Methanosarcina to the end-Permian event depends crucially on the timing for the ancestor of this group. To obtain an estimate for this date, we reconstructed archaeal phylogenies from 50 representative genomes and constructed relaxed molecular clock chronograms using PhyloBayes 2.3 (SI Text). Fig. 3A illustrates our results. To estimate the time $\tau_{\mathrm{m}}$ of the last common ancestor of known acetoclastic 
representatives of Methanosarcina, we generated chronograms using four independent ribosome-based datasets, separately containing 29 concatenated universally conserved ribosomal proteins, 12 concatenated archaeal-specific ribosomal proteins, 16S ribosomal RNA, and $23 S$ ribosomal RNA. The phylogeny of these ribosomal components reflects the vertical cellular history of the Methanosarcinales, including Methanosarcina and its descendants, the recipient lineage of the ackA/pta transfer. We further assume that ribosomal sequences evolve in a relatively clock-like manner, providing more reliable dates than most other genes in the absence of internal calibration. Our four independent age estimates, shown by the gray bars in Fig. 3A, are consistent with each other. Combining them together yields the joint estimate $\tau_{m}=240 \pm 41$ Ma depicted by the black bar in Fig. 3A, strikingly close to the end-Permian extinction (SI Text). The discrepancy with a previous estimate (37) derives from the earlier use of an autocorrelated clock model that is less reliable for the estimation of deep-time phylogenies than the approach used here (SI Text).

Additional phylogenetic character analysis of $16 S$ sequences from 33 species within the genus Methanosarcina lends increased precision to the placement of the gene transfer event within the chronogram of Fig. 3A. As shown in Fig. 3B, all Methanosarcina strains that grow readily on acetate diverge within the M. acetivorans/M. mazei/M. barkeri clade, whereas all strains shown not to grow on acetate diverge more deeply within the tree. This result supports a relatively recent horizontal transfer of ackA/pta within the Methanosarcina genus (SI Text), at a time consistent with the Late Permian. The combined results of Fig. 3 therefore support the hypothesis that the emergence of the acetoclastic pathway in Methanosarcina provided the microbial instigation of the endPermian burst in the carbon cycle.

\section{Methanogenic Expansion}


Wherever sulfate was limiting-because of a widespread drawdown of sulfate levels (33-36), a localized depletion by sulfate reducers, or both - the introduction of the highefficiency acetoclastic pathway would have diminished a thermodynamic barrier (38) to greater acetate production by fermenters. Not only would acetate be converted more quickly to methane, but also sedimentary organic matter would be fermented more rapidly to acetate. Methanosaeta would have played a supporting role, but a preexisting steady state excludes the possibility that Methanosaeta itself would have excited the perturbation.

The resulting methane burst would have been oxidized to $\mathrm{CO} 2$, either by anaerobic methanotrophs at the expense of any remaining sulfate or aerobically. $\mathrm{O}_{2}$ levels, which were likely already low $(5,12,13)$, would have been depressed further. Given the assumptions of an effectively unlimited substrate and a preexisting steady state, the size of the acetoclastic methanogenic niche-i.e., the carrying capacity $K$-would therefore have increased, at a rate proportional to the rate at which nearby sulfate and $\mathrm{O}_{2}$ were depleted. Taking the depletion rate proportional to the methane flux, we then have $\mathrm{d} K / \mathrm{d} t=k 1 \mathrm{~d} A / \mathrm{d} t$, where $A \propto M$ is the total methane production and $k 1$ is a conversion constant. Integrating both rates yields

$$
K(t)=k_{0}+k_{1} A(t)
$$

where $k_{0}$ is the initial (unperturbed) carrying capacity. At the time scale of the geochemical signals $\left(>10^{3} \mathrm{y}\right)$, the methanogenic population is always at carrying capacity. The methane production rate is therefore

$$
\mathrm{d} A / \mathrm{d} t=\beta K,
$$

where $\beta$ is the individual metabolic rate. Eqs. 2 and 3 describe unstable growth: 
Increasing methane production $(A)$ increases the carrying capacity $(K)$, which in turn increases the methane production rate $\mathrm{d} A=\mathrm{d} t$. Then if $\beta$ is constant, $A(t)$ grows exponentially.

However, several mechanisms may have acted to increase $\beta$. These include the aforementioned interactions with other microbial communities; warming due to higher $\mathrm{CO}_{2}$ and methane levels; adaptive radiation (39) as subtaxa evolved to more efficiently use acetate in particular environments; and greater access to any limiting mineral nutrients as the anoxic, methanogenic niche rose upwards. Each of these mechanisms implies a functional dependence on the total methane production $A$. We hypothesize a linear response:

$$
\beta(t)=\beta_{0}+\beta_{1} A(t)
$$

where $\beta_{0}$ is the initial metabolic rate and $\beta_{1}$ is a constant. Inserting Eqs. 2 and 4 into Eq. 3, we obtain

$$
\mathrm{d} A / \mathrm{d} t=a_{0}+a_{1} A+a_{2} A^{2},
$$

where $a_{0}=\beta_{0} k_{0}, a_{1}=\beta_{1} k_{0}+\beta_{0} k_{1}$, and $a_{2}=\beta_{1} k_{1}$. For nonzero $a 2$, the solution $A(t)$ blows up at a time $t_{\mathcal{C}}$ determined by initial conditions. Near $t_{\mathcal{C}}$, the dynamics are dominated by the nonlinearity. To leading order,

$$
A(t)=\frac{1}{a_{2}\left(t_{c}-t\right)}, \quad t \rightarrow t_{c},
$$

which is drawn as the dashed red curve in Fig. 1B and the dashed straight line in Fig. 2C (as $M \propto A$, using the best-fitting scale factor). The good fit supports the nonlinear model (Eq. 5), but it raises an important question: As $t$ approached $t_{\mathcal{C}}-$ the time of peak extinction activity-how could the growth of the methanogenic community have been 
sustained?

\section{Nickel Limitation and the Meishan Nickel Record}

Methanogens require nickel (18). The active site of the enzyme MCR, used by all methanogens, is the nickel cofactor F430; moreover, the CODH enzyme complex used by acetoclastic methanogens also contains a nickel cofactor (40). Seawater concentrations of nickel have likely been beneath the limiting threshold for methanogens for roughly the last 2 billion years (19). Accordingly, we suggest that methanogenesis in Late Permian sediments was limited by nickel. If so, the methanogenic expansion would have required increased access to nickel.

Kaiho et al. (41) have reported a sharp increase in the concentration of nickel in Meishan sediments coincident with the carbon isotopic spike. Because their analysis extends only through the last $5 \%$ of the $7 \%$ carbon isotopic spike and does not consider the effects of lithologic changes, we have performed new measurements of nickel concentrations at Meishan, not only over the entire interval shown in Fig. 1 but also well above it, and have corrected our measurements for variable concentrations of carbonate (SI Text). Fig. 4 displays our results. Nickel concentrations before the carbon isotopic spike are typically at least twice as high as after, and are up to seven times greater just before the abrupt downturn. With such elevated concentrations, nickel would no longer limit methanogenic activity. All methanogens would have prospered, but the successful evolution and rapid expansion of acetoclastic Methanosarcina would have been especially favored in the substrate-rich end-Permian environment.

The nickel likely originated from Siberia. Earth's largest economic concentration of nickel is in the Noril'sk region, deposited during the emplacement of the Siberian traps (42). The Meishan nickel signal may therefore represent changes in ocean chemistry 
induced by the Siberian eruptions, thereby linking the growth of acetoclastic Methanosarcina to massive Siberian volcanism. The apparent insensitivity of nickel to redox processes (43-45) supports this interpretation. However, elemental redistribution during diagenetic redox cycling could represent an alternative cause of nickel enrichment in the sediment (46); further analysis of redox-sensitive elements will help evaluate that possibility.

\section{Conclusion}

Our principal observations-a superexponential burst in the carbon cycle, the emergence of efficient acetoclastic methanogenesis, and a spike in the availability of nickel-appear straightforwardly related to several features of end-Permian environmental change: Siberian volcanism $(7,8)$, marine anoxia $(5,12,13)$, and ocean acidification (14-16). A single horizontal gene transfer (17) instigated biogeochemical change, massive volcanism acted as a catalyst, and the resulting expansion of acetoclastic Methanosarcina acted to perturb $\mathrm{CO}_{2}$ and $\mathrm{O}_{2}$ levels. The ensuing biogeochemical disruption would likely have been widespread. For example, anaerobic methane oxidation may have increased sulfide levels (47), possibly resulting in a toxic release of hydrogen sulfide to the atmosphere, causing extinctions on land (48). Although such implications remain speculative, our work makes clear the exquisite sensitivity of the Earth system to the evolution of microbial life.

ACKNOWLEDGMENTS: We thank Sam Bowring for asking questions that inspired this work, Kevin Peterson for making suggestions that widened its scope, and Shuzhong Shen for leading a field trip to South China. We also thank F. Azam, T. Bosak, S. Burgess, T. Cronin, O. Devauchelle, L. Elkins-Tanton, D. Erwin, D. Fike, C. Follett, Y. Friedman, H. Hartman, K. Noll, J. Payne, A. Petroff, F. Rohwer, and E. Tsiperman for helpful discussions. This work was supported by the National Aeronautics and Space 
Administration Astrobiology Institute (NNA08CN84A and NNA13AA90A), the National Science Foundation (OCE-0930866, DEB-0936234, and DGE-1122374), the National Natural Science Foundation of China (41290260), and the National Basic Research Program of China (2011CB808905).

\section{References}

1. Erwin DH (2006) Extinction: How Life on Earth Nearly Ended 250 Million Years Ago (Princeton University Press, Princeton).

2. Shen SZ, et al. (2011) Calibrating the end-Permian mass extinction. Science 334(6061): 1367-1372.

3. Holser WT, et al. (1989) A unique geochemical record at the Permian/Triassic boundary. Nature 337:39-44.

4. Magaritz M, Holser WT (1991) The Permian-Triassic of the Gartnerkofel-1 core (Carnic Alps, Austria): Carbon and oxygen isotope variation. Abh Geol Bundesanst 45:149-163.

5. Cao C, et al. (2009) Biogeochemical evidence for euxinic oceans and ecological disturbance presaging the end-Permian mass extinction event. Earth Planet Sci Lett 281(3-4):188-201.

6. Korte C, Kozur HW (2010) Carbon-isotope stratigraphy across the Permian-Triassic boundary: A review. J Asian Earth Sci 39(4):215-235.

7. Renne PR, Basu AR (1991) Rapid eruption of the Siberian Traps flood basalts at the Permo-Triassic boundary. Science 253(5016):176-179.

8. Reichow MK, et al. (2009) The timing and extent of the eruption of the Siberian Traps large igneous province: Implications for the end-Permian environmental crisis. Earth Planet Sci Lett 277(1-2):9-20.

9. Berner RA (2002) Examination of hypotheses for the Permo-Triassic boundary ex tinction by carbon cycle modeling. Proc Natl Acad Sci USA 99(7):4172-4177.

10. Ogden DE, Sleep NH (2012) Explosive eruption of coal and basalt and the end Permian mass extinction. Proc Natl Acad Sci USA 109(1):59-62.

11. Svensen H, et al. (2009) Siberian gas venting and the end-Permian environmental crisis. 
Earth Planet Sci Lett 277(3-4):490-500.

12. Wignall PB, Twitchett RJ (1996) Oceanic anoxia and the end Permian mass extinction. Science 272(5265):1155-1158.

13. Isozaki Y (1997) Permo-Triassic boundary superanoxia and stratified superocean: Records from lost deep sea. Science 276(5310):235-238.

14. Knoll AH, Bambach RK, Canfield DE, Grotzinger JP (1996) Comparative Earth history and Late Permian mass extinction. Science 273(5274):452-457.

15. Knoll AH, Bambach RK, Payne JL, Pruss S, Fischer WW (2007) Paleophysiology and end-Permian mass extinction. Earth Planet Sci Lett 256(3-4):295-313.

16. Payne JL, Clapham ME (2012) End-Permian mass extinction in the oceans: An ancient analog for the twenty-first century? Annu Rev Earth Planet Sci 40:89-111.

17. Fournier GP, Gogarten JP (2008) Evolution of acetoclastic methanogenesis in Methanosarcina via horizontal gene transfer from cellulolytic Clostridia. J Bacteriol 190(3):1124-1127.

18. Diekert G, Konheiser U, Piechulla K, Thauer RK (1981) Nickel requirement and factor F430 content of methanogenic bacteria. J Bacteriol 148(2):459-464.

19. Konhauser KO, et al. (2009) Oceanic nickel depletion and a methanogen famine before the Great Oxidation Event. Nature 458(7239):750-753.

20. Rothman DH, Hayes JM, Summons RE (2003) Dynamics of the Neoproterozoic carbon cycle. Proc Natl Acad Sci USA 100(14):8124-8129.

21. Payne JL, et al. (2004) Large perturbations of the carbon cycle during recovery from the end-Permian extinction. Science 305(5683):506-509.

22. Rampino MR, Prokoph A, Adler A (2000) Tempo of the end-Permian event: High resolution cyclostratigraphy at the Permian-Triassic boundary. Geology 28(7):643646.

23. Archer D (2009) The Long Thaw (Princeton University Press, Princeton).

24. Pierrehumbert RT (2010) Principles of Planetary Climate (Cambridge University Press, Cambridge).

25. Hedges JI, Keil RG (1995) Sedimentary organic matter preservation: An assessment and speculative synthesis. Mar Chem 49(2-3):81-115.

26. Milkov A (2004) Global estimates of hydrate-bound gas in marine sediments: How much is really out there? Earth Sci Rev 66(3-4):183-197.

27. Ferry JG (1992) Methane from acetate. J Bacteriol 174(17):5489-5495. 
28. Berger S, Welte C, Deppenmeier U (2012) Acetate activation in Methanosaeta thermophila: Characterization of the key enzymes pyrophosphatase and acetyl-CoA synthetase. Archaea 2012:315153.

29. Welte C, Deppenmeier U (2011) Membrane-bound electron transport in Methanosaeta thermophila. J Bacteriol 193(11):2868-2870.

30. Barber RD, et al. (2011) Complete genome sequence of Methanosaeta concilii, a specialist in aceticlastic methanogenesis. J Bacteriol 193(14):3668-3669.

31. Mori K, Iino T, Suzuki KI, Yamaguchi K, Kamagata Y (2012) Aceticlastic and NaClrequiring methanogen "Methanosaeta pelagica" sp. nov., isolated from marine tidal flat sediment. Appl Environ Microbiol 78(9):3416-3423.

32. Min H, Zinder SH (1989) Kinetics of acetate utilization by two thermophilic acetotrophic methanogens: Methanosarcina sp. strain cals-1 and Methanothrix sp. straincals-1. Appl Environ Microbiol 55(2):488-491.

33. Horita J, Zimmermann H, Holland HD (2002) Chemical evolution of seawater during the Phanerozoic: Implications from the record of marine evaporites. Geochim Cosmochim Acta 66(21):3733-3756.

34. Newton RJ, Pevitt EL, Wignall PB, Bottrell SH (2004) Large shifts in the isotopic composition of seawater sulphate across the Permo-Triassic boundary in northern Italy. Earth Planet Sci Lett 218(3-4):331-345.

35. Bottrell SH, Newton RJ (2006) Reconstruction of changes in global sulfur cycling from marine sulfate isotopes. Earth Sci Rev 75(1-4):59-83. 36. Luo G, et al. (2010) Isotopic evidence for an anomalously low oceanic sulfate concentration following end-Permian mass extinction. Earth Planet Sci Lett 300(1-2):101-111.

37. Blank CE (2009) Not so old Archaea the antiquity of biogeochemical processes in the archaeal domain of life. Geobiology 7(5):495-514.

38. Fenchel T, Finlay BJ (1995) Ecology and Evolution in Anoxic Worlds (Oxford University Press, New York).

39. Schluter D (2000) The Ecology of Adaptive Radiation (Oxford University Press, New York).

40. Ragsdale SW (2009) Nickel-based enzyme systems. J Biol Chem 284(28):18571-18575.

41. Kaiho K, et al. (2001) End-Permian catastrophe by a bolide impact: Evidence of a gigantic release of sulfur from the mantle. Geology 29(9):815-818.

42. Naldrett AJ (2004) Magmatic Sulfide Deposits: Geology, Geochemistry and Exploration 
(Springer, New York).

43. Jacobs L, Emerson S, Huested SS (1987) Trace metal geochemistry in the Cariaco Trench. Deep-Sea Res Part A 34(5-6):965-981.

44. Haraldsson C, Westerlund S (1988) Trace metals in the water columns of the Black Sea and Framvaren Fjord. Mar Chem 23(3-4):417-424.

45. Lewis BL, Landing WM (1992) The investigation of dissolved and suspended-particulate trace metal fractionation in the Black Sea. Mar Chem 40:105-141.

46. Froelich PN, et al. (1979) Early oxidation of organic-matter in pelagic sediments of the eastern equatorial Atlantic: Suboxic diagenesis. Geochim Cosmochim Acta 43(7):10751090 .

47. Kaiho K, et al. (2012) Changes in depth-transect redox conditions spanning the endPermian mass extinction and their impact on the marine extinction: Evidence frombiomarkers and sulfur isotopes. Global Planet Change 94-95:20-32.

48. Kump LR, Pavlov A, Arthur MA (2005) Massive release of hydrogen sulfide to the surface ocean and atmosphere during intervals of oceanic anoxia. Geology 33(5):397-400. 

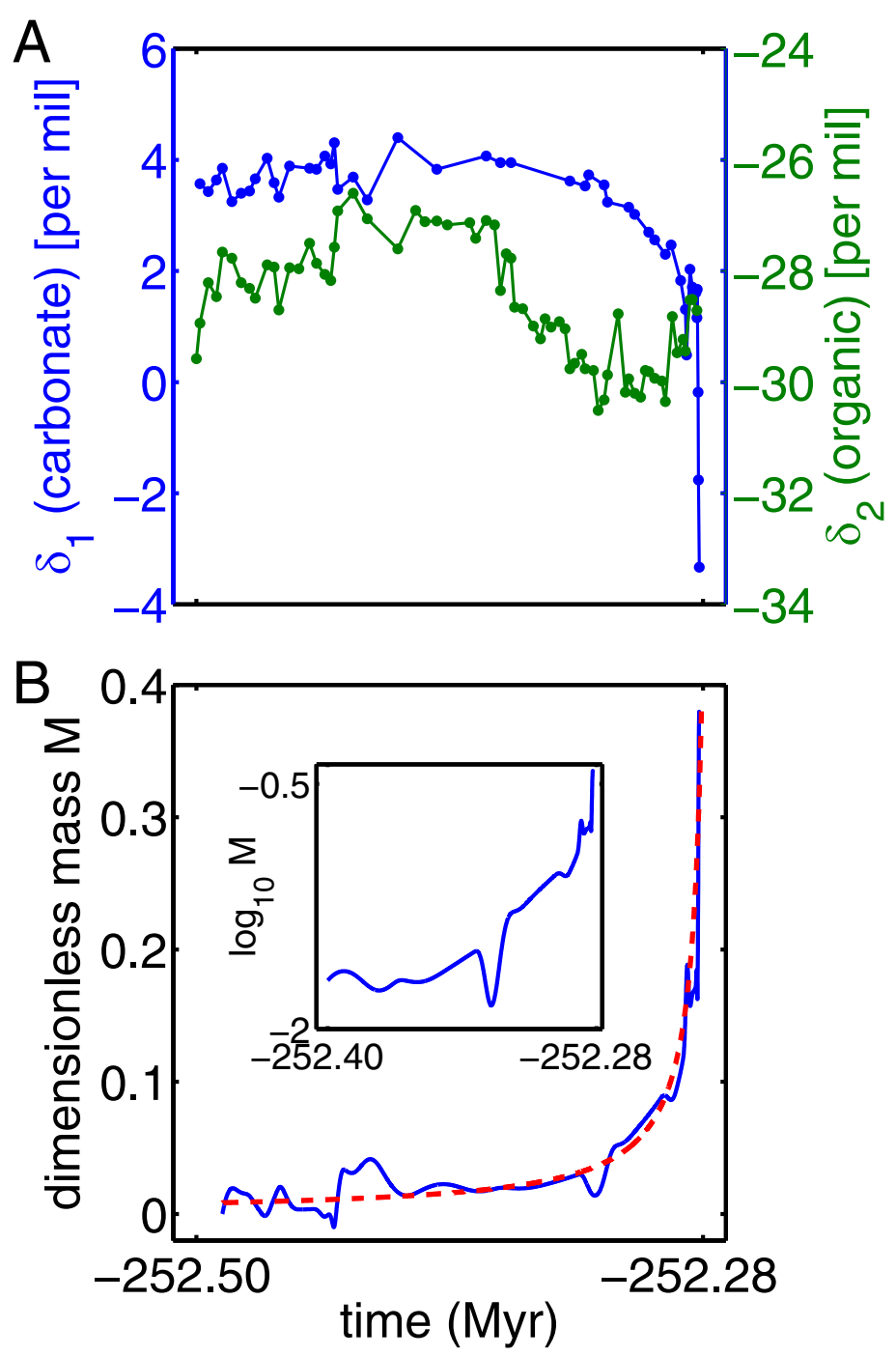

Fig. 1. Carbon isotopic signals immediately preceding the end-Permian extinction in Meishan, China, indicate superexponential growth of the marine inorganic carbon reservoir. (A) Inorganic ( $\delta 1$, blue) and organic ( $\delta 2$, green) carbon isotopic changes (5). The dates at both ends of the time axis are resolved within $\pm 0: 08 \mathrm{Myr}(2)$; in between, time is interpolated linearly in proportion to stratigraphic height. The peak extinction activity occurs at approximately -252.28 Myr (2). (B) Reconstruction (solid blue line) of the dimensionless mass $M(t)$ added to the marine inorganic carbon reservoir, assuming initial condition $M=0$ and an influx of isotopically light carbon with isotopic composition $\delta^{\prime} i=-28 \%$. (Inset) The same curve plotted on log-linear axes; the concave upward curvature suggests that $M(t)$ grows superexponentially. The dashed red line compares $M(t)$ to a superexponential growth law proportional to $\left(t_{c}-t\right)^{-1}$, where the critical time $t c$ is coincident with the extinction peak. 


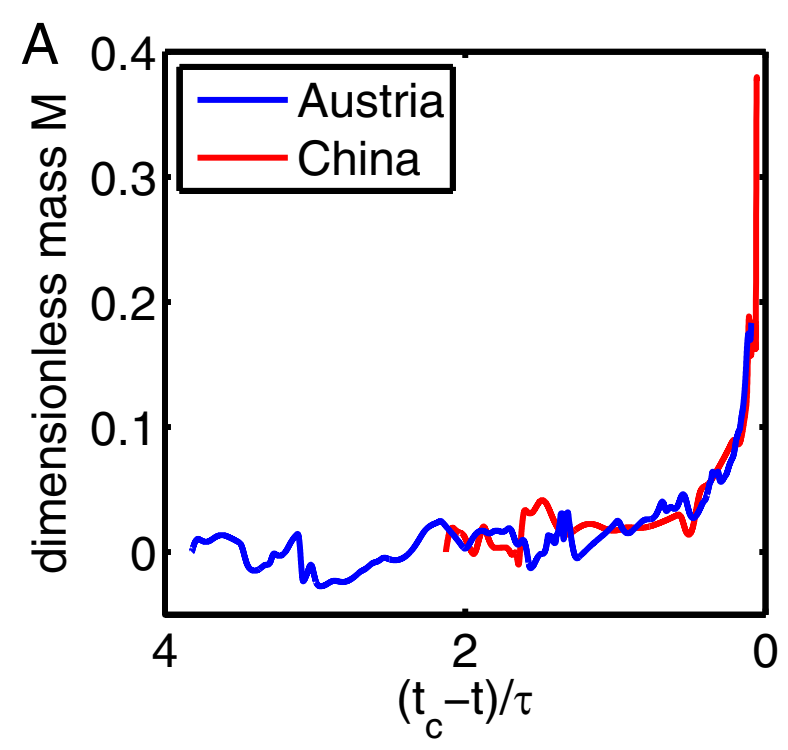

Fig. 2. Rescaled reconstructions of the mass $M(t)$ of the marine inorganic carbon reservoir for both the Meishan (red) and Gartnerkofel (blue) data, as a function of rescaled time $\left(t_{C}-t\right) / \tau$, where $\tau=100 \mathrm{Kyr}$ and time advances to the right. (A) Plots of $M$ vs. $\left(t_{c}-t\right) / \tau$ show that both curves behave similarly with respect to a critical time $t_{c}$. (B) Plots of $M(t)$ on log-linear axes suggest that both curves grow superexponentially. (C) Plots of $M(t)$ on $\log -\log$ axes compare well to a straight line (dashed black) with slope -1 ,

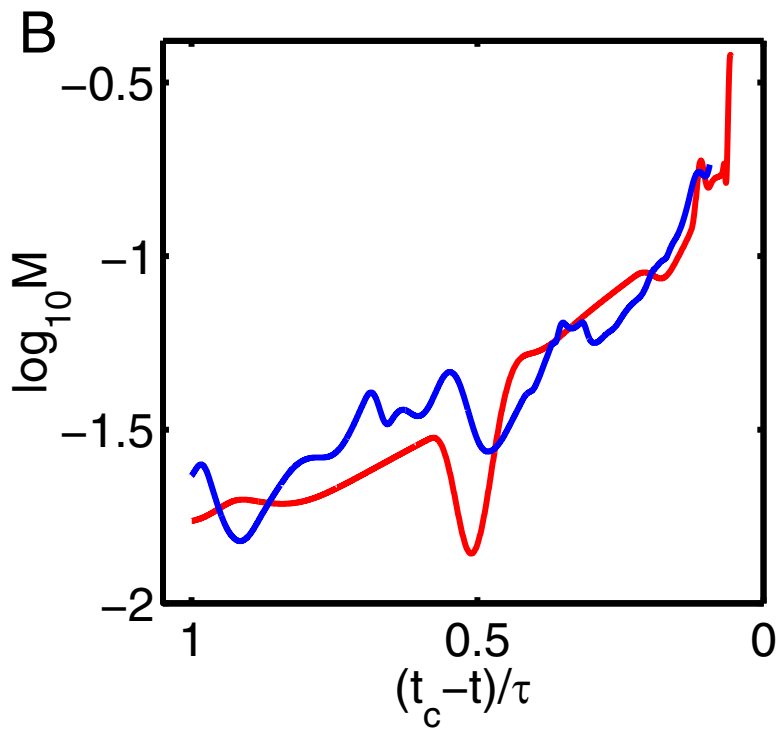
suggesting that both reconstructions grow like $\left(t_{c}-t\right)^{-1}$.

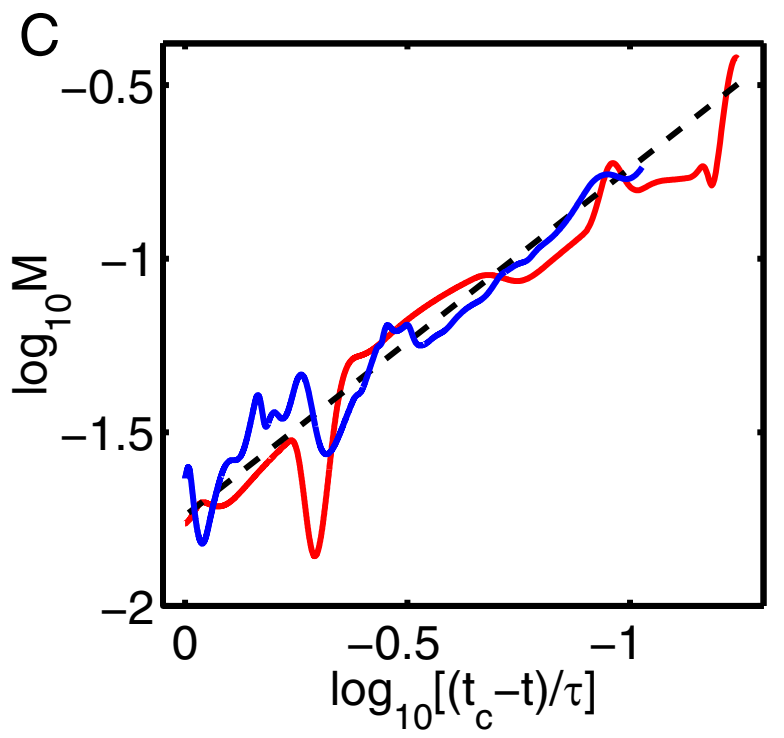



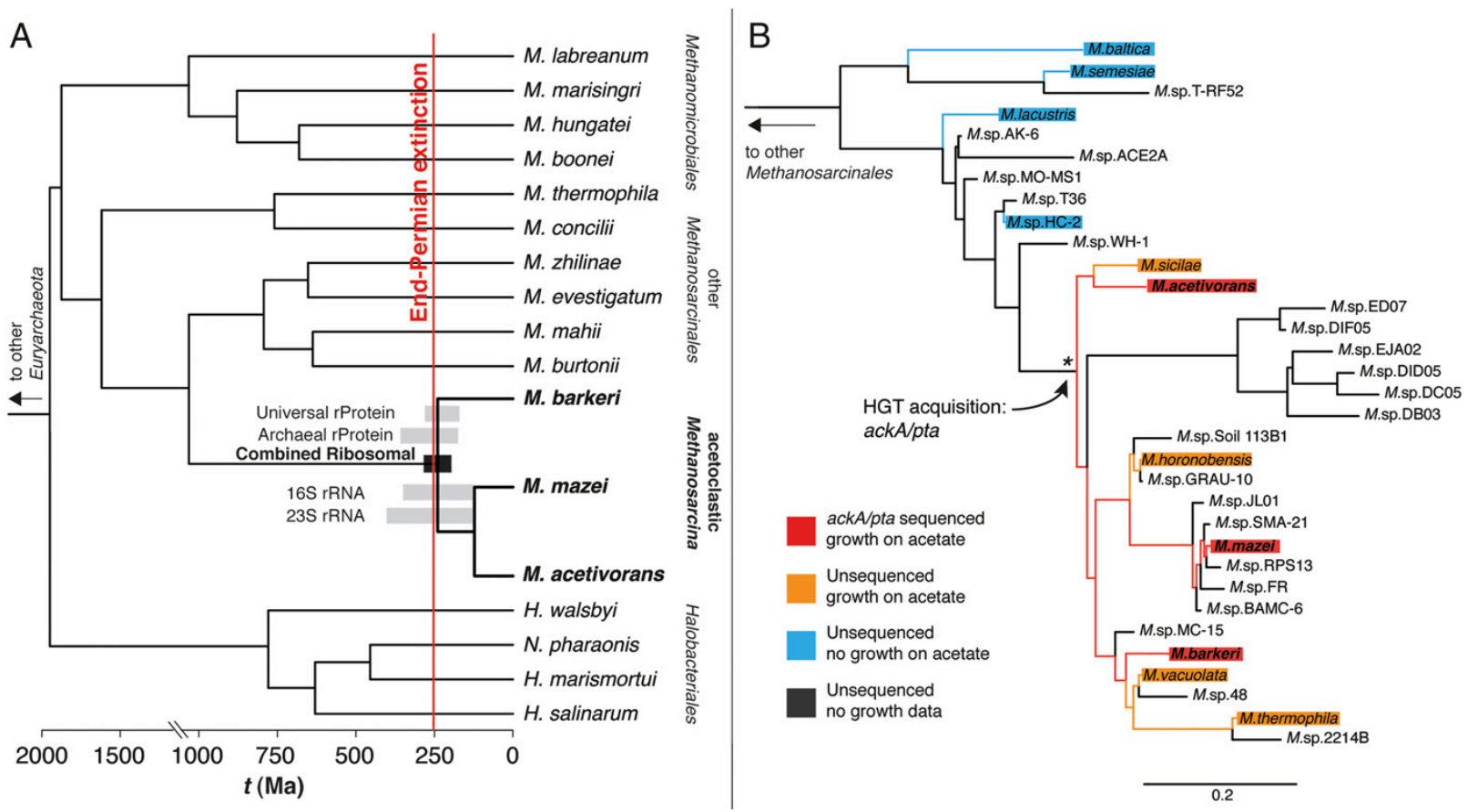

Fig. 3. (A) Subtree of calibrated archaeal chronogram showing Methanosarcinales and related groups. Acetoclastic Methanosarcina (bold) groups with other members of Methanosarcinales, including the distantly related acetoclastic Methanosaetaceae ( $M$. thermophila, M. concilii). Shaded bars indicate the estimated acetoclastic Methanosarcina ancestor age ranges $( \pm 1 \mathrm{SD})$ for each dataset. The combined estimate of $240 \pm 41 \mathrm{Myr}$ is indicated by the black bar. The tree was generated from the Universal rProtein dataset, with the age of acetoclastic Methanosarcina adjusted to match the combined estimate. (B) Members of the genus Methanosarcina shown to grow on acetate all descend from the ancestor of sequenced clade representatives containing the transferred ackA/pta genes $\left(^{*}\right)$, congruent with the age-estimated node in A. The relationships between known taxa are represented by their $16 \mathrm{~S}$ phylogenetic tree. 

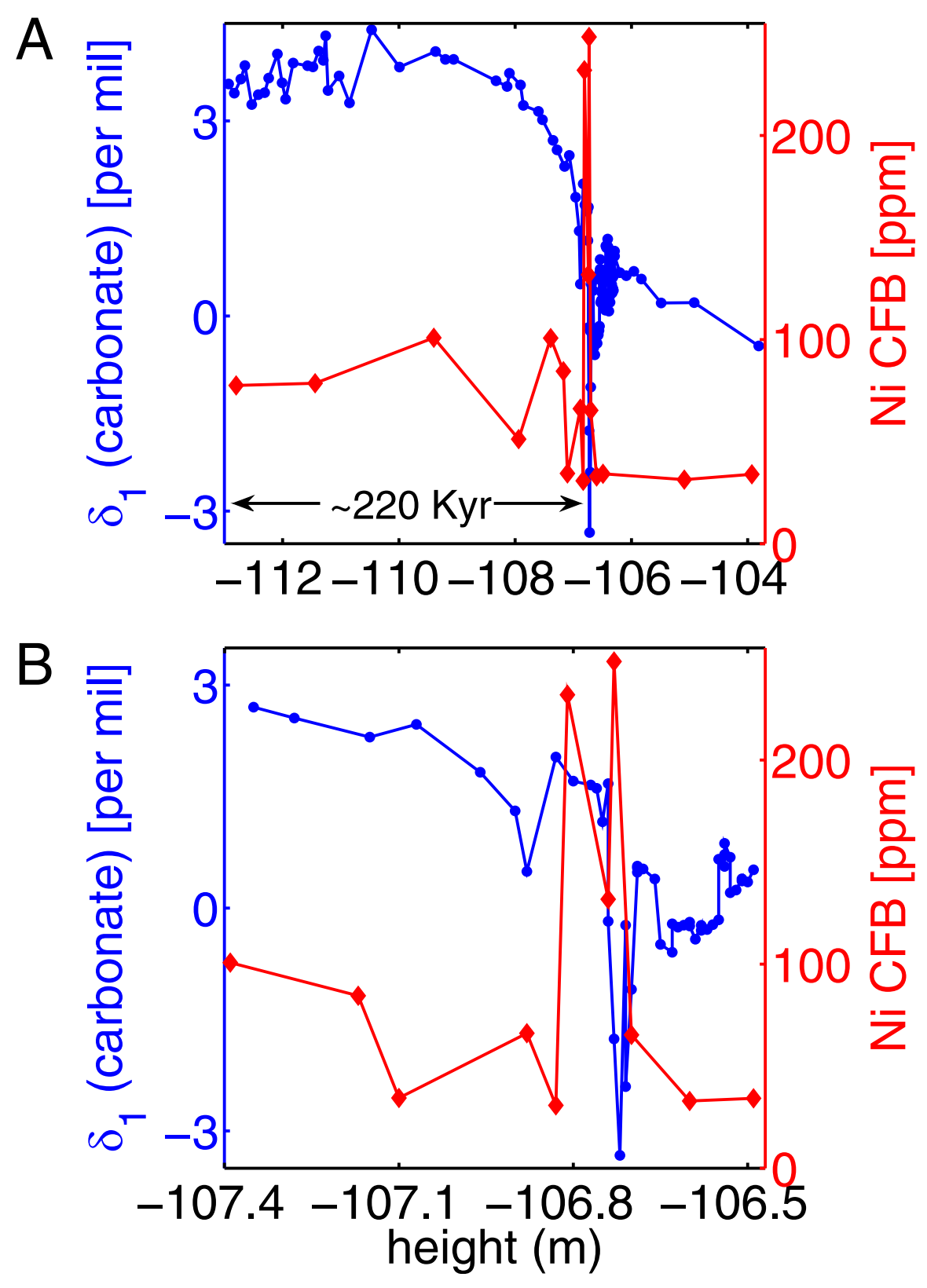

Fig. 4. Nickel concentrations (red) in the Meishan sediments, compared with the isotopic composition $\delta 1$ of carbonate carbon (blue). (A) Approximately $9 \mathrm{~m}$ of sedimentary section. (B) Close-up of the changes, over a range of less than $1 \mathrm{~m}$. The largest nickel concentration precedes the smallest value of $\delta 1$. Nickel concentrations are reported in terms of a carbonate-free basis (SI Text). 


\section{Supporting Information}

\section{Isotopic Dynamics}

Eq. 1 is derived from the model of ref. 1. As shown in Fig. S1, this model consists of two reservoirs of carbon, inorganic and organic. The isotopic compositions $\delta$ (inorganic carbon) and $\delta$ (organic carbon) change as

$$
\begin{aligned}
& \frac{\mathrm{d} \delta_{1}}{\mathrm{~d} t}=\frac{j_{i}}{m_{1}}\left(\delta_{i}-\delta_{1}\right)+\frac{j_{21}}{m_{1}}\left(\delta_{2}-\delta_{1}\right)+\frac{j_{12}}{m_{1}} \varepsilon \\
& \frac{\mathrm{d} \delta_{2}}{\mathrm{~d} t}=\frac{j_{12}}{m_{2}}\left(\delta_{1}-\varepsilon-\delta_{2}\right) \\
& \frac{\mathrm{d} m_{1}}{\mathrm{~d} t}=j_{i}-j_{12}+j_{21}-b_{1} \\
& \frac{\mathrm{d} m_{2}}{\mathrm{~d} t}=j_{12}-j_{21}-b_{2} .
\end{aligned}
$$

Here $j 12$ is the flux of photosynthetic production from reservoir $1 \rightarrow 2, j 21$ is the respiration or remineralization flux from reservoir $2 \rightarrow 1$, and $j i$ is the flux of external inorganic inputs, such as those resulting from weathering or volcanism, with isotopic com- position $\delta i$. The reservoir sizes $m 1$ and $m 2$ are, in general, time dependent, and lose mass by burial at rates $b_{1}$ and $b_{2}$. The transfer of carbon from the inorganic to the organic pool occurs with isotopic fractionation $\varepsilon$.

1.1 Response to Changing Inputs. We first focus on changes in the input flux $j i$ and the isotopic composition $\delta i$ that it carries. We assume that the turnover time of organic carbon, $m 2 / j 12$, is short compared with the time scale over which the isotopic composition of inorganic carbon changes. Then at time scales $\gg m_{1} / j i, \delta 2$ adjusts quasi-statically to changes in $\delta 1$ and/or $\varepsilon$ so that 


$$
\varepsilon=\delta_{1}-\delta_{2}
$$

The right-hand-side of Eq. S2 then vanishes, and we can substitute Eq. S5 into Eq. S1 to obtain

$$
\frac{\mathrm{d} \delta_{1}}{\mathrm{~d} t}=\frac{j_{i}}{m_{1}}\left(-\delta_{1}+\delta_{i}+\frac{j_{12}-j_{21}}{j_{i}} \varepsilon\right)
$$

We next assume that the difference between the production and respiration fluxes is balanced by the burial flux 62 of organic carbon, and that the latter is constant; i.e.,

$$
b_{2}=j_{12}-j_{21}=\text { const. }
$$

Eq. S1 then becomes

$$
m_{1} \frac{\mathrm{d} \delta_{1}}{\mathrm{~d} t}=j_{i}\left(\delta_{i}-\delta_{1}\right)+b_{2} \varepsilon
$$

Note that in steady state, the left-hand side vanishes, and we obtain the well-known relation $\delta 1=\delta i+f \varepsilon$, where $f=b 2 / j i$ is the organic burial fraction (2).

We consider perturbations from a steady state characterized by the isotopic compositions $\delta_{1}^{*}$ and $\delta_{i}^{*}$, the fractionation $\varepsilon^{*}$, the input flux $j_{i}{ }^{*}$, and the reservoir size $m_{1}{ }^{*}$. We suppose that the steady state is perturbed by an anomalous input flux $j_{i}{ }^{\prime}$ that carries an anomalous isotopic composition $\delta_{i}{ }^{\prime}$. Then the new input flux is given by

$$
j_{i}=j_{i}^{*}+j_{i}^{\prime}
$$

and the isotopic flow $j i \delta_{i}$ is similarly partitioned so that

$$
j_{i} \delta_{i}=j_{i}^{*} \delta_{i}^{*}+j_{i}^{\prime} \delta_{i}^{\prime}
$$


Substitution of Eqs. S9 and S10 into Eq. S8 gives

$$
m_{1} \frac{\mathrm{d} \delta_{1}}{\mathrm{~d} t}=j_{i}^{*}\left(\delta_{i}^{*}-\delta_{1}\right)+j_{i}^{\prime}\left(\delta_{i}^{\prime}-\delta_{1}\right)+b_{2} \varepsilon .
$$

In the preexisting steady state, $j_{i}{ }^{\prime}=0$, the left-hand side above vanishes, and we have the steady-state mass balance

$$
0=j_{i}^{*}\left(\delta_{i}^{*}-\delta_{1}^{*}\right)+b_{2} \varepsilon^{*} .
$$

Subtracting Eq. S12 from Eq. S11 and dividing by $j_{i}^{*}$, we obtain

$$
\frac{m_{1}}{j_{i}^{*}} \frac{\mathrm{d} \delta_{1}}{\mathrm{~d} t}=-\left(\delta_{1}-\delta_{1}^{*}\right)+\frac{j_{i}^{\prime}}{j_{i}^{*}}\left(\delta_{i}^{\prime}-\delta_{1}\right)+f\left(\varepsilon-\varepsilon^{*}\right),
$$

[S13]

where we have substituted $f=b 2 / j_{i}^{*}$. Rearranging Eq. S13, we obtain

$$
\frac{j_{i}^{\prime}}{j_{i}^{*}}=\frac{\left(m_{1} / j_{i}^{*}\right) \dot{\delta}_{1}+\delta_{1}-\delta_{1}^{*}-f\left(\varepsilon-\varepsilon^{*}\right)}{\delta_{i}^{\prime}-\delta_{1}},
$$

[S14]

where

$$
\dot{\delta}_{1}=\mathrm{d} \delta_{1} / \mathrm{d} t .
$$

We next decompose $m 1$ into its steady state $m 1^{*}$ and its perturbation $m 1^{\prime}(t)$ :

$$
m_{1}(t)=m_{1}^{*}+m_{1}^{\prime}(t) .
$$

Substituting Eq. S7 into S3, we find

$$
\frac{\mathrm{d} m_{1}}{\mathrm{~d} t}=j_{i}-b_{2}-b_{1}
$$

[S16]

We assume that the carbonate burial rate $b_{1}$ is constant. Then, subtracting $j_{i}^{*}=b_{1}+b_{2}$ 
from the right-hand side of Eq. S16 and substituting Eq. S15 for $m 1$, we find

$$
j_{i}^{\prime}=\frac{\mathrm{d} m_{1}^{\prime}}{\mathrm{d} t}
$$

[S17]

Inserting Eqs. S15 and S17 into Eq. S14, we obtain

$$
\frac{1}{j_{i}^{*}} \frac{\mathrm{d} m_{1}^{\prime}}{\mathrm{d} t}=\frac{\left[\left(m_{1}^{*}+m_{1}^{\prime}\right) / j_{i}^{*}\right] \dot{\delta}_{1}+\delta_{1}-\delta_{1}^{*}-f\left(\varepsilon-\varepsilon^{*}\right)}{\delta_{i}^{\prime}-\delta_{1}} .
$$

To simplify this expression, we first define the dimensionless mass

$$
M \equiv m_{1}^{\prime} / m_{1}^{*}
$$

and substitute it into Eq. S18 to obtain

$$
\tau \frac{\mathrm{d} M}{\mathrm{~d} t}=\frac{\tau(1+M) \dot{\delta}_{1}+\delta_{1}-\delta_{1}^{*}-f\left(\varepsilon-\varepsilon^{*}\right)}{\delta_{i}^{\prime}-\delta_{1}},
$$

where $\tau=m 1^{*} / j_{i}{ }^{*}$ is the steady-state turnover time of the inorganic reservoir with respect to $j_{i}^{*}$. We then define

$$
h=\delta_{i}^{\prime}-\delta_{1}(t)
$$

and

$$
F=\dot{\delta}_{1}+\tau^{-1}\left[\delta_{1}-\delta_{1}^{*}-f\left(\varepsilon-\varepsilon^{*}\right)\right] .
$$

Substitution into Eq. S20 then yields Eq. 1:

$$
h \frac{\mathrm{d} M}{\mathrm{~d} t}=\dot{\delta}_{1} M+F .
$$

1.2 Response to Changing Respiration Flux. We now consider an alternative scenario 
in which $j i$ remains constant but $j 21$ changes, such that

$$
j_{21}=j_{21}^{*}+j_{21}^{\prime}
$$

This case corresponds to the methanogenic perturbation. As a result of the drawdown of the organic reservoir, the mass $m 2$ is no longer considered constant.

We assume once again that $\delta 2$ adjusts rapidly to changes in $\varepsilon$ or $\delta 1$. Then, inserting Eqs. S5 and S24 into Eq. S1, we obtain

$$
m_{1} \frac{\mathrm{d} \delta_{1}}{\mathrm{~d} t}=j_{i}\left(\delta_{i}-\delta_{1}\right)+\left(j_{12}-j_{21}^{*}\right) \varepsilon-j_{21}^{\prime} \varepsilon
$$

We once again assume constant $b 2$, but we no longer assume the mass balance for $m 2$ implied by Eq. S7. We instead assume only that the production rate $j_{12}=j_{12}{ }^{*}=$ const. Then, after subtracting the steady solution Eq. S12 from Eq. S25, we obtain

$$
m_{1} \frac{\mathrm{d} \delta_{1}}{\mathrm{~d} t}=-j_{i}^{*}\left(\delta_{1}-\delta_{1}^{*}\right)-j_{21}^{\prime} \varepsilon+b_{2} \varepsilon
$$

where we have used $b 2=j 12^{*}-j 21^{*}$. Noting that $f=b 2 / j i^{*}$, we obtain, after rearrangement,

$$
\frac{j_{21}^{\prime}}{j_{i}^{*}}=\frac{\left(m_{1} / j_{i}^{*}\right) \dot{\delta}_{1}+\delta_{1}-\delta_{1}^{*}-f\left(\varepsilon-\varepsilon^{*}\right)}{-\varepsilon}
$$

Comparing this expression to Eq. S14, we find that Eq. S14 is equivalent to Eq. S27 when

$$
j_{i}^{\prime} \rightarrow j_{21}^{\prime} \quad \text { and } \quad \delta_{i}^{\prime} \rightarrow \delta_{2}
$$

where the latter expression derives from Eq. S5.

Consequently, Eq. S23 applies to changes in either $j i$ or $j 21$. In both cases, the burial rates $b_{1}$ and $b_{2}$ are fixed while $m 1$ and j21 can vary. However, because the role of the organic 
reservoir differs in the two cases, somewhat different assumptions underlie the model. These are summarized in Table S1.

1.3 Time-Varying Burial. In the preceding analysis, we have assumed constant burial fluxes. This assumption makes the analysis simpler, in part because we cannot distinguish a change in the burial flux from a change in the inputs. However, we can show that a change in the burial of organic carbon - which carries the necessary light isotopic composition-cannot account for the peak carbon flux at Meishan.

Suppose that the perturbation of the steady state consists entirely of a change of amount $b_{2}{ }^{\prime}$ to the organic burial flux $b_{2}$ while the input flux $j i=j_{i}{ }^{*}$. Then $b_{2}$ varies as

$$
b_{2}(t)=b_{2}^{*}+b_{2}^{\prime}(t),
$$

where $b_{2}{ }^{*}$ is the initial steady burial flux. Assuming, as in Response to Changing Inputs, that $m 2$ and $b_{1}$ are constant, we can subtract $j_{i}^{*}=b_{1}+b_{2}$ from Eq.S17 to obtain

$$
-b_{2}^{\prime}=\frac{\mathrm{d} m_{1}^{\prime}}{\mathrm{d} t} .
$$

Comparing this expression with Eq. S17, we find that changes in $j_{i}{ }^{\prime}$ are equivalent to changes in $b_{2}^{\prime}$, but with the opposite sign. Growth of $m 1$ therefore requires decreased burial. However, because burial cannot be negative, $m 1$ will grow only when

$$
-b_{2}^{*} \leq b_{2}^{\prime}<0 .
$$

We can compare the lower bound to our reconstruction by dividing both sides of Eq. S30 by $j_{i}^{*}$ and substituting Eq. S19 to obtain 


$$
\frac{-b_{2}^{\prime}}{j_{i}^{*}}=\tau \frac{\mathrm{d} M}{\mathrm{~d} t} .
$$

Our reconstructions of $M(t)$ suggest that the right-hand side-the dimensionless perturbed flux of isotopically light carbon-exceeded 40 when the end-Permian carbon isotopic excursion reached its minimum at Meishan. On the other hand, a preexisting steady state requires $b_{2}^{*}=j_{i}^{*}-b_{1}$ in addition to the lower bound of Eq. S31, constraining the left-hand side of Eq. S32 to be less than 1. Consequently, a drop in the organic burial flux can account for, at most, a negligible fraction of the peak carbon flux.

1.4 Numerical Reconstructions. Our reconstructions of $M(t)$ were performed by numerically integrating Eq. S23 with initial condition $M=0$, using the observations $\delta 1[t]$ and $\varepsilon(t)=\delta 1(t)-\delta 2(t)$ to construct the forcing function $\mathrm{F}(t)$ and the "scale" function $h(t)$. The isotopic composition of the perturbed flux was set to $\delta_{i}{ }^{\prime}=-28 \%$, and the preexisting, steady-state isotopic composition of carbonate carbon was set to $\delta 1^{*}=3.5 \%$. In the case of the Meishan dataset, which included $\varepsilon(t)$, we additionally assumed that the steady state was characterized by the burial fraction $f=0.3$ and $\varepsilon=31.67 \%$. These quantities are consistent with the pre-excursion data and collectively satisfy the steadystate mass balance relation (2)

$$
f=\frac{\delta_{1}^{*}-\delta_{i}^{*}}{\varepsilon^{*}},
$$

where $\delta_{1}^{*}=-6 \%$. The typical Phanerozoic value of $f$ is about $0.2(3)$; consequently the preexcursion organic burial fraction is about 50\% greater. For the Gartnerkofel data, which did not include $\varepsilon(t)$, we assumed $\varepsilon(t)=\varepsilon^{*}$. In both cases, the relaxation time scale of the carbon cycle was set to $\tau=100$ thousand years. Numerical integrations were performed using standard methods provided by Matlab. 
1.5 Graphical Comparison of Alternative Scenarios. Fig. S2 depicts four scenarios for the growth of $M(t)$ and the corresponding decline of $\delta 1(t)$. In Fig. S2A, the uppermost curve (a) represents sublinear growth of $M$. It is a solution of

$$
\frac{\mathrm{d} M}{\mathrm{~d} t}=J-M / \tau,
$$

where J represents a constant flux and $\tau$ is again the characteristic relaxation time. The remaining curves of Fig. S2A represent linear (b), exponential (c), and superexponential (d) growth; these curves are solutions of

$$
\frac{\mathrm{d} M}{\mathrm{~d} t} \propto M^{\beta},
$$

for $\beta=0,1$, and 2, respectively, The superexponential curve is the same as the red dashed curve of Fig. 1B and Eq. 6. Each of the remaining curves contains two parameters that are set by matching the initial and final values of $M(t)$ in the superexponential case. The corresponding curves for $\delta 1(t)$, obtained by solving Eq. S20 for $\delta 1(t)$ given $M(t)$ assuming $\varepsilon=\varepsilon^{*}$, are shown in Fig. S2B.

\section{Phylogenetic Analyses}

2.1 Phylogenetic Reconstructions. Protein-based phylogenies were generated from aligned, concatenated ribosomal protein datasets from 50 representative archaeal genomes as described and provided in refs. 4 and 5. For RNA-based chronogram analyses, phylogenies including these same taxa were generated from available $16 \mathrm{~S}$ and 235 sequences obtained from GenBankK (6), and aligned using the MUSCLE program (7). The phylogenetic tree for mapping acetate growth conditions was generated in the same way using all available $16 \mathrm{~S}$ sequences within Methanosarcina, rooted by $16 \mathrm{~S}$ sequences from other Methanosarcinales taxa. Trees were generated for each dataset 
using the program PHYML 3.0 (8), with the following parameters: substitution model $($ protein $)=$ WAG, substitution model $($ nucleotide $)=$ HKY, proportion of invariant sites $=$ estimated, rate categories $=4$, gamma shape parameter alpha $=$ estimated, tree topology search $=$ SPR. Trees were rooted between Euryarchaeota and Crenarchaeotal Thaumarchaeota/Korarchaeota groups.

2.2 Chronogram Calibrations. Chronograms were generated with the PhyloBayes 2.3 program (9), using uncorrelated gamma multipliers (-ugam) as a relaxed clock model. Nucleotide datasets were additionally analyzed using an autocorrelated Cox-IngersollRoss (CIR) process model (-cir). PhyloBayes permits ranged calibrations, outputs Gaussian distributions for estimated node ages, and is resistant to node-density artifacts (10). Results were compiled from converged runs $(\sim 3,000-5,000$ generations) with a $20 \%$ burn-in.

In the absence of direct fossil evidence to time the archaeal ancestor, an archaeal root calibration range of 3.5-3.9 billion years ago (Ga) was selected, consistent with most estimates $(11,12)$, reflecting a Late Hadean/Early Archaean origin of life and rapid diversification of major domains implicit in several models of early life evolution (1315). We are unaware of any seriously considered scenarios for a much later origin of the major domains. As our tree relies on a clock model in the absence of internal calibrations, the uncertainty in estimated divergence times arises from the prior distribution on the root. Models incorporating a lower-bound constraint of $2.7 \mathrm{Ga}$ for the ancestor of methanogens were also tested $(11,12)$. Although the biomarker used to impose this constraint has recently been shown to be unreliable, neither its inclusion nor removal significantly impact molecular clock estimates. In both cases, the estimated divergence time of methanogens was substantially older than $2.7 \mathrm{Ga}$, and therefore this calibration point was not "active" in its establishment, nor in the subsequent divergence times within methanogens, including the last common ancestor of acetoclastic 
Methanosarcina. To further validate this clock model, estimated divergence times within the methanogen clade were evaluated based on their temporal consistency with specific horizontal gene transfer (HGT) calibration events within the literature, analogous to existing validation techniques using fossil calibrations (16). Gene transfer events serve as useful phylogenetic markers $(17,18)$, especially when recipient groups contain wellcalibrated dates. We find that our molecular clock model and root calibration are consistent with time-constrained HGT events requiring that Group II Methanogens predate the cyanobacterial ancestor, and that the halobacterial ancestor predates the Opisthokonta (Table S2). Models with a substantially more recent root age for Archaea would likely violate these constraints.

2.3 Estimates of the Age of the Methanosarcina Ancestor. We estimated the time $\tau_{m}$ of the last common ancestor of known acetoclastic representatives of Methanosarcina by analyzing four independent datasets. Our results, summarized in Table S3, provide the mean $\mu_{i}$ and SD $\sigma i$ of the Gaussian distribution $p\left(\tau_{m} \mid s_{i}\right)$, the probability of $\tau_{m}$ given dataset $s i, i=1, \ldots, 4$. These four independent estimates are then combined into a single probability distribution as follows.

Using Bayes' theorem, we express each independent distribution as

$$
p\left(\tau_{m} \mid s_{i}\right)=\frac{p\left(s_{i} \mid \tau_{m}\right) p\left(\tau_{m}\right)}{p\left(s_{i}\right)}, \quad i=1, \ldots, 4
$$

where $p\left(\tau_{m}\right)$ is the prior distribution for $\tau_{m}$. The probability distribution for $\tau_{m}$ given all four sets of data is

$$
p\left(\tau_{m} \mid s_{1}, \ldots, s_{4}\right)=\frac{p\left(s_{1}, \ldots, s_{4} \mid \tau_{m}\right) p\left(\tau_{m}\right)}{p\left(s_{1}, \ldots, s_{4}\right)}
$$


The independence of the datasets $s i$ implies that their joint distribution factorizes. Consequently,

$$
p\left(\tau_{m} \mid s_{1}, \ldots, s_{4}\right)=p\left(\tau_{m}\right) \prod_{i=1}^{4} \frac{p\left(s_{i} \mid \tau_{m}\right)}{p\left(s_{i}\right)} .
$$

[S38]

Substitution of Eq. S36 into the right-hand side then yields

$$
p\left(\tau_{m} \mid s_{1}, \ldots, s_{4}\right)=p\left(\tau_{m}\right) \prod_{i=1}^{4} \frac{p\left(\tau_{m} \mid s_{i}\right)}{p\left(\tau_{m}\right)} .
$$

We assume that the prior distribution $p\left(\tau_{m}\right)=$ const: Then

$$
p\left(\tau_{m} \mid s_{1}, \ldots, s_{4}\right) \propto \prod_{i=1}^{4} p\left(\tau_{m} \mid s_{i}\right),
$$

The posterior distribution $p(\tau m \mid s 1, \ldots, s 4)$ is therefore the normalized product of the four Gaussian distributions $p\left(\tau_{m} \mid s i\right)$. The resulting distribution is again Gaussian, with mean

$$
\mu=\frac{\sum_{i=1}^{4} \mu_{i} / \sigma_{i}^{2}}{\sum_{i=1}^{4} 1 / \sigma_{i}^{2}}=239.6 \mathrm{Myr}
$$

[S41]

and SD

$$
\sigma=\left(\sum_{i=1}^{4} \frac{1}{\sigma_{i}^{2}}\right)^{-1 / 2}=41.3 \mathrm{Myr} .
$$

2.4 Selection of Relaxed Clock Model. Although all molecular clock studies are subject to model violations and inaccuracies, proper model selection and robust gene sets have both been shown to produce reliable deep-time divergence estimates in the absence of 
fossil calibration data $(16,19)$.

Ref. 23 estimated the age of the Methanosarcina ancestor at about $150 \pm 10$ million years ago (Ma) based on ribosomal RNA sequences, a date substantially more recent than our own estimates. Although this work also included a Bayesian analysis of divergence times within Archaea using a concatenated protein dataset similar to our own, an estimated age based on the protein dataset was not reported for the Methanosarcina ancestor. The ribosomal RNA analysis in ref. 23 makes use of the MultiDivTime program, which implements an autocorrelated Brownian motion relaxed clock model, rather than the uncorrelated gamma multi- pliers relaxed clock model (-ugam) we implement using PhyloBayes. Uncorrelated gamma models outperform autocorrelated models for deep-time phylogenetic analyses, where there is little expectation that evolutionary rates will change smoothly across adjacent branches (24). Furthermore, autocorrelated models are less robust to local violations of rate models, which are tolerated well by uncorrelated models. Uncorrelated models are therefore preferred if the underlying branch rate model is not known (25).

To test the impact of model choice on the age estimate for Methanosarcina, we performed two additional chronogram analyses on ribosomal RNA datasets (16S and 23S) using PhyloBayes, under both uncorrelated gamma and autocorrelated CIR models [a Brownian motion-derived model (26)]. Under the CIR model, $16 S$ and 235 time estimates for Methanosarcina (95 Ma and $173 \mathrm{Ma}$, respectively) were similar to the more recent dates found in ref. 23, whereas, under uncorrelated gamma models, our estimates were $235 \mathrm{Ma}$ and $260 \mathrm{Ma}$, respectively, more consistent with one another and much closer to the ribosomal protein-based age estimates given in Table S3.

2.5 Methanosarcina Strain Growth Conditions. The 16S sequences of four additional species that grow readily on acetate all diverge after the common ancestor of 
acetoclastic Methanosarcina within the ribosomal tree: $M$. sicilae, M. honorobensis, $M$. vacuolata, and M. thermophila (27-32). Four species that are shown not to grow on acetate all diverge before this node: M. semesiae, M. baltica, M. lacustris, and M. sp. HC-2 (28, 3336). To our knowledge, the more deeply branching environmental isolates are not available for additional sequencing. Consequently, growth conditions provide a simple proxy for the presence of $a c k A / p t a$, and are reliable given the well-developed methodologies for characterizing methanogen growth substrates (35). The resulting phylogenetic distribution of growth conditions is simply explained by a singular horizontal gene transfer of ackA/pta within the Methanosarcina genus, immediately preceding the divergence of the acetoclastic groups within our ribosomal tree.

\section{Nickel Analyses}

3.1 Meishan Section and Sampling Methods. A set of drill core and outcrop samples collected from Meishan area, South China, were used for elemental analysis. The core samples are all from the Meishan drill core-1, which spanned the stratigraphic interval from the Late Permian Lungtan Formation to the Early Triassic Yinkeng Formation. A detailed description of the Meishan drilling project undertaken at section A by the Nanjing Institute of Geology and Paleontology in 2004 is included in ref. 37. The outcrop samples were collected from section C, which is in a new quarry $150 \mathrm{~m}$ east of the drill site. The sampling interval spanned $10 \mathrm{~m}$ from the top of the Changxing Formation $(8 \mathrm{~m})$ through the Yinkeng Formation $(2 \mathrm{~m})$, crossing the Permian-Triassic boundary. All rocks from the drill core and outcrop can be correlated lithologically for comparison with the exposed outcrop of section D, which represents the Global Boundary Stratotype Section and Point for the Permian-Triassic boundary and Wuchiapingian- Changhsingian boundary as defined by the conodont zones (37). As a result, the section $C$ outcrop sample heights were converted into the drilling depth for a correlating sequence. 
3.2 Cleaning Procedure. Polyethylene bottles, polypropylene 15-mL tubes, and polypropylene $1.5-\mathrm{mL}$ centrifuge vials were acid- leached with $1 \mathrm{M}$ reagent-grade $\mathrm{HCl}$ for a minimum of $1 \mathrm{~d}$ at $60^{\circ} \mathrm{C}$. The bottles, tubes, and vials were rinsed five times with pure distilled water. After being filled with $0.06 \mathrm{M}$ high purity $\mathrm{HCl}$, the bottles, tubes, and vials were leached for an additional day at $60^{\circ} \mathrm{C}$. Pipette tips were rinsed three times with dilute $\mathrm{HCl}$ and three times with pure distilled water immediately before use. Four $95 \% \mathrm{Pt} / 5 \%$ Au crucibles, Teflon beakers, and Teflon tops were heated in reagent grade $8 \mathrm{M} \mathrm{HNO}_{3}$ for $90 \mathrm{~min}$ at $70^{\circ} \mathrm{C}$ on a hotplate. After rinsing thoroughly with pure distilled water, crucibles, beakers, and tops were air-dried in a laminar-flow clean bench.

3.3 Manual Fusion Procedure. Samples were powdered using a solvent-cleaned ceramic puck mill. Powdered sample material $(\sim 0.100 \mathrm{~g})$ was weighed directly into a preweighed crucible, and then $\sim 0.300 \mathrm{~g}$ of lithium metaborate flux ( $\mathrm{LiBO} 2$; Claisse pure grade) was added to achieve an approximate sample-to-flux ratio of 1:3. Precise masses were recorded. The sample and flux were mixed with an acid-rinsed pipette tip. The crucible containing the sample and flux mixture was heated over a natural gas flame produced by a Meker burner for at least $10 \mathrm{~min}$. Using Pt-tipped tongs, the crucible was gently shaken to agitate the sample and flux mixture. After $10 \mathrm{~min}$, the crucible was plunged into a clean Teflon beaker containing $\sim 15 \mathrm{~mL}$ high purity $1 \mathrm{M}$ HNO3. The Teflon beaker containing the crucible, dilute acid, and sample bead were sonicated for an hour in a deionized water bath. The crucible was removed and rinsed with pure distilled water before being acid leached as described above. The rinsate was collected in the Teflon beaker containing the sample solution before being sonicated for an additional half hour to ensure complete dissolution. The sample solution in the Teflon beaker was decanted into a preweighed, clean 30-mL polypropylene bottle. The Teflon beaker was rinsed with distilled water, and the rinsate was decanted into the same 30$\mathrm{mL}$ polypropylene bottle. The mass of the solution was recorded. The density of the 
sample solution was calculated by weighing $1 \mathrm{~mL}$ of the sample solution using a calibrated pipette. The exact volume of the sample solution in the $30-\mathrm{mL}$ bottle was calculated gravimetrically from the density and the recorded mass. Distilled water (5-10 $\mathrm{mL}$ ) was filtered through a 0.2-m Nalgene syringe filter. Then, $\sim 5 \mathrm{~mL}$ of clean $0.2 \mathrm{M}$ trace metal clean $\mathrm{HNO}_{3}$ followed by $\sim 5 \mathrm{~mL}$ of distilled water were filtered through the filter cartridge and discarded. After filtering and discarding $\sim 2 \mathrm{~mL}$ of sample solution, $\sim 1.5 \mathrm{~mL}$ of sample solution were collected in an acid-cleaned $1.5-\mathrm{mL}$ centrifuge vial. Using an auto pipette, $0.750 \mathrm{~mL}$ of filtered sample solution, $75 \mu \mathrm{L}$ of a $0.2 \mathrm{ppm}$ In solution, and $14.175 \mathrm{~mL}$ of high-purity $0.2 \mathrm{~N} \mathrm{HNO} 3$ were combined in a 15-mL tube for elemental analysis by Quadrupole Inductively Coupled Plasma Mass Spectrometry (ICPMS, Fisons PQ2+). A multielement standard, which included In (1 ppb) and Ni (1 $\mathrm{ppb})$, was analyzed with the samples and used for concentration calculations.

Each sample was independently prepared in duplicate and analyzed in duplicate by ICPMS. Two flux blanks were prepared by weighing $\sim 0.3 \mathrm{~g}$ of $\mathrm{LiBO}_{2}$ and dissolving it in high-purity $1 \mathrm{M} \mathrm{HNO} 3$ acid. The flux blanks for Ni were negligible in comparison with the ICPMS signal for $0.2 \mathrm{M}$ high-purity $\mathrm{HNO}$, which was also low. A rock standard (USGS G-2) was prepared in triplicate, and $6 \mathrm{LiBO}_{2}$ procedural blanks were prepared using the method detailed above. The procedural blanks were all below 1 ppm (expressed as the concentration in $100 \mathrm{mg}$ of sample) with a single exception. The average value for the procedural blanks was $0.7 \mathrm{ppm}$, which was subtracted from the uncorrected G-2 standard concentrations and sample concentrations. The accepted value for the Ni concentration of the G-2 standard is 5:0 $2: 8 \mathrm{ppm}$ (38), which compares well with the average value of $5.5 \mathrm{ppm}$ that we measured for the G-2 standard using the fusion method and ICPMS analysis as described above. The measured bulk Ni concentrations for the duplicates were averaged, and the absolute error was $3.0 \mathrm{ppm}$, on average. Some variability between the duplicates may stem from sample 
inhomogeneities. The measured concentration data for the procedural blanks and G-2 standard concentrations are given in Table S4.

3.4 Carbonate Free Basis Calculation. Lithology is highly variable through the Meishan section. To account for the lithological variability, percent carbonate was measured by decarbonation and a carbonate free basis correction was applied. Approximately $0.5 \mathrm{~g}$ of powdered sample was weighed into a polypropylene vial. Each sample was acidified with $1 \mathrm{~N}$ reagent grade $\mathrm{HCl}$. Multiple treatments were applied until $\mathrm{CO}_{2}$ was no longer evolved. The samples were rinsed with distilled water until $\mathrm{pH}$ neutral was reached. The samples were dried in a $60{ }^{\circ} \mathrm{C}$ oven and reweighed. The fraction $\phi$ of carbonate was calculated based on the difference between the initial and final weights. The carbonatefree- basis concentration, $[\mathrm{Ni}]_{\mathrm{Cfb}}$, was then computed from the bulk concentration $[\mathrm{Ni}]_{\text {bulk }}$ from the formula

$$
[\mathrm{Ni}]_{\mathrm{cfb}}=\frac{[\mathrm{Ni}]_{\text {bulk }}}{1-\phi} \text {. }
$$

This calculation assumes that the $\mathrm{Ni}$ in the carbonate phase is negligible compared with the noncarbonate phase (39). Fig. S3 contains a plot of the carbonate fraction in the section. Table S5 summarizes all analyses. 


\section{References}

1. Rothman DH, Hayes JM, Summons RE (2003) Dynamics of the Neoproterozoic carbon cycle. Proc Natl Acad Sci USA 100(14):8124-8129.

2. Hayes JM, Strauss H, Kaufman AJ (1999) The abundance of ${ }^{13} \mathrm{C}$ in marine organic matter and isotopic fractionation in the global biogeochemical cycle of carbon during the past 800 Ma. Chem Geol 161(1-3):103-125.

3. Des Marais DJ (1997) Long-term evolution of the biogeochemical carbon cycle. Geomicrobiology: Interactions Between Microbes and Minerals, eds Banfield JF, Nealson KH (Mineralogical Society of America, Washington, DC), pp 429-448.

4. Fournier GP, Gogarten JP (2010) Rooting the ribosomal tree of life. Mol Biol Evol 27(8):1792-1801.

5. Williams D, et al. (2011) A rooted net of life. Biol Direct 6:45.

6. Benson DA, et al. (2013) GenBank. Nucleic Acids Res 41(Database issue):D36-D42.

7. Edgar RC (2004) MUSCLE: Multiple sequence alignment with high accuracy and high throughput. Nucleic Acids Res 32(5):1792-1797.

8. Guindon S, et al. (2010) New algorithms and methods to estimate maximum- likelihood phylogenies: Assessing the performance of PhyML 3.0. Syst Biol 59(3): 307-321.

9. Lartillot N, Philippe H (2004) A Bayesian mixture model for across-site heterogeneities in the amino-acid replacement process. Mol Biol Evol 21(6):1095-1109.

10. Venditti C, Meade A, Pagel M (2008) Phylogenetic mixture models can reduce nodedensity artifacts. Syst Biol 57(2):286-293.

11. Feng DF, Cho G, Doolittle RF (1997) Determining divergence times with a protein clock: Update and reevaluation. Proc Natl Acad Sci USA 94(24):13028-13033.

12. Sheridan PP, Freeman KH, Brenchley JE (2003) Estimated minimal divergence times of the major bacterial and archaeal phyla. Geomicrobiol J 20(1):1-14.

13. Martin W, Russell MJ (2003) On the origins of cells: A hypothesis for the evolutionary transitions from abiotic geochemistry to chemoautotrophic prokaryotes, and from prokaryotes to nucleated cells. Philos Trans R Soc Lond B Biol Sci 358(1429):59-83, and discussion (2003) 358:83-85.

14. Boussau B, Blanquart S, Necsulea A, Lartillot N, Gouy M (2008) Parallel adaptations to high temperatures in the Archaean eon. Nature 456(7224):942-945.

15. Gogarten-Boekels M, Hilario E, Gogarten JP (1995) The effects of heavy meteorite bombardment on the early evolution-The emergence of the three domains of life. Orig Life Evol Biosph 25(1-3):251-264. 
16. Peterson KJ, Butterfield NJ (2005) Origin of the Eumetazoa: Testing ecological predictions of molecular clocks against the Proterozoic fossil record. Proc Natl Acad Sci USA 102(27):9547-9552.

17. Szöllosi GJ, Boussau B, Abby SS, Tannier E, Daubin V (2012) Phylogenetic modeling of lateral gene transfer reconstructs the pattern and relative timing of speciations. Proc Natl Acad Sci USA 109(43):17513-17518.

18. Huang J, Gogarten JP (2009) Ancient gene transfer as a tool in phylogenetic reconstruction. Horizontal Gene Transfer, eds Gogarten MB, Gogarten JP, Olendzenski LC (Springer, New York), pp 127-139.

19. Peterson KJ, Cotton JA, Gehling JG, Pisani D (2008) The Ediacaran emergence of bilaterians: Congruence between the genetic and the geological fossil records. Philos Trans R Soc Lond B Biol Sci 363(1496):1435-1443.

20. Huang J, Xu Y, Gogarten JP (2005) The presence of a haloarchaeal type tyrosyl- tRNA synthetase marks the opisthokonts as monophyletic. Mol Biol Evol 22(11): 2142-2146.

21. Schirrmeister BE, de Vos JM, Antonelli A, Bagheri HC (2013) Evolution of multicellularity coincided with increased diversification of cyanobacteria and the Great Oxidation Event. Proc Natl Acad Sci USA 110(5):1791-1796.

22. Cobbe N, Heck MMS (2004) The evolution of SMC proteins: Phylogenetic analysis and structural implications. Mol Biol Evol 21(2):332-347.

23. Blank CE (2009) Not so old Archaea - the antiquity of biogeochemical processes in the archaeal domain of life. Geobiology 7(5):495-514.

24. Drummond AJ, Suchard MA (2010) Bayesian random local clocks, or one rate to rule them all. BMC Biol 8:114.

25. Battistuzzi FU, Filipski A, Hedges SB, Kumar S (2010) Performance of relaxed-clock methods in estimating evolutionary divergence times and their credibility intervals. Mol Biol Evol 27(6):1289-1300.

26. Lepage T, Bryant D, Philippe H, Lartillot N (2007) A general comparison of relaxed molecular clock models. Mol Biol Evol 24(12):2669-2680.

27. Zhilina TN, Zavarzin GA (1987) Methanosarcina vacuolata sp. nov., a vacuolated Methanosarcina. Int J Syst Bacteriol 37(3):281-283.

28. Shimizu S, Upadhye R, Ishijima Y, Naganuma T (2011) Methanosarcina horonobensis sp. nov., a methanogenic archaeon isolated from a deep subsurface Miocene formation. Int J Syst Evol Microbiol 61(Pt 10):2503-2507.

29. Elberson MA, Sowers KR (1997) Isolation of an aceticlastic strain of Methanosarcina 
siciliae from marine canyon sediments and emendation of the species description for Methanosarcina siciliae. Int J Syst Bacteriol 47(4):1258-1261.

30. Terlesky KC, Nelson MJ, Ferry JG (1986) Isolation of an enzyme complex with carbon monoxide dehydrogenase activity containing corrinoid and nickel from acetate- grown Methanosarcina thermophila. J Bacteriol 168(3):1053-1058.

31. Aceti DJ, Ferry JG (1988) Purification and characterization of acetate kinase from acetategrown Methanosarcina thermophila. Evidence for regulation of synthesis. J Biol Chem 263(30):15444-15448.

32. Lundie LL, Jr., Ferry JG (1989) Activation of acetate by Methanosarcina thermophila. Purification and characterization of phosphotransacetylase. J Biol Chem 264(31):1839218396.

33. Lyimo TJ, Pol A, Op den Camp HJ, Harhangi HR, Vogels GD (2000) Methanosarcina semesiae sp. nov., a dimethylsulfide-utilizing methanogen from mangrove sediment.

34. Simankova MV, et al. (2001) Methanosarcina lacustris sp. nov., a new psychrotolerant methanogenic archaeon from anoxic lake sediments. Syst Appl Microbiol 24(3):362-367.

35. Singh N, Kendall MM, Liu Y, Boone DR (2005) Isolation and characterization of methylotrophic methanogens from anoxic marine sediments in Skan Bay, Alaska: Description of Methanococcoides alaskense sp. nov., and emended description of Methanosarcina baltica. Int J Syst Evol Microbiol 55(Pt 6):2531-2538.

36. von Klein D, Arab H, Völker H, Thomm M (2002) Methanosarcina baltica, sp. nov., a novel methanogen isolated from the Gotland Deep of the Baltic Sea. Extremophiles 6(2):103-110.

37. Cao C, et al. (2009) Biogeochemical evidence for euxinic oceans and ecological disturbance presaging the end-Permian mass extinction event. Earth Planet Sci Lett 281(3-4):188-201.

38. Gladney ES, Jones EA, Nickell EJ, Roelandts I (1992) 1988 compilation of elemental concentration data for USGS AGV-1, GSP-1 AND G-2. Geostand Newsl 16(2):111-300.

39. Collier R, Edmond J (1984) The trace element geochemistry of marine biogenic particulate matter. Prog Oceanogr 13(2):113-199. 


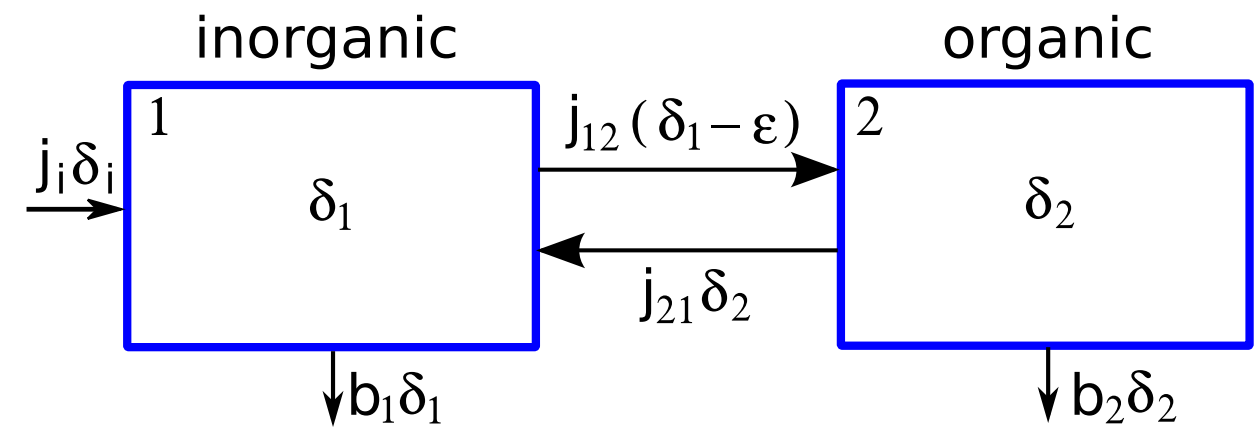

Fig. S1. Isotopic reservoirs and fluxes in a two-compartment idealization of the geochemical carbon cycle (1).
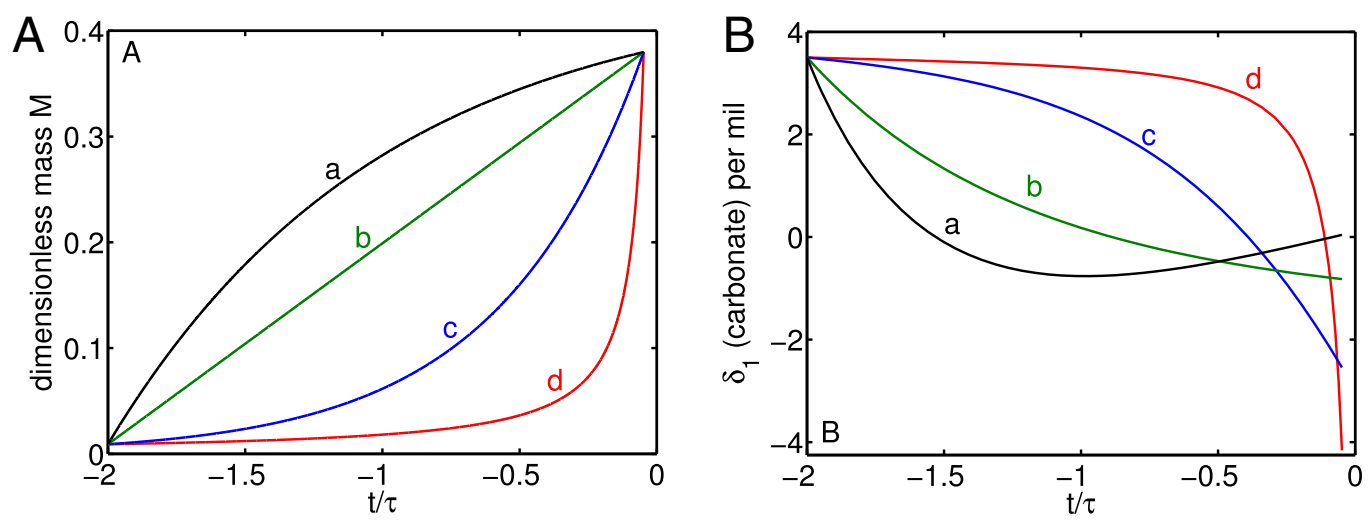

Fig. S2. Examples of sublinear (a), linear (b), exponential (c), and superexponential (d) growth. (A) Curves for the dimensionless mass MðtP. (B) Curves for $\delta 1 ð t \mathrm{t}$.

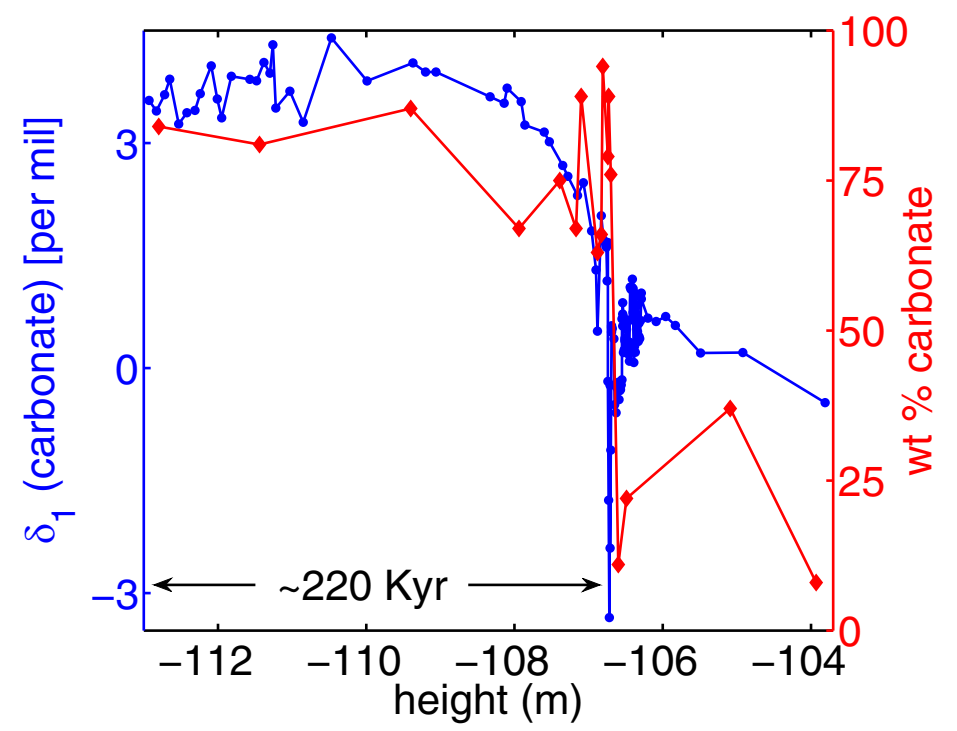

Fig. S3. Plot of the weight percent of carbonate (red) in the Meishan section. The isotopic composition of carbonate (blue) is shown for reference. 
Table S1. Comparison of assumptions related to the analysis of carbon cycles driven out of steady state by changing $\mathrm{ji}$ and by changing $\mathrm{j}_{21}$

\begin{tabular}{lll} 
Quantity & Changing $j_{i}$ & Changing $j_{21}$ \\
\hline$j_{i}$ & varies & constant \\
$j_{12}$ & varies & constant \\
$j_{12}-j_{21}$ & constant & varies \\
$m_{2}$ & constant & varies \\
\hline
\end{tabular}

Table S2. Temporally calibrated HGT events are consistent with estimated divergence times within ribosomal clock model

\begin{tabular}{lcc} 
HGT time constraint & Upper-bound estimate & Lower-bound estimate \\
\hline (donor > recipient) & from clock model $(95 \% \mathrm{Cl})$ & from calibrations \\
Halobacteria > Opisthokonta & $0.55-1.05 \mathrm{Ga}$ & $0.89-0.99 \mathrm{Ga}(19)$ \\
TyrRS protein (20) & & \\
Group II Methanogens* $>$ Cyanobacteria & $2.09-2.7 \mathrm{Ga}$ & $2.3-2.5 \mathrm{Ga}(21)$ \\
SMC protein (22) & &
\end{tabular}

Upper-bound age estimates for the halobacterial ancestor may be underestimated due to low sampling and the unresolved, complex evolutionary history of Halobacteria.

* Maximum-likelihood tree of SMC proteins within Euryarchaeota verified the transfer identified by Cobbe and Heck (22) and confirmed transfer from a donor within the methanogens following the divergence of Archaeoglobales.

Table S3. Estimates of the Methanosarcina ancestor age

\begin{tabular}{lcr} 
Dataset & Mean age, Ma & SD, Ma \\
\hline Universal ribosomal proteins & 227.6 & 54.4 \\
Archaeal ribosomal proteins & 267.9 & 91.4 \\
16S ribosomal RNA & 235.2 & 111.8 \\
23S ribosomal RNA & 259.8 & 141.7 \\
\hline
\end{tabular}


Table S4. Nickel concentrations for blanks and standards

\begin{tabular}{lc} 
Blank and standard replicates & Concentration, ppm \\
\hline Procedural blank A & 0.6 \\
Procedural blank B & 2.6 \\
Procedural blank C & 0.3 \\
Procedural blank D & 0.4 \\
Procedural blank E & 0.2 \\
Procedural blank F & 0.1 \\
Procedural blank average & 0.7 \\
G-2 standard-A & 5.7 \\
G-2 standard-B & 6.1 \\
G-2 standard-C & 4.7 \\
G-2 standard average & 5.5 \\
\hline
\end{tabular}

Table S5. Nickel analyses of the Meishan section

Sample label Height, m Bulk Ni, ppm Ni carbonate free basis, ppm carbonate, $\%$ Ni error, ppm

\begin{tabular}{llrrrr}
\hline MS-1-34-1a & -103.93 & 31.2 & 34.0 & 8 & 1.9 \\
MS-1-32-1a & -105.09 & 19.9 & 31.4 & 37 & 3.5 \\
MS-1-27-1a & -106.49 & 26.7 & 34.2 & 22 & 3.8 \\
MS-1-26-1a & -106.60 & 29.2 & 32.9 & 11 & 3.3 \\
MS24eu & -106.70 & 15.9 & 65.3 & 76 & 2.0 \\
MS24-1a & -106.73 & 26.8 & 248.3 & 89 & 4.7 \\
MS-1-24-8a & -106.74 & 27.2 & 131.8 & 79 & 1.3 \\
MS24-2a & -106.81 & 15.1 & 232.0 & 94 & 2.3 \\
MS24-3a & -106.83 & 10.6 & 30.8 & 66 & 4.6 \\
MS24-4a & -106.88 & 24.6 & 66.1 & 63 & 2.1 \\
MS-1-24-2a & -107.10 & 4.0 & 34.4 & 89 & 0.9 \\
MS24-6a & -107.17 & 28.2 & 84.5 & 67 & - \\
MS24-9a & -107.39 & 25.0 & 100.7 & 75 & 9.4 \\
MS-1-24-6a & -107.94 & 16.7 & 51.2 & 67 & 4.6 \\
MS-1-23-4a & -109.40 & 12.9 & 100.9 & 87 & 1.5 \\
MS05-2b & -111.44 & 15.2 & 78.6 & 81 & 4.7 \\
MS05-4b & -112.80 & 12.1 & 77.5 & 84 & - \\
\hline
\end{tabular}


